\title{
Introduction to Nonequilibrium Quantum Field Theory
}

\author{
Jürgen Berges \\ Institut für Theoretische Physik, Universität Heidelberg \\ Philosophenweg 16, 69120 Heidelberg, Germany \\ http://www. thphys.uni-heidelberg.de/ berges
}

\begin{abstract}
There has been substantial progress in recent years in the quantitative understanding of the nonequilibrium time evolution of quantum fields. Important topical applications, in particular in high energy particle physics and cosmology, involve dynamics of quantum fields far away from the ground state or thermal equilibrium. In these cases, standard approaches based on small deviations from equilibrium, or on a sufficient homogeneity in time underlying kinetic descriptions, are not applicable. A particular challenge is to connect the far-from-equilibrium dynamics at early times with the approach to thermal equilibrium at late times. Understanding the "link" between the earlyand the late-time behavior of quantum fields is crucial for a wide range of phenomena. For the first time questions such as the explosive particle production at the end of the inflationary universe, including the subsequent process of thermalization, can be addressed in quantum field theory from first principles. The progress in this field is based on efficient functional integral techniques, so-called $n$-particle irreducible effective actions, for which powerful nonperturbative approximation schemes are available. Here we give an introduction to these techniques and show how they can be applied in practice. Though we focus on particle physics and cosmology applications, we emphasize that these techniques can be equally applied to other nonequilibrium phenomena in complex many body systems.
\end{abstract}

Based on summer school lectures presented at HADRON-RANP 2004, March 28 - April 3, 2004, Rio de Janeiro, Brazil and at QUANTUM FIELDS IN AND OUT OF EQUILIBRIUM, September $23-27,2003$, Bielefeld, Germany. 


\section{CONTENTS}

1 Motivations and overview 3

1.1 How to describe nonequilibrium quantum fields from first principles? . $\quad 4$

1.1.1 Standard approximation methods fail out of equilibrium _. . . 6

1.1.2 $n$-Particle irreducible expansions: universality and non-secularity 10

$2 n$-Particle irreducible generating functionals I $\quad 12$

2.1 Loop or coupling expansion of the 2PI effective action $\ldots \ldots \ldots$.

2.2 Renormalization . . . . . . . . . . . . . . . . . . . . 17

2.2.1 2PI renormalization scheme to order $\lambda_{R}^{2} \ldots \ldots \ldots \ldots$

2.2.2 Renormalized equations for the two- and four-point functions . 20

2.32 2PI effective action for fermions . . . . . . . . . . . . 22

2.3.1 Chiral quark-meson model . . . . . . . . . . . . 23

2.4 Two-particle irreducible $1 / N$ expansion $\ldots \ldots \ldots \ldots . \ldots . . \ldots 25$

2.4.1 Classification of diagrams . . . . . . . . . . 25

2.4.2 Symmetries and validity of Goldstone's theorem . . . . . . 28

3 Nonequilibrium quantum field theory 29

3.1 Nonequilibrium generating functional . . . . . . . . . . . . . . 29

3.2 Initial conditions . . . . . . . . . . . . . . . . 31

3.3 Nonequilibrium 2PI effective action $\ldots \ldots \ldots \ldots \ldots$

3.4 Exact evolution equations . . . . . . . . . . . . . . . . . 34

3.4.1 Spectral and statistical components . . . . . . . . . . 35

3.4 .2 Detour: Thermal equilibrium . . . . . . . . . . . . . 37

3.4.3 Nonequilibrium evolution equations . . . . . . . . . . 38

3.4 .4 Non-zero field expectation value . . . . . . . . . . . . . . . 42

3.4.5 Lorentz decomposition for fermion dynamics . . . . . . . . . . 43

3.5 Nonequilibrium dynamics from the 2PI loop expansion . . . . . . . . 47

3.6 Nonequilibrium dynamics from the $2 \mathrm{PI} 1 / N$ expansion $\ldots \ldots \ldots$

3.6.1 Nonvanishing field expectation value $\ldots \ldots \ldots . . \ldots 50$

3.7 Numerical implementation . . . . . . . . . . . . . . . . . . 51

4 Nonequilibrium phenomena $\quad 55$

4.1 Scattering, off-shell and memory effects . . . . . . . . . . . 57

4.1 .1 LO fixed points . . . . . . . . . . . . . . 57

4.1.2 NLO thermalization . . . . . . . . . . . . . . . . . 61

4.1.3 Detour: Boltzmann equation . . . . . . . . . . . . . . 65

4.1.4 Characteristic time scales . . . . . . . . . . . . . . . 68

4.2 Prethermalization . . . . . . . . . . . . . . . . 71

4.3 Far-from-equilibrium field dynamics: Parametric resonance . . . . . . 75

4.3.1 Parametric resonance in the $O(N)$ model . . . . . . . . 77

5 Classical aspects of nonequilibrium quantum fields: Precision tests $\quad \mathbf{8 8}$

5.1 Exact classical time-evolution equations . . . . . . . . . . . 89

5.2 Classicality condition . . . . . . . . . . . . . . 92

Introduction to Nonequilibrium Quantum Field Theory 2 
5.3 Precision tests and the role of quantum corrections _ . . . . . . 95

5.3.1 Classical equilibration and quantum thermal equilibrium . . . . 97

$6 \quad n$-Particle irreducible generating functionals II: Equivalence hierarchy $\quad 100$

6.1 Higher effective actions . . . . . . . . . . . . . . . . . . . 102

6.1.1 4PI effective action up to four-loop order corrections . . . . . 104

6.1.2 Equivalence hierarchy for $n \mathrm{PI}$ effective actions $\ldots \ldots \ldots$

6.2 Nonabelian gauge theory with fermions . . . . . . . . . . . 110

6.2.1 Effective action up to four-loop or $\mathscr{O}\left(g^{6}\right)$ corrections $\ldots \ldots .112$

6.2.2 Comparison with Schwinger-Dyson equations . . . . . . . . . 116

6.2.3 Nonequilibrium evolution equations . . . . . . . . . . . 118

6.3 Kinetic theory . . . . . . . . . . . . . . . . . . . . . . . 122

6.3.1 "On-shell" approximations . . . . . . . . . . . . . . 122

6.3 .2 Discussion . . . . . . . . . . . . . . . 127

7 Acknowledgements 128

8 Bibliographical notes $\quad 129$

\section{MOTIVATIONS AND OVERVIEW}

Cosmology is time evolution. Solid theoretical descriptions exist for the temporal and spatial local thermal equilibrium related to the time evolution of a homogeneous and isotropic metric and for small perturbations of this. Furthermore, there are powerful numerical techniques for $\mathrm{N}$-particle simulations. In contrast, research concerning the interplay between fluctuations and the time evolution of fields is still scarce. Their interaction is of crucial importance for the generation of density fluctuations during the inflationary phase of the early universe and of entropy at the end of inflation. The corresponding temperature fluctuations in the cosmic microwave background radiation have led to spectacular high-precision measurements of cosmological parameters. It is the dynamics of fluctuations which decides the question whether the baryon asymmetry was created during a cosmological phase transition. Back reactions of large density fluctuations on the evolution of the cosmic scale factor are also possible.

A frequently employed strategy is to concentrate on classical statistical field theory, which can be simulated numerically. It gives important insights when the number of field quanta per mode is sufficiently large such that quantum fluctuations are suppressed compared to statistical fluctuations. However, classical Rayleigh-Jeans divergences and the lack of genuine quantum effects - such as the approach to quantum thermal equilibrium characterized by Bose-Einstein or Fermi-Dirac statistics - limit their use. A coherent understanding of the time evolution in quantum field theory is required - a program which has made substantial progress in recent years with the development of powerful theoretical techniques. For the first time questions such as the explosive particle production at the end of the inflationary universe, including the subsequent process of thermalization, can be addressed in quantum field theory from first principles. Thermalization leads to the loss of a substantial part of the information about the conditions 
in the early universe. The precise understanding of phenomena out of equilibrium play therefore a crucial role for our knowledge about the primordial universe. Important examples are the density fluctuations, nucleosynthesis or baryogenesis - the latter being responsible for our own existence.

The abundance of experimental data on matter in extreme conditions from relativistic heavy-ion collision experiments, as well as applications in astrophysics and cosmology urge a quantitative understanding of far-from-equilibrium quantum field theory. The initial stages of a heavy-ion collision require considering extreme nonequilibrium dynamics. Connecting this far-from-equilibrium dynamics at early times with the approach to thermal equilibrium at late times is a challenge for theory. The experiments seem to indicate early thermalization whereas the present theoretical understanding of QCD suggests a much longer thermal equilibration time. To resolve these questions, it is important to understand to what "degree" thermalization is required to explain the observations. Different quantities effectively thermalize on different time scales and a complete thermalization of all quantities may not be necessary. For instance, an approximately time-independent equation of state $p=p(\varepsilon)$, characterized by an almost fixed relation between pressure $p$ and energy density $\varepsilon$, may form very early - even though the system is still far from equilibrium! Such prethermalized quantities approximately take on their final thermal values already at a time when the occupation numbers of individual momentum modes still show strong deviations from the late-time Bose-Einstein or Fermi-Dirac distribution. Prethermalization is a universal far-from-equilibrium phenomenon which occurs on time scales dramatically shorter than the thermal equilibration time. In order to establish such a behavior it is crucial to be able to compare between the time scales of prethermalization and thermal equilibration. Approaches based on small deviations from equilibrium, or on a sufficient homogeneity in time underlying kinetic descriptions, are not applicable in this case to describe the required "link" between the early and the late-time behavior.

A successful description of the dynamics of quantum fields away from equilibrium is tightly related to the basic problem of how macroscopic irreversible behavior arises from time-reversal invariant dynamics of quantum fields. This is a fundamental question with most diverse applications. The basic field theoretical techniques, which are required to understand the physics of heavy-ion collision experiments or dynamics in the early universe, are equally relevant for instance for the description of the dynamics of BoseEinstein condensates in the laboratory.

\subsection{How to describe nonequilibrium quantum fields from first principles?}

There are very few ingredients. Nonequilibrium dynamics requires the specification of an initial state at some given time $t_{0}$. This may include a density matrix $\rho_{D}\left(t_{0}\right)$ in a mixed $\left(\operatorname{Tr} \rho_{D}^{2}\left(t_{0}\right)<1\right)$ or pure state $\left(\operatorname{Tr} \rho_{D}^{2}\left(t_{0}\right)=1\right)$. Nonequilibrium means that the initial density matrix does not correspond to a thermal equilibrium density matrix: $\rho_{D}\left(t_{0}\right) \neq \rho_{D}^{(\mathrm{eq})}$ with for instance $\rho_{D}^{(\mathrm{eq})} \sim e^{-\beta H}$ for the case of a canonical thermal ensemble with inverse temperature $\beta$. In contrast to close-to-equilibrium field theory, 
the initial density matrix $\rho_{D}\left(t_{0}\right)$ may deviate substantially from thermal equilibrium. This preempts the use of (non-)linear response theory, which is based on sufficiently small deviations from equilibrium, or assumptions about the validity of a fluctuationdissipation relation. Since time-translation invariance is explicitly broken at initial times, there will be no assumption about a sufficient homogeneity in time underlying effective kinetic descriptions. In their range of applicability these properties should come out of the calculation.

Completely equivalent to the specification of the initial density matrix $\rho_{D}\left(t_{0}\right)$ is the knowledge of all initial correlation functions: the initial one-point function $\operatorname{Tr}\left\{\rho_{D}\left(t_{0}\right) \Phi\left(t_{0}, \mathbf{x}\right)\right\}$, two-point function $\operatorname{Tr}\left\{\rho_{D}\left(t_{0}\right) \Phi\left(t_{0}, \mathbf{x}\right) \Phi\left(t_{0}, \mathbf{y}\right)\right\}$, three-point function etc., where $\Phi(t, \mathbf{x})$ denotes a Heisenberg field operator. Typically, the "experimental setup" requires only knowledge about a few lowest correlation functions at time $t_{0}$, whereas complicated higher correlation functions often build up at later times. The question that nonequilibrium quantum field theory addresses concerns the behavior of these correlation functions for $t>t_{0}$ from which one can extract the time evolution of other quantities such as occupation numbers. This is depicted schematically in the figure below. Of course, one could equally evolve the density matrix in time and compute observable quantities such as correlations from it at some later time. However, this is in general much less efficient than directly expressing the dynamics in terms of correlations, which we will do here (cf. Sec. 6).

Once the nonequilibrium initial state is specified, the time-evolution is completely determined by the Hamiltonian or, equivalently, the dynamics can be described in terms of a functional path integral with the classical action $S$. From the latter one obtains the effective action $\Gamma$, which is the generating functional for all correlation functions of the quantum theory (cf. Sec. 2). There are no further ingredients involved concerning the dynamics than what is known from standard vacuum quantum field theory. Here we consider closed systems without coupling to an external heat bath or external fields. There is no course graining or averaging involved in the dynamics. In this respect, the analysis is very different from irreversible phenomenological approaches. The fact that the dynamics is obtained from an effective action automatically guarantees a number of crucial properties for the time evolution. The most important one is conservation of

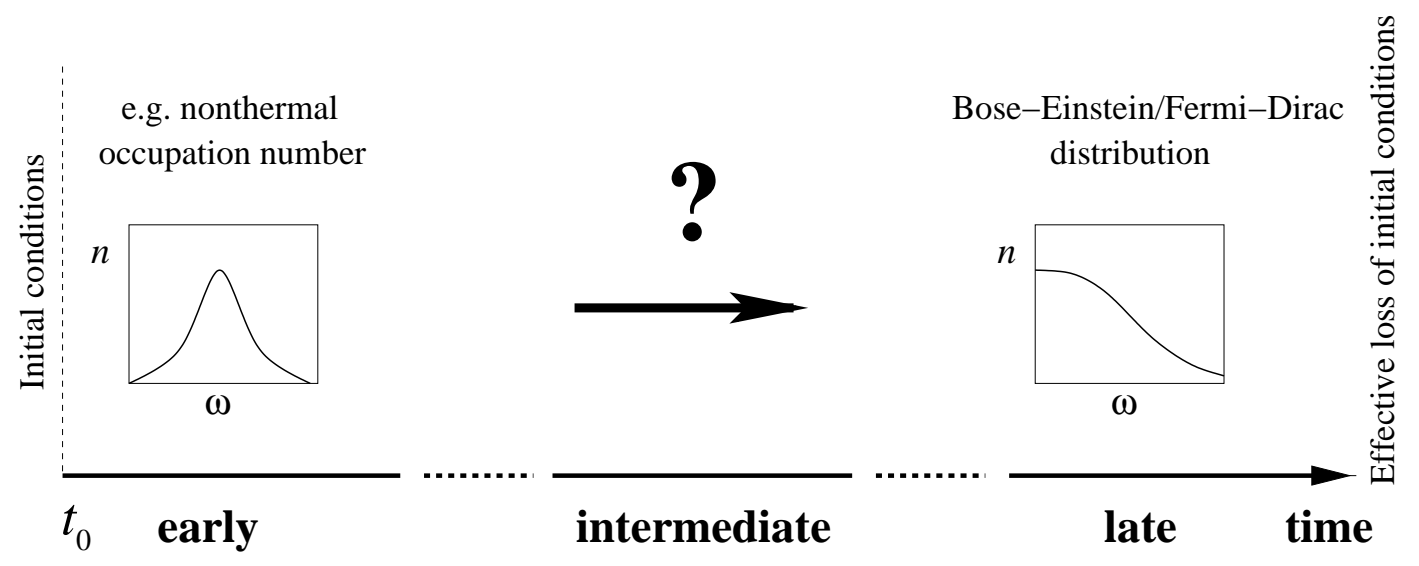


energy. The analogue in classical mechanics is well known: if the equations of motion can be derived from a given action then they will not admit any friction term without further approximations.

It should be stressed that during the nonequilibrium time evolution there is no loss of information in any strict sense. The important process of thermalization is a nontrivial question in a calculation from first principles. Thermal equilibrium keeps no memory about the time history except for the values of a few conserved charges. Equilibrium is time-translation invariant and cannot be reached from a nonequilibrium evolution on a fundamental level. It is striking that we will observe below that the evolution can go very closely towards thermal equilibrium results without ever deviating from them for accessible times. The observed effective loss of details about the initial conditions can mimic very accurately the irreversible dynamics obtained from phenomenological descriptions in their range of applicability.

\subsubsection{Standard approximation methods fail out of equilibrium}

For out-of-equilibrium calculations there are additional complications which do not appear in vacuum or thermal equilibrium. ${ }^{1}$ The first new aspect concerns secularity: The perturbative time evolution suffers from the presence of spurious, so-called secular terms, which grow with time and invalidate the expansion even in the presence of a weak coupling. Here it is important to note that the very same problem appears as well for nonperturbative approximation schemes such as standard $1 / N$ expansions, where $N$ denotes the number of field components. ${ }^{2}$ Typically, secularity is a not a very difficult problem and for a given approximation there can be various ways to resolve it. There is a requirement, however, which poses very strong restrictions on the possible approximations: Universality, i.e. the insensitivity of the late-time behavior to the details of the initial conditions. If thermal equilibrium is approached then the late-time result is universal in the sense that it becomes uniquely determined by the energy density and further conserved charges. To implement the necessary nonlinear dynamics is demanding. Both requirements of a non-secular and universal behavior can indeed be fulfilled using efficient functional integral techniques: so-called n-particle irreducible effective actions, for which powerful nonperturbative approximation schemes are available. They provide a practical means to describe far-from-equilibrium dynamics as well as thermalization from first principles.

An illustrative example from classical mechanics. It is instructive to consider for a moment the simple example of the time evolution of a classical anharmonic oscillator.

\footnotetext{
1 This does not concern restrictions to sufficiently small deviations from thermal equilibrium, such as described in terms of (non-)linear response theory, which only involve thermal equilibrium correlators in real time.

2 Note that restrictions to mean-field type approximations such as leading-order large- $N$ are insufficient. They suffer from the presence of an infinite number of spurious conserved quantities, and are known to fail to describe thermalization. Secularity enters the required next-to-leading order corrections and beyond. (Cf. Sec. 4.)
} 
This exercise will explain some general problems, which one encounters using perturbative techniques for initial-value problems, and indicates how to resolve them. Below we will consider an illustrative "translation" of the outcome to the case of nonequilibrium quantum fields. One of the benefits will be that important concepts such as $n$-particle irreducible effective actions appear here in a very intuitive way, before they will be thoroughly discussed in later sections.

Our damped oscillator with time-dependent amplitude $y(t)$ is characterized by an infinite number of anharmonic terms:

$$
\ddot{y}+y=-\varepsilon \dot{y}-(\varepsilon y)^{3}-(\varepsilon y)^{5}-(\varepsilon y)^{7}-\ldots \quad, \quad \varepsilon \ll 1
$$

Here each dot denotes a derivative with respect to time $t$. The presence of anharmonic terms to arbitrarily high order is reminiscent of the situation in quantum field theory (QFT), where the presence of quantum fluctuations can induce self-interactions to high powers in the field. Consider the ideal case for perturbative estimates, i.e. the presence of a small parameter $\varepsilon$ which suppresses the contributions from higher powers in $y$. The example is chosen such that it can be easily solved without further approximations numerically by summing the geometric series with $\sum_{n=3}^{\infty}(\varepsilon y)^{n}=(\varepsilon y)^{3} /\left(1-\varepsilon^{2} y^{2}\right)$. For the above second-order differential equation the intial-value problem is defined by giving the amplitude and its first derivative at initial time $t=0$. We employ e.g. $y(0)=1$, $\dot{y}(0)=-\varepsilon / 2$ and consider the time evolution for $t \geq 0$. One finds the expected damped oscillator behavior displayed below for $\varepsilon=0.1$ :

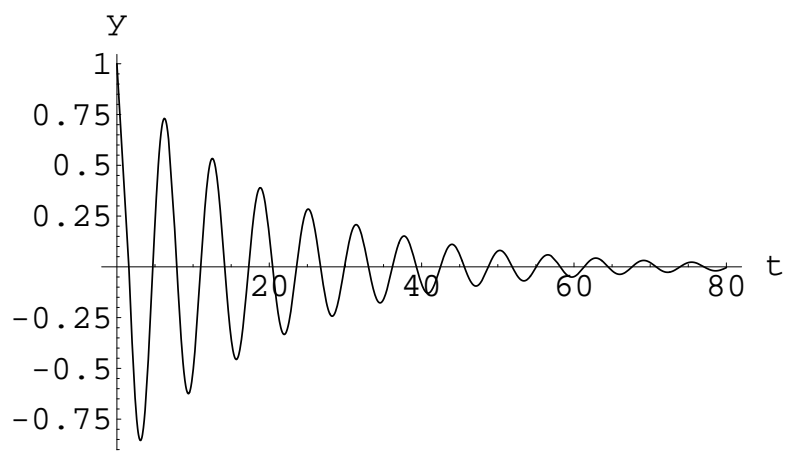

The question is whether an accurate approximate description of the full $y(t)$ is possible if contributions from higher powers of $\varepsilon$ are neglected? In view of the presence of the small expansion parameter this is certainly possible, however, standard perturbation theory fails dramatically to provide a good description. A perturbative expansion of $y$ in $\varepsilon$,

$$
y_{\text {pert }}(t)=y_{0}(t)+\varepsilon y_{1}(t)+\frac{\varepsilon^{2}}{2} y_{2}(t)+\mathscr{O}\left(\varepsilon^{3}\right),
$$

yields the standard hierarchy of equations:

$$
\ddot{y}_{0}(t)+y_{0}(t)=0 \quad, \quad \ddot{y}_{1}(t)+y_{1}(t)=-\dot{y}_{0} \quad, \quad \ldots
$$

These can be iteratively solved as

$$
y_{0}(t)=\frac{1}{2} e^{i t}+\text { c.c. } \quad, \quad y_{1}(t)=-\frac{1}{4} e^{i t} t+\text { c.c. } \quad, \quad \ldots
$$


Doing this to second order in $\varepsilon$ one finds:

$$
y_{\text {pert }}(t)=\frac{1}{2} e^{i t}\left(1-\frac{\varepsilon}{2} t+\frac{\varepsilon^{2}}{8}\left[t^{2}-i t\right]\right)+\text { c.c. }
$$

Beyond the lowest order, the solution contains secular terms which grow with powers of the time $t$ and one arrives at the important conclusion:

$$
\text { The perturbative expansion is only valid for } \varepsilon t \ll 1
$$

We now consider an alternative ("nPI type") expansion: For this we classify the terms of the equation of motion (1) according to powers of the small parameter $\varepsilon$. The lowest order takes into account all terms of the equation to order $\varepsilon^{0}$, which to this order gives the same as in perturbation theory:

$$
\ddot{y}_{n \mathrm{PI}}^{(0)}+y_{n \mathrm{PI}}^{(0)}=0 \Rightarrow y_{n \mathrm{PI}}^{(0)}(t)=\frac{1}{2} e^{i t}+\text { c.c. }
$$

The next order takes into account all terms of (1) to order $\varepsilon$ :

$$
\ddot{y}_{n \mathrm{PI}}^{(2)}+y_{n \mathrm{PI}}^{(2)}=-\varepsilon \dot{y}_{n \mathrm{PI}}^{(2)} \Rightarrow y_{n \mathrm{PI}}^{(2)}(t)=\frac{1}{2} e^{i t \sqrt{1-\varepsilon^{2} / 4}-\varepsilon t / 2}+\text { c.c. }
$$

Since there are no $\varepsilon^{2}$ terms appearing in (1), this also agrees with the second order of the expansion. The next order would take into account all terms of the equation to order $\varepsilon^{3}$ and so on. This provides a systematic approximation procedure in terms of powers of the small parameter $\varepsilon$. However, in contrast to the perturbative expansion the " $n \mathrm{PI}$ type" expansion turns out to be non-secular in time. One explicitly observes that both the lowest order approximation and $y_{n \mathrm{PI}}^{(2)}(t)$ do not exceed $\mathscr{O}\left(\varepsilon^{0}\right)$ for all times. Therefore, the result (7) provides an approximation to the equation (1) up to quantitative corrections of order $\varepsilon^{3}$ at all times. The already very good agreement with the exact result can be checked explicitly.

It is instructive to compare the " $n$ PI type" solution to the perturbative one by expanding (7) in powers of $\varepsilon$ :

$$
\begin{aligned}
y_{n \mathrm{PI}}^{(2)}(t) & =\frac{1}{2} e^{i t \sqrt{1-\varepsilon^{2} / 4}-\varepsilon t / 2}+\text { c.c. } \\
& =\frac{1}{2} e^{i t}\left(1-\frac{\varepsilon}{2} t+\frac{\varepsilon^{2}}{8}\left[t^{2}-i t\right]\right)+\mathscr{O}\left(\varepsilon^{3}\right)+\text { c.c. } \\
& \stackrel{(!)}{=} y_{\text {pert }}(t)+\mathscr{O}\left(\varepsilon^{3}\right)+\text { c.c. }
\end{aligned}
$$

We conclude that

- the " $n \mathrm{PI}$ " result corresponds to the perturbative one up to the order of approximation (here up to $\mathscr{O}\left(\varepsilon^{3}\right)$ corrections).

- the " $n$ PI" result resums all secular terms to infinite perturbative order in $\varepsilon$. 
Stated differently: infinite summation of perturbative orders is required to obtain a uniform approximation to the exact solution, i.e. that the error stays of a given order at all times.

What was the reason for the "success" of this alternative expansion? It is based on a standard procedure for differential equations in order to enlarge the convergence radius of an expansion. In the differential equation one neglects contributions from higher powers of the expansion parameter. However, in contrast to perturbation theory one does not expand in addition the variable $y$. This procedure is sometimes called "selfconsistent" since at each order in the approximation the dynamics is solely expressed in terms of the dynamical degree of freedom itself. For instance, in Eq. (7) both on the left and on the right hand side of the differential equation appear derivatives of the same variable $y_{n \mathrm{PI}}^{(2)}$. In contrast, in the perturbative hierarchy (3) the presence of "external" oscillating "source terms" such as $y_{0}$ driving $y_{1}$ leads to secular behavior. The procedure of the " $n$ PI type" expansion is simple and turns out to be very efficient to achieve non-secular behavior: For given dynamical degrees of freedom truncate the dynamics according to powers of a small expansion parameter. Of course, the choice of the degrees of freedom is always based on physics. The price to be paid for this expansion scheme is that at some order one necessarily has to solve nonlinear equations without further approximations. However, as we will see next, it is precisely the nonlinearity which is required to be able to obtain universality in the sense mentioned above.

In order to illustrate properly the aspect of universality the single oscillator example (1) is too simple. In order to include more degrees of freedom we add a "threemomentum" label to the variables and consider:

$$
\dot{y}_{\mathbf{p}} \sim \int_{\mathbf{q} \mathbf{k}}\left[\left(1+y_{\mathbf{p}}\right)\left(1+y_{\mathbf{q}}\right) y_{\mathbf{k}} y_{\mathbf{p}-\mathbf{q}-\mathbf{k}}-y_{\mathbf{p}} y_{\mathbf{q}}\left(1+y_{\mathbf{k}}\right)\left(1+y_{\mathbf{p}-\mathbf{q}-\mathbf{k}}\right)\right] .
$$

Here the integrals on the r.h.s. involve momenta $\mathbf{q}$ and $\mathbf{k}$ above some suitable value, and one observes that the time derivative of $y_{\mathbf{p}}$ is proportional to a "gain" and a "loss" term. This is like in a Boltzmann equation, which describes the rate of scattering of particles into the state with momentum $\mathbf{p}$ minus the rate of scattering out of that momentum (cf. Sec. 4.1.3). If the nonlinear equation (8) is solved without further approximations then the late-time solution is given by the well-known result

$$
y_{\mathbf{p}} \rightarrow \frac{1}{e^{\beta(|\mathbf{p}|-\mu)}-1},
$$

with real parameters $\beta$ and $\mu$, which for the Boltzmann equation are identified as temperature and chemical potential respectively. The form of the solution (9) is universal, i.e. completely independent of the details of the initial condition for the solution of (8). We emphasize that the Boltzmann type equation (8) is "self-consistent" in the sense mentioned above. In contrast, a non-"self-consistent" approximation will not show the desired universality in general. For instance, consider (8) in a linearized approximation

$$
\dot{y}_{\mathbf{p}}=\left(1+y_{\mathbf{p}}\right) \sigma_{\mathbf{p}}^{0}-y_{\mathbf{p}}{\overline{\sigma_{\mathbf{p}}}}^{0},
$$


with time-independent $\sigma_{\mathbf{p}}^{0} \sim \int_{\mathbf{q k}}\left(1+y_{\mathbf{q}}(0)\right) y_{\mathbf{k}}(0) y_{\mathbf{p}-\mathbf{q}-\mathbf{k}}(0)$ and equivalently for $\bar{\sigma}_{\mathbf{p}}^{0}$. Eq. (10) has the solution

$$
y_{\mathbf{p}}=\frac{\sigma_{\mathbf{p}}^{0}}{\gamma_{\mathbf{p}}^{0}}+\left[y_{\mathbf{p}}(0)-\frac{\sigma_{\mathbf{p}}^{0}}{\gamma_{\mathbf{p}}^{0}}\right] e^{-\gamma_{\mathbf{p}}^{0} t},
$$

with $\gamma_{\mathbf{p}}^{0}=\sigma_{\mathbf{p}}^{0}-\bar{\sigma}_{\mathbf{p}}^{0}$. For late times $\gamma_{\mathbf{p}}^{0} t \gg 1$ this always depends on the chosen initial $\sigma_{\mathbf{p}}^{0} / \gamma_{\mathbf{p}}^{0}$ and is, of course, only useful if they are chosen to be already the equilibrium values.

\subsection{2. n-Particle irreducible expansions: universality and non-secularity}

One should not misunderstand the following illustrative "translation" of the above mechanics examples to QFT. It is intended to give intuitive insight. The questions of secularity and universality are of course settled by actual nonequilibrium calculations in QFT, which is the topic of the main body of this text. For a moment, let us transcribe the above results to the language of QFT. The amplitude $y$ of the above oscillator example plays the role of the one-point function, i.e. the field expectation value or macroscopic field. The amplitude squared plays the role of the two-point function or propagator, the cubic amplitude that of the three-point function or proper three-vertex, etc.:

$$
\begin{aligned}
& y(t) \quad " \rightarrow " \quad \phi(x)=\langle\hat{\Phi}(x)\rangle \\
& \text { (macroscopic field) } \\
& y^{2}(t) \quad " \rightarrow " \quad G_{2}(x, y)=\langle T \hat{\Phi}(x) \hat{\Phi}(y)\rangle-\phi(x) \phi(y) \equiv G(x, y) \\
& \text { (propagator) } \\
& y^{3}(t) \quad " \rightarrow " \quad G_{3}(x, y, z) \text { or, with } G_{3} \sim G G G V_{3}: \\
& V_{3}(x, y, z) \quad \text { (proper three-vertex) } \\
& y^{4}(t) \quad " \rightarrow \cdots \quad V_{4}(x, y, z, w) \quad \text { (proper 4-vertex) }
\end{aligned}
$$

where the symbol $T$ denotes time-ordering. The knowledge of all $n$-point functions provides a full description of the quantum theory. In contrast to the classical example, the information contained e.g. in the two-point function cannot be recovered from the one-point function: $G \not \phi^{2}$ etc. In QFT all $n$-point functions $\phi, G, V_{3}, V_{4} \ldots$ constitute the set of dynamical "degrees of freedom".

The equations of motions for all $n$-point functions are conveniently encoded in a generating functional or effective action. There are different functional representations of the effective action. The so-called n-particle irreducible (nPI) effective action $\Gamma\left[\phi, G, V_{3}, \ldots, V_{n}\right]$ is expressed in terms of $\phi, G, V_{3}, \ldots, V_{n}$ and is particularly efficient for a description of suitable approximation schemes in nonequilibrium quantum field theory. It determines the equations of motion for $\phi, G, V_{3}, \ldots, V_{n}$ by first-order functional 
derivatives or so-called stationarity conditions:

$$
\frac{\delta \Gamma\left[\phi, G, V_{3}, \ldots\right]}{\delta \phi}=0, \frac{\delta \Gamma\left[\phi, G, V_{3}, \ldots\right]}{\delta G}=0, \frac{\delta \Gamma\left[\phi, G, V_{3}, \ldots\right]}{\delta V_{3}}=0, \ldots
$$

The above " $n$ PI" approximation scheme for the oscillator example reads now:

- Classify the contributions to $\Gamma\left[\phi, G, V_{3}, \ldots\right]$ according to powers of a suitable expansion parameter (e.g. coupling/loops, or $1 / N$ ). In terms of the "variables" $\phi, G$, $V_{3} \ldots$ there is a definite answer for the approximate effective action to a given order of the expansion.

- The equations of motion for $\phi, G, V_{3} \ldots$ are then determined from the approximate effective action according to (12) without any further assumptions.

In general this leads to nonlinear integro-differential equations, which typically require numerical solution techniques if one wants to provide the "link" between the early and the late-time behavior. We emphasize that nonlinearity is a crucial ingredient for latetime universality. The reward is that one can compute far-from-equilibrium dynamics as well as the subsequent approach to thermal equilibrium in quantum field theory from first principles (cf. Sec. 4). In turn this can be used to derive powerful effective descriptions and to determine their range of applicability.

In order to be useful in practice it is crucial to observe that it is not necessary to calculate the most general $\Gamma\left[\phi, G, V_{3}, \ldots, V_{n}\right]$ for arbitrarily large $n$. There exists an equivalence hierarchy between $n \mathrm{PI}$ effective actions. For instance, in the context of a loop expansion one has:

$$
\begin{aligned}
\Gamma^{(1 \text { loop })}[\phi] & =\Gamma^{(1 \text { loop })}[\phi, D]=\ldots, \\
\Gamma^{(2 \text { loop })}[\phi] & \neq \Gamma^{(2 \text { loop })}[\phi, D]=\Gamma^{(2 \text { loop })}\left[\phi, D, V_{3}\right]=\ldots, \\
\Gamma^{(3 \text { loop })}[\phi] & \neq \Gamma^{(3 \text { loop })}[\phi, D] \neq \Gamma^{(3 \text { loop })}\left[\phi, D, V_{3}\right]=\Gamma^{(3 \text { loop })}\left[\phi, D, V_{3}, V_{4}\right]=\ldots,
\end{aligned}
$$

where $\Gamma^{(n-\text { loop })}$ denotes the approximation of the respective effective action to $n$-th loop order in the absence of external sources. E.g. for a two-loop approximation all $n$ PI descriptions with $n \geq 2$ are equivalent and the 2PI effective action captures already the complete answer for the self-consistent description up to this order. In contrast, a self-consistently complete result to three-loop order requires at least the 3PI effective action in general, etc. There are, however, other simplifications which can decrease the hierarchy even further such that lower $n \mathrm{PI}$ effective actions are often sufficient in practice. For instance, for a vanishing macroscopic field one has $\Gamma_{2 \mathrm{PI}}^{(31 \text { oop })}[\phi=0, G]=$ $\Gamma_{4 \mathrm{PI}}^{(3 \text { loop })}\left[\phi=0, G, V_{3}=0, V_{4}\right]=\ldots$ in the absence of sources. Typically the 2PI, 3PI or maybe the 4PI effective action captures already the complete answer for the selfconsistent description to the desired/computationally feasible order of approximation. 


\section{2. $N$-PARTICLE IRREDUCIBLE GENERATING FUNCTIONALS I}

In this section we discuss the construction of the one-particle irreducible (1PI) and the two-particle irreducible (2PI) effective action. The latter provides a powerful starting point for systematic approximations in nonequilibrium quantum field theory. To be specific, we consider first a quantum field theory for a real, $N$-component scalar field $\varphi_{a}$ $(a=1, \ldots, N)$ with classical action $S[\varphi]:^{3}$

$$
S[\varphi]=\int_{x}\left(\frac{1}{2} \partial^{\mu} \varphi_{a}(x) \partial_{\mu} \varphi_{a}(x)-\frac{m^{2}}{2} \varphi_{a}(x) \varphi_{a}(x)-\frac{\lambda}{4 ! N}\left(\varphi_{a}(x) \varphi_{a}(x)\right)^{2}\right) .
$$

Here summation over repeated indices is implied and we use the shorthand notation $\int_{x} \equiv \int_{\mathscr{C}} \mathrm{d} x^{0} \int \mathrm{d}^{d} x$ with $x \equiv\left(x^{0}, \mathbf{x}\right)$. The time path $\mathscr{C}$ will be specified later.

The generating functional $W[J, R]$ for connected Green's functions in the presence of two source terms $\sim J_{a}(x)$ and $\sim R_{a b}(x, y)$ is given by

$$
\begin{aligned}
Z[J, R] & =\exp (i W[J, R]) \\
& =\int \mathscr{D} \varphi \exp \left(i\left[S[\varphi]+\int_{x} J_{a}(x) \varphi_{a}(x)+\frac{1}{2} \int_{x y} R_{a b}(x, y) \varphi_{a}(x) \varphi_{b}(y)\right]\right) .
\end{aligned}
$$

We define the macroscopic field $\phi_{a}$ and the connected two-point function $G_{a b}$ by variation of $W$ in the presence of the source terms:

$$
\frac{\delta W[J, R]}{\delta J_{a}(x)}=\phi_{a}(x) \quad, \quad \frac{\delta W[J, R]}{\delta R_{a b}(x, y)}=\frac{1}{2}\left(\phi_{a}(x) \phi_{b}(y)+G_{a b}(x, y)\right) .
$$

Before constructing the 2PI effective action, we consider first the 1PI effective action. It is obtained by a Legendre transform with respect to the source term which is linear in the field,

$$
\Gamma^{R}[\phi]=W[J, R]-\int_{x} \frac{\delta W[J, R]}{\delta J_{a}(x)} J_{a}(x)=W[J, R]-\int_{x} \phi_{a}(x) J_{a}(x) .
$$

We note that:

1. $\Gamma^{R \equiv 0}[\phi]$ corresponds to the standard 1PI effective action.

2. For $R \neq 0$ it can be formally viewed as the 1PI effective action for a theory governed by the modified classical action, $S^{R}[\varphi]$, in the presence of a non-constant "mass term" $\sim R_{a b}(x, y)$ quadratic in the fields:

$$
S^{R}[\varphi]=S[\varphi]+\frac{1}{2} \int_{x y} R_{a b}(x, y) \varphi_{a}(x) \varphi_{b}(y) .
$$

This can be directly observed from (14) and the fact that the standard 1PI effective action is obtained from the same defining functional integral in the presence of a linear source

\footnotetext{
${ }^{3}$ We always work in units where $\hbar=c=1$ such that the mass of a particle is equal to its rest energy $\left(m c^{2}\right)$ and also to its inverse Compton wavelength $(m c / \hbar)$.
} 
term only. As a consequence, it is straightforward to recover for $\Gamma^{R}[\phi]$ all "textbook" relations for the 1PI effective action taking into account $R$. For instance, $\Gamma^{R}[\phi]$ to oneloop order is given by

$$
\Gamma^{R(1 \text { loop })}[\phi]=S^{R}[\phi]+\frac{i}{2} \operatorname{Tr} \ln \left[G_{0}^{-1}(\phi)-i R\right]
$$

which is the familiar result for the 1PI effective action with $S[\phi] \rightarrow S^{R}[\phi]$ and $G_{0}^{-1}(\phi) \rightarrow$ $G_{0}^{-1}(\phi)-i R$. Similarly, one finds that the exact inverse propagator is obtained by second functional field differentiation,

$$
\begin{aligned}
\frac{\delta^{2} \Gamma^{R}[\phi]}{\delta \phi_{a}(x) \delta \phi_{b}(y)} & =i G_{a b}^{-1}(x, y) \\
& =i\left[G_{0, a b}^{-1}(x, y)-i R_{a b}(x, y)-\Sigma_{a b}^{R}(x, y)\right]
\end{aligned}
$$

Here the classical inverse propagator $i G_{0, a b}^{-1}(x, y ; \phi)=\delta^{2} S[\phi] / \delta \phi_{a}(x) \delta \phi_{b}(y)$ reads

$$
\begin{aligned}
i G_{0, a b}^{-1}(x, y ; \phi)= & -\left(\square_{x}+m^{2}+\frac{\lambda}{6 N} \phi_{c}(x) \phi_{c}(x)\right) \delta_{a b} \delta(x-y) \\
& -\frac{\lambda}{3 N} \phi_{a}(x) \phi_{b}(x) \delta(x-y),
\end{aligned}
$$

and $\Sigma_{a b}^{R}(x, y)$ is the proper self-energy, to which only one-particle irreducible Feynman diagrams contribute, i.e. diagrams which cannot be separated by opening one line. These relations will be used below.

We now perform a further Legendre transform of $\Gamma^{R}[\phi]$ with respect to the source $R$ in order to arrive at the 2PI effective action:

$$
\begin{aligned}
\Gamma[\phi, G] & =\Gamma^{R}[\phi]-\int_{x y} \underbrace{\frac{\delta W, R]}{\delta R_{a b}(x, y)}}_{\frac{\delta \Gamma^{R}[\phi]}{\delta R_{a b}(x, y)}}=\frac{1}{2}\left(\phi_{a}(x) \phi_{b}(y)+G_{a b}(x, y)\right) \\
& =\Gamma^{R}[\phi]-\frac{1}{2} \int_{x y} R_{a b}(x, y) \phi_{a}(x) \phi_{b}(y)-\frac{1}{2} \operatorname{Tr} R G .
\end{aligned}
$$

Here we have used that the relation between $\phi$ and $J$ is $R$-dependent, i.e. inverting $\phi=\delta W[J, R] / \delta J$ yields $J=J^{R}(\phi)$. From the above definition of $\Gamma^{R}[\phi]$ one therefore finds

$$
\begin{aligned}
\frac{\delta \Gamma^{R}[\phi]}{\delta R_{a b}(x, y)} & =\frac{\delta W[J, R]}{\delta R_{a b}(x, y)}+\int_{z} \frac{\delta W[J, R]}{\delta J_{c}(z)} \frac{\delta J_{c}(z)}{\delta R_{a b}(x, y)}-\int_{z} \phi_{c}(z) \frac{\delta J_{c}(z)}{\delta R_{a b}(x, y)} \\
& =\frac{\delta W[J, R]}{\delta R_{a b}(x, y)}
\end{aligned}
$$


Of course, the two subsequent Legendre transforms which have been used to arrive at (21) agree with a simultaneous Legendre transform of $W[J, R]$ with respect to both source terms:

$$
\begin{aligned}
\Gamma[\phi, G] & =W[J, R]-\int_{x} \frac{\delta W[J, R]}{\delta J_{a}(x)} J_{a}(x)-\int_{x y} \frac{\delta W[J, R]}{\delta R_{a b}(x, y)} R_{a b}(x, y) \\
& =W[J, R]-\int_{x} \phi_{a}(x) J_{a}(x)-\frac{1}{2} \int_{x y} R_{a b}(x, y) \phi_{a}(x) \phi_{b}(y)-\frac{1}{2} \operatorname{Tr} G R
\end{aligned}
$$

From this one directly observes the stationarity conditions:

$$
\begin{aligned}
\frac{\delta \Gamma[\phi, G]}{\delta \phi_{a}(x)} & =-J_{a}(x)-\int_{y} R_{a b}(x, y) \phi_{b}(y), \\
\frac{\delta \Gamma[\phi, G]}{\delta G_{a b}(x, y)} & =-\frac{1}{2} R_{a b}(x, y),
\end{aligned}
$$

which give the equations of motion for $\phi$ and $G$ in the absence of the sources, i.e. $J=0$ and $R=0$.

To get familiar with Eq. (21) or (23), we may directly calculate $\Gamma[\phi, G]$ to one-loop order using the above results for $\Gamma^{R}[\phi]$. Plugging (18) into (21) one finds to this order

$$
\Gamma[\phi, G] \simeq S[\phi]+\frac{i}{2} \operatorname{Tr} \ln \left[G_{0}^{-1}(\phi)-i R\right]-\frac{1}{2} \operatorname{Tr} R G .
$$

If we set $G^{-1}=G_{0}^{-1}(\phi)-i R$ then we can write

$$
\Gamma[\phi, G] \simeq S[\phi]+\frac{i}{2} \operatorname{Tr} \ln G^{-1}+\frac{i}{2} \operatorname{Tr} G_{0}^{-1} G+\text { const },
$$

with $\operatorname{Tr} G^{-1} G \sim \operatorname{Tr} \mathbf{1}=$ const. To verify this one can check from the stationarity condition (25) that indeed to this order

$$
\frac{\delta \Gamma[\phi, G]}{\delta G} \simeq-\frac{i}{2} G^{-1}+\frac{i}{2} G_{0}^{-1}(\phi)=-\frac{1}{2} R \Rightarrow G^{-1}=G_{0}^{-1}(\phi)-i R .
$$

To go beyond this order it is convenient to write the exact $\Gamma[\phi, G]$ as the one-loop type expression (27) and a 'rest':

$$
\Gamma[\phi, G]=S[\phi]+\frac{i}{2} \operatorname{Tr} \ln G^{-1}+\frac{i}{2} \operatorname{Tr} G_{0}^{-1}(\phi) G+\Gamma_{2}[\phi, G]+\text { const }
$$

Here we have added an irrelevant constant which can be adjusted for normalization. To get an understanding of the 'rest' term $\Gamma_{2}[\phi, G]$ we vary this expression with respect to $G$, which yields

$$
G_{a b}^{-1}(x, y)=G_{0, a b}^{-1}(x, y ; \phi)-i R_{a b}(x, y)-\Sigma_{a b}(x, y ; \phi, G),
$$


where we have written

$$
\Sigma_{a b}(x, y ; \phi, G) \equiv 2 i \frac{\delta \Gamma_{2}[\phi, G]}{\delta G_{a b}(x, y)}
$$

Comparing with the exact expression (19) one observes that

$$
\Sigma_{a b}(x, y ; \phi, G)=\Sigma_{a b}^{R}(x, y ; \phi),
$$

which relates the functional $G$-derivative of $\Gamma_{2}[\phi, G]$ to the proper self-energy. As mentioned above, to the proper self-energy only 1 PI diagrams contribute with propagator lines associated to the effective classical propagator $\left(G_{0}^{-1}-i R\right)^{-1}$. The mapping between $\Sigma_{a b}(x, y ; \phi, G)$ and $\Sigma_{a b}^{R}(x, y ; \phi)$ is provided by (29), which can be used to express the full propagator $G$ as an infinite series in terms of the classical propagator $G_{0}$ and $\Sigma$ :

$$
\begin{aligned}
G= & \left(G_{0}^{-1}-i R\right)^{-1}+\left(G_{0}^{-1}-i R\right)^{-1} \Sigma\left(G_{0}^{-1}-i R\right)^{-1} \\
& +\left(G_{0}^{-1}-i R\right)^{-1} \Sigma\left(G_{0}^{-1}-i R\right)^{-1} \Sigma\left(G_{0}^{-1}-i R\right)^{-1}+\ldots
\end{aligned}
$$

where we employ an obvious matrix notation. As a consequence there is a direct correspondence between a 1PI diagram with propagator lines associated to $G$ and an infinite set of 1PI diagrams with propagator lines associated to the classical propagator $G_{0}$. Below we will consider some explicit examples. Most importantly, from the fact that to $\Sigma(\phi, G)$ only 1PI diagrams contribute one can conclude that

- $\Gamma_{2}[\phi, G]$ only contains contributions from two-particle irreducible (2PI) diagrams.

A diagram is said to be two-particle irreducible if it does not become disconnected by opening two lines. Suppose $\Gamma_{2}[\phi, G]$ had a two-particle reducible contribution. The latter could be written as $\tilde{\Gamma} G G \tilde{\Gamma}^{\prime}$, where $G G$ denotes in a matrix notation two propagator lines connecting two parts $\tilde{\Gamma}$ and $\tilde{\Gamma}^{\prime}$ of a diagram. Then $\Sigma(\phi, G)$ would contain a contribution of the form $\tilde{\Gamma} G \tilde{\Gamma}^{\prime}$ since it is given by a derivative of $\Gamma_{2}$ with respect to $G$. Such a structure is one-particle reducible and cannot occur for the proper self-energy. Therefore two-particle reducible contributions to $\Gamma_{2}[\phi, G]$ are absent.

Diagrammatically, the graphs contributing to $\Sigma(\phi, G)$ are obtained by opening one propagator line in graphs contributing to $\Gamma_{2}[\phi, G]$. This is exemplified for a two- and a three-loop graph for $\Gamma_{2}$ and the corresponding self-energy graphs:

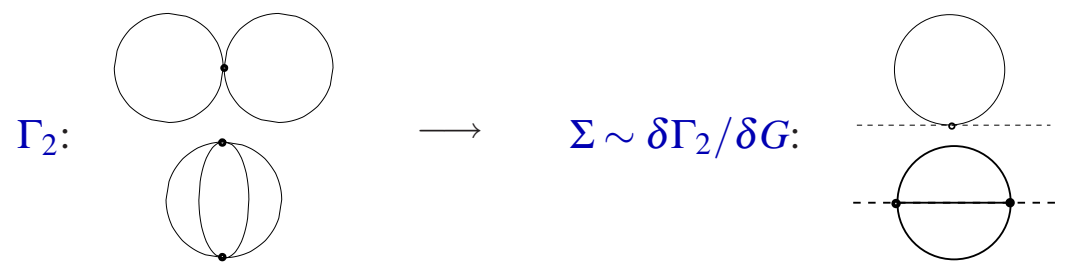

Because of (32) each 2PI diagram corresponds to infinite series of 1PI diagrams, and the above 2PI two- and three-loop diagrams contain e.g. the full series of so-called "daisies" and "ladder" resummations: 


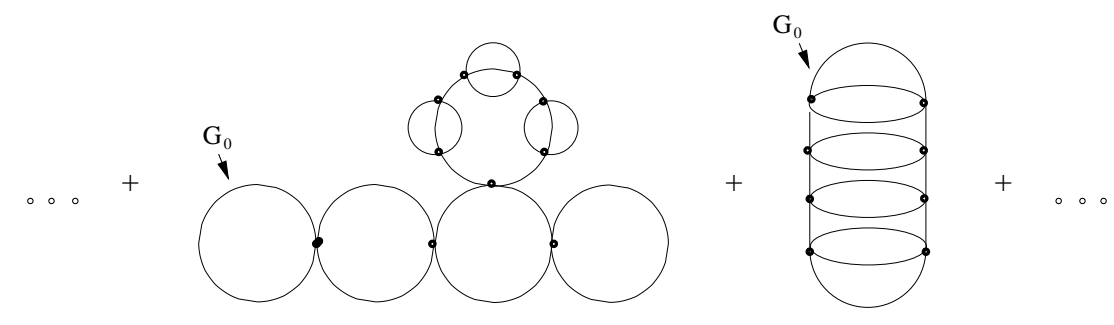

\subsection{Loop or coupling expansion of the 2PI effective action}

Loop or coupling expansions of the 2PI effective action proceed along the same lines as the corresponding expansions for the standard 1PI effective action, with the only difference that

- the full propagator $G$ is associated to propagator lines of a diagram

- and only 2PI graphs are kept.

To be specific, for the considered $N$-component scalar field theory the diagrams are constructed in the standard way from the effective interaction

$$
i S_{\mathrm{int}}[\phi, \varphi]=-\int_{x} i \frac{\lambda}{6 N} \phi_{a}(x) \varphi_{a}(x) \varphi_{b}(x) \varphi_{b}(x)-\int_{x} i \frac{\lambda}{4 ! N}\left(\varphi_{a}(x) \varphi_{a}(x)\right)^{2},
$$

which is obtained from the classical action (13) by shifting $\varphi_{a}(x) \rightarrow \phi_{a}(x)+\varphi_{a}(x)$ and collecting all terms cubic and quartic in the fluctuating field $\varphi_{a}(x)$. As for the 1PI effective action, in addition to the quartic interaction there is an effective cubic interaction for non-vanishing field expectation value, i.e. $\phi_{a}(x) \neq 0$.

Since $\Gamma[\phi, G]$ is a functional, which associates a number to the fields $\phi$ and $G$, only closed loop diagrams can appear. We consider for a moment the real scalar theory for $N=1$. To lowest order one has $\Gamma_{2}[\phi, G]=0$ and we recover the one-loop result given in Eq. (27). At two-loop order there are two contributions

$$
\begin{aligned}
\Gamma_{2}^{(2 \text { loop })}[\phi, G]= & -i 3\left(-i \frac{\lambda}{4 !}\right) \int_{x} G^{2}(x, x) \\
& -i 6 \frac{1}{2} \int_{x y}\left(-i \frac{\lambda}{6} \phi(x)\right)\left(-i \frac{\lambda}{6} \phi(y)\right) G^{3}(x, y),
\end{aligned}
$$

where we have made explicit the different factors coming from the overall $-i$ in the defining functional integral for $\Gamma[\phi, G]$ (cf. Eq. (14)), the combinatorics and the vertices. The 2PI loop expansion exhibits of course much less topologically distinct diagrams than the respective 1PI expansion. For instance, in the symmetric phase $(\phi=0)$ one finds that up to four loops only a single diagram contributes at each order. At fifth order there are two distinct diagrams which are shown along with the lower-loop graphs in Fig. 1. For later discussion, we give here also the results up to five loops for general $N$. For $\phi=0$ by virtue of $O(N)$ rotations the propagator can be taken to be diagonal:

$$
G_{a b}(x, y)=G(x, y) \delta_{a b} .
$$




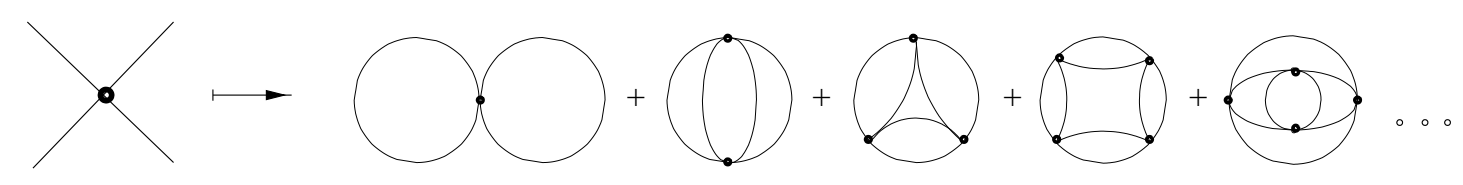

FIGURE 1. Topologically distinct diagrams in the 2PI loop expansion up to five-loop order for $\phi=0$. The suppressed prefactors are given in Eq. (37).

One finds to five-loop order:

$$
\begin{aligned}
& \Gamma_{2}^{(5 \text { loop })}[G]_{\mid G_{a b}=G \delta_{a b}}=\sum_{l=2}^{5} \Gamma_{2}^{(l)} \\
& \Gamma_{2}^{(2)}=-\frac{\lambda}{8} \frac{(N+2)}{3} \int_{x} G^{2}(x, x) \quad, \quad \Gamma_{2}^{(3)}=\frac{i \lambda^{2}}{48} \frac{(N+2)}{3 N} \int_{x y} G^{4}(x, y), \\
& \Gamma_{2}^{(4)}=\frac{\lambda^{3}}{48} \frac{(N+2)(N+8)}{27 N^{2}} \int_{x y z} G^{2}(x, y) G^{2}(x, z) G^{2}(z, y), \\
& \Gamma_{2}^{(5)}=-\frac{i \lambda^{4}}{128} \frac{(N+2)\left(N^{2}+6 N+20\right)}{81 N^{3}} \int_{x y z w} G^{2}(x, y) G^{2}(y, z) G^{2}(z, w) G^{2}(w, x) \\
& -\frac{i \lambda^{4}}{32} \frac{(N+2)(5 N+22)}{81 N^{3}} \int_{x y z w} G^{2}(x, y) G(x, z) G(x, w) G^{2}(z, w) G(y, z) G(y, w) .
\end{aligned}
$$

\subsection{Renormalization}

The 2 PI resummed effective action $\Gamma[\phi, G(\phi)]$ is defined in a standard way by suitable regularization, as e.g. lattice regularization or dimensional regularization, and renormalization conditions which specify the field theory to be considered. We employ renormalization conditions for the two-point function, $\Gamma^{(2)}$, and four-point function, $\Gamma^{(4)}$, given by

$$
\begin{aligned}
\Gamma^{(2)}(x, y) & \left.\equiv \frac{\delta^{2} \Gamma[\phi, G(\phi)]}{\delta \phi(x) \delta \phi(y)}\right|_{\phi=0}, \\
\Gamma^{(4)}(x, y, z, w) & \left.\equiv \frac{\delta^{4} \Gamma[\phi, G(\phi)]}{\delta \phi(x) \delta \phi(y) \delta \phi(z) \delta \phi(w)}\right|_{\phi=0},
\end{aligned}
$$

where we consider a one-component field for notational simplicity. Without loss of generality we use renormalization conditions for $\phi=0$ which in Fourier space read:

$$
\begin{aligned}
\left.Z \Gamma^{(2)}\left(p^{2}\right)\right|_{p=0} & =-m_{R}^{2}, \\
\left.Z \frac{d}{d p^{2}} \Gamma^{(2)}\left(p^{2}\right)\right|_{p=0} & =-1, \\
\left.Z^{2} \Gamma^{(4)}\left(p_{1}, p_{2}, p_{3}\right)\right|_{p_{1}=p_{2}=p_{3}=0} & =-\lambda_{R},
\end{aligned}
$$


with the wave function renormalization $Z{ }^{4}$ Here the renormalized mass parameter $m_{R}$ corresponds to the inverse correlation length. The physical four-vertex at zero momentum is given by $\lambda_{R}$.

\subsubsection{PI renormalization scheme to order $\lambda_{R}^{2}$}

In order to impose the renormalization conditions (39)-(41) one first has to calculate the solution for the two-point field $G(\phi)$ for $\phi=0$, which encodes the resummation and which is obtained from the stationarity condition for the 2PI effective action (25).

The renormalized field is

$$
\phi_{R}=Z^{-1 / 2} \phi .
$$

It is convenient to introduce the counterterms relating the bare and renormalized variables in a standard way with

$$
Z m^{2}=m_{R}^{2}+\delta m^{2}, \quad Z^{2} \lambda=\lambda_{R}+\delta \lambda, \quad \delta Z=Z-1,
$$

and we write

$$
G(\phi)=Z G_{R}\left(\phi_{R}\right)
$$

In terms of the renormalized quantities the classical action (13) reads

$$
S=\int_{x}\left(\frac{1}{2} \partial_{\mu} \phi_{R} \partial^{\mu} \phi_{R}-\frac{1}{2} m_{R}^{2} \phi_{R}^{2}-\frac{\lambda R}{24} \phi_{R}^{4}+\frac{1}{2} \delta Z \partial_{\mu} \phi_{R} \partial^{\mu} \phi_{R}-\frac{1}{2} \delta m^{2} \phi_{R}^{2}-\frac{\delta \lambda}{24} \phi_{R}^{4}\right) .
$$

Similarly, one can write for the one-loop part $\operatorname{Tr} \ln G^{-1}=\operatorname{Tr} \ln G_{R}^{-1}$ up to an irrelevant constant and with $G=G(\phi)$ :

$$
\begin{aligned}
\frac{i}{2} \operatorname{Tr} G_{0}^{-1}(\phi) G(\phi)= & -\left.\frac{1}{2} \int_{x}\left(\square_{x}+m_{R}^{2}+\delta Z_{1} \square_{x}+\delta m_{1}^{2}\right) G_{R}\left(x, y ; \phi_{R}\right)\right|_{x=y} \\
& -\frac{\lambda_{R}+\delta \lambda_{1}}{4} \int_{x} \phi_{R}^{2}(x) G_{R}\left(x, x ; \phi_{R}\right) .
\end{aligned}
$$

Here $\delta Z_{1}, \delta m_{1}^{2}$ and $\delta \lambda_{1}$ denote the same counterterms as introduced in (43), however, approximated to the given order. To express $\Gamma_{2}$ in terms of renormalized quantities it is useful to note the identity

$$
\left.\Gamma_{2}[\phi, G(\phi)]\right|_{\lambda}=\left.\Gamma_{2}\left[\phi_{R}, G_{R}\left(\phi_{R}\right)\right]\right|_{\lambda_{R}+\delta \lambda},
$$

which simply follows from the standard relation between the number of vertices, lines and fields by counting factors of $Z$. Therefore, one can replace in $\Gamma_{2}$ the bare field and

${ }^{4}$ For the Fourier transform of the $n$-th derivative one has

$$
\Gamma^{(n)}\left(x_{1}, \ldots x_{n}\right)=\int \frac{\mathrm{d}^{4} p_{1}}{(2 \pi)^{4}} e^{-i p_{1} x_{1}} \ldots \int \frac{\mathrm{d}^{4} p_{n}}{(2 \pi)^{4}} e^{-i p_{n} x_{n}}(2 \pi)^{4} \delta^{4}\left(p_{1}+\ldots p_{n}\right) \Gamma^{(n)}\left(p_{1}, \ldots p_{n}\right) .
$$


propagator by the renormalized ones if one replaces bare by renormalized vertices as well. We emphasize that mass and wavefunction renormalization counterterms, $\delta Z$ and $\delta m^{2}$, do not appear explicitly in $\Gamma_{2}$. The counterterms in the classical action (45), in the one-loop term (46) and beyond one-loop contained in $\Gamma_{2}$ have to be calculated for a given approximation of $\Gamma_{2}$. For an explicit example, we consider here the 2PI effective action to order $\lambda_{R}^{2}$ with

$$
\begin{aligned}
\Gamma_{2}\left[\phi_{R}, G_{R}\left(\phi_{R}\right)\right]= & -\frac{1}{8} \lambda_{R} \int_{x} G_{R}^{2}\left(x, x ; \phi_{R}\right)+i \frac{1}{12} \lambda_{R}^{2} \int_{x y} \phi_{R}(x) G_{R}^{3}\left(x, y ; \phi_{R}\right) \phi_{R}(y) \\
& +i \frac{1}{48} \lambda_{R}^{2} \int_{x y} G_{R}^{4}\left(x, y ; \phi_{R}\right)-\frac{1}{8} \delta \lambda_{2} \int_{x} G_{R}^{2}\left(x, x ; \phi_{R}\right),
\end{aligned}
$$

where the last term contains the respective coupling counterterm at two-loop. There are no three-loop counterterms since the divergences arising from the three-loop contribution in (48) are taken into account by the lower counterterms.

One first has to calculate the solution $G_{R}\left(\phi_{R}\right)$ obtained from the stationarity condition (25) for the 2PI effective action. For this one has to impose the same renormalization condition as for the propagator (39) in Fourier space:

$$
\left.i G_{R}^{-1}\left(p^{2} ; \phi_{R}\right)\right|_{p=0, \phi_{R}=0}=-m_{R}^{2},
$$

for given finite renormalized "four-point" field

$$
\left.V_{R}(x, y ; z, w) \equiv \frac{\delta^{2} i G_{R}^{-1}\left(x, y ; \phi_{R}\right)}{\delta \phi_{R}(z) \phi_{R}(w)}\right|_{\phi_{R}=0} .
$$

For the above approximation we note the identity

$$
\left.\left.\frac{\delta^{2} \Gamma\left[\phi_{R}, G_{R}\left(\phi_{R}\right)\right]}{\delta \phi_{R}(x) \phi_{R}(y)}\right|_{\phi_{R}=0} \equiv i G_{R}^{-1}\left(x, y ; \phi_{R}\right)\right|_{\phi_{R}=0}
$$

for

$$
\delta Z=\delta Z_{1} \quad, \quad \delta m^{2}=\delta m_{1}^{2} \quad, \quad \delta \lambda_{1}=\delta \lambda_{2},
$$

such that (49) for $G_{R}$ is trivially fulfilled because of (39). In contrast to the exact theory, for the 2PI effective action to order $\lambda_{R}^{2}$ a similar identity does not connect the proper four-vertex with $V_{R}{ }^{5}$ Here the respective condition for the four-point field $V_{R}$ in Fourier space reads

$$
\left.V_{R}\left(p_{1}, p_{2}, p_{3}\right)\right|_{p_{1}=p_{2}=p_{3}=0}=-\lambda_{R} \text {. }
$$

Note that this has to be the same than for the four-vertex (41). For the universality class of the $\phi^{4}$ theory there are only two independent input parameters, which we

\footnotetext{
${ }^{5}$ We emphasize that for more general approximations the equation (51) may only be valid up to higher order corrections as well. This is a typical property of self-consistent resummations, and it does not affect the renormalizability of the theory. In this case the proper renormalization procedure still involves, in particular, the conditions (49) and (53).
} 
take to be $m_{R}$ and $\lambda_{R}$, and for the exact theory $V_{R}$ and the four-vertex agree. The renormalization conditions (39)-(41) for the propagator and four-vertex, together with the scheme (49)-(53) provides an efficient fixing of all the above counter terms. In particular, it can be very conveniently implemented numerically, which turns out to be crucial for calculations beyond order $\lambda_{R}$.

We emphasize that the approximation (48) for the 2PI effective action can only be expected to be valid for sufficiently small $\phi_{R} \ll m_{R} / g_{R}$. If the latter is not fulfilled there are additional $\mathscr{O}\left(\lambda_{R}^{2}\right)$ contributions at three-loop $\sim \lambda_{R}^{3} \phi_{R}^{2}$ and $\sim \lambda_{R}^{4} \phi_{R}^{4}$. This approximation should therefore not be used to study the theory in the spontaneously broken phase or near the critical temperature of the second-order phase transition. Quantitative studies of the latter can be performed in practice using $1 / N$ expansion of the 2 PI effective action to NLO.

\subsubsection{Renormalized equations for the two- and four-point functions}

From the 2PI effective action to order $\lambda_{R}^{2}$ we find with $\delta \lambda_{1}=\delta \lambda_{2}$ from (52) for the two-point function:

$$
\begin{aligned}
i G_{R}^{-1}\left(x, y ; \phi_{R}\right)= & -\left[\left(1+\delta Z_{1}\right) \square_{x}+m_{R}^{2}+\delta m_{1}^{2}\right. \\
& \left.+\frac{1}{2}\left(\lambda_{R}+\delta \lambda_{1}\right)\left(G_{R}\left(x, x ; \phi_{R}\right)+\phi_{R}^{2}(x)\right)\right] \delta(x-y) \\
& +\frac{i}{2} \lambda_{R}^{2} G_{R}^{2}\left(x, y ; \phi_{R}\right) \phi_{R}(x) \phi_{R}(y)+\frac{i}{6} \lambda_{R}^{2} G_{R}^{3}\left(x, y ; \phi_{R}\right) .
\end{aligned}
$$

According to (51) this expression coincides with the one for the propagator $\delta^{2} \Gamma\left[\phi_{R}, G_{R}\left(\phi_{R}\right)\right] / \delta \phi_{R}(x) \delta \phi_{R}(y)$ at $\phi_{R}=0$. It is straightforward to verify this using

$$
\frac{\delta \Gamma\left[\phi_{R}, G_{R}\left(\phi_{R}\right)\right]}{\delta \phi_{R}(x)} \equiv \frac{\delta \Gamma\left[\phi_{R}, G_{R}\right]}{\delta \phi_{R}(x)},
$$

which is valid since the variation of $G_{R}\left(\phi_{R}\right)$ with $\phi_{R}$ does not contribute due to the stationarity condition (25). The four-point field (50) in this approximation is given by

$$
\begin{aligned}
V_{R}(x, y ; z, w)= & -\left(\lambda_{R}+\delta \lambda_{1}\right) \delta(x-y) \delta(x-z) \delta(x-w) \\
& -\left.\frac{1}{2}\left(\lambda_{R}+\delta \lambda_{1}\right) \frac{\delta^{2} G_{R}\left(x, x ; \phi_{R}\right)}{\delta \phi_{R}(z) \delta \phi_{R}(w)}\right|_{\phi_{R}=0} \delta(x-y) \\
& +\frac{i}{2} \lambda_{R}^{2}(\delta(x-w) \delta(y-z)+\delta(y-w) \delta(x-z) \\
& \left.+\left.\frac{\delta^{2} G_{R}\left(x, y ; \phi_{R}\right)}{\delta \phi_{R}(z) \delta \phi_{R}(w)}\right|_{\phi_{R}=0}\right) G_{R}^{2}\left(x, y ; \phi_{R}=0\right) .
\end{aligned}
$$


Inserting the chain rule formula

$$
\left.\frac{\delta^{2} i G_{R}\left(x, y ; \phi_{R}\right)}{\delta \phi_{R}(z) \delta \phi_{R}(w)}\right|_{\phi_{R}=0}=-\left.\int_{u, v} G_{R}\left(x, u ; \phi_{R}\right) V_{R}(u, v ; z, w) G_{R}\left(v, y ; \phi_{R}\right)\right|_{\phi_{R}=0}
$$

one observes that Eqs. (56) and (54) form a closed set of equations for the determination of the counterterms $\delta Z_{1}, \delta m_{1}^{2}$ and $\delta \lambda_{1}$. Together with (52) one notes that $\delta \lambda$ would be undetermined from these equations alone. This counterterm is determined by taking into account the equation for the physical four-vertex, which is obtained from the above $2 \mathrm{PI}$ effective action as

$$
\begin{array}{r}
\left.\frac{\delta^{4} \Gamma\left[\phi_{R}, G_{R}\left(\phi_{R}\right)\right]}{\delta \phi_{R}(x) \delta \phi_{R}(y) \delta \phi_{R}(z) \delta \phi_{R}(w)}\right|_{\phi_{R}=0}=-\left(\lambda_{R}+\delta \lambda\right) \delta(x-y) \delta(x-z) \delta(x-w) \\
+V_{R}(x, y ; z, w)+V_{R}(x, z ; y, w)+V_{R}(x, w ; y, z) \\
-\left.2\left(\frac{\delta i G_{R}^{-1}\left(x, y ; \phi_{R}\right)}{\delta G_{R}(z, w)}+\frac{\delta i G_{R}^{-1}\left(x, z ; \phi_{R}\right)}{\delta G_{R}(y, w)}+\frac{\delta i G_{R}^{-1}\left(x, w ; \phi_{R}\right)}{\delta G_{R}(y, z)}\right)\right|_{\phi_{R}=0}
\end{array}
$$

where from (54) one uses the relation

$$
\begin{aligned}
\left.\frac{\delta i G_{R}^{-1}\left(x, y ; \phi_{R}\right)}{\delta G_{R}(z, w)}\right|_{\phi_{R}=0}= & -\frac{1}{2}\left(\lambda_{R}+\delta \lambda_{1}\right) \delta(x-y) \delta(x-z) \delta(x-w) \\
& +\frac{i}{2} \lambda_{R}^{2} G_{R}^{2}\left(x, y ; \phi_{R}=0\right) \delta(x-z) \delta(y-w) .
\end{aligned}
$$

We emphasize that the counterterm $\delta \lambda$ plays a crucial role in the broken phase, since it is always multiplied by the field $\phi_{0}$ and hence it is essential for the determination of the effective potential. It is also required in the symmetric phase, in particular, when one calculates the momentum-dependent four-vertex using Eq. (58).

It is instructive to consider for a moment the 2PI effective action to two-loop order for which much can be discussed analytically. In this case $Z=1$ and the renormalized vacuum mass $m_{R}$ of Eq. (39) or, equivalently, of Eq. (49) is given to this order by

$$
\begin{aligned}
\frac{m_{R}^{2}}{\lambda} & =\frac{1}{2} \mu^{\varepsilon} \int \frac{\mathrm{d}^{d} k}{(2 \pi)^{d}}\left(k^{2}+m_{R}^{2}\right)^{-1}+\frac{m^{2}}{\lambda} \\
& =-\frac{m_{R}^{2}}{16 \pi^{2}}\left(\frac{1}{\varepsilon}-\ln \frac{m_{R}}{\bar{\mu}}+\frac{1}{2}\right)+\frac{m^{2}}{\lambda}
\end{aligned}
$$

where $m^{2}=m_{R}^{2}+\delta m_{1}^{2}$ and $\lambda=\lambda_{R}+\delta \lambda_{1}$ and we have used (52). Here we have employed dimensional regularization and evaluated the integral in $d=4-\varepsilon$ for Euclidean momenta $k$. The bare coupling in the action (13) has been rescaled accordingly, $\lambda \rightarrow \mu^{\varepsilon} \lambda$, and is dimensionless; $\bar{\mu}^{2} \equiv 4 \pi e^{-\gamma_{E}} \mu^{2}$ and $\gamma_{E}$ denotes Euler's constant. Below we will consider lattice regularizations for comparison and to go beyond two-loop order. 
Similarly, the zero-temperature four-point function resulting from Eq. (53) for the 2PI effective action to order $\lambda_{R}$ is given by

$$
\begin{aligned}
\lambda_{R} & =\lambda-\frac{\lambda \lambda_{R}}{2} \mu^{\varepsilon} \int \frac{\mathrm{d}^{d} k}{(2 \pi)^{d}}\left(k^{2}+m_{R}^{2}\right)^{-2} \\
& =\lambda-\frac{\lambda \lambda_{R}}{16 \pi^{2}}\left(\frac{1}{\varepsilon}-\ln \frac{m_{R}}{\bar{\mu}}\right)
\end{aligned}
$$

with (52). We emphasize that the same zero-temperature equation is obtained starting from the renormalization condition for the proper four-vertex (41) with $\delta \lambda=3 \delta \lambda_{1}$. One observes that all counterterms are uniquely fixed by the renormalization procedure discussed in the previous section.

Though dimensional regularization is elegant for analytical computations, it turns out that high momentum cutoff regularizations are often more efficient for numerical implementations. We will discuss below cutoff regularizations that are obtained by formulating the theory on a discrete space-time lattice. In particular, it is often convenient to carry out the numerical calculations using unrenormalized equations, or equations where only the dominant (quadratically) divergent contributions in the presence of scalars are subtracted. In order to obtain results that are insensitive to variations of the cutoff, it is then typically sufficient to consider momentum cutoffs that are sufficiently large compared to the characteristic energy-momentum scale of the process of interest. It should be also stressed that a number of important renormalized quantities such as renormalized masses or damping rates can be directly inferred from the oscillation frequency or the damping of the time-evolution for the unrenormalized propagator $G$.

\subsection{PI effective action for fermions}

The construction of the 2PI effective action for fermionic fields proceeds along very similar lines than for bosons. However, one has to take into account the anti-commuting (Grassmann) behavior of the fermion fields. The main differences compared to the bosonic case can be already observed from the one-loop part $\left(\Gamma_{2} \equiv 0\right)$ of the 2PI effective action. For vanishing field expectation values it involves the integrals:

$$
\begin{aligned}
& \text { Fermions: }-i \ln \int \mathscr{D} \bar{\psi} \mathscr{D} \psi e^{i S_{0}^{(f)}}=-i \ln \left(\operatorname{det} \Delta_{0}^{-1}\right)=-i \operatorname{Tr} \ln \Delta_{0}^{-1}, \\
& \text { Bosons: } \quad-i \ln \int \mathscr{D} \varphi e^{i S_{0}}=-i \ln \left(\operatorname{det} G_{0}^{-1}\right)^{-\frac{1}{2}}=\frac{i}{2} \operatorname{Tr} \ln G_{0}^{-1}
\end{aligned}
$$

where $S_{0}^{(f)}=\int \mathrm{d}^{4} x \mathrm{~d}^{4} y \bar{\psi}(x) i \Delta_{0}^{-1}(x, y) \psi(y)$ denotes a fermion action that is bilinear in the Grassmann fields. For Dirac fermions with mass $m^{(f)}$ the free inverse propagator reads

$$
i \Delta_{0}^{-1}(x, y)=\left[i \partial_{x}-m^{(f)}\right] \delta(x-y),
$$

where $\not \partial \equiv \gamma^{\mu} \partial_{\mu}$ with Dirac matrices $\gamma_{\mu}(\mu=0, \ldots, 3)$, and $\bar{\psi}=\psi^{\dagger} \gamma^{0}$. For the bosons $S_{0}$ is given by the quadratic part of (13). Comparing the two integrals one observes that the 
factor $1 / 2$ for the bosonic case is replaced by -1 for the fermion fields because of their anti-commuting property. With this difference, following along the lines of Sec. 2 one finds that the 2PI effective action for fermions can be written in complete analogy to (28). Accordingly, for the case of vanishing fermion field expectation values, $\langle\Psi\rangle=\langle\bar{\Psi}\rangle=0$, one has:

$$
\Gamma[\Delta]=-i \operatorname{Tr} \ln \Delta^{-1}-i \operatorname{Tr} \Delta_{0}^{-1} \Delta+\Gamma_{2}[\Delta]+\mathrm{const}
$$

Here $\Gamma_{2}[\Delta]$ contains all 2PI diagrams with lines associated to the time ordered propagator $\Delta(x, y)=\langle T \Psi(x) \bar{\Psi}(y)\rangle$. As for the 1PI effective action diagrams get an additional minus sign from each closed fermion loop. The trace "Tr" includes integration over time and spatial coordinates, as well as summation over field indices.

As for the bosonic case of Eq. (25), the equation of motion for $\Delta$ in absence of external sources is obtained by extremizing the effective action:

$$
\frac{\delta \Gamma[\Delta]}{\delta \Delta(x, y)}=0
$$

Using (64) this stationarity condition can be written as

$$
\Delta^{-1}(x, y)=\Delta_{0}^{-1}(x, y)-\Sigma^{(f)}(x, y ; \Delta),
$$

with the proper fermion self-energy:

$$
\Sigma^{(f)}(x, y ; \Delta) \equiv-i \frac{\delta \Gamma_{2}[\Delta]}{\delta \Delta(y, x)}
$$

\subsubsection{Chiral quark-meson model}

As an example we consider a quantum field theory involving two fermion flavors ("quarks") coupled in a chirally invariant way to a scalar $\sigma$-field and a triplet of pseudoscalar "pions" $\pi^{a}(a=1,2,3)$. The classical action reads

$$
\begin{aligned}
S=\int \mathrm{d}^{4} x & \left\{\bar{\psi} i \not \partial \psi+\frac{1}{2}\left[\partial_{\mu} \sigma \partial^{\mu} \sigma+\partial_{\mu} \pi^{a} \partial^{\mu} \pi^{a}\right]\right. \\
+ & \left.\frac{h}{N_{f}} \bar{\psi}\left[\sigma+i \gamma_{5} \tau^{a} \pi^{a}\right] \psi-V\left(\sigma^{2}+\pi^{2}\right)\right\},
\end{aligned}
$$

where $\pi^{2} \equiv \pi^{a} \pi^{a}$. Here $\tau^{a}$ denote the standard Pauli matrices and $h / N_{f}$ is the Yukawa coupling. The number of fermion flavors is $N_{f} \equiv 2$ and $N_{f}^{2}$ is the number of scalar components. The above action is invariant under chiral $S U_{L}(2) \times S U_{R}(2) \sim O(4)$ transformations. For a quartic scalar self-interaction,

$$
V\left(\sigma^{2}+\pi^{2}\right)=\frac{1}{2} m^{2}\left(\sigma^{2}+\pi^{2}\right)+\frac{\lambda}{4 ! N_{f}^{2}}\left(\sigma^{2}+\pi^{2}\right)^{2},
$$


this model corresponds to the well known linear $\sigma$-model, incorporating the chiral symmetry of massless two-flavor QCD.

Here we do not consider the possibility of a spontaneously broken symmetry, thus field expectation values vanish. For this model the 2PI effective action is then a functional of fermion as well as scalar propagators. The scalar fields form an $O(4)$ vector $\phi_{a}(x) \equiv(\sigma(x), \vec{\pi}(x))$ and we denote the full scalar propagator by $G_{a b}(x, y)$ with $a, b=0, \ldots, 3$. In addition to its Dirac structure the fermion propagator carries flavor indices $i, j$ for $N_{f}=2$ flavors. The 2PI effective action for this Yukawa model is given by

$$
\Gamma[G, \Delta]=\frac{i}{2} \operatorname{Tr} \ln G^{-1}+\frac{i}{2} \operatorname{Tr} G_{0}^{-1} G-i \operatorname{Tr} \ln \Delta^{-1}-i \operatorname{Tr} \Delta_{0}^{-1} \Delta+\Gamma_{2}[G, \Delta]+\text { const },
$$

with the free scalar and fermion inverse propagators

$$
i G_{0, a b}^{-1}(x, y)=-\left(\square_{x}+m^{2}\right) \boldsymbol{\delta}(x-y) \boldsymbol{\delta}_{a b}, i \Delta_{0, i j}^{-1}(x, y)=\left(i \partial_{x}-m^{(f)}\right) \boldsymbol{\delta}(x-y) \boldsymbol{\delta}_{i j}
$$

The equation of motions for the scalar and fermion propagators are obtained from the stationary conditions

$$
\frac{\delta \Gamma[G, \Delta]}{\delta G_{a b}(x, y)}=0 \quad, \quad \frac{\delta \Gamma[G, \Delta]}{\delta \Delta_{i j}(x, y)}=0 .
$$

The first non-zero order in a loop expansion of $\Gamma_{2}[G, \Delta]$ consists of a purely scalar contribution corresponding to the two-loop diagram of Fig. (1), as well as a fermionscalar contribution depicted here graphically:

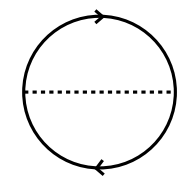

solid line: fermion propagator $\Delta$ dashed line: scalar propagator $G$

Without loss of generality in the absence of spontaneous chiral symmetry breaking, the effective action and its functional derivatives can be evaluated for $G_{a b}$ taken to be the unit matrix in $O(4)$-space. Similarly, the most general fermion two-point function can be taken to be proportional to unity in flavor space and we can write:

$$
G_{a b}(x, y)=G(x, y) \delta_{a b} \quad, \quad \Delta_{i j}(x, y)=\Delta(x, y) \delta_{i j} .
$$

The two-loop approximation then reads

$$
\begin{aligned}
\left.\Gamma_{2}^{(2 \text { loop })}[G, \Delta]\right|_{G_{a b}=G \delta_{a b}, \Delta_{i j}=\Delta \delta_{i j}=} & -\frac{\lambda}{8} \frac{\left(N_{f}^{2}+2\right)}{3} \int_{x} G^{2}(x, x) \\
& -i h^{2} \frac{N_{f}}{2} \int_{x y} \operatorname{tr}[\Delta(x, y) \Delta(y, x)] G(x, y),
\end{aligned}
$$

where the trace "tr" acts only in Dirac space. According to (30) and (67) the self-energies can then be obtained by

$$
\Sigma_{a b}(x, y)=2 i \int_{x^{\prime} y^{\prime}} \frac{\left.\delta \Gamma_{2}\right|_{G \delta_{a b}, \Delta \delta_{i j}}}{\delta G\left(x^{\prime}, y^{\prime}\right)} \frac{\delta G\left(x^{\prime}, y^{\prime}\right)}{\delta G^{a b}(x, y)}=2 i \frac{\delta_{a b}}{N_{f}^{2}} \frac{\left.\delta \Gamma_{2}\right|_{G \delta_{a b}, \Delta \delta_{i j}}}{\delta G(x, y)}
$$




$$
\Sigma_{i j}^{(f)}(x, y)=-i \frac{\delta_{a b}}{N_{f}} \frac{\left.\delta \Gamma_{2}\right|_{G \delta_{a b}, \Delta \delta_{i j}}}{\delta \Delta(y, x)},
$$

where we have used $G=G_{a b} \delta_{a b} / N_{f}^{2}$ with $\delta_{a a}=N_{f}^{2}$, and equivalently for the fermion propagator with $\delta_{i i}=N_{f}$.

\subsection{Two-particle irreducible $1 / N$ expansion}

In this section we discuss a systematic nonperturbative approximation scheme for the 2PI effective action. It classifies the contributions to the 2PI effective action according to their scaling with powers of $1 / N$, where $N$ denotes the number of field components:

$$
\begin{aligned}
\Gamma_{2}[\phi, G]=\Gamma_{2}^{\mathrm{LO}}[\phi, G]+\Gamma_{2}^{\mathrm{NLO}}[\phi, G]+\Gamma_{2}^{\mathrm{NNLO}}[\phi, G]+\ldots \\
\sim N^{1} \sim N^{0} \sim N^{-1}
\end{aligned}
$$

Each subsequent contribution $\Gamma_{2}^{\mathrm{LO}}, \Gamma_{2}^{\mathrm{NLO}}, \Gamma_{2}^{\mathrm{NNLO}}$ etc. is down by an additional factor of $1 / N$. The importance of an expansion in powers of $1 / N$ stems from the fact that it provides a controlled expansion parameter that is not based on weak couplings. It can be applied to describe physics characterized by nonperturbatively large fluctuations, such as encountered near second-order phase transitions in thermal equilibrium, or for extreme nonequilibrium phenomena such as parametric resonance. For the latter cases a 2PI coupling or loop expansion is not applicable. The method can be applied to bosonic or fermionic theories alike if a suitable field number parameter is available, and we exemplify it here for the case of the scalar $O(N)$-symmetric theory with classical action (13). We comment on its application to the chiral quark-meson model below.

\subsubsection{Classification of diagrams}

We present a simple classification scheme based on $O(N)$-invariants which parametrize the 2PI diagrams contributing to $\Gamma[\phi, G]$. The interaction term of the classical action in Eq. (13) is written such that $S[\phi]$ scales proportional to $N$. From the fields $\phi_{a}$ alone one can construct only one independent invariant under $O(N)$ rotations, which can be taken as tr $\phi \phi \equiv \phi^{2}=\phi_{a} \phi_{a} \sim N$. The minimum $\phi_{0}$ of the classical effective potential for this theory is given by $\phi_{0}^{2}=N\left(-6 m^{2} / \lambda\right)$ for negative mass-squared $m^{2}$ and scales proportional to $N$. Similarly, the trace with respect to the field indices of the classical propagator $G_{0}$ is of order $N$.

The 2PI effective action is a singlet under $O(N)$ rotations and parametrized by the two fields $\phi_{a}$ and $G_{a b}$. To write down the possible $O(N)$ invariants, which can be constructed from these fields, we note that the number of $\phi$-fields has to be even in order to construct an $O(N)$-singlet. For a compact notation we use $(\phi \phi)_{a b}=\phi_{a} \phi_{b}$. All functions of $\phi$ and $G$, which are singlets under $O(N)$, can be built from the irreducible (i.e. nonfactorizable in field-index space) invariants

$$
\phi^{2}, \quad \operatorname{tr}\left(G^{n}\right) \quad \text { and } \quad \operatorname{tr}\left(\phi \phi G^{n}\right) .
$$



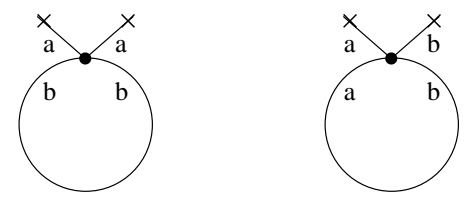

FIGURE 2. Graphical representation of the $\phi$-dependent contributions for $\Gamma_{2} \equiv 0$. The crosses denote field insertions $\sim \phi_{a} \phi_{a}$ for the left figure, which contributes at leading order, and $\sim \phi_{a} \phi_{b}$ for the right figure contributing at next-to-leading order.

We note that for given $N$ only the invariants with $n \leq N$ are irreducible - there cannot be more independent invariants than fields. We will see below that for lower orders in the $1 / N$ expansion and for sufficiently large $N$ one has $n<N$. In particular, for the nextto-leading order approximation one finds that only invariants with $n \leq 2$ appear, which makes the expansion scheme appealing from a practical point of view.

Since each single graph contributing to $\Gamma[\phi, G]$ is an $O(N)$-singlet, we can express them with the help of the set of invariants in Eq. (77). The factors of $N$ in a given graph have two origins:

- each irreducible invariant is taken to scale proportional to $N$ since it contains exactly one trace over the field indices,

- while each vertex provides a factor of $1 / N$.

The expression (28) for the 2PI effective action contains, besides the classical action, the one-loop contribution proportional to $\operatorname{Tr} \ln G^{-1}+\operatorname{Tr} G_{0}^{-1}(\phi) G$ and a nonvanishing $\Gamma_{2}[\phi, G]$ if higher loops are taken into account. The one-loop term contains both leading order (LO) and next-to-leading order (NLO) contributions. The logarithmic term corresponds, in absence of other terms, simply to the free field effective action and scales proportional to the number of field components $N$. To separate the LO and NLO contributions at the one-loop level consider the second term $\operatorname{Tr} G_{0}^{-1}(\phi) G$. From the form of the classical propagator (20) one observes that it can be decomposed into a term proportional to $\operatorname{tr}(G) \sim N$ and terms $\sim(\lambda / 6 N)[\operatorname{tr}(\phi \phi) \operatorname{tr}(G)+2 \operatorname{tr}(\phi \phi G)]$. This can be seen as the sum of two "2PI one-loop graphs" with field insertion $\sim \phi_{a} \phi_{a}$ and $\sim \phi_{a} \phi_{b}$, respectively, as shown in Fig. 2. Counting the factors of $N$ coming from the traces and the prefactor, one sees that only the first contributes at LO, while the second one is NLO.

According to the above rules one draws all topologically distinct 2PI diagrams and counts the number of closed lines as well as the number of lines connecting two field insertions in a diagram following the indices. For instance, the left diagram of Fig. 2 admits one line connecting two field insertions and one closed line. In contrast, the right figure admits only one line connecting two field insertions by following the indices. Therefore, the right graph exhibits one factor of $N$ less and becomes subleading. Similarly, for the two-loop graph below one finds:
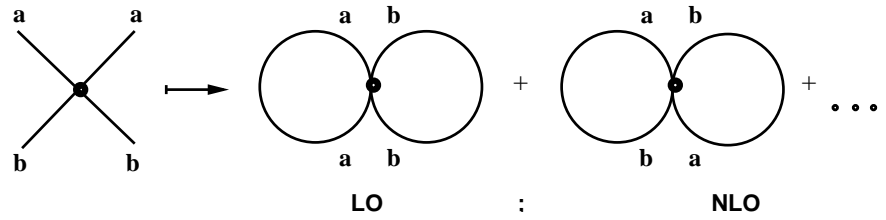


$$
(\operatorname{tr} G)^{2} / N \sim N \quad \operatorname{tr} G^{2} / N \sim N^{0}
$$

The same can be applied to higher orders. We consider first the contributions to $\Gamma_{2}[\phi=0, G] \equiv \Gamma_{2}[G]$, i.e. for a vanishing field expectation value and discuss $\phi \neq 0$ below. The LO contribution to $\Gamma_{2}[G]$ consists of only one two-loop graph, whereas to NLO there is an infinite series of contributions which can be analytically summed:

$$
\begin{aligned}
\Gamma_{2}^{\mathrm{LO}}[G] & =-\frac{\lambda}{4 ! N} \int_{x} G_{a a}(x, x) G_{b b}(x, x), \\
\Gamma_{2}^{\mathrm{NLO}}[G] & =\frac{i}{2} \operatorname{Tr} \ln [B(G)], \\
B(x, y ; G) & =\delta(x-y)+i \frac{\lambda}{6 N} G_{a b}(x, y) G_{a b}(x, y) .
\end{aligned}
$$

In order to see that (79) with (80) sums the following infinite series of diagrams

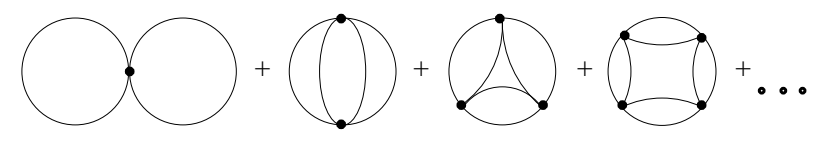

one can expand:

$$
\begin{aligned}
\operatorname{Tr} \ln [B(G)] & =\int_{x}\left(i \frac{\lambda}{6 N} G_{a b}(x, x) G_{a b}(x, x)\right) \\
& -\frac{1}{2} \int_{x y}\left(i \frac{\lambda}{6 N} G_{a b}(x, y) G_{a b}(x, y)\right)\left(i \frac{\lambda}{6 N} G_{a^{\prime} b^{\prime}}(y, x) G_{a^{\prime} b^{\prime}}(y, x)\right) \\
& +\ldots
\end{aligned}
$$

The first term on the r.h.s. of (81) corresponds to the two-loop graph with the index structure exhibiting one trace such that the contribution scales as $\operatorname{tr} G^{2} / N \sim N^{0}$. One observes that each additional contribution scales as well proportional to $\left(\operatorname{tr} G^{2} / N\right)^{n} \sim N^{0}$ for all $n \geq 2$. Thus all terms contribute at the same order.

The terms appearing in the presence of a nonvanishing field expectation value are obtained from the effectively cubic interaction term in (33) for $\phi \neq 0$. One first notes that there is no $\phi$-dependent graph contributing at LO. To NLO there is again an infinite series of diagrams $\sim N^{0}$ which can be summed:

$$
\begin{aligned}
\Gamma_{2}^{\mathrm{LO}}[\phi, G] & \equiv \Gamma_{2}^{\mathrm{LO}}[G] \\
\Gamma_{2}^{\mathrm{NLO}}[\phi, G] & =\Gamma_{2}^{\mathrm{NLO}}[\phi \equiv 0, G]+\frac{i \lambda}{6 N} \int_{x y} I(x, y ; G) \phi_{a}(x) G_{a b}(x, y) \phi_{b}(y), \\
I(x, y ; G) & =\frac{\lambda}{6 N} G_{a b}(x, y) G_{a b}(x, y)-i \frac{\lambda}{6 N} \int_{z} I(x, z ; G) G_{a b}(z, y) G_{a b}(z, y) .
\end{aligned}
$$


The series of terms contained in (83) with (84) corresponds to the diagrams:

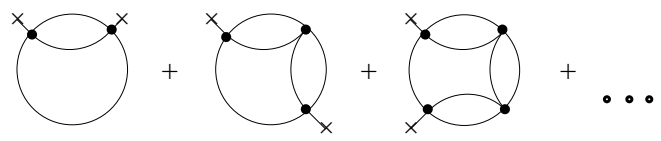

The functions $I(x, y ; G)$ and the inverse of $B(x, y ; G)$ are closely related by

$$
B^{-1}(x, y ; G)=\delta(x-y)-i I(x, y ; G),
$$

which follows from convoluting (80) with $B^{-1}$ and using (84). We note that $B$ and $I$ do not depend on $\phi$, and $\Gamma_{2}[\phi, G]$ is only quadratic in $\phi$ at NLO.

The 2PI can be straightforwardly applied to other theories as well. For instance, the chiral quark-meson model of Sec. 2.3.1 admits a $1 / N_{f}$ expansion. For the latter the LO contribution scales $\sim N_{f}^{2}$ and is given by a purely scalar two-loop term as in Eq. (74). Note that this two-loop contribution contains a LO part $\sim N_{f}^{2}$ as well as a NNLO part $\sim N_{f}^{0}$. At NLO the first fermion contributions to $\Gamma_{2}$ appear, which scale $\sim N_{f}$ (cf. Eq. (74)). If no small coupling is available the expansion parameter $N_{f} \equiv 2$ in this case might not be suitable for a quantitative estimate at low orders. See however the precision tests of Sec. 5 for the scalar $O(N)$ model, which exhibit a good convergence already for moderate values of $N \gtrsim 2$.

\subsubsection{Symmetries and validity of Goldstone's theorem}

We emphasize that by construction each order in the 2PI $1 / N$-expansion respects $O(N)$ symmetry. In particular, this is crucial for the validity of Ward identities. For the case of spontaneous symmetry breaking note that Goldstone's theorem is fulfilled at any order in the 2 PI $1 / N$ expansion. According to (77) the 2 PI effective action can be written as a function of the $O(N)$ invariants:

$$
\Gamma[\phi, G] \equiv \Gamma\left[\phi^{2}, \operatorname{tr}\left(G^{n}\right), \operatorname{tr}\left(\phi \phi G^{p}\right)\right] .
$$

In the case of spontaneous symmetry breaking one has a constant $\phi \neq 0$ and the propagator can be parametrized as

$$
G_{a b}(\phi)=G_{L}\left(\phi^{2}\right) P_{a b}^{L}+G_{T}\left(\phi^{2}\right) P_{a b}^{T}
$$

where $P_{a b}^{L}=\phi_{a} \phi_{b} / \phi^{2}$ and $P_{a b}^{T}=\delta_{a b}-P_{a b}^{L}$ are the longitudinal and transverse projectors with respect to the field direction. The 2 PI resummed effective action $\Gamma[\phi, G(\phi)]$ is obtained by evaluation at the stationary value (25) for $G$. The mass matrix $\mathscr{M}_{a b}$ can then be obtained from

$$
\left.\mathscr{M}_{a b} \sim \frac{\delta^{2} \Gamma[\phi, G(\phi)]}{\delta \phi_{a} \delta \phi_{b}}\right|_{\phi=\mathrm{const}} .
$$

If $\Gamma[\phi, G(\phi)]$ is calculated from (86) and (87) one observes that indeed it depends only on one invariant, $\phi^{2}: \Gamma[\phi, G(\phi)]=\Gamma\left[\phi^{2}\right]$. The form of the mass matrix $\mathscr{M}_{a b}$ can now be 
inferred straightforwardly. To obtain the effective potential $U\left(\phi^{2} / 2\right)$, we write

$$
\left.\Gamma\left[\phi^{2}\right]\right|_{\phi=\text { const }}=\Omega_{d+1} U\left(\phi^{2} / 2\right),
$$

where $\Omega_{d+1}$ is the $d+1$ dimensional Euclidean volume. The expectation value of the field is given by the solution of the stationarity equation (24) which becomes

$$
\frac{\partial U\left(\phi^{2} / 2\right)}{\partial \phi_{a}}=\phi_{a} U^{\prime}=0
$$

where $U^{\prime} \equiv \partial U / \partial\left(\phi^{2} / 2\right)$ and similarly for higher derivatives. The mass matrix reads

$$
\mathscr{M}_{a b}^{2}=\frac{\partial^{2} U\left(\phi^{2} / 2\right)}{\partial \phi_{a} \partial \phi_{b}}=\delta_{a b} U^{\prime}+\phi_{a} \phi_{b} U^{\prime \prime}=\left(U^{\prime}+\phi^{2} U^{\prime \prime}\right) P_{a b}^{L}+U^{\prime} P_{a b}^{T}
$$

In the symmetric phase $\left(\phi_{a}=0\right)$ one finds that all modes have equal mass squared $\mathscr{M}_{a b}^{2}=U^{\prime} \delta_{a b}$. In the broken phase, with $\phi_{a} \neq 0$, Eq. (90) implies that the mass of the transverse modes $\sim U^{\prime}$ vanishes identically in agreement with Goldstone's theorem. Truncations of the 2PI effective action may not show manifestly the presence of massless transverse modes if one considers the solution of the stationarity equation (24) for the two-point field $G$ encoding the resummation. It is important to realize that the $2 \mathrm{PI}$ resummed effective action $\Gamma[\phi, G(\phi)]$ complies fully with the symmetries.

\section{NONEQUILIBRIUM QUANTUM FIELD THEORY}

Out of equilibrium dynamics requires the specification of an initial state. This may include a density matrix at a given time $\rho_{D}\left(t_{0}=0\right)$ in a mixed $\left(\operatorname{Tr} \rho_{D}^{2}(0)<1\right)$ or pure state $\left(\operatorname{Tr} \rho_{D}^{2}(0)=1\right)$. Nonequilibrium means that the initial density matrix does not correspond to a thermal equilibrium density matrix: $\rho_{D}(0) \neq \rho_{D}^{(\mathrm{eq})}$ with for instance $\rho_{D}^{(\mathrm{eq})} \sim e^{-\beta H}$ for the case of a canonical thermal ensemble. Once the initial state is specified, the dynamics can be described in terms of a functional path integral with the classical action $S$ as employed in the previous sections above. The corresponding nonequilibrium effective action is the generating functional for all correlation functions with the initial correlations determined by $\rho_{D}(0)$.

\subsection{Nonequilibrium generating functional}

All information about nonequilibrium quantum field theory is contained in the nonequilibrium generating functional for correlation functions:

$$
Z\left[J, R ; \rho_{D}\right]=\operatorname{Tr}\left\{\rho_{D}(0) T_{\mathscr{C}} e^{i\left(\int_{x} J(x) \Phi(x)+\frac{1}{2} \int_{x y} R(x, y) \Phi(x) \Phi(y)\right)}\right\} .
$$

Here $T_{\mathscr{C}}$ denotes time-ordering along the time path $\mathscr{C}$ appearing in the source term integrals with $\int_{x} \equiv \int_{\mathscr{C}} \mathrm{d} x^{0} \int \mathrm{d}^{d} x$ as specified below. In complete analogy to Eq. (14) we have 
introduced the generating functional with two source terms, $J$ and $R$, in order to construct the corresponding nonequilibrium 2PI effective action below. We suppress field indices in the notation, which can be directly recovered from (14). The nonequilibrium correlation functions, i.e. expectation values of time-ordered products of Heisenberg field operators $\Phi(x)$, are obtained by functional differentiation. For instance the two-point function reads $\left(\operatorname{Tr} \rho_{D}=1\right)$ :

$$
\operatorname{Tr}\left\{\rho_{D}(0) T_{\mathscr{C}} \Phi(x) \Phi(y)\right\} \equiv\left\langle T_{\mathscr{C}} \Phi(x) \Phi(y)\right\rangle=\left.\frac{\delta^{2} Z\left[J, R ; \rho_{D}\right]}{i \delta J(x) i \delta J(y)}\right|_{J=R=0} .
$$

There is a simple functional integral representation of $Z\left[J, R ; \rho_{D}\right]$ similar to (14). One writes the trace as

$$
\begin{aligned}
Z\left[J, R ; \rho_{D}\right]= & \int \mathrm{d} \varphi^{(1)}(\mathbf{x}) \mathrm{d} \varphi^{(2)}(\mathbf{x})\left\langle\varphi^{(1)}\left|\rho_{D}(0)\right| \varphi^{(2)}\right\rangle \\
& \left\langle\varphi^{(2)}\left|T e^{i\left(\int_{x} J(x) \Phi(x)+\frac{1}{2} \int_{x y} R(x, y) \Phi(x) \Phi(y)\right)}\right| \varphi^{(1)}\right\rangle,
\end{aligned}
$$

where the matrix elements are taken with respect to eigenstates of the Heisenberg field operators at intial time, $\Phi(t=0, \mathbf{x})\left|\varphi^{(i)}\right\rangle=\varphi^{(i)}(\mathbf{x})\left|\varphi^{(i)}\right\rangle, i=1,2$. The source-dependent matrix element can be expressed in terms of a path integral using standard techniques if one considers a finite, closed real-time contour $\mathscr{C}$ :

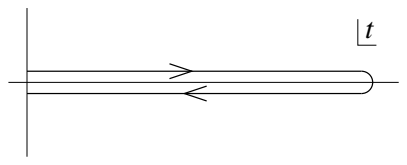

Contour time ordering along this path corresponds to usual time ordering along the forward piece $\mathscr{C}^{+}$and antitemporal ordering on the backward piece $\mathscr{C}^{-}$. Note that any time on $\mathscr{C}^{-}$is considered later than any time on $\mathscr{C}^{+}$. One can then use

$$
\begin{aligned}
& \left\langle\varphi^{(2)}\left|T e^{i\left(\int_{x} J(x) \Phi(x)+\frac{1}{2} \int_{x y} R(x, y) \Phi(x) \Phi(y)\right)}\right| \varphi^{(1)}\right\rangle \\
& =\int_{\varphi^{(2)}(\mathbf{x})=\varphi\left(0^{-}, \mathbf{x}\right)} \mathscr{D}^{\prime} \varphi e^{i\left(S[\varphi]+\int_{x} J(x) \varphi(x)+\frac{1}{2} \int_{x y} R(x, y) \varphi(x) \varphi(y)\right)}, \\
& \varphi^{(1)(\mathbf{x})=\varphi\left(0^{+}, \mathbf{x}\right)}
\end{aligned}
$$

which is the same relation as employed to obtain standard path integral expressions for vacuum or equilibrium matrix elements. Note that the closed time contour starting from the initial time $t_{0}=0$ and ending at $t_{0}$ is required because the density matrix element $\left\langle\varphi^{(1)}\left|\rho_{D}(0)\right| \varphi^{(2)}\right\rangle$ is taken on both sides with respect to states at time $t_{0}$. Using (95) in (94) one finds that the expression for $Z\left[J, R ; \rho_{D}\right]$ directly displays the ingredients entering nonequilibrium quantum field theory - the quantum fluctuations described by the functional integral with action $S$, and the statistical fluctuations encoded in the 
weighted average with the initial-time elements:

$$
Z\left[J, R ; \rho_{D}\right]=\underbrace{\int \mathrm{d} \varphi^{(1)} \mathrm{d} \varphi^{(2)}\left\langle\varphi^{(1)}\left|\rho_{D}(0)\right| \varphi^{(2)}\right\rangle}_{\text {initial conditions }} \underbrace{\int_{\varphi^{(2)}}^{\varphi^{(1)}} \mathscr{D}^{\prime} \varphi e^{i\left(S[\varphi]+\int_{x} J(x) \varphi(x)+\frac{1}{2} \int_{x y} R(x, y) \varphi(x) \varphi(y)\right)}}_{\text {quantum dynamics }}
$$

Of course, this generating functional can be equally applied to standard vacuum physics. In this case the closed time contour just ensures the normalization $Z_{\mid J=R=0}=1$, which can simplify calculations. In particular, the density matrix is time independent for the vacuum as well as for the thermal equilibrium case. In contrast to thermal equilibrium, the nonequilibrium density matrix cannot be interpreted as an evolution operator in imaginary time, as is possible for instance for the canonical $\rho^{(\mathrm{eq})} \sim e^{-\beta H}$.

In the literature formulations of closed time path generating functionals often exhibit an infinite time interval ] $-\infty, \infty$ [. In this case the number of field labels has to be doubled to distinguish the fields on the underlying closed contour. Causality implies that for any $n$-point function with finite time arguments contributions of an infinite time path cancel for times exceeding the largest time argument of the $n$-point function. To avoid unnecessary cancellations of infinite time path contributions we always consider finite time paths. The largest time of the path is kept as a parameter and is evolved in the time evolution equations as described below. We stress that the initial time of the path has to be finite - a system that can thermalize will be already in equilibrium at any finite time if the initial time is sent to the infinite past.

\subsection{Initial conditions}

To understand in more detail how the initial density matrix enters calculations, we consider first the example of a Gaussian density matrix whose most general form can be written as

$$
\begin{aligned}
& \left\langle\varphi^{(1)}\left|\rho_{D}(0)\right| \varphi^{(2)}\right\rangle= \\
& \frac{1}{\sqrt{2 \pi \xi^{2}}} \exp \left\{i \dot{\phi}\left(\varphi^{(1)}-\varphi^{(2)}\right)-\frac{\sigma^{2}+1}{8 \xi^{2}}\left[\left(\varphi^{(1)}-\phi\right)^{2}+\left(\varphi^{(2)}-\phi\right)^{2}\right]\right. \\
& \left.+i \frac{\eta}{2 \xi}\left[\left(\varphi^{(1)}-\phi\right)^{2}-\left(\varphi^{(2)}-\phi\right)^{2}\right]+\frac{\sigma^{2}-1}{4 \xi^{2}}\left(\varphi^{(1)}-\phi\right)\left(\varphi^{(2)}-\phi\right)\right\},
\end{aligned}
$$

where we neglect the spatial dependencies for a moment. Note that for homogeneous field expectation values taking into account spatial dependencies simply amounts to adding a momentum label in Fourier space (cf. also Sec. 3.4.3). In order to see that this is the most general Gaussian density matrix, we first note that (97) is equivalent to the following set of initial conditions for one- and two-point functions:

$$
\phi=\operatorname{Tr}\left\{\rho_{D}(0) \Phi(t)\right\}_{\mid t=0} \quad, \quad \dot{\phi}=\operatorname{Tr}\left\{\rho_{D}(0) \partial_{t} \Phi(t)\right\}_{\mid t=0},
$$




$$
\begin{aligned}
\xi^{2} & =\operatorname{Tr}\left\{\rho_{D}(0) \Phi(t) \Phi\left(t^{\prime}\right)\right\}_{\mid t=t^{\prime}=0}-\phi \phi \\
\xi \eta & =\frac{1}{2} \operatorname{Tr}\left\{\rho_{D}(0)\left(\partial_{t} \Phi(t) \Phi\left(t^{\prime}\right)+\Phi(t) \partial_{t^{\prime}} \Phi\left(t^{\prime}\right)\right)\right\}_{\mid t=t^{\prime}=0}-\dot{\phi} \phi \\
\eta^{2}+\frac{\sigma^{2}}{4 \xi^{2}} & =\operatorname{Tr}\left\{\rho_{D}(0) \partial_{t} \Phi(t) \partial_{t^{\prime}} \Phi\left(t^{\prime}\right)\right\}_{\mid t=t^{\prime}=0}-\dot{\phi} \dot{\phi} .
\end{aligned}
$$

It is straightforward to check explicitly the equivalence between the initial density matrix and the initial conditions for the correlators:

$$
\begin{aligned}
\operatorname{Tr} \rho_{D}(0) & =\int_{-\infty}^{\infty} \mathrm{d} \varphi\left\langle\varphi\left|\rho_{D}(0)\right| \varphi\right\rangle \\
& =\frac{1}{\sqrt{2 \pi \xi^{2}}} \int_{-\infty}^{\infty} \mathrm{d} \varphi \exp \left\{-\frac{1}{2 \xi^{2}}(\varphi-\phi)^{2}\right\}=1, \\
\operatorname{Tr}\left\{\rho_{D}(0) \Phi(0)\right\} & =\frac{1}{\sqrt{2 \pi \xi^{2}}} \int_{-\infty}^{\infty} \mathrm{d} \varphi \varphi \exp \left\{-\frac{1}{2 \xi^{2}}(\varphi-\phi)^{2}\right\}^{\varphi \rightarrow \underline{\varphi}+\phi} \phi,
\end{aligned}
$$

etc. Similarly, one finds since only Gaussian integrations appear that all initial $n$-point functions with $n>2$ are given in terms of the one- and two-point functions. Note also that the anti-symmetrized initial correlator involving the commutator of $\Phi$ and $\partial_{t} \Phi$ is not independent because of the field commutation relation.

The crucial observation is that higher initial time derivatives are not independent as can be observed from the exact field equation of motion, which reads for the real scalar $\Phi^{4}$-theory:

$$
\left\langle\partial_{t}^{2} \Phi\right\rangle=-m^{2}\langle\Phi\rangle-\frac{\lambda}{6 N}\left\langle\Phi^{3}\right\rangle .
$$

Since $\left\langle\Phi^{3}\right\rangle$ is given in terms of one- and two-point functions for Gaussian $\rho_{D}(0)$ also second and higher time derivatives are not independent. We conclude that the most general Gaussian density matrix is indeed described by the five parameters appearing in (97). In particular, all observable information contained in the density matrix can be conveniently expressed in terms of correlation functions and their derivatives (98) and (99).

For further interpretation of the initial conditions we note that

$$
\operatorname{Tr} \rho_{D}^{2}(0)=\int_{-\infty}^{\infty} \mathrm{d} \varphi \int_{-\infty}^{\infty} \mathrm{d} \varphi^{\prime}\left\langle\varphi\left|\rho_{D}(0)\right| \varphi^{\prime}\right\rangle\left\langle\varphi^{\prime}\left|\rho_{D}(0)\right| \varphi\right\rangle=\frac{1}{\sigma}
$$

The latter shows that for $\sigma>1$ the density matrix describes a mixed state. A pure state requires $\sigma=1$ or, equivalently, using (99) this condition can be expressed in terms of initial-time correlators as

$$
\begin{aligned}
{\left[\operatorname{Tr}\left\{\rho_{D}(0) \Phi(t) \Phi\left(t^{\prime}\right)\right\}_{\mid t=t^{\prime}=0}-\phi \phi\right]\left[\operatorname{Tr}\left\{\rho_{D}(0) \partial_{t} \Phi(t) \partial_{t^{\prime}} \Phi\left(t^{\prime}\right)\right\}_{\mid t=t^{\prime}=0}-\dot{\phi} \dot{\phi}\right] } \\
-\left[\frac{1}{2} \operatorname{Tr}\left\{\rho_{D}(0)\left(\partial_{t} \Phi(t) \Phi\left(t^{\prime}\right)+\Phi(t) \partial_{t^{\prime}} \Phi\left(t^{\prime}\right)\right)\right\}_{\mid t=t^{\prime}=0}-\dot{\phi} \phi\right]^{2}=\frac{1}{4}
\end{aligned}
$$


In field theory such a Gaussian initial condition is associated with a vanishing initial particle number, as will be discussed in Sec. 4. For $\sigma=1$ the "mixing term" in (97) is absent and one obtains a pure-state density matrix of the product form:

$$
\rho_{D}(0)=|\Omega\rangle\langle\Omega|
$$

with Schrödinger wave function

$$
\langle\varphi \mid \Omega\rangle=\frac{1}{\left(2 \pi \xi^{2}\right)^{1 / 4}} \exp \left\{i \dot{\phi} \varphi-\left(\frac{1}{4 \xi^{2}}+i \frac{\eta}{2 \xi}\right)(\varphi-\phi)^{2}\right\} .
$$

In order to go beyond Gaussian initial density matrices one can generalize the above example and parametrize the most general density matrix as

$$
\left\langle\varphi^{(1)}\left|\rho_{D}(0)\right| \varphi^{(2)}\right\rangle=\mathscr{N} e^{i f_{\mathscr{C}}[\varphi]},
$$

with normalization factor $\mathscr{N}$ and $f_{\mathscr{C}}[\varphi]$ expanded in powers of the fields:

$$
\begin{aligned}
f_{\mathscr{C}}[\varphi]= & \alpha_{0}+\int_{x} \alpha_{1}(x) \varphi(x)+\frac{1}{2} \int_{x y} \alpha_{2}(x, y) \varphi(x) \varphi(y)+\frac{1}{3 !} \int_{x y z} \alpha_{3}(x, y, z) \varphi(x) \varphi(y) \varphi(z) \\
& +\frac{1}{4 !} \int_{x y z w} \alpha_{4}(x, y, z, w) \varphi(x) \varphi(y) \varphi(z) \varphi(w)+\ldots
\end{aligned}
$$

Here $\varphi\left(0^{+}, \mathbf{x}\right)=\varphi^{(1)}(\mathbf{x})$ and $\varphi\left(0^{-}, \mathbf{x}\right)=\varphi^{(2)}(\mathbf{x})$. We emphasize that (108) employs a compact notation: since the density matrix $\rho_{D}(0)$ is specified at initial time $t_{0}=0$, all time integrals contribute only at time $t_{0}$ of the closed time contour. As a consequence, the coefficients $\alpha_{1}(x), \alpha_{2}(x, y), \alpha_{3}(x, y, z), \ldots$ vanish identically for times different than $t_{0}$. For instance, up to quadratic order one has

$$
\begin{aligned}
& \int_{x} \alpha_{1}(x) \varphi(x) \equiv \int_{\mathbf{x}}\left\{\alpha_{1}^{(1)}(\mathbf{x}) \varphi^{(1)}(\mathbf{x})+\alpha_{1}^{(2)}(\mathbf{x}) \varphi^{(2)}(\mathbf{x})\right\} \\
& \int_{x y} \alpha_{2}(x, y) \varphi(x) \varphi(y) \equiv \int_{\mathbf{x y}}\left\{\alpha_{2}^{(1,1)}(\mathbf{x}, \mathbf{y}) \varphi^{(1)}(\mathbf{x}) \varphi^{(1)}(\mathbf{y})+\alpha_{2}^{(1,2)}(\mathbf{x}, \mathbf{y}) \varphi^{(1)}(\mathbf{x}) \varphi^{(2)}(\mathbf{y})\right. \\
&\left.+\alpha_{2}^{(2,1)}(\mathbf{x}, \mathbf{y}) \varphi^{(2)}(\mathbf{x}) \varphi^{(1)}(\mathbf{y})+\alpha_{2}^{(2,2)}(\mathbf{x}, \mathbf{y}) \varphi^{(2)}(\mathbf{x}) \varphi^{(2)}(\mathbf{y})\right\} .
\end{aligned}
$$

Note that $\alpha_{0}$ is an irrelevant normalization constant. For a physical density matrix the other coefficients are of course not arbitrary. Hermiticity implies $\alpha_{1}^{(1)}=-\alpha_{1}^{(2) *}, \alpha_{2}^{(1,1)}=$ $-\alpha_{2}^{(2,2) *}$ and $\alpha_{2}^{(1,2)}=-\alpha_{2}^{(2,1) *}$, which can be directly compared to the discussion above.

\subsection{Nonequilibrium $2 \mathrm{PI}$ effective action}

Using the parametrization (107) and (108) for the most general initial density matrix, one observes that the generating functional (96) introduced above can be written as

$$
Z\left[J, R ; \rho_{D}\right]=\int \mathscr{D} \varphi e^{i\left(S[\varphi]+\int_{x} J(x) \varphi(x)+\frac{1}{2} \int_{x y} R(x, y) \varphi(x) \varphi(y)+\frac{1}{3 !} \int_{x y z} \alpha_{3}(x, y, z) \varphi(x) \varphi(y) \varphi(z)+\ldots\right)} .
$$


Here we have neglected an irrelevant normalization constant and rescaled the sources in (96) as $J(x) \rightarrow J(x)-\alpha_{1}(x)$ and $R(x, y) \rightarrow R(x, y)-\alpha_{2}(x, y)$. The sources can therefore be conveniently used to absorb the lower linear and quadratic contributions coming from the density matrix specifying the initial state. This absorbtion of $\alpha_{1}$ in $J$ and $\alpha_{2}$ in $R$ exploits the fact that the initial density matrix is completely encoded in terms of initialtime sources $\alpha_{1}, \alpha_{2}, \alpha_{3}, \ldots$ for the functional integral.

The generating functional (109) can be used to describe situations involving arbitrarily complex initial density matrices. However, often the initial conditions of an experiment may be described by only a few lowest $n$-point functions. For many practical purposes the initial density matrix is well described by a Gaussian one. For instance, the initial conditions for the reheating dynamics in the early universe at the end of inflation are described by a Gaussian density matrix to high accuracy. Clearly, the subsequent evolution builds up higher correlations which are crucial for the process of thermalization. These have to be taken into account by the quantum dynamics, which is not approximated by the specification of an initial density matrix!

From (109) one observes that for Gaussian initial density matrices, for which $\alpha_{3}=$ $\alpha_{4}=\ldots=0$, one has

$$
Z\left[J, R ; \rho_{D}^{\text {(gauss) }}\right] \equiv Z[J, R]
$$

As a consequence, in this case the nonequilibrium generating functional corresponds to the 2PI generating functional introduced in Eq. (14) for a closed time path. We can therefore directly take over all steps from Sec. 2 in order to construct the nonequilibrium 2PI effective action and obtain the important result:

$$
\text { Nonequilibrium 2PI effective action }=\Gamma[\phi, G] \text { with closed time path } \mathscr{C}
$$

All previous discussions of Sec. 2 remain unchanged except that for nonequilibrium the time integrals involve a closed time path! We emphasize again that the use of the nonequilibrium 2PI effective action represents no approximation for the dynamics higher irreducible correlations can build up corresponding to a non-Gaussian density matrix for times $t>t_{0}$. It only restricts the "experimental" setup described by the initial conditions for correlation functions. Non-Gaussian initial density matrices pose no problems in principle but require taking into account additional initial-time sources. This is most efficiently described in terms of $n$ PI effective actions for $n>2$.

\subsection{Exact evolution equations}

Without approximation the 2PI effective action $\Gamma[\phi, G]$ contains the complete information about the quantum theory. The functional representation of the nonequilibrium $\Gamma[\phi, G]$ employs a one-point $(\phi)$ and a two-point field $(G)$, whose physical values have to be computed for all times of interest. The equations of motion for these fields are given by the stationarity conditions (24) and (25).

We will consider first the scalar field theory in the symmetric regime, where $\Gamma[\phi=0, G] \equiv \Gamma[G]$ is sufficient. We come back to the case of a nonvanishing field expectation value as well as to fermionic and gauge fields below. The equation of 
motion (29) for $G$ reads

$$
G^{-1}(x, y)=G_{0}^{-1}(x, y)-\Sigma(x, y ; G)-i R(x, y),
$$

where the self-energy $\Sigma(x, y ; G)$ is given by (30) The form of the equation (111) is suitable for boundary value problems as e.g. appear for thermal equilibrium. However, nonequilibrium time evolution is an initial value problem. The equation of motion can be rewritten as a partial differential equation suitable for initial-value problems by convolution with $G$, using $\int_{z} G^{-1}(x, z) G(z, y)=\delta(x-y)$ :

$$
\int_{z} G_{0}^{-1}(x, z) G(z, y)-\int_{z}[\Sigma(x, z)+i R(x, y)] G(z, y)=\delta(x-y) .
$$

Here we employ the notation $\delta(x-y) \equiv \delta_{\mathscr{C}}\left(x^{0}-y^{0}\right) \delta(\mathbf{x}-\mathbf{y})$. For the scalar theory the classical propagator (cf. Sec. 2) is $G_{0}^{-1}(x-y)=i\left[\square_{x}+m^{2}\right] \delta(x-y)$ and one obtains the evolution equation for the time-ordered propagator:

$$
\left(\square_{x}+m^{2}\right) G(x, y)+i \int_{z}[\Sigma(x, z ; G)+i R(x, y)] G(z, y)=-i \delta(x-y)
$$

\subsubsection{Spectral and statistical components}

In the following we introduce a decomposition of the two-point function $G$ into spectral and statistical components. The corresponding evolution equations for the spectral function and statistical propagator are fully equivalent to the evolution equation for $G$, but have a simple physical interpretation. While the spectral function encodes the spectrum of the theory, the statistical propagator gives information about occupation numbers. Loosely speaking, the decomposition makes explicit what states are available and how often they are occupied.

For the real scalar field theory there are two independent real-valued two-point functions, which can be associated to the expectation value of the commutator and the anti-commutator of two fields,

$$
\begin{aligned}
\text { commutator: } & \rho(x, y)=i\langle[\Phi(x), \Phi(y)]\rangle, \\
\text { anti-commutator: } & F(x, y)=\frac{1}{2}\langle\{\Phi(x), \Phi(y)\}\rangle .
\end{aligned}
$$

Here $\rho(x, y)$ denotes the spectral function and $F(x, y)$ the statistical two-point function. The decomposition identity for spectral and statistical components of the propagator reads:

$$
G(x, y)=F(x, y)-\frac{i}{2} \rho(x, y) \operatorname{sign}_{\mathscr{C}}\left(x^{0}-y^{0}\right)
$$

The identity is easily understood by making the time-ordering for the propagator explicit:

$$
G(x, y)=\langle\Phi(x) \Phi(y)\rangle \Theta_{\mathscr{C}}\left(x^{0}-y^{0}\right)+\langle\Phi(y) \Phi(x)\rangle \Theta_{\mathscr{C}}\left(y^{0}-x^{0}\right)
$$




$$
\begin{aligned}
& =\frac{1}{2}\langle\{\Phi(x), \Phi(y)\}\rangle\left(\Theta_{\mathscr{C}}\left(x^{0}-y^{0}\right)+\Theta_{\mathscr{C}}\left(y^{0}-x^{0}\right)\right) \\
& -\frac{i}{2} i\langle[\Phi(x), \Phi(y)]\rangle \underbrace{\left(\Theta_{\mathscr{C}}\left(x^{0}-y^{0}\right)-\Theta_{\mathscr{C}}\left(y^{0}-x^{0}\right)\right)}_{\operatorname{sign}_{\mathscr{C}}\left(x^{0}-y^{0}\right) .}
\end{aligned}
$$

For real scalar fields the real functions obey $F(x, y)=F(y, x)$ and $\rho(x, y)=-\rho(y, x)$. We note from (114) that the spectral function $\rho$ encodes the equal-time commutation relations

$$
\left.\rho(x, y)\right|_{x^{0}=y^{0}}=0 \quad,\left.\quad \partial_{x^{0}} \rho(x, y)\right|_{x^{0}=y^{0}}=\delta(\mathbf{x}-\mathbf{y}) .
$$

The spectral function is also directly related to the retarded propagator $\rho(x, y) \Theta\left(x^{0}-y^{0}\right)$, or the advanced one $-\rho(x, y) \Theta\left(y^{0}-x^{0}\right)$. However, it is important to realize that out of equilibrium there are only two independent two-point functions - no more. These can be associated to $F$ and $\rho$, which has the advantage compared to e.g. an advanced propagator that the non-analyticity entering through time-ordering is always explicit.

To obtain a similar decomposition for the self-energy, we separate $\Sigma$ in a "local" and "nonlocal" part according to

$$
\Sigma(x, y ; G)=-i \Sigma^{(0)}(x ; G) \delta(x-y)+\bar{\Sigma}(x, y ; G) .
$$

Since $\Sigma^{(0)}$ just corresponds to a space-time dependent mass-shift it is convenient for the following to introduce the notation

$$
M^{2}(x ; G)=m^{2}+\Sigma^{(0)}(x ; G) .
$$

To make the time-ordering for the non-local part of the self-energy, $\bar{\Sigma}(x, y ; G)$, explicit we can use the same identity as for the propagator (116) to decompose:

$$
\bar{\Sigma}(x, y)=\Sigma_{F}(x, y)-\frac{i}{2} \Sigma_{\rho}(x, y) \operatorname{sign}_{\mathscr{C}}\left(x^{0}-y^{0}\right) .
$$

Though the discussion is given here for a vanishing field expectation value, it should be emphasized that the same decompositions (116) and (120) apply also for $\phi \neq 0$. The r.h.s. of (115) would then receive an additional contribution subtracting the disconnected part $\sim \phi \phi$. Equivalently, one can always view (116) as defining $F$ and $\rho$ from the connected propagator $G$ irrespective of the value of $\phi$.

We emphasize that the equivalent decomposition can be done for fermionic degrees of freedom as well. The fermion propagator in terms of spectral and statistical components reads in the same way as for bosons:

$$
\Delta(x, y)=F^{(f)}(x, y)-\frac{i}{2} \rho^{(f)}(x, y) \operatorname{sign}_{\mathscr{C}}\left(x^{0}-y^{0}\right) .
$$

However, in contrast to bosons for fermions the field anti-commutator corresponds to the spectral function,

$$
\begin{aligned}
\text { anti-commutator: } & \rho^{(f)}(x, y)=i\langle\{\Psi(x), \bar{\Psi}(y)\}\rangle, \\
\text { commutator: } & F^{(f)}(x, y)=\frac{1}{2}\langle[\Psi(x), \bar{\Psi}(y)]\rangle .
\end{aligned}
$$


This can be directly observed from the time-ordered fermion propagator $\Delta(x, y)=$ $\langle\Psi(x) \bar{\Psi}(y)\rangle \Theta_{\mathscr{C}}\left(x^{0}-y^{0}\right)-\langle\bar{\Psi}(y) \Psi(x)\rangle \Theta_{\mathscr{C}}\left(y^{0}-x^{0}\right)$. Here the minus sign is a consequence of the anti-commutation property for fermionic fields (cf. Sec. 2.3). In analogy to the bosonic case, the equal-time anti-commutation relations for the fields are again encoded in $\rho^{(f)}(x, y)$. For instance, for Dirac fermions one has

$$
\left.\gamma^{0} \rho(x, y)\right|_{x^{0}=y^{0}}=i \delta(\mathbf{x}-\mathbf{y})
$$

with the Dirac matrix $\gamma^{0}$, and the two-point functions have the hermiticity properties

$$
(\rho(y, x))^{\dagger}=-\gamma^{0} \rho(x, y) \gamma^{0}, \quad(F(y, x))^{\dagger}=\gamma^{0} F(x, y) \gamma^{0} .
$$

The corresponding decomposition for the fermion self-energy reads: ${ }^{6}$

$$
\Sigma^{(f)}(x, y)=\Sigma_{F}^{(f)}(x, y)-\frac{i}{2} \Sigma_{\rho}^{(f)}(x, y) \operatorname{sign}_{\mathscr{C}}\left(x^{0}-y^{0}\right) .
$$

\subsubsection{Detour: Thermal equilibrium}

To see the above decomposition in a probably more familiar context, we consider for a moment thermal equilibrium. This is done for illustrational purposes only, and we emphasize that the notion of an equilibrium temperature is nowhere implemented in nonequilibrium quantum field theory. If a nonequilibrium evolution approaches thermal equilibrium at late times then this a prediction of the theory and not put in by hand.

The 2PI effective action in thermal equilibrium is given by the same expression (28) if the closed time path is replaced by an imaginary path $\mathscr{C}=[0,-i \beta]$. Here $\beta$ denotes the inverse temperature. Since thermal equilibrium is translation invariant, the two-point functions depend only on relative coordinates and it is convenient to consider the Fourier transforms $F^{(\mathrm{eq})}(\omega, \mathbf{p})$ and $\rho^{(\mathrm{eq})}(\omega, \mathbf{p})$ with

$$
F^{(\mathrm{eq})}(x, y)=\int \frac{\mathrm{d} \omega \mathrm{d}^{d} p}{(2 \pi)^{d+1}} e^{-i \omega\left(x^{0}-y^{0}\right)+i \mathbf{p}(\mathbf{x}-\mathbf{y})} F^{(\mathrm{eq})}(\omega, \mathbf{p})
$$

and equivalently for the thermal spectral function.

The periodicity ("KMS") condition characterizing thermal equilibrium for the propagator in imaginary time is given by $\left.G(x, y)\right|_{x^{0}=0}=\left.G(x, y)\right|_{x^{0}=-i \beta}$. Employing the decomposition identity (116) for the propagator $G$, one can write the periodicity condition as

$$
F^{(\mathrm{eq})}(\omega, \mathbf{p})+\frac{i}{2} \rho^{(\mathrm{eq})}(\omega, \mathbf{p})=e^{-\beta \omega}\left(F^{(\mathrm{eq})}(\omega, \mathbf{p})-\frac{i}{2} \rho^{(\mathrm{eq})}(\omega, \mathbf{p})\right)
$$

\footnotetext{
6 If there is a local contribution to the proper self-energy, one separates in complete analogy to the scalar equation (118). The decomposition (126) is taken for the non-local part of the self-energy, while the local contribution gives rise to an effective space-time dependent fermion mass term.
} 
To see this note that $x^{0}=0$ comes first on the imaginary path $\mathscr{C}=[0,-i \beta]$, while $x^{0}=-i \beta$ comes latest such that $\operatorname{sign}_{\mathscr{C}}\left(x^{0}-y^{0}\right)$ contributes opposite signs on the left and on the right of the equation. Eq. (128) can be rewritten in a more standard form as a fluctuation-dissipation relation for bosons: ${ }^{7}$

$$
F^{(\mathrm{eq})}(\omega, \mathbf{p})=-i\left(\frac{1}{2}+n_{\mathrm{BE}}(\omega)\right) \rho^{(\mathrm{eq})}(\omega, \mathbf{p})
$$

with $n_{\mathrm{BE}}(\omega)=\left(e^{\beta \omega}-1\right)^{-1}$ denoting the Bose-Einstein distribution function. Eq. (129) relates the spectral function to the statistical propagator. While $\rho^{(\mathrm{eq})}$ encodes the information about the spectrum of the theory, one observes from (129) that the function $F^{(e q)}$ encodes the statistical aspects in terms of the particle distribution function $n_{\mathrm{BE}}$. In the same way one obtains for the Fourier transforms of the spectral and statistical components of the self-energy the thermal equilibrium relation

$$
\Sigma_{F}^{(\mathrm{eq})}(\omega, \mathbf{p})=-i\left(\frac{1}{2}+n_{\mathrm{BE}}(\omega)\right) \Sigma_{\rho}^{(\mathrm{eq})}(\omega, \mathbf{p}) .
$$

We note that the ratio $\Sigma_{\rho}^{(\mathrm{eq})}(\omega, \mathbf{p}) / 2 \omega$ plays in the limit of a vanishing $\omega$-dependence the role of the decay rate for one-particle excited states with momentum $\mathbf{p}$.

For fermions the anti-periodicity condition of the fermionic propagator in thermal equilibrium, $\left.\Delta(x, y)\right|_{x^{0}=0}=-\left.\Delta(x, y)\right|_{x^{0}=-i \beta}$, implies a corresponding fluctuationdissipation relation. The difference is that the Bose-Einstein distribution in (129) is replaced by the Fermi-Dirac distribution $n_{\mathrm{FD}}(\omega)=\left(e^{\beta \omega}+1\right)^{-1}$ according to $1 / 2+$ $n_{\mathrm{BE}}(\omega) \rightarrow 1 / 2-n_{\mathrm{FD}}(\omega)$ in the respective relation.

It is important to realize that out of equilibrium $F$ and $\rho$ are not related by the fluctuation-dissipation relation! In contrast to the nonequilibrium theory, the relation (129) is a manifestation of the tremendous simplification that happens if the system is in thermal equilibrium. An even more stringent reduction occurs for the vacuum where $n_{\mathrm{BE}}(\omega) \equiv 0$. In this respect, nonequilibrium quantum field theory is more complicated since it admits the description of more general situations. Of course, the nonequilibrium theory encompasses the thermal equilibrium or vacuum theory as special cases. We leave now this equilibrium detour and return to the nonequilibrium case.

\subsubsection{Nonequilibrium evolution equations}

Out of equilibrium we have to follow the time-evolution both for the statistical propagator, $F$, as well as for the spectral function, $\rho$. The evolution equations are obtained from (113) with the help of the identities (116) and (120). Most importantly, once expressed in terms of $F$ and $\rho$ the time-ordering is explicit and the respective sign-

\footnotetext{
${ }^{7}$ In our conventions the Fourier transform of the real-valued antisymmetric function $\rho(x, y)$ is purely imaginary.
} 
functions appearing in the time-ordered propagator can be conveniently evaluated along the time contour $\mathscr{C}$.

With the notation (118) the time evolution equation for the time-ordered propagator (113) reads

$$
\left[\square_{x}+M^{2}(x ; G)\right] G(x, y)+i \int_{z} \bar{\Sigma}(x, z ; G) G(z, y)=-i \delta(x-y),
$$

where we have set $R \equiv 0$. The influence of the initial-time sources encoded in $R$ is discussed below. For the evaluation along the time contour $\mathscr{C}$ involved in the integration with $\int_{z} \equiv \int_{\mathscr{C}} \mathrm{d} z^{0} \int \mathrm{d}^{d} z$ we employ (116) and (120):

$$
\begin{aligned}
& i \int_{z} \bar{\Sigma}(x, z ; G) G(z, y)=i \int_{z}\left\{\Sigma^{F}(x, z) F(z, y)\right. \\
& -\frac{i}{2} \Sigma^{F}(x, z) \rho(z, y) \operatorname{sign}_{\mathscr{C}}\left(z^{0}-y^{0}\right)-\frac{i}{2} \Sigma^{\rho}(x, z) F(z, y) \operatorname{sign}_{\mathscr{C}}\left(x^{0}-z^{0}\right) \\
& \left.-\frac{1}{4} \Sigma^{\rho}(x, z) \rho(z, y) \operatorname{sign}_{\mathscr{C}}\left(x^{0}-z^{0}\right) \operatorname{sign}_{\mathscr{C}}\left(z^{0}-y^{0}\right)\right\} .
\end{aligned}
$$

The first term on the r.h.s. vanishes because of integration along the closed time contour $\mathscr{C}$ (cf. Sec. 3.1). To proceed for the second term one splits the contour integral such that the sign-functions have a definite value, for instance

$$
\int_{\mathscr{C}} \mathrm{d} z^{0} \operatorname{sign}_{\mathscr{C}}\left(z^{0}-y^{0}\right)=\int_{0}^{y^{0}} \mathrm{~d} z^{0}(-1)+\int_{y^{0}}^{0} \mathrm{~d} z^{0}=-2 \int_{0}^{y^{0}} \mathrm{~d} z^{0}
$$

for the closed contour with initial time $t_{0}=0$. To evaluate the last term on the r.h.s. of Eq. (132) it is convenient to distinguish the cases

(a) $\Theta_{\mathscr{C}}\left(x^{0}-y^{0}\right)=1$ :

$$
\int_{\mathscr{C}} \mathrm{d} z^{0} \operatorname{sign}_{\mathscr{C}}\left(x^{0}-z^{0}\right) \operatorname{sign}_{\mathscr{C}}\left(z^{0}-y^{0}\right)=\int_{0}^{y^{0}} \mathrm{~d} z^{0}(-1)+\int_{y^{0}}^{x^{0}} \mathrm{~d} z^{0}+\int_{x^{0}}^{0} \mathrm{~d} z^{0}(-1),
$$

(b) $\underline{\Theta_{\mathscr{C}}\left(y^{0}-x^{0}\right)=1}$ :

$$
\int_{\mathscr{C}} \mathrm{d} z^{0} \operatorname{sign}_{\mathscr{C}}\left(x^{0}-z^{0}\right) \operatorname{sign}_{\mathscr{C}}\left(z^{0}-y^{0}\right)=\int_{0}^{x^{0}} \mathrm{~d} z^{0}(-1)+\int_{x^{0}}^{y^{0}} \mathrm{~d} z^{0}+\int_{y^{0}}^{0} \mathrm{~d} z^{0}(-1) .
$$

One observes that (a) and (b) differ only by an overall sign factor $\sim \operatorname{sign}_{\mathscr{C}}\left(x^{0}-y^{0}\right)$. Combining the integrals therefore gives:

$$
\begin{aligned}
i \int_{z} \bar{\Sigma}(x, z ; G) G(z, y)= & \int \mathrm{d}^{d} z\left\{\int_{0}^{x^{0}} \mathrm{~d} z^{0} \Sigma_{\rho}(x, z) F(z, y)-\int_{0}^{y^{0}} \mathrm{~d} z^{0} \Sigma_{F}(x, z) \rho(z, y)\right. \\
& \left.-\frac{i}{2} \operatorname{sign}_{\mathscr{C}}\left(x^{0}-y^{0}\right) \int_{y^{0}}^{x^{0}} \mathrm{~d} z^{0} \Sigma_{\rho}(x, z) \rho(z, y)\right\} .
\end{aligned}
$$


One finally employs

$$
\square_{x} G(x, y)=\square_{x} F(x, y)-\frac{i}{2} \operatorname{sign}_{\mathscr{C}}\left(x^{0}-y^{0}\right) \square_{x} \rho(x, y)-i \delta(x-y)
$$

such that the $\delta$-term cancels with the respective one on the r.h.s. of the evolution equation (131). Here we have used

$$
\begin{aligned}
-\frac{i}{2} \partial_{x^{0}}^{2}\left[\rho(x, y) \operatorname{sign}_{\mathscr{C}}\left(x^{0}-y^{0}\right)\right]= & -\frac{i}{2} \operatorname{sign}_{\mathscr{C}}\left(x^{0}-y^{0}\right) \partial_{x^{0}}^{2} \rho(x, y) \\
& \underbrace{-i \delta_{\mathscr{C}}\left(x^{0}-y^{0}\right) \partial_{x^{0}} \rho(x, y)}_{-i \delta(x-y)}
\end{aligned}
$$

where (117) is employed for the last line and to observe that a term $\sim \rho(x, y) \delta_{\mathscr{C}}\left(x^{0}-y^{0}\right)$ vanishes identically. Comparing coefficients, which here corresponds to separating real and imaginary parts, one finds from (136) and (137) the equations for $F(x, y)$ and $\rho(x, y)$. Using the abbreviated notation $\int_{t_{1}}^{t_{2}} \mathrm{~d} z \equiv \int_{t_{1}}^{t_{2}} \mathrm{~d} z^{0} \int_{-\infty}^{\infty} \mathrm{d} d z$ we arrive at the coupled evolution equations for the spectral function and the statistical propagator:

$$
\begin{aligned}
& {\left[\square_{x}+M^{2}(x)\right] \rho(x, y)=-\int_{y^{0}}^{x^{0}} \mathrm{~d} z \Sigma_{\rho}(x, z) \rho(z, y),} \\
& {\left[\square_{x}+M^{2}(x)\right] F(x, y)=-\int_{0}^{x^{0}} \mathrm{~d} z \Sigma_{\rho}(x, z) F(z, y)+\int_{0}^{y^{0}} \mathrm{~d} z \Sigma_{F}(x, z) \rho(z, y) .}
\end{aligned}
$$

These are causal equations with characteristic "memory" integrals, which integrate over the time history of the evolution. We emphasize that the presence of memory integrals is a property of the exact theory and in accordance with all symmetries, in particular time reflection symmetry. The equations themselves do not single out a direction of time and they should be clearly distinguished from phenomenological nonequilibrium equations, where irreversibility is typically put in by hand. Since these equations are exact they are fully equivalent to any kind of identity for the two-point functions such as Schwinger-Dyson/Kadanoff-Baym equations without further approximations. For $\phi=0$ the functional dependence of the self-energy corrections in (138) is given by $M^{2}=M^{2}(F), \Sigma_{F}=\Sigma_{F}(\rho, F)$ and $\Sigma_{\rho}=\Sigma_{\rho}(\rho, F)$. The case $\phi \neq 0$ is discussed below.

Note that the initial-time properties of the spectral function have to comply with the equal-time commutation relations (117). In contrast, for $F(x, y)$ as well as its first derivatives the full initial conditions at $t_{0}=0$ need to be supplied in order to solve these equations. To make contact with the discussion of initial conditions in Sec. 3.2 and Eq. (99), we consider for a moment the spatially homogeneous case for which $F(x, y)=F\left(x^{0}, y^{0} ; \mathbf{x}-\mathbf{y}\right)=\int\left[\mathrm{d}^{d} p /(2 \pi)^{d}\right] \exp [i \mathbf{p}(\mathbf{x}-\mathbf{y})] F\left(x^{0}, y^{0} ; \mathbf{p}\right)$ and equivalently for $\rho(x, y)$. In terms of the Fourier components $F\left(t, t^{\prime} ; \mathbf{p}\right)$ the solution of the integrodifferential equations (138) requires the following initial conditions:

$$
F\left(t, t^{\prime} ; \mathbf{p}\right)_{\mid t=t^{\prime}=0} \equiv \xi_{\mathbf{p}}^{2} \quad, \quad \frac{1}{2}\left(\partial_{t} F\left(t, t^{\prime} ; \mathbf{p}\right)+\partial_{t^{\prime}} F\left(t, t^{\prime} ; \mathbf{p}\right)\right)_{\mid t=t^{\prime}=0} \equiv \xi_{\mathbf{p}} \eta_{\mathbf{p}}
$$




$$
\partial_{t} \partial_{t^{\prime}} F\left(t, t^{\prime} ; \mathbf{p}\right)_{\mid t=t^{\prime}=0} \equiv \eta_{\mathbf{p}}^{2}+\frac{\sigma_{\mathbf{p}}^{2}}{4 \xi_{\mathbf{p}}^{2}} .
$$

Here we have used that the required correlators at initial time are identical to those given in Eq. (99) for the considered case $\phi \equiv 0$, where we had suppressed the momentum labels in the notation. Accordingly, these are the very same parameters that have to be specified for the corresponding most general Gaussian initial density matrix (97). We emphasize that the initial conditions for the spectral function equation are completely fixed by the properties of the theory itself: the equal-time commutation relations (117) specify $\left.\rho\left(t, t^{\prime} ; \mathbf{p}\right)\right|_{t=t^{\prime}=0}=0,\left.\partial_{t} \rho\left(t, t^{\prime} ; \mathbf{p}\right)\right|_{t=t^{\prime}=0}=1$ and $\left.\partial_{t} \partial_{t^{\prime}} \rho\left(t, t^{\prime} ; \mathbf{p}\right)\right|_{t=t^{\prime}=0}=0$ for the anti-symmetric spectral function.

We are now in the position to discuss the role of the initial-time sources, which contain the information about the initial-time density matrix. According to the discussion of Secs. 3.2 and 3.3 the initial-time sources are fully described by the Fourier components of the bilinear source term $R\left(t, t^{\prime} ; \mathbf{p}\right)$ at $t=t^{\prime}=t_{0}=0$ for the case $\phi=0$. Mathematically, the role of the initial-time sources for the evolution equations is rather simple: Since these sources have support only for the initial time $t_{0}$ and vanish identically for times $t \neq t_{0}$, they only fix the initial values for the correlators and their first derivatives. ${ }^{8}$ For simpler differential equations this property is well documented in the literature on the theory of Green's functions. In order to see that this indeed holds also for the case considered here, recall that the time evolution equations (138) are derived from (113) for $R \equiv 0$. Eq. (139) shows that there is a one-to-one correspondence between the initialtime sources parametrizing the density matrix (97) and the required initial conditions for the solution of the time evolution equations. To check that no further dependencies on $R$ remain in the evolution equations for times $t \neq t_{0}$, one notes that the $R$-dependence appears in (113) as a term $\sim \int_{z} R(x, z) G(z, y)$, which identically vanishes for $x^{0} \neq t_{0}$. As a consequence, the equation governing $G(x, y)$ (or $F$ and $\rho$ ) cannot explicitly depend on $R$ for its time arguments different than $t_{0}$, i.e. for all times of interest.

The clear separation of the dynamical role of spectral and statistical components is a generic property of nonequilibrium field theory. As discussed in Sec. 3.4.2, the nonequilibrium theory encompasses standard vacuum theory as a special case where this separation is absent. This dichotomy for nonequilibrium time evolution equations is not specific to scalar field degrees of freedom. In terms of spectral and statistical components the equations for fermionic fields or gauge fields have a very similar structure as well. For instance, the respective form of the evolution equations for the fermion spectral function $\rho^{(f)}(x, y)$ and statistical propagator $F^{(f)}(x, y)$ defined in (121) can be directly obtained from (138) by the 1.h.s. replacement:

$$
\left[\square_{x}+M^{2}\right] \rho(x, y) \longrightarrow-\left[i \not_{x}-m^{(f)}\right] \rho^{(f)}(x, y)
$$

and equivalently for $F^{(f)}(x, y)$. On the r.h.s. of (138) then appear the respective fermion propagators and self-energies $\Sigma_{\rho}^{(f)}$ and $\Sigma_{F}^{(f)}$ as defined in (126). This can be directly verified from the equation of motion for the time-ordered fermion propagator (66) by

${ }^{8}$ We consider additional external sources to be absent. 
convoluting it with $\Delta$. For a free inverse propagator as in (71) for Dirac fermions this yields

$$
\left[i \partial_{x}-m^{(f)}\right] \Delta(x, y)-i \int_{z} \Sigma^{(f)}(x, z) \Delta(z, y)=i \delta_{\mathscr{C}}(x-y) .
$$

Following along the lines of the above discussion for scalars one finds for the fermion case the coupled evolution equations:

$$
\begin{aligned}
{\left[i \not \partial_{x}-m^{(f)}\right] \rho^{(f)}(x, y) } & =\int_{y^{0}}^{x^{0}} \mathrm{~d} z \Sigma_{\rho}^{(f)}(x, z) \rho^{(f)}(z, y), \\
{\left[i \not \partial_{x}-m^{(f)}\right] F^{(f)}(x, y) } & =\int_{0}^{x^{0}} \mathrm{~d} z \Sigma_{\rho}^{(f)}(x, z) F^{(f)}(z, y)-\int_{0}^{y^{0}} \mathrm{~d} z \Sigma_{F}^{(f)}(x, z) \rho^{(f)}(z, y) .
\end{aligned}
$$

Similarly, the nonequilibrium evolution equations for gauge fields can be obtained as well. For instance, denoting the full gauge field propagator by

$$
D^{\mu v}(x, y)=F_{D}^{\mu v}(x, y)-\frac{i}{2} \rho_{D}^{\mu v}(x, y) \operatorname{sign}_{\mathscr{C}}\left(x^{0}-y^{0}\right)
$$

for a theory with free inverse gauge propagator given by

$$
i D_{0, \mu \nu}^{-1}(x, y)=\left[g_{\mu \nu} \square-\left(1-\xi^{-1}\right) \partial_{\mu} \partial_{\nu}\right]_{x} \delta(x-y)
$$

for covariant gauges with gauge-fixing parameter $\xi$ and vanishing "background" fields, one finds the respective equations from (138) by

$$
\left[\square_{x}+M^{2}\right] \rho(x, y) \longrightarrow-\left[g^{\mu} \square-\left(1-\xi^{-1}\right) \partial^{\mu} \partial_{\gamma}\right]_{x} \rho_{D}^{\gamma v}(x, y)
$$

and equivalently for $F_{D}^{\gamma v}(x, y)$. Of course, the respective indices have to be attached to the corresponding self-energies on the r.h.s. of the equations. The derivation of the nonequilibrium gauge field evolution equations will be discussed in more detail in Sec. 6 in the context of higher $n$ PI effective actions with $n>2$.

\subsubsection{Non-zero field expectation value}

In the presence of a non-zero field expectation value, $\phi \neq 0$, the form of the scalar evolution equations for the spectral and statistical function (138) remain the same. However, the functional dependence now includes $M^{2}=M^{2}(\phi, F), \Sigma_{F}=\Sigma_{F}(\phi, \rho, F)$ and $\Sigma_{\rho}=\Sigma_{\rho}(\phi, \rho, F)$. Note that the local self-energy correction (119) described by $M^{2}$ does not depend on the spectral function because the latter vanishes for equal-time arguments. For the $N$-component scalar field theory (13) one has with $\phi^{2} \equiv \phi_{a} \phi_{a}$ :

$$
\begin{aligned}
M_{a b}^{2}(x ; \phi, F) & =\left(m^{2}+\frac{\lambda}{6 N}\left[F_{c c}(x, x)+\phi^{2}(x)\right]\right) \delta_{a b} \\
& +\frac{\lambda}{3 N}\left[F_{a b}(x, x)+\phi_{a}(x) \phi_{b}(x)\right]
\end{aligned}
$$


with the respective field indices attached to (138). In this case the evolution equations for the spectral function and statistical two-point function (138) are supplemented by a differential equation for $\phi$ given by the stationarity condition (24), which yields the field evolution equation:

$$
\left(\left[\square_{x}+\frac{\lambda}{6 N} \phi^{2}(x)\right] \delta_{a b}+M_{a b}^{2}(x ; \phi \equiv 0, F)\right) \phi_{b}(x)=\frac{\delta \Gamma_{2}}{\delta \phi_{a}(x)}
$$

For comparison the corresponding equation for the classical field theory, $\delta S[\phi] / \delta \phi_{a}(x)$, is obtained from (147) by the replacement $M_{a b}(x ; \phi \equiv 0, F) \rightarrow m^{2} \delta_{a b}$ and $\Gamma_{2} \equiv 0$. For the above field evolution equation we have used that the one-loop type contribution to the 2 PI effective action reads

$$
\frac{i}{2} \operatorname{Tr} G_{0}^{-1}(\phi) G=-\frac{1}{2} \int_{x}\left(\left[\square_{x}+m^{2}+\frac{\lambda}{6 N} \phi^{2}(x)\right] \delta_{a b}+\frac{\lambda}{3 N} \phi_{a}(x) \phi_{b}(x)\right) F_{b a}(x, x)
$$

for the classical inverse propagator (20), such that one can write

$$
m^{2}-\frac{\delta\left[i \operatorname{Tr} G_{0}^{-1}(\phi) G / 2\right]}{\delta \phi_{a}(x)}=M_{a b}^{2}(x ; \phi \equiv 0, F) .
$$

The solution of (147) requires specifying the field and its first derivative at initial time. In the context of the above discussion for homogeneous fields this just corresponds to specifying (98), which together with the required initial conditions (139) for the twopoint function completes the correspondence with the initial-time density matrix (97).

\subsubsection{Lorentz decomposition for fermion dynamics}

The evolution equations for the fermion spectral function and statistical propagator (142) are rather complicated in general. For Dirac fermions each two-point function contains 16 complex components, which is often supplemented by additional field attributes such as "flavor" or "color". However, it is often not necessary in practice to consider all components due to the presence of symmetries, which require certain components to vanish identically without loss of generality. Depending on the symmetry properties of the initial state and interaction these terms remain zero under the nonequilibrium time evolution, which can dramatically simplify the analysis. It is typically very useful to decompose the fields $\rho^{(f)}(x, y)$ and $F^{(f)}(x, y)$ of Eq. (142) into terms that have definite transformation properties under Lorentz transformation. In order to ease the notation, in this section we will write $\rho^{(f)} \mapsto \rho, F^{(f)} \mapsto F, \Sigma_{\rho}^{(f)} \mapsto A$ and $\Sigma_{F}^{(f)} \mapsto C$. Using a standard basis we write

$$
\rho=\rho_{S}+i \gamma_{5} \rho_{P}+\gamma_{\mu} \rho_{V}^{\mu}+\gamma_{\mu} \gamma_{5} \rho_{A}^{\mu}+\frac{1}{2} \sigma_{\mu v} \rho_{T}^{\mu v}
$$

where $\sigma_{\mu \nu}=\frac{i}{2}\left[\gamma_{\mu}, \gamma_{v}\right]$ and $\gamma_{5}=i \gamma^{0} \gamma^{1} \gamma^{2} \gamma^{3}$. The 16 (pseudo-)scalar, (pseudo-)vector and tensor components

$$
\rho_{S}=\tilde{\operatorname{tr}} \rho, \quad \rho_{P}=-i \tilde{\operatorname{tr}} \gamma_{5} \rho, \quad \rho_{V}^{\mu}=\tilde{\operatorname{tr}} \gamma^{\mu} \rho, \quad \rho_{A}^{\mu}=\tilde{\operatorname{tr}} \gamma_{5} \gamma^{\mu} \rho, \quad \rho_{T}^{\mu v}=\tilde{\operatorname{tr}} \sigma^{\mu v} \rho,
$$


are complex two-point functions. Here we have defined $\tilde{\mathrm{tr}} \equiv \frac{1}{4} \operatorname{tr}$ where the trace acts in Dirac space. Equivalently, there are 16 complex components for $F, A$ and $C$ for given other field attributes. Using the hermiticity properties (125) they obey

$$
\rho_{i j}^{(\Gamma)}(x, y)=-\left(\rho_{j i}^{(\Gamma)}(y, x)\right)^{*} \quad, \quad F_{i j}^{(\Gamma)}(x, y)=\left(F_{j i}^{(\Gamma)}(y, x)\right)^{*}
$$

where $\Gamma=\{S, P, V, A, T\}$. Inserting the above decomposition into the evolution equations (142) one obtains the respective equations for the various components displayed in (151).

For a more detailed discussion, we first consider the 1.h.s. of the evolution equations (142). In fact, the approximation of a vanishing r.h.s. corresponds to the standard meanfield or Hartree-type approaches frequently discussed in the literature. However, to discuss thermalization we have to go beyond such a "Gaussian" approximation: it is crucial to include direct scattering which is described by the nonvanishing contributions from the r.h.s. of the evolution equations. This is discussed in detail in Sec. 4. Starting with the 1.h.s. of (142) for the evolution equation for the spectral function one finds:

$$
\begin{aligned}
\tilde{\operatorname{tr}}\left[\left(i \not \partial-m_{f}\right) \rho\right] & =\left(i \partial_{\mu} \rho_{V}^{\mu}\right)-m_{f} \rho_{S}, \\
-i \tilde{\operatorname{tr}}\left[\gamma_{5}\left(i \not \partial-m_{f}\right) \rho\right] & =-i\left(i \partial_{\mu} \rho_{A}^{\mu}\right)-m_{f} \rho_{P}, \\
\tilde{\operatorname{tr}}\left[\gamma^{\mu}\left(i \not \partial-m_{f}\right) \rho\right] & =\left(i \partial^{\mu} \rho_{S}\right)+i\left(i \partial_{v} \rho_{T}^{v \mu}\right)-m_{f} \rho_{V}^{\mu}, \\
\tilde{\operatorname{tr}}\left[\gamma_{5} \gamma^{\mu}\left(i \not \partial-m_{f}\right) \rho\right] & =i\left(i \partial^{\mu} \rho_{P}\right)+\frac{1}{2} \varepsilon^{\mu v \gamma \delta}\left(i \partial_{v} \rho_{T, \gamma \delta}\right)-m_{f} \rho_{A}^{\mu}, \\
\tilde{\operatorname{tr}}\left[\sigma^{\mu v}\left(i \not \partial-m_{f}\right) \rho\right] & =-i\left(i \partial^{\mu} \rho_{V}^{v}-i \partial^{v} \rho_{V}^{\mu}\right)+\varepsilon^{\mu v \gamma \delta}\left(i \partial_{\gamma} \rho_{A, \delta}\right)-m_{f} \rho_{T}^{\mu v} .
\end{aligned}
$$

The corresponding expressions for the 1.h.s. of the evolution equation for the statistical propagator $F$ follow from (153) with the replacement $\rho \rightarrow F$. We turn now to the integrand on the r.h.s. of (142) for the evolution equation of $\rho$. For the various components (151) one finds:

$$
\begin{aligned}
\tilde{\operatorname{tr}}[A \rho]= & A_{S} \rho_{S}-A_{P} \rho_{P}+A_{V}^{\mu} \rho_{V, \mu}-A_{A}^{\mu} \rho_{A, \mu} \\
& +\frac{1}{2} A_{T}^{\mu v} \rho_{T, \mu v} \\
-i \tilde{\operatorname{tr}}\left[\gamma_{5} A \rho\right]= & A_{S} \rho_{P}+A_{P} \rho_{S}-i A_{V}^{\mu} \rho_{A, \mu}+i A_{A}^{\mu} \rho_{V, \mu} \\
& +\frac{1}{4} \varepsilon^{\mu v \gamma \delta} A_{T, \mu v} \rho_{T, \gamma \delta}, \\
\tilde{\operatorname{tr}}\left[\gamma^{\mu} A \rho\right]= & A_{S} \rho_{V}^{\mu}+A_{V}^{\mu} \rho_{S}-i A_{P} \rho_{A}^{\mu}+i A_{A}^{\mu} \rho_{P}+i A_{V, v} \rho_{T}^{v \mu} \\
& +i A_{T}^{\mu v} \rho_{V, v}+\frac{1}{2} \varepsilon^{\mu v \gamma \delta}\left(A_{A, v} \rho_{T, \gamma \delta}+A_{T, v \gamma} \rho_{A, \delta}\right), \\
\tilde{\operatorname{tr}}\left[\gamma_{5} \gamma^{\mu} A \rho\right]= & A_{S} \rho_{A}^{\mu}+A_{A}^{\mu} \rho_{S}-i A_{P} \rho_{V}^{\mu}+i A_{V}^{\mu} \rho_{P}+i A_{A, v} \rho_{T}^{v \mu} \\
& +i A_{T}^{\mu v} \rho_{A, v}+\frac{1}{2} \varepsilon^{\mu v \gamma \delta}\left(A_{V, v} \rho_{T, \gamma \delta}+A_{T, v \gamma} \rho_{V, \delta}\right),
\end{aligned}
$$




$$
\begin{aligned}
\tilde{\operatorname{tr}}\left[\sigma^{\mu v} A \rho\right]= & A_{S} \rho_{T}^{\mu v}+A_{T}^{\mu v} \rho_{S}-\frac{1}{2} \varepsilon^{\mu v \gamma \delta}\left(A_{P} \rho_{T, \gamma \delta}+A_{T, \gamma \delta} \rho_{P}\right) \\
& -i\left(A_{V}^{\mu} \rho_{V}^{v}-A_{V}^{v} \rho_{V}^{\mu}\right)+\varepsilon^{\mu v \gamma \delta}\left(A_{V, \gamma} \rho_{A, \delta}-A_{A, \gamma} \rho_{V, \delta}\right) \\
& +i\left(A_{A}^{\mu} \rho_{A}^{v}-A_{A}^{v} \rho_{A}^{\mu}\right)+i\left(A_{T}^{\mu \gamma} \rho_{T, \gamma}{ }^{v}-A_{T}^{v \gamma} \rho_{T, \gamma}{ }^{\mu}\right) .
\end{aligned}
$$

With the above expressions one obtains the evolution equations for the various Lorentz components in a straightforward way using (142). We note that the convolutions appearing on the r.h.s. of the evolution equation (142) for $F$ are of the same form than those computed above for $\rho$. The respective r.h.s. can be read off Eqs. (154)-(158) by replacing $\rho \rightarrow F$ for the first term and $A \rightarrow C$ for the second term under the integrals of Eq. (142) for $F$. We have now all the relevant building blocks to discuss the most general case of nonequilibrium fermionic fields. However, this is often not necessary in practice due to the presence of symmetries, which require certain components to vanish identically.

In the following, we will exploit symmetries of the action (68) for the chiral quarkmeson model described in Sec. 2.3.1 and of initial conditions in order to simplify the fermionic evolution equations:

Spatial translation invariance and isotropy: We will consider spatially homogeneous and isotropic initial conditions. In this case it is convenient to work in Fourier space and we write

$$
\rho(x, y) \equiv \rho\left(x^{0}, y^{0} ; \mathbf{x}-\mathbf{y}\right)=\int \frac{\mathrm{d}^{3} p}{(2 \pi)^{3}} e^{i \mathbf{p} \cdot(\mathbf{x}-\mathbf{y})} \rho\left(x^{0}, y^{0} ; \mathbf{p}\right),
$$

and similarly for the other two-point functions. Moreover, isotropy implies a reduction of the number of independent two-point functions: e.g. the vector components of the spectral function can be written as

$$
\rho_{V}^{0}\left(x^{0}, y^{0} ; \mathbf{p}\right)=\rho_{V}^{0}\left(x^{0}, y^{0} ; p\right), \quad \vec{\rho}_{V}\left(x^{0}, y^{0} ; \mathbf{p}\right)=\mathbf{v} \rho_{V}\left(x^{0}, y^{0} ; p\right),
$$

where $p \equiv|\mathbf{p}|$ and $\mathbf{v}=\mathbf{p} / p$.

Parity: The vector components $\rho_{V}^{0}\left(x^{0}, y^{0} ; p\right)$ and $\rho_{V}\left(x^{0}, y^{0} ; p\right)$ are unchanged under a parity transformation, whereas the corresponding axial-vector components get a minus sign. Therefore, parity together with rotational invariance imply that

$$
\rho_{A}^{0}\left(x^{0}, y^{0} ; p\right)=\rho_{A}\left(x^{0}, y^{0} ; p\right)=0 .
$$

The same is true for the axial-vector components of $F, A$ and $C$. Parity also implies the pseudo-scalar components of the various two-point functions to vanish.

$C P$-invariance: For instance, under combined charge conjugation and parity transformation the vector component of $\rho$ transforms as

$$
\begin{aligned}
& \rho_{V}^{0}\left(x^{0}, y^{0} ; p\right) \longrightarrow \rho_{V}^{0}\left(y^{0}, x^{0} ; p\right), \\
& \rho_{V}\left(x^{0}, y^{0} ; p\right) \longrightarrow-\rho_{V}\left(y^{0}, x^{0} ; p\right),
\end{aligned}
$$

and similarly for $A_{V}^{0}$ and $A_{V}$. The $F$-components transform as

$$
F_{V}^{0}\left(x^{0}, y^{0} ; p\right) \longrightarrow-F_{V}^{0}\left(y^{0}, x^{0} ; p\right),
$$




$$
F_{V}\left(x^{0}, y^{0} ; p\right) \longrightarrow F_{V}\left(y^{0}, x^{0} ; p\right),
$$

and similarly for $C_{V}^{0}$ and $C_{V}$. Combining this with the hermiticity relations (152), one obtains for these components that

$$
\begin{aligned}
& \operatorname{Re} \rho_{V}^{0}\left(x^{0}, y^{0} ; p\right)=\mathfrak{I} \rho_{V}\left(x^{0}, y^{0} ; p\right)=0, \\
& \operatorname{Re} F_{V}^{0}\left(x^{0}, y^{0} ; p\right)=\mathfrak{I} F_{V}\left(x^{0}, y^{0} ; p\right)=0, \\
& \operatorname{Re} A_{V}^{0}\left(x^{0}, y^{0} ; p\right)=\mathfrak{I} A_{V}\left(x^{0}, y^{0} ; p\right)=0, \\
& \operatorname{Re} C_{V}^{0}\left(x^{0}, y^{0} ; p\right)=\mathfrak{I} C_{V}\left(x^{0}, y^{0} ; p\right)=0,
\end{aligned}
$$

for all times $x^{0}$ and $y^{0}$ and all individual momentum modes.

Chiral symmetry: The only components of the decomposition (150) allowed by chiral symmetry are those which anticommute with $\gamma_{5}$. In particular, chiral symmetry forbids a mass term for fermions $\left(m_{f} \equiv 0\right)$ and we have

$$
\rho_{S}\left(x^{0}, y^{0} ; \mathbf{p}\right)=\rho_{P}\left(x^{0}, y^{0} ; \mathbf{p}\right)=\rho_{T}^{\mu v}\left(x^{0}, y^{0} ; \mathbf{p}\right)=0,
$$

and similarly for the corresponding components of $F, A$ and $C$. We emphasize, however, that in the presence of spontaneous chiral symmetry breaking there is no such simplification.

In conclusion, for the above symmetry properties we are left with only four independent propagators: the two spectral functions $\rho_{V}^{0}\left(x^{0}, y^{0} ; \mathbf{p}\right)$ and $\rho_{V}\left(x^{0}, y^{0} ; \mathbf{p}\right)$ and the two corresponding statistical functions $F_{V}^{0}\left(x^{0}, y^{0} ; \mathbf{p}\right)$ and $F_{V}\left(x^{0}, y^{0} ; \mathbf{p}\right)$. They are either purely real or imaginary and have definite symmetry properties under the exchange of their time arguments $x^{0} \leftrightarrow y^{0}$. These properties as well as the corresponding ones for the various components of the self-energy are summarized below:

$\rho_{V}^{0}, A_{V}^{0}$ : imaginary, symmetric;

$\rho_{V}, A_{V}:$ real, antisymmetric;

$F_{V}^{0}, C_{V}^{0}: \quad$ imaginary, antisymmetric;

$F_{V}, C_{V}$ : real, symmetric.

The exact evolution equations for the spectral functions read (cf. Eq. (142)): ${ }^{9}$

$$
\begin{aligned}
& i \frac{\partial}{\partial x^{0}} \rho_{V}^{0}\left(x^{0}, y^{0} ; p\right)=p \rho_{V}\left(x^{0}, y^{0} ; p\right) \\
& \quad+\int_{y^{0}}^{x^{0}} \mathrm{~d} z^{0}\left[A_{V}^{0}\left(x^{0}, z^{0} ; p\right) \rho_{V}^{0}\left(z^{0}, y^{0} ; p\right)-A_{V}\left(x^{0}, z^{0} ; p\right) \rho_{V}\left(z^{0}, y^{0} ; p\right)\right] \\
& i \frac{\partial}{\partial x^{0}} \rho_{V}\left(x^{0}, y^{0} ; p\right)=p \rho_{V}^{0}\left(x^{0}, y^{0} ; p\right) \\
& \quad+\int_{y^{0}}^{x^{0}} \mathrm{~d} z^{0}\left[A_{V}^{0}\left(x^{0}, z^{0} ; p\right) \rho_{V}\left(z^{0}, y^{0} ; p\right)-A_{V}\left(x^{0}, z^{0} ; p\right) \rho_{V}^{0}\left(z^{0}, y^{0} ; p\right)\right] .
\end{aligned}
$$

\footnotetext{
${ }^{9}$ We note that the following equations do not rely on the restrictions (162) imposed by $C P$-invariance: they have the very same form for the case that all two-point functions are complex.
} 
Similarly, for the statistical two-point functions we obtain (cf. Eq. (142)):

$$
\begin{aligned}
& i \frac{\partial}{\partial x^{0}} F_{V}^{0}\left(x^{0}, y^{0} ; p\right)=p F_{V}\left(x^{0}, y^{0} ; p\right) \\
& +\int_{0}^{x^{0}} \mathrm{~d} z^{0}\left[A_{V}^{0}\left(x^{0}, z^{0} ; p\right) F_{V}^{0}\left(z^{0}, y^{0} ; p\right)-A_{V}\left(x^{0}, z^{0} ; p\right) F_{V}\left(z^{0}, y^{0} ; p\right)\right] \\
& \quad-\quad \int_{0}^{y^{0}} \mathrm{~d} z^{0}\left[C_{V}^{0}\left(x^{0}, z^{0} ; p\right) \rho_{V}^{0}\left(z^{0}, y^{0} ; p\right)-C_{V}\left(x^{0}, z^{0} ; p\right) \rho_{V}\left(z^{0}, y^{0} ; p\right)\right] \\
& i \frac{\partial}{\partial x^{0}} F_{V}\left(x^{0}, y^{0} ; p\right)=p F_{V}^{0}\left(x^{0}, y^{0} ; p\right) \\
& \quad+\quad \int_{0}^{x^{0}} \mathrm{~d} z^{0}\left[A_{V}^{0}\left(x^{0}, z^{0} ; p\right) F_{V}\left(z^{0}, y^{0} ; p\right)-A_{V}\left(x^{0}, z^{0} ; p\right) F_{V}^{0}\left(z^{0}, y^{0} ; p\right)\right] \\
& \quad-\quad \int_{0}^{y^{0}} \mathrm{~d} z^{0}\left[C_{V}^{0}\left(x^{0}, z^{0} ; p\right) \rho_{V}\left(z^{0}, y^{0} ; p\right)-C_{V}\left(x^{0}, z^{0} ; p\right) \rho_{V}^{0}\left(z^{0}, y^{0} ; p\right)\right] .
\end{aligned}
$$

The above equations are employed in Sec. 4 to calculate the nonequilibrium fermion dynamics in a chiral quark-meson model.

The time evolution for the fermions is described by first-order (integro-)differential equations for $F$ and $\rho$ : Eqs. (164)-(167). As pointed out above, the initial fermion spectral function is completely determined by the equal-time anticommutation relation of fermionic field operators (cf. Eq. (124)). To uniquely specify the time evolution for $F$ we have to set the initial conditions. The most general (Gaussian) initial conditions for $F$ respecting spatial homogeneity, isotropy, parity, charge conjugation and chiral symmetry can be written as

$$
\begin{aligned}
& \left.F_{V}\left(t, t^{\prime}, p\right)\right|_{t=t^{\prime}=0}=\frac{1}{2}-n_{0}^{f}(p), \\
& \left.F_{V}^{0}\left(t, t^{\prime}, p\right)\right|_{t=t^{\prime}=0}=0 .
\end{aligned}
$$

Here $n_{0}^{f}(p)$ denotes the initial particle number distribution, whose values can range between 0 and 1 .

\subsection{Nonequilibrium dynamics from the 2PI loop expansion}

In Sec. 3.4.3 we have derived coupled evolution equations (138) for the statistical propagator $F$ and the spectral function $\rho$. A systematic approximation to the exact equations can be obtained from the loop or coupling expansion of the 2PI effective action, as discussed in Sec. 2.1. This determines all the required self-energies using (25) and the decomposition identities (116) and (120) for scalars, and (121) for fermions. (Approximations for gauge field theories will be discussed in Sec. 6 in the context of $n \mathrm{PI}$ effective actions with $n>2$.) We emphasize that all classifications of contributions are done for the effective action. Once an approximation order is specified on the level of the effective action, there are no further classifications on the level of the evolution 
equations. This is a crucial aspect, which ensures the "conserving" properties of 2PI expansions (cf. the discussion in Sec. 1.1). The purpose of this section is to present the relevant formulae, whose physics will be explained in detail in the later sections.

We consider first the case of the scalar $O(N)$ symmetric field theory with classical action (13) and a vanishing field expectation value. The case $\phi \neq 0$ is treated in Sec. 3.6.1 below. From the 2PI effective action to three-loop order (36) one finds with $F_{a b}(x, y)=$ $F(x, y) \delta_{a b}$ and $\rho_{a b}(x, y)=\rho(x, y) \delta_{a b}$ the two-loop self-energies:

$$
\begin{aligned}
\Sigma^{(0)}(x) & =\lambda \frac{N+2}{6 N} F(x, x), \\
\Sigma_{\rho}(x, y) & =-\lambda^{2} \frac{N+2}{6 N^{2}} \rho(x, y)\left[F^{2}(x, y)-\frac{1}{12} \rho^{2}(x, y)\right], \\
\Sigma_{F}(x, y) & =-\lambda^{2} \frac{N+2}{18 N^{2}} F(x, y)\left[F^{2}(x, y)-\frac{3}{4} \rho^{2}(x, y)\right],
\end{aligned}
$$

which enter (119) and (138). The two-loop contribution to the effective action adds only to $\Sigma^{(0)}$ and corresponds to a space-time dependent mass shift in the evolution equations. As is discussed in detail below in Sec. 4.1, the time evolution obtained from the two-loop 2PI effective action (Hartree, or similarly leading-order large-N approximations) suffers from the presence of an infinite number of spurious conserved quantities, which are not present in the fully interacting theory. As an important consequence no thermalization can be observed to that order. A crucial ingredient for the description of nonequilibrium evolution comes from the three-loop contribution to the $2 \mathrm{PI}$ effective action as described by (171) and (172).

For the chiral Yukawa model for $N_{f}=2$ fermion flavors and $N_{f}^{2}$ scalar fields with classical action (68) as described in Sec. 2.3.1, one obtains from the two-loop 2PI effective action the fermion self-energies entering Eqs. (164)-(167):

$$
\begin{aligned}
A_{V}^{\mu}\left(x^{0}, y^{0} ; \mathbf{p}\right)= & -h^{2} \int \frac{\mathrm{d}^{3} q}{(2 \pi)^{3}}\left[F_{V}^{\mu}\left(x^{0}, y^{0} ; \mathbf{q}\right) \rho\left(x^{0}, y^{0} ; \mathbf{p}-\mathbf{q}\right)\right. \\
& \left.+\rho_{V}^{\mu}\left(x^{0}, y^{0} ; \mathbf{q}\right) F\left(x^{0}, y^{0} ; \mathbf{p}-\mathbf{q}\right)\right], \\
C_{V}^{\mu}\left(x^{0}, y^{0} ; \mathbf{p}\right)= & -h^{2} \int \frac{\mathrm{d}^{3} q}{(2 \pi)^{3}}\left[F_{V}^{\mu}\left(x^{0}, y^{0} ; \mathbf{q}\right) F\left(x^{0}, y^{0} ; \mathbf{p}-\mathbf{q}\right)\right. \\
& \left.-\frac{1}{4} \rho_{V}^{\mu}\left(x^{0}, y^{0} ; \mathbf{q}\right) \rho\left(x^{0}, y^{0} ; \mathbf{p}-\mathbf{q}\right)\right] .
\end{aligned}
$$

Here $\rho_{V}^{\mu}\left(x^{0}, y^{0} ; \mathbf{q}\right)$ and $F_{V}^{\mu}\left(x^{0}, y^{0} ; \mathbf{q}\right)$ are the vector components of the fermion two-point functions as introduced in Sec. 3.4.5. The dynamics of the scalar two-point functions $\rho\left(x^{0}, y^{0} ; \mathbf{p}\right)$ and $F\left(x^{0}, y^{0} ; \mathbf{p}\right)$ is described by the evolution equations (138) with the scalar self-energies to this loop order:

$$
\Sigma^{(0)}(x)=\lambda \frac{N_{f}^{2}+2}{6 N_{f}^{2}} \int \frac{\mathrm{d}^{3} q}{(2 \pi)^{3}} F\left(x^{0}, x^{0} ; \mathbf{q}\right),
$$




$$
\begin{aligned}
\Sigma_{\rho}\left(x^{0}, y^{0} ; \mathbf{p}\right)= & -\frac{8 h^{2}}{N_{f}} \int \frac{\mathrm{d}^{3} q}{(2 \pi)^{3}} \rho_{V}^{\mu}\left(x^{0}, y^{0} ; \mathbf{q}\right) F_{V, \mu}\left(x^{0}, y^{0} ; \mathbf{p}-\mathbf{q}\right), \\
\Sigma_{F}\left(x^{0}, y^{0} ; \mathbf{p}\right)= & -\frac{4 h^{2}}{N_{f}} \int \frac{\mathrm{d}^{3} q}{(2 \pi)^{3}}\left[F_{V}^{\mu}\left(x^{0}, y^{0} ; \mathbf{q}\right) F_{V, \mu}\left(x^{0}, y^{0} ; \mathbf{p}-\mathbf{q}\right)\right. \\
& \left.-\frac{1}{4} \rho_{V}^{\mu}\left(x^{0}, y^{0} ; \mathbf{q}\right) \rho_{V, \mu}\left(x^{0}, y^{0} ; \mathbf{p}-\mathbf{q}\right)\right] .
\end{aligned}
$$

\subsection{Nonequilibrium dynamics from the $2 \mathrm{PI} 1 / N$ expansion}

We consider first the case of the scalar $O(N)$ symmetric field theory with classical action (13) and a vanishing field expectation value such that $F_{a b}(x, y)=F(x, y) \delta_{a b}$ and $\rho_{a b}(x, y)=\rho(x, y) \delta_{a b}$. The case $\phi \neq 0$ is treated in Sec. 3.6.1. In the $1 / N$-expansion of the 2PI effective action to next-to-leading order, as discussed in Sec. 2.4, the effective mass term $M^{2}(x ; G)$ appearing in the evolution equations (138) is given by

$$
M^{2}(x ; F)=m^{2}+\lambda \frac{N+2}{6 N} F(x, x) .
$$

One observes that this local self-energy part receives LO and NLO contributions. In contrast, the non-local part of the self-energy (118) is nonvanishing only at NLO: $\bar{\Sigma}(x, y ; G)=-\lambda /(3 N) G(x, y) I(x, y)$ and using the decomposition identities (116) and (120) one finds

$$
\begin{aligned}
& \Sigma_{F}(x, y)=-\frac{\lambda}{3 N}\left(F(x, y) I_{F}(x, y)-\frac{1}{4} \rho(x, y) I_{\rho}(x, y)\right) \\
& \Sigma_{\rho}(x, y)=-\frac{\lambda}{3 N}\left(F(x, y) I_{\rho}(x, y)+\rho(x, y) I_{F}(x, y)\right) .
\end{aligned}
$$

Here the summation function (84) reads in terms of its statistical and spectral components: ${ }^{10}$

$$
\begin{aligned}
I_{F}(x, y) & =\frac{\lambda}{6}\left(F^{2}(x, y)-\frac{1}{4} \rho^{2}(x, y)\right) \\
-\frac{\lambda}{6}\left\{\int_{0}^{x^{0}} \mathrm{~d} z I_{\rho}(x, z)\left(F^{2}(z, y)-\frac{1}{4} \rho^{2}(z, y)\right)-2 \int_{0}^{y^{0}} \mathrm{~d} z I_{F}(x, z) F(z, y) \rho(z, y)\right\}, & \\
I_{\rho}(x, y) & =\frac{\lambda}{3} F(x, y) \rho(x, y)-\frac{\lambda}{3} \int_{y^{0}}^{x^{0}} \mathrm{~d} z I_{\rho}(x, z) F(z, y) \rho(z, y),
\end{aligned}
$$

\footnotetext{
10 This follows from using the decomposition identity for the propagator (116) and $I(x, y)=I_{F}(x, y)-$ $\frac{i}{2} I_{\rho}(x, y) \operatorname{sign}_{\mathscr{C}}\left(x^{0}-y^{0}\right)$. Cf. also the detailed discussion in Sec. 3.4.3.
} 
using the abbreviated notation $\int_{t_{1}}^{t_{2}} \mathrm{~d} z \equiv \int_{t_{1}}^{t_{2}} \mathrm{~d} z^{0} \int_{-\infty}^{\infty} \mathrm{d}^{d} z$. Note that $F(x, y), \rho(x, y)$, $\Sigma_{F}(x, y), \Sigma_{\rho}(x, y), I_{F}(x, y)$ and $I_{\rho}(x, y)$ are real functions.

For the Yukawa model of Sec. 2.3.1 the results from the $1 / N_{f}$ expansion of the 2PI effective action to NLO can be directly inferred from (173)-(177). Recall that all classifications are done on the level of the effective action, as explained for fermions at the end of Sec. 2.4.1. The only difference between the NLO evolution equations and the result (173)-(177) from the two-loop effective action is that for the former the NNLO part $\sim N_{f}^{-2}$ in (175) is dropped.

\subsubsection{Nonvanishing field expectation value}

We consider the scalar $N$-component field theory with classical action (13). In the presence of a nonzero field expectation value $\phi_{a}$ the most general propagator can no longer be evaluated for the diagonal configuration (35). Restoring all field indices the evolution equations (138) read

$$
\begin{aligned}
{\left[\square_{x} \delta_{a c}+M_{a c}^{2}(x)\right] \rho_{c b}(x, y)=} & -\int_{y^{0}}^{x^{0}} \mathrm{~d} z \Sigma_{a c}^{\rho}(x, z) \rho_{c b}(z, y), \\
{\left[\square_{x} \delta_{a c}+M_{a c}^{2}(x)\right] F_{c b}(x, y)=} & -\int_{0}^{x^{0}} \mathrm{~d} z \Sigma_{a c}^{\rho}(x, z) F_{c b}(z, y) \\
& +\int_{0}^{y^{0}} \mathrm{~d} z \Sigma_{a c}^{F}(x, z) \rho_{c b}(z, y) .
\end{aligned}
$$

The statistical propagator and spectral function components have the properties $F_{a b}^{*}(x, y)=F_{a b}(x, y)=F_{b a}(y, x)$ and $\rho_{a b}^{*}(x, y)=\rho_{a b}(x, y)=-\rho_{b a}(y, x)$. At NLO in the 2PI $1 / N$ expansion $M_{a b}^{2}(x) \equiv M_{a b}^{2}(x ; \phi, F)$ is given by (146) and the self-energies $\Sigma_{a b}^{F}(x, y) \equiv \Sigma_{a b}^{F}(x, y ; \phi, \rho, F)$ and $\Sigma_{a b}^{\rho}(x, y) \equiv \Sigma_{a b}^{\rho}(x, y ; \phi, \rho, F)$ are obtained from (83) as

$$
\begin{gathered}
\Sigma_{a b}^{F}(x, y)=-\frac{\lambda}{3 N}\left\{I_{F}(x, y)\left[\phi_{a}(x) \phi_{b}(y)+F_{a b}(x, y)\right]-\frac{1}{4} I_{\rho}(x, y) \rho_{a b}(x, y)\right. \\
\left.+P_{F}(x, y) F_{a b}(x, y)-\frac{1}{4} P_{\rho}(x, y) \rho_{a b}(x, y)\right\}, \\
\Sigma_{a b}^{\rho}(x, y)=-\frac{\lambda}{3 N}\left\{\begin{array}{l}
I_{\rho}(x, y)\left[\phi_{a}(x) \phi_{b}(y)+F_{a b}(x, y)\right]+I_{F}(x, y) \rho_{a b}(x, y) \\
\left.+P_{\rho}(x, y) F_{a b}(x, y)+P_{F}(x, y) \rho_{a b}(x, y)\right\} .
\end{array}\right.
\end{gathered}
$$

The functions $I_{F}(x, y) \equiv I_{F}(x, y ; \rho, F)$ and $I_{\rho}(x, y) \equiv I_{\rho}(x, y ; \rho, F)$ satisfy the same equations as for the case of a vanishing macroscopic field given by (181) and (182). The respective $\phi$-dependent summation functions $P_{F}(x, y) \equiv P_{F}(x, y ; \phi, \rho, F)$ and $P_{\rho}(x, y) \equiv$ $P_{\rho}(x, y ; \phi, \rho, F)$ are given by

$$
P_{F}(x, y)=-\frac{\lambda}{3 N}\left\{H_{F}(x, y)-\int_{0}^{x^{0}} \mathrm{~d} z\left[H_{\rho}(x, z) I_{F}(z, y)+I_{\rho}(x, z) H_{F}(z, y)\right]\right.
$$




$$
\begin{aligned}
& +\int_{0}^{y^{0}} \mathrm{~d} z\left[H_{F}(x, z) I_{\rho}(z, y)+I_{F}(x, z) H_{\rho}(z, y)\right] \\
& -\int_{0}^{x^{0}} \mathrm{~d} z \int_{0}^{y^{0}} \mathrm{~d} v I_{\rho}(x, z) H_{F}(z, v) I_{\rho}(v, y) \\
& +\int_{0}^{x^{0}} \mathrm{~d} z \int_{0}^{z^{0}} \mathrm{~d} v I_{\rho}(x, z) H_{\rho}(z, v) I_{F}(v, y) \\
& \left.+\int_{0}^{y^{0}} \mathrm{~d} z \int_{z^{0}}^{y^{0}} \mathrm{~d} v I_{F}(x, z) H_{\rho}(z, v) I_{\rho}(v, y)\right\}, \\
P_{\rho}(x, y)= & -\frac{\lambda}{3 N}\left\{H_{\rho}(x, y)-\int_{y^{0}}^{x^{0}} \mathrm{~d} z\left[H_{\rho}(x, z) I_{\rho}(z, y)+I_{\rho}(x, z) H_{\rho}(z, y)\right]\right. \\
& \left.+\int_{y^{0}}^{x^{0}} \mathrm{~d} z \int_{y^{0}}^{z^{0}} \mathrm{~d} v I_{\rho}(x, z) H_{\rho}(z, v) I_{\rho}(v, y)\right\},
\end{aligned}
$$

with

$$
H_{F}(x, y) \equiv-\phi_{a}(x) F_{a b}(x, y) \phi_{b}(y), \quad H_{\rho}(x, y) \equiv-\phi_{a}(x) \rho_{a b}(x, y) \phi_{b}(y) .
$$

The time evolution equation for the field (147) for the 2PI effective action to NLO (83) is given by

$$
\begin{gathered}
\left(\left[\square_{x}+\frac{\lambda}{6 N} \phi^{2}(x)\right] \delta_{a b}+M_{a b}^{2}(x ; \phi=0, F)\right) \phi_{b}(x) \\
=\frac{\lambda}{3 N} \int_{0}^{x^{0}} \mathrm{~d} y\left[I_{\rho}(x, y) F_{a b}(x, y)+I_{F}(x, y) \rho_{a b}(x, y)\right] \phi_{b}(y) \\
=-\int_{0}^{x^{0}} \mathrm{~d} y \Sigma_{a b}^{\rho}(x, y ; \phi=0, F, \rho) \phi_{b}(y) .
\end{gathered}
$$

The second equality follows from (185).

\subsection{Numerical implementation}

Beyond the leading-order approximation, the time evolution equations (138) and (147) are nonlinear integro-differential equations. The approximate self-energies obtained from a loop expansion or $1 / N$ expansion of the 2PI effective action are described in Secs. 3.5 and 3.6. Though these equations are in general too complicated to be solved analytically ${ }^{11}$ without additional approximations, they can be very efficiently implemented and solved on a computer. Here it is important to note that all equations are

${ }^{11} \mathrm{Cf}$. Sec. 4.3 for an approximate analytical solution in the context of parametric resonance. 
explicit in time, i.e. all quantities at some later time $t_{f}$ can be obtained by integration over the explicitly known functions for times $t<t_{f}$ for given initial conditions. This is a direct consequence of causality. In this respect, solving the nonequilibrium evolution equations can be technically simpler than solving the corresponding theory in thermal equilibrium. The reason is that for the study of thermal equilibrium the equation of the form (111) is typically employed, which has to be solved self-consistently since on its 1.h.s. and r.h.s. the same variables appear. It involves the propagator for the full range of its arguments. In contrast, the time-evolution equations (138) for $\left.\rho(x, y)\right|_{x^{0}=t_{1}, y^{0}=t_{2}}$ and $\left.F(x, y)\right|_{x^{0}=t_{1}, y^{0}=t_{2}}$ do not depend on the r.h.s. on $\rho(x, y)$ and $F(x, y)$ for times $x^{0} \geq t_{1}$ and $y^{0} \geq t_{2}$. To see this note that the integrands vanish identically for the upper time-limits of the memory integrals because of the anti-symmetry of the spectral components, with $\left.\rho(x, y)\right|_{x^{0}=y^{0}} \equiv 0$ and $\left.\Sigma_{\rho}(x, y)\right|_{x^{0}=y^{0}} \equiv 0$. As a consequence, only explicitly known quantities at earlier times determine the time evolution of the unknowns at later times. The numerical implementation therefore only involves sums over known functions.

To be more explicit we consider first a scalar field theory. For spatially homogeneous fields it is sufficient to implement the equations for the Fourier components $F\left(t, t^{\prime} ; \mathbf{p}\right)$ and $\rho\left(t, t^{\prime} ; \mathbf{p}\right)$ and we consider $\phi=0$. The numerical implementation with $\phi \neq 0$ follows along the very same lines for the equation (147). Spatially inhomogeneous fields pose no complication in principle but are computationally more expensive. As an example we consider a time discretization $t=n a_{t}, t^{\prime}=m a_{t}$ with stepsize $a_{t}$ such that $F\left(t, t^{\prime}\right) \mapsto$ $F(n, m)$, and

$$
\begin{aligned}
\partial_{t}^{2} F\left(t, t^{\prime}\right) & \mapsto \frac{1}{a_{t}^{2}}(F(n+1, m)+F(n-1, m)-2 F(n, m)), \\
\int_{0}^{t} d t F\left(t, t^{\prime}\right) & \mapsto a_{t}\left(F(0, m) / 2+\sum_{l=1}^{n-1} F(l, m)+F(n, m) / 2\right),
\end{aligned}
$$

where we have suppressed the momentum labels in the notation. The second derivative is replaced by a finite-difference expression, which is symmetric in $a_{t} \leftrightarrow-a_{t}$. It is obtained from employing subsequently the lattice "forward derivative" $[F(n+1, m)-F(n, m)] / a_{t}$ and "backward derivative" $[F(n, m)-F(n-1, m)] / a_{t}$. The integral is approximated using the trapezoidal rule with the average function value $[F(n, m)+F(n+1, m)] / 2$ in an interval of length $a_{t}$. The above simple discretization leads already to stable numerics for small enough stepsize $a_{t}$, but the convergence properties may be easily improved with more sophisticated standard estimators if required.

As for the continuum the propagators obey the symmetry properties $F(n, m)=$ $F(m, n)$ and $\rho(n, m)=-\rho(m, n)$. Consequently, only "half" of the $(n, m)$-matrices have to be computed and $\rho(n, n) \equiv 0$. Similarly, since the self-energy $\Sigma_{\rho}$ and the summation function $I_{\rho}$ appearing in the $1 / N$ expansion to NLO (cf. Eq. (182)) are antisymmetric in time one can exploit that $\Sigma_{\rho}(n, n)$ and $I_{\rho}(n, n)$ vanish identically. For the case of the NLO time evolution the equations (138) with (179)-(182) are used to advance the matrices $F(n, m)$ and $\rho(n, m)$ stepwise in the " $n$-direction" for each given $m$. As initial conditions one has to specify $F(0,0 ; \mathbf{p}), F(1,0 ; \mathbf{p})$ and $F(1,1 ; \mathbf{p})$, while $\rho(0,0 ; \mathbf{p})$, $\rho(1,0 ; \mathbf{p})$ and $\rho(1,1 ; \mathbf{p})$ are fixed by the equal-time commutation relations (117). The 
time discretized versions of (138) read:

$$
\begin{aligned}
F(n+1, & m ; \mathbf{p})=2 F(n, m ; \mathbf{p})-F(n-1, m ; \mathbf{p}) \\
-a_{t}^{2} & \left\{\mathbf{p}^{2}+m^{2}+\lambda \frac{N+2}{6 N} \int_{\mathbf{k}} F(n, n ; \mathbf{k})\right\} F(n, m ; \mathbf{p}) \\
-a_{t}^{3}\left\{\Sigma_{\rho}(n, 0 ; \mathbf{p}) F(0, m ; \mathbf{p}) / 2-\Sigma_{F}(n, 0 ; \mathbf{p}) \rho(0, m ; \mathbf{p}) / 2\right. & \\
& +\sum_{l=1}^{m-1}\left(\Sigma_{\rho}(n, l ; \mathbf{p}) F(l, m ; \mathbf{p})-\Sigma_{F}(n, l ; \mathbf{p}) \rho(l, m ; \mathbf{p})\right) \\
& \left.+\sum_{l=m}^{n-1} \Sigma_{\rho}(n, l ; \mathbf{p}) F(l, m ; \mathbf{p})\right\},
\end{aligned}
$$

$(n \geq m)^{12}$ and similarly for $\rho$. These equations are explicit in time: Starting with $n=1$, for the time step $n+1$ one computes successively all entries with $m=0, \ldots, n+1$ from known functions at earlier times. At first sight this property is less obvious for the nonderivative expressions (181) for $I_{F}$ and (182) for $I_{\rho}$ whose form is reminiscent of a gap equation. However, the discretized equation for $I_{\rho}$,

$$
\begin{aligned}
& I_{\rho}(n, m ; \mathbf{q})=\frac{\lambda}{3} \int_{\mathbf{k}}\{F(n, m ; \mathbf{q}-\mathbf{k}) \rho(n, m ; \mathbf{k}) \\
& \left.\quad-a_{t} \sum_{l=m+1}^{n-1} I_{\rho}(n, l ; \mathbf{q}) F(l, m ; \mathbf{q}-\mathbf{k}) \rho(l, m ; \mathbf{k})\right\},
\end{aligned}
$$

shows that all expressions for $I_{\rho}(n, m)$ are explicit as well: Starting with $m=n$ where $I_{\rho}$ vanishes one should lower $m=n, \ldots, 0$ successively. For $m=n-1$ one obtains an explicit expression in terms of $F(n, m)$ and $\rho(n, m)$ known from the previous time step in $n$. For $m=n-2$ the r.h.s. then depends on the known function $I_{\rho}(n, n-1)$ and so on. Similarly, for given $I_{\rho}(n, m)$ it is easy to convince oneself that the discretized Eq. (182) specifies $I_{F}(n, m)$ completely in terms of $I_{F}(n, 0), \ldots, I_{F}(n, m-1)$, which constitutes an explicit set of equations by increasing $m$ successively from zero to $n$.

It is crucial for an efficient numerical implementation that each step forward in time does not involve the solution of a self consistent or gap equation. This is manifest in the above discretization. The main numerical limitation of the approach is set by the time integrals ("memory integrals") which grow with time and therefore slow down the numerical evaluation. Typically, the influence of early times on the late time behavior is suppressed and can be neglected numerically in a controlled way. In this case, it is often sufficient to only take into account the contributions from the memory integrals

\footnotetext{
${ }^{12}$ For the discretization of the time integrals it is useful to distinguish the cases $n \geq m$ and $n \leq m$. We compute the entries $F(n+1, m)$ from the discretized equations for $n \geq m$ except for $n+1=m$ where we have to use the equations for $n \leq m$.
} 
for times much larger then the characteristic inverse damping rate (cf. Sec. 4.1.4 below). An error estimate then involves a series of runs with increasing memory time.

For scalars one can use a standard lattice discretization for a spatial volume with periodic boundary conditions. For a spatial volume $V=\left(N_{s} a\right)^{d}$ with lattice spacing $a$ one finds for the momenta:

$$
\mathbf{p}^{2} \mapsto \sum_{i=1}^{d} \frac{4}{a^{2}} \sin ^{2}\left(\frac{a p_{i}}{2}\right), \quad p_{i}=\frac{2 \pi n_{i}}{N_{s} a},
$$

where $n_{i}=0, \ldots, N_{s}-1$. This can be easily understood from acting with the corresponding finite-difference expression (190) for space-components: $\partial_{x}^{2} e^{-i p x} \mapsto e^{-i p x}\left[e^{i p a}+\right.$ $\left.e^{-i p a}-2\right] / a^{2}=-e^{-i p x} 4 \sin ^{2}(p a / 2) / a^{2}$. On the lattice there is only a subgroup of the rotation symmetry generated by the permutations of $p_{x}, p_{y}, p_{z}$ and the reflections $p_{x} \leftrightarrow-p_{x}$ etc. for $d=3$. Exploiting these lattice symmetries reduces the number of independent lattice sites to $\left(N_{s}+1\right)\left(N_{s}+3\right)\left(N_{s}+5\right) / 48$. We emphasize that the self-energies are calculated in coordinate space, where they are given by products of coordinate-space correlation functions, and then transformed back to momentum space. The coordinate-space correlation functions are available by fast Fourier transformation routines.

The lattice introduces a momentum cutoff $\pi / a$, however, the renormalized quantities are insensitive to cutoff variations for sufficiently large $\pi / a$. We emphasize here again that it is often convenient to carry out the numerical calculations using unrenormalized equations, or equations where only the dominant (quadratically) divergent contributions in the presence of scalars are subtracted. Cf. the discussion at the end of Sec. 2.2. In order to study the infinite volume limit one has to remove finite size effects. Here this is done by increasing the volume until convergence of the results is observed. ${ }^{13}$ We finally note that the nonequilibrium equations are very suitable for parallel computing on PC clusters using the MPI standard.

For fermionic field theories the numerical implementation is more involved than for purely scalar theories. Consider the evolution equations for the Yukawa model (164)(167), together with the self-energies (173)-(174). The structure of the fermionic equations is reminiscent of the form of classical canonical equations. In this analogy, $F_{V}\left(t, t^{\prime}\right)$ plays the role of the canonical coordinate and $F_{V}^{0}\left(t, t^{\prime}\right)$ is analogous to the canonical momentum. This suggests to discretize $F_{V}\left(t, t^{\prime}\right)$ and $\rho_{V}\left(t, t^{\prime}\right)$ at $t-t^{\prime}=2 n a_{t}$ (even) and

\footnotetext{
13 For time evolution problems the volume which is necessary to reach the infinite volume limit to a given accuracy can depend on the time scale. This is, in particular, due to the fact that finite systems can show characteristic recurrence times after which an initial effective damping of oscillations can be reversed. The observed damping can be viewed as the result of a superposition of oscillatory functions with differing phases or frequencies. The recurrence time is given by the time after which the phase information contained in the initial oscillations is recovered. Then the damping starts again until twice the recurrence time is reached and so on. In the LO approximation one can explicitly verify that the observed recurrence times e.g. for the correlation $F(x, x)$ scales with the volume or the number of lattice sites to "infinity". We emphasize that the phenomenon of complete recurrences, repeating the full initial oscillation pattern after some characteristic time, is not observed once scattering is taken into account. Periodic recurrences can occur with smaller amplitudes as time proceeds and are effectively suppressed in the large-time limit.
} 
$F_{V}^{0}\left(t, t^{\prime}\right)$ and $\rho_{V}^{0}\left(t, t^{\prime}\right)$ at $t-t^{\prime}=(2 n+1) a_{t}$ (odd) time-like lattice sites with spacing $a_{t}$. This is a generalization of the "leap-frog" prescription for temporally inhomogeneous two-point functions. This implies in particular that the discretization in the time direction is coarser for the fermionic two-point functions than for the bosonic ones. This "leap-frog" prescription may be easily extended to the memory integrals on the r.h.s. of Eqs. (164)-(167) as well.

We emphasize that the discretization does not suffer from the problem of so-called fermion doublers. The spatial doublers do not appear since (164)-(167) are effectively

second order in $\vec{x}$-space. Writing the equations for $\vec{F}_{V}\left(t, t^{\prime}, \vec{x}\right)$ and $\vec{\rho}_{V}\left(t, t^{\prime}, \vec{x}\right)$ starting from (164)-(167) one realizes that instead of first order spatial derivatives there is a Laplacian appearing. Hence we have the same Brillouin zone for the fermions and scalars. Moreover, time-like doublers are easily avoided by using a sufficiently small stepsize in time $a_{t} / a_{s}$.

The fact that Eqs. (164)-(167) with (173)-(174) and the respective scalar ones contain memory integrals makes numerical implementations expensive. Within a given numerical precision it is typically not necessary to keep all the past of the two-point functions in the memory. A single PIII desktop workstation with $2 \mathrm{~GB}$ memory allows us to use a memory array with 470 time-steps (with 2 temporal dimensions: $t$ and $t^{\prime}$ ). For instance, we have checked for the presented runs in Sec. 4.2 that a $30 \%$ change in the memory interval length did not alter the results. For a typical run 1-2 CPU-days were necessary. Much shorter times can be achieved with parallel computing on PC clusters using the MPI standard. The shown plots in that section are calculated on a $470 \times 470 \times 32^{3}$ lattice. (The dimensions refer to the $t$ and $t^{\prime}$ memory arrays and the momentum-space discretization, respectively.) By exploiting the spatial symmetries described in Sec. 3.4.5 the memory need could be reduced by a factor of 30 . We have checked that the infrared cutoff is well below any other mass scales and that the UV cutoff is greater than the mass scales at least by a factor of three. In the evolution equations we analytically subtract only the respective quadratically divergent terms. To extract physical quantities we follow the time evolution of the system for a given lattice cutoff and measure e.g. the renormalized scalar mass from the oscillation frequency of the correlator zero-modes to set the scale.

\section{NONEQUILIBRIUM PHENOMENA}

Our aim in this section is to study quantum field theories which capture important aspects of nonequilibrium physics and which are simple enough that one can perform a precise quantitative treatment. We employ first the two-particle irreducible $1 / N$ expansion for the scalar $N$-component field theory (13). Considering subsequent orders in the expansion corresponds to include more and more aspects of the dynamics. This allows one to study the influence of characteristic ingredients such as scattering, off-shell and memory effects on the time evolution. We note that time-reflection invariance for the 
equations and conservation of energy is preserved at each order in the $1 / N$-expansion. ${ }^{14}$ It should be stressed that during the nonequilibrium time evolution there is no loss of information in any strict sense. Here we consider closed systems without coupling to an external heat bath or external fields. There is no course graining or averaging involved in the dynamics. The important process of thermalization is a nontrivial question in a calculation from first principles. Thermal equilibrium itself is time-translation invariant and cannot be reached from a nonequilibrium evolution on a fundamental level. It is striking that we will observe below that scattering drives the evolution very closely towards thermal equilibrium results without ever deviating from them for accessible times.

Here we study the time evolution for various initial condition scenarios away from equilibrium. One corresponds to a "quench" often employed to mimic the situation of a rapidly expanding hot initial state which cools on time scales much smaller than the relaxation time of the fields. Initially at high temperature we consider the relaxation processes following an instant "cooling" described by a sudden drop in the effective mass. Another scenario is characterized by initially densely populated modes in a narrow momentum range around $\pm \mathbf{p}_{\text {ts }}$. This so-called "tsunami" initial condition is reminiscent of colliding wave packets moving with opposite and equal momentum. A similar nonthermal and radially symmetric distribution of highly populated modes may also be encountered in the so-called "color glass" state at saturated gluon density. Of course, a sudden change in the two-point function of a previously equilibrated system or a peaked initial particle number distribution are general enough to exhibit characteristic properties of nonequilibrium dynamics for a large variety of physical situations. We will first consider results for the $1+1$ dimensional quantum field theory, since technical aspects such as the infinite volume limit, renormalization and large times can be all implemented with great rigour. The study also has the advantage that the physics of offshell effects can be very clearly discussed since they play a more important role for one spatial dimension as compared to three dimensions. The conclusions we will draw are not specific to scalar theories in low dimensions, and we will compare below with the corresponding results in $3+1$ dimensions for scalars interacting with fermions.

For the 2PI 1/ $N$ expansion we will first consider the time evolution at leading order (LO) and compare it with the next-to-leading order dynamics (NLO). The "mean-field type" LO approximation has a long history in the literature. However, a drastic consequence of this approximation is the appearance of an infinite number of conserved quantities, which are not present in the interacting finite- $N$ theory. These additional constants of motion can have a substantial impact on the time evolution, since they strongly constrain the allowed dynamics. As an important consequence of the LO approximation, the late-time behavior depends explicitly on the details of the initial conditions and the approach to thermal equilibrium cannot be observed in this case as is shown below. The extensive use of this approximation was based on the hope that deviations from the LO behavior are not too sizeable for not too late times. By taking into account the NLO contributions it turns out, however, that for many important questions there are substantial corrections long before thermalization sets in. In contrast, we will find that various approximations of the 2PI effective action which go beyond "mean-field type" (LO,

\footnotetext{
14 These properties are also taken care of by the employed numerical techniques.
} 
Hartree or any Gaussian) dynamics show thermalization and give comparable answers. The 2PI approximations beyond mean-field share the property that the spurious constants of motion are absent, which emphasizes the important role of conservation laws for the nonequilibrium dynamics: fake conserved quantities keep the information about the initial conditions for all times and can spoil any effective loss of details about the early-time behavior.

\subsection{Scattering, off-shell and memory effects}

\subsubsection{LO fixed points}

For simplicity we consider spatially homogeneous field expectation values $\phi_{a}(t)=$ $\left\langle\Phi_{a}(t, \mathbf{x})\right\rangle$, such that we can use the Fourier modes $F_{a b}\left(t, t^{\prime} ; \mathbf{p}\right)$ and $\rho_{a b}\left(t, t^{\prime} ; \mathbf{p}\right) .{ }^{15}$ The solution of (138) in the limit of a free field theory $(\lambda \equiv 0)$ describes modes which oscillate with frequency $\sqrt{\mathbf{p}^{2}+m^{2}} / 2 \pi$ for unequal-time functions $F(t, 0 ; \mathbf{p})$ and $\rho(t, 0 ; \mathbf{p})$, unless they are not identically zero. Equal-time correlation modes $F(t, t ; \mathbf{p})$ oscillate either with twice that frequency or they are constant in time $(\rho(t, t ; \mathbf{p}) \equiv 0$ according to Eq. (117)). The latter corresponds to solutions which are translationally invariant in time, i.e. $F\left(t, t^{\prime} ; \mathbf{p}\right)=F\left(t-t^{\prime} ; \mathbf{p}\right)$ and $\rho\left(t, t^{\prime} ; \mathbf{p}\right)=\rho\left(t-t^{\prime} ; \mathbf{p}\right)$.

The LO contribution to the 2 PI effective action (78) adds a time-dependent mass shift to the free field evolution equation. The resulting effective mass term, given by (146) for $N \rightarrow \infty$, is the same for all Fourier modes and consequently each mode propagates "collisionlessly". There are no further corrections since according to (79) and (83) the self-energies $\Sigma_{F}$ and $\Sigma_{\rho}$ are $\mathscr{O}(1 / N)$ and vanish in this limit! The evolution equations (138) for this approximation read:

$$
\begin{aligned}
{\left[\partial_{t}^{2}+\mathbf{p}^{2}+M^{2}(t ; \phi, F)\right] F_{a b}\left(t, t^{\prime} ; \mathbf{p}\right) } & =0, \\
{\left[\partial_{t}^{2}+\mathbf{p}^{2}+M^{2}(t ; \phi, F)\right] \rho_{a b}\left(t, t^{\prime} ; \mathbf{p}\right) } & =0, \\
{\left[\partial_{t}^{2}+\frac{\lambda}{6 N} \phi^{2}(t)+M^{2}(t ; \phi \equiv 0, F)\right] \phi_{b}(t) } & =0
\end{aligned}
$$

with

$$
M^{2}(t ; \phi, F) \equiv m^{2}+\frac{\lambda}{6 N}\left[\int_{\mathbf{p}} F_{c c}(t, t ; \mathbf{p})+\phi^{2}(t)\right],
$$

where $\int_{\mathbf{p}} \equiv \int \mathrm{d}^{d} p /(2 \pi)^{d}$. In this case one observes that the evolution of $F$ and $\phi$ is completely decoupled from $\rho$. Similar to the free field theory limit, to LO the spectral function does not influence the time evolution of the statistical propagator. The reason is that in this approximation the spectrum consists only of "quasiparticle" modes of energy $\omega_{\mathbf{p}}(t)=\sqrt{\mathbf{p}^{2}+M^{2}(t)}$ with an infinite life-time. The associated mode particle numbers

\footnotetext{
15 Note that a spatially homogeneous field expectation value contains all fluctuations arising from an inhomogeneous $\Phi(x)$ as well. From a practical point of view the extraction of physics related to inhomogeneous field fluctuations is, of course, more difficult since it is contained in higher correlation functions.
} 
are conserved for each momentum separately. In contrast, in the interacting quantum field theory particles decay, get created, annihilated and the notion of a conserved particle number is clearly absent for real scalar fields. Note that there are also no memory integrals appearing on the r.h.s. of (195), which incorporate in particular direct scattering processes.

As an example we consider a "tsunami" type initial condition in $1+1$ dimension. The initial statistical propagator is given by

$$
\begin{aligned}
& F(0,0 ; p)=\frac{n_{p}(0)+1 / 2}{\sqrt{p^{2}+M^{2}(0)}} \quad,\left.\quad \partial_{t} F(t, 0 ; p)\right|_{t=0}=0 \\
& \left.F(0,0 ; p) \partial_{t} \partial_{t^{\prime}} F\left(t, t^{\prime} ; p\right)\right|_{t=t^{\prime}=0}=\left[n_{p}(0)+1 / 2\right]^{2}, \\
& \phi(0)=\left.\partial_{t} \phi(t)\right|_{t=0}=0 .
\end{aligned}
$$

Here $F_{a b}\left(t, t^{\prime} ; \mathbf{p}\right) \equiv F\left(t, t^{\prime} ; \mathbf{p}\right) \delta_{a b}$, which is valid for all times with these initial conditions. The mass term $M^{2}(0)$ is given by the gap equation (196) in the presence of the initial non-thermal particle number distribution

$$
n_{p}(0)=\mathscr{A} e^{-\frac{1}{2 \sigma^{2}}\left(|p|-p_{\mathrm{ts}}\right)^{2}} .
$$

As the renormalization condition we choose the initial renormalized mass in vacuum, $\left.m_{R} \equiv M(0)\right|_{n(0)=0}=1$, as our dimensionful scale. There is no corresponding coupling renormalization in $1+1$ dimension. In these units the particle number is peaked around $|p|=p_{\mathrm{ts}}=5 m_{R}$ with a width determined by $\sigma=0.5 m_{R}$ and amplitude $\mathscr{A}=10$. We consider the effective coupling $\lambda /\left(6 m_{R}^{2}\right)=1$.

On the left of Fig. 3 we present the time evolution of the equal-time correlation modes $F(t, t ; p)$ for different momenta: zero momentum, a momentum close to the maximally populated momentum $p_{\mathrm{ts}}$ and about twice $p_{\mathrm{ts}}$. One observes that the equaltime correlations at LO are strictly constant in time. This behavior can be understood from the fact that for the employed "tsunami" initial condition the evolution starts at a time-translation invariant non-thermal solution of the LO equations. There is an infinite number of so-called fixed point solutions which are constant in time. If the real world would be well approximated by the LO dynamics then this would have dramatic consequences. The thermal equilibrium solution, which is obtained for an initial BoseEinstein distribution instead of (199), is not at all particular in this case! Therefore, everything depends on the chosen initial condition details. This is in sharp contrast to the well-founded expectation that the late-time behavior may be well described by thermal equilibrium physics. Scattering effects included in the NLO approximation indeed drive the evolution away from the LO non-thermal fixed points towards thermal equilibrium, which is discussed in detail in Sec. 4.1.2 below.

A remaining question is what happens at LO if the time evolution does not start from a LO fixed point, as was the case for the "tsunami" initial condition above. In the left graph of Fig. 4 we plot the evolution of $M^{2}(t)$ in the LO approximation as a function of time $t$, following a "quench" described by an instant drop in the effective mass term from $2 M^{2}(0)$ to $M^{2}(0)$. The initial particle number distribution is $n_{p}(0)=$ $1 /\left(\exp \left[\sqrt{p^{2}+2 M^{2}(0)} / T_{0}\right]-1\right)$ with $T_{0}=2 M(0)$ and $\phi(0)=\dot{\phi}(0)=0$. The sudden 

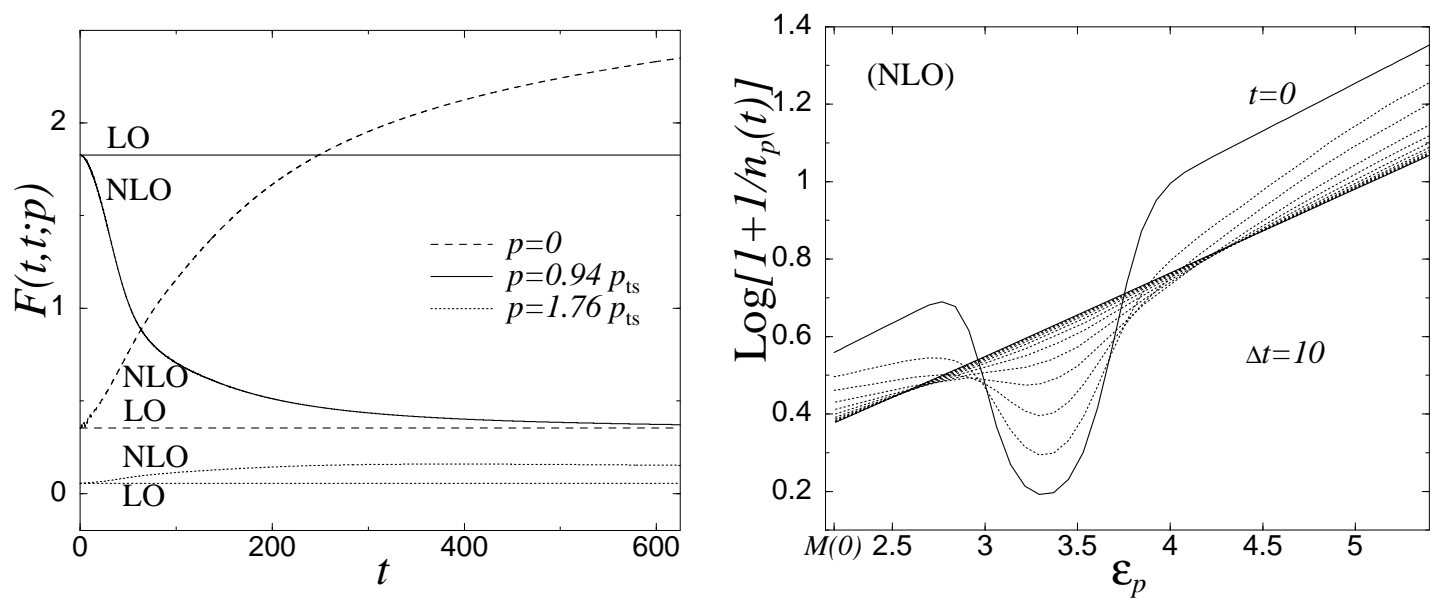

FIGURE 3. LEFT: Comparison of the LO and NLO time dependence of the equal-time correlation modes $F(t, t ; p)$ for the "tsunami" initial condition (197)-(199). The importance of scattering included in the NLO approximation is apparent: the non-thermal LO fixed points become unstable and the "tsunami" decays, approaching thermal equilibrium at late times. RIGHT: Effective particle number distribution for a "tsunami" in the presence of a thermal background. The solid line shows the initial distribution which for low and for high momenta follows a Bose-Einstein distribution, i.e. $\log \left[1+1 / n_{p}(0)\right] \simeq \varepsilon_{p}(0) / T_{0}$. At late times the non-thermal distribution equilibrates and approaches a straight line with inverse slope $T_{\mathrm{eq}}>T_{0}$.

change in the effective mass term drives the system out of equilibrium and one can study its relaxation. We present $M^{2}(t)$ for three different couplings $\lambda=\lambda_{0} \equiv 0.5 M^{2}(0)$ (bottom), $\lambda=10 \lambda_{0}$ (middle) and $\lambda=40 \lambda_{0}$ (top). All quantities are given in units of appropriate powers of the initial-time mass $M(0)$. Therefore, all curves in the left graph of Fig. 4 start at one. The time-dependent mass squared $M^{2}(t)$ shoots up in response to the "quench" and stays below the value $2 M(0)^{2}$ of the initial thermal distribution. The amplitude of initial oscillations is quickly reduced and, averaged over the oscillation time-scale, the evolution is rapidly driven towards the asymptotic values.

We compare the asymptotic values with the self-consistent solution of the LO mass equation (196) for constant mass squared $M_{\mathrm{gap}}^{2}$ and given particle number distribution $n_{p}(0):{ }^{16}$

$$
M_{\text {gap }}^{2}=m^{2}+\frac{\lambda}{6} \int \frac{\mathrm{d} p}{2 \pi}\left(n_{p}(0)+\frac{1}{2}\right) \frac{1}{\sqrt{p^{2}+M_{\text {gap }}^{2}}} .
$$

The result from this gap equation is $M_{\text {gap }}^{2}=\{1.01,1.10,1.29\} M^{2}(0)$ for the three values of $\lambda$, respectively. For this wide range of couplings the values are in good numerical agreement with the corresponding dynamical large-time results inferred from Fig. 4 as $\{1.01,1.11,1.31\} M^{2}(0)$ at $t=200 / M(0)$. We explicitly checked that at $t=400 / M(0)$

${ }^{16}$ Here the logarithmic divergence of the one-dimensional integral is absorbed into the bare mass parameter $m^{2}$ using the same renormalization condition as for the dynamical evolution in the LO approximation, i.e. $m^{2}+\frac{\lambda}{6} \int \frac{\mathrm{d} p}{2 \pi}\left(n_{p}(0)+\frac{1}{2}\right)\left(p^{2}+M^{2}(0)\right)^{-1 / 2}=M^{2}(0)$. 

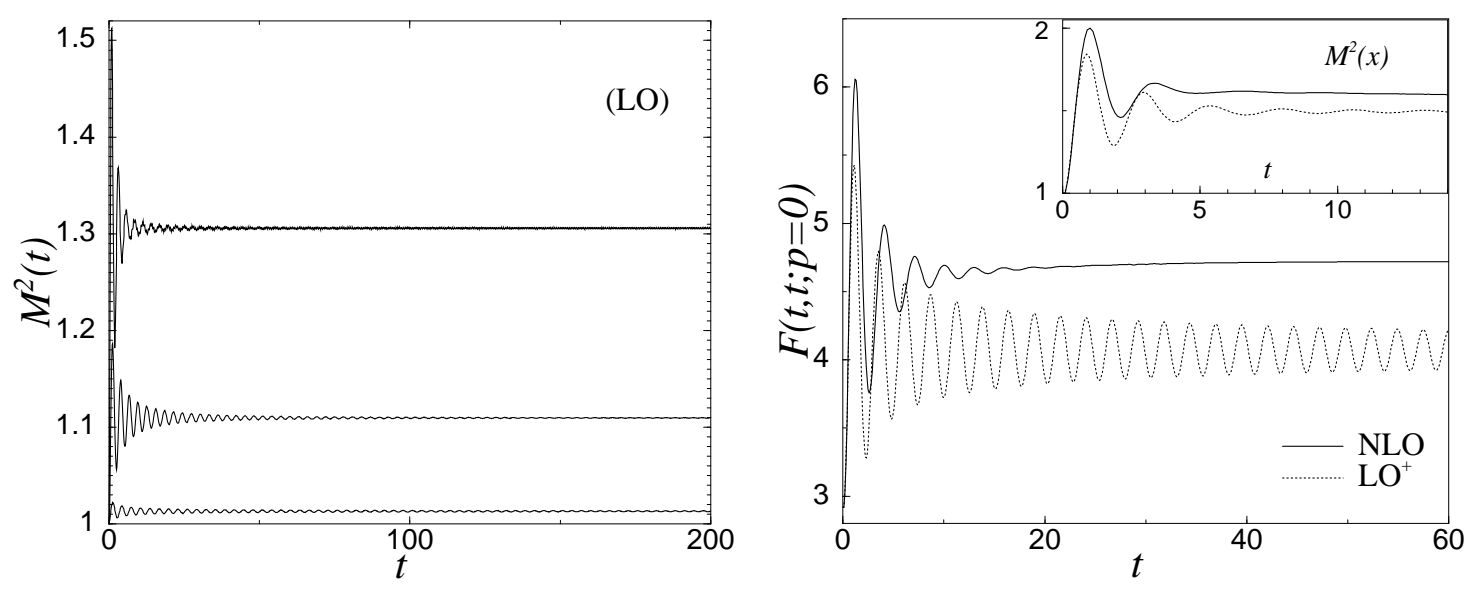

FIGURE 4. LEFT: Shown is the time-dependent mass term $M^{2}(t)$ in the LO approximation for three different couplings following a "quench". All quantities are given in units of appropriate powers of the initial-time mass $M(0)$. RIGHT: Time dependence of the equal-time zero-mode $F(t, t ; p=0)$ after a "quench" (see text for details). The inset shows the mass term $M^{2}(t)$, which includes a sum over all modes. The dotted lines represent the Hartree approximation $\left(\mathrm{LO}^{+}\right)$, while the solid lines give the NLO results. The coupling is $\lambda / 6 N=0.17 M^{2}(0)$ for $N=4$.

these values are still the same. One concludes that the asymptotic behavior at LO is well described in terms of the initial particle number distribution $n_{p}(0)$. The latter is not a thermal distribution for the late-time mass terms with values smaller than $2 M^{2}(0)$.

The LO approximation for $F\left(t, t^{\prime} ; p\right)$ and $\rho\left(t, t^{\prime} ; p\right)$ becomes exact ${ }^{17}$ for $t, t^{\prime} \rightarrow 0$, since the memory integrals on the r.h.s. of the exact Eqs. (138) vanish at initial time. For very early times one therefore expects the LO approximation to yield a quantitatively valid description. However, from Fig. 4 one observes that the time evolution is dominated already at early times by the non-thermal LO fixed points. As discussed above, the latter are artefacts of the approximation. Though the precise numerical values of the LO fixed points are pure artefacts, we emphasize that the presence of approximate fixed points governing the early-time behavior is a qualitative feature that can be observed also beyond LO (cf. below). The question of how strongly the LO late-time results deviate from thermal equilibrium depends of course crucially on the details of the initial conditions. Typically, time- and/or momentum-averaged quantities are better determined by the LO approximation than quantities characterizing a specific momentum mode. This is exemplified on the right of Fig. 4, which shows the equal-time zero-mode $F(t, t ; p=0)$ along with $M^{2}(t)$ including the sum over all modes. Here we employ a "quench" with a larger drop in the effective mass term from $2.9 M^{2}(0)$ to $M^{2}(0)$. The initial particle number distribution is $n_{p}(0)=1 /\left(\exp \left[\sqrt{p^{2}+M^{2}(0)} / T_{0}\right]-1\right)$ with $T_{0}=8.5 M(0)$. In the figure the dotted curves show the dynamics obtained from an "improved" LO (Hartree) approximation, $\mathrm{LO}^{+}$, that takes into account the local part of the NLO self-energy contribution. The resulting equations have the very same structure

\footnotetext{
17 This is due to the fact that we choose to consider Gaussian initial conditions.
} 

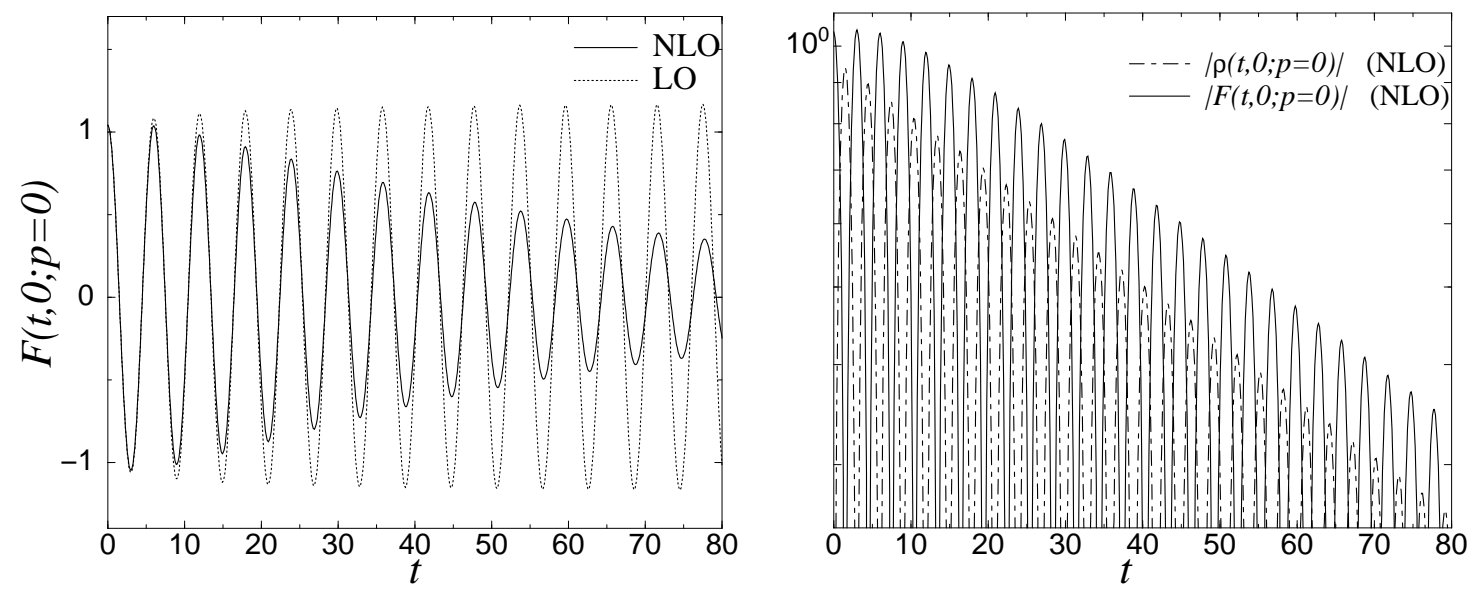

FIGURE 5. LEFT: Shown is the evolution of the unequal-time correlation $F(t, 0 ; p=0)$ after a "quench". Unequal-time correlation functions approach zero in the NLO approximation and correlations with early times are effectively suppressed $\left(\lambda / 6 N=(5 / 6 N \simeq 0.083) M^{2}(0)\right.$ for $\left.N=10\right)$. In contrast, there is no decay of correlations with earlier times for the LO approximation. RIGHT: The logarithmic plot of $|\rho(t, 0 ; p=0)|$ and $|F(t, 0 ; p=0)|$ as a function of time $t$ shows an oscillation envelope which quickly approaches a straight line. At NLO the correlation modes therefore approach an exponentially damped behavior. (All in units of $M(0)$.)

as the LO ones, however, with the LO and NLO contribution to the mass term $M^{2}(t)$ included as given by Eq. (178) below. The large-time limit of the mass term in the $\mathrm{LO}^{+}$ approximation is determined by the $\mathrm{LO}^{+}$fixed point solution in complete analogy to the above discussion. We also give in Fig. 4 the NLO results, which are discussed below.

The effective loss of details about the initial conditions is a prerequisite for thermalization. At LO this is obstructed by an infinite number of spurious conserved quantities (mode particle numbers), which keep initial-time information. An important quantity in this context is the unequal-time two-point function $F(t, 0 ; p)$, which characterizes the correlations with the initial time. Clearly, if thermal equilibrium is approached then these correlations should be damped. On the left of Fig. 5 the dotted line shows the unequaltime zero-mode $F(t, 0 ; p=0)$ following the same "quench" at LO as for Fig. 4 left. One observes no decay of correlations with earlier times for the LO approximation. Scattering effects entering at NLO are crucial for a sufficient effective loss of memory about the initial conditions, which is discussed next.

\subsubsection{NLO thermalization}

In contrast to the LO approximation at NLO the self-energies $\Sigma_{F}$ and $\Sigma_{\rho} \sim \mathscr{O}(1 / N)$ do not vanish. For the initial conditions (197) all correlators are diagonal in field index space and $\phi \equiv 0$ for all times. In this case the evolution equations derived from the NLO 2PI effective action (79) are given by (138) with the self-energies (178)-(182). As discussed in Sec. 3.4.3, the NLO evolution equations are causal equations with characteristic "memory" integrals, which integrate over the time history of the evolution 
taken to start at time $t_{0}=0$ without loss of generality.

We consider first the same "tsunami" initial condition (197) as for the LO case discussed above. The result is shown on the left of Fig. 3 for $N=10$. One observes a dramatic effect of the NLO corrections! They quickly lead to a decay of the initially high population of modes around $p_{\mathrm{ts}}$. On the other hand, low momentum modes get populated such that thermal equilibrium is approached at late times. In order to make this apparent one can plot the results in a different way. For this we note that according to (197) the statistical propagator corresponds to the ratio of the following particle number at initial time $t=0$ :

$$
n_{\mathbf{p}}(t)+\frac{1}{2}=\left[F(t, t ; \mathbf{p}) K(t, t ; \mathbf{p})-Q^{2}(t, t ; \mathbf{p})\right]^{1 / 2},
$$

and the corresponding mode energy. Here we have defined:

$$
K\left(t, t^{\prime} ; \mathbf{p}\right) \equiv \partial_{t} \partial_{t^{\prime}} F\left(t, t^{\prime} ; \mathbf{p}\right) \quad, \quad Q\left(t, t^{\prime} ; \mathbf{p}\right) \equiv \frac{1}{2}\left[\partial_{t} F\left(t, t^{\prime} ; \mathbf{p}\right)+\partial_{t^{\prime}} F\left(t, t^{\prime} ; \mathbf{p}\right)\right]
$$

Since we employed $Q(0,0 ; \mathbf{p})=0$ for the initial conditions (197) the initial mode energy is given by

$$
\varepsilon_{\mathbf{p}}(t)=\left(\frac{K(t, t ; \mathbf{p})}{F(t, t ; \mathbf{p})}\right)^{1 / 2},
$$

such that $F(t, t ; p)=\left[n_{\mathbf{p}}(t)+1 / 2\right] / \varepsilon_{\mathbf{p}}(t)$. For illustration of the results we may use (201) and (203) for times $t>0$ in order to define an effective mode particle number and energy, where we set $Q\left(t, t^{\prime} ; \mathbf{p}\right) \equiv 0$ in (201) for the moment. The behavior of the effective particle number is illustrated on the right of Fig. 3, where we plot $\log (1+$ $\left.1 / n_{\mathbf{p}}(t)\right)$ as a function of $\varepsilon_{\mathbf{p}}(t)$. Note that for a Bose-Einstein distributed effective particle number this is proportional to the inverse temperature: $\log \left(1+1 / n_{\mathrm{BE}}(T)\right) \sim 1 / T$. For the corresponding plot of Fig. 3 we have employed an initial "tsunami" at $p_{\text {ts }} / m_{R}=$ 2.5, where we added an initial "thermal background" distribution with temperature $T_{0} / m_{R}=4 .{ }^{18}$ Correspondingly, from the solid line $(t=0)$ in the right figure one observes the initial "thermal background" as a straight line distorted by the non-thermal "tsunami" peak. The curves represent snapshots at equidistant time steps $\Delta t m_{R}=10$. After rapid changes in $n_{p}(t)$ at early times the subsequent curves converge to a straight line to high accuracy, with inverse slope $T_{\mathrm{eq}} / T_{0}=1.175$. The initial high occupation number in a small momentum range decays quickly with time. More and more low momentum modes get populated and the particle distribution approaches a thermal shape.

The crucial importance of the NLO corrections for the nonequilibrium dynamics can also be observed for other initial conditions. The right graph of Fig. 4 shows the equaltime zero-mode $F(t, t ; p=0)$, along with $M^{2}(t)$ including the sum over all modes, following a "quench" as described in Sec. 4.1.1. While the dynamics for vanishing selfenergies $\Sigma_{F}$ and $\Sigma_{\rho}$ is quickly dominated by the spurious LO fixed points, this is no longer the case once the NLO self-energy corrections are included. In particular, one observes a very efficient damping of oscillations at NLO. This becomes even more pronounced for unequal-time correlators as shown for $F(t, 0 ; p=0)$ in the left graph of

18 The initial mass term is $M(0) / m_{R}=2.24$ and $\lambda / 6 N=0.5 m_{R}^{2}$ for $N=4$. 
Fig. 5. We find that the unequal-time two-point functions approach zero for the NLO approximation and correlations with early times are effectively suppressed. Of course, time-reversal invariance implies that the oscillations can never be damped out to zero completely during the nonequilibrium time evolution, however, zero can be approached arbitrarily closely. In the NLO approximation we find that all modes $F\left(t, t^{\prime} ; p\right)$ and $\rho\left(t, t^{\prime} ; p\right)$ approach an approximately exponential damping behavior for both equal-time and unequal-time correlations. On the right of Fig. 5 the approach to an exponential behavior is demonstrated for $|\rho(t, 0 ; p=0)|$ and $|F(t, 0 ; p=0)|$ with the same parameters as for the left figure. The logarithmic plot shows that after a non-exponential period at early times the envelope of oscillations can be well approximated by a straight line. From an asymptotic envelope fit of $F(t, 0 ; p=0)$ to an exponential form $\sim \exp \left(-\gamma_{0}^{(\text {damp })} t\right)$ we obtain here a damping rate $\gamma_{0}^{(\mathrm{damp})}=0.016 M(0)$. For comparison, for the parameters employed for the right graph of Fig. 4 we find $\gamma_{0}^{(\text {damp })}=0.11 M(0)$ from an exponential fit to the asymptotic behavior of $F(t, 0 ; p=0)$. The oscillation frequency of $F(t, 0 ; p=0)$ is found to quickly stabilize around $1.1 M(0) / 2 \pi \simeq 0.18 M(0)$, which is of the same order than the damping rate. Correspondingly, one observes in Fig. 4 an equal-time zero mode which is effectively damped out at NLO after a few oscillations. The oscillation frequency is always found to stabilize very quickly and to be quantitatively well described by $\varepsilon_{\mathbf{p}}(t) / 2 \pi$ for the unequal-time modes $\left(\varepsilon_{\mathbf{p}}(t) / \pi\right.$ for the equal-time modes) with the effective mode energy $\varepsilon_{\mathbf{p}}(t)$ given by (203). We emphasize that the parameter $M(t)$ given by (178) cannot be used in general to characterize well the oscillation frequencies of the correlation zero-modes. Beyond LO or Hartree the renormalized mass, which can be obtained from the zero-mode oscillation frequency, receives corrections from the non-vanishing self-energies $\Sigma_{F}$ and $\Sigma_{\rho}$.

The strong qualitative difference between LO and NLO appears because an infinite number of spurious conserved quantities is removed once scattering is taken into account. It should be emphasized that the step going from LO to NLO is qualitatively very different than the one going from NLO to NNLO or further. In order to understand better what happens going from LO to NLO we consider again the effective particle number (201). It is straightforward by taking the time derivative on both sides of (201) to obtain an exact evolution equation for $n_{\mathbf{p}}(t)$ with the help of the exact relations (138):

$$
\begin{aligned}
\left(n_{\mathbf{p}}(t)+\frac{1}{2}\right) \partial_{t} n_{\mathbf{p}}(t)= & \\
\int_{t_{0}}^{t} d t^{\prime \prime} & \left\{\left.\left[\Sigma_{\rho}\left(t, t^{\prime \prime} ; \mathbf{p}\right) F\left(t^{\prime \prime}, t ; \mathbf{p}\right)-\Sigma_{F}\left(t, t^{\prime \prime} ; \mathbf{p}\right) \rho\left(t^{\prime \prime}, t ; \mathbf{p}\right)\right] \partial_{t} F\left(t, t^{\prime} ; \mathbf{p}\right)\right|_{t=t^{\prime}}\right. \\
& \left.-\left[\Sigma_{\rho}\left(t, t^{\prime \prime} ; \mathbf{p}\right) \partial_{t} F\left(t^{\prime \prime}, t ; \mathbf{p}\right)-\Sigma_{F}\left(t, t^{\prime \prime} ; \mathbf{p}\right) \partial_{t} \rho\left(t^{\prime \prime}, t ; \mathbf{p}\right)\right] F(t, t ; \mathbf{p})\right\}
\end{aligned}
$$

Here $t_{0}$ denotes the initial time which was set to zero in (138) without loss of generality. Since $\Sigma_{F} \sim \mathscr{O}(1 / N)$ as well as $\Sigma_{\rho}$, one directly observes that at LO, i.e. for $N \rightarrow \infty$, the particle number for each momentum mode is strictly conserved: $\partial_{t} n_{\mathbf{p}}(t) \equiv 0$ at LO. Stated differently, eq. (201) just specifies the infinite number of additional constants of motion which appear at LO. In contrast, once corrections beyond LO are taken 
into account then (201) no longer represent conserved quantities. The first non-zero contribution to the self-energies occurs at NLO. As a consequence $\partial_{t} n_{\mathbf{p}}(t) \not \equiv 0$ in general.

For the LO approximation we have seen that there is an infinite number of timetranslation invariant solutions (201) to the nonequilibrium evolution equations. Thermal equilibrium plays no particular role at LO. However, this is very different at NLO and beyond. It is very instructive to consider what is required to find solutions of (204) which are homogeneous in time. Of course, any time-translation invariant solution cannot be achieved if one respects all symmetries of the quantum field theory such as time reflection symmetry. However, we can consider the limit $t_{0} \rightarrow-\infty$ and ask under what conditions time-translation invariant $F\left(t, t^{\prime} ; \mathbf{p}\right)=F^{(\text {hom })}\left(t-t^{\prime} ; \mathbf{p}\right)$ etc. represent solutions of (204). Using the (anti-)symmetry of the (spectral function) statistical propagator we have

$$
\begin{aligned}
& \rho^{(\text {hom })}\left(t-t^{\prime} ; \mathbf{p}\right)=-i \int \frac{\mathrm{d} \omega}{2 \pi} \sin \left[\omega\left(t-t^{\prime}\right)\right] \rho^{(\text {hom })}(\omega, \mathbf{p}) \\
& F^{(\text {hom })}\left(t-t^{\prime} ; \mathbf{p}\right)=\int \frac{\mathrm{d} \omega}{2 \pi} \cos \left[\omega\left(t-t^{\prime}\right)\right] F^{(\text {hom })}(\omega, \mathbf{p}) .
\end{aligned}
$$

Note that in our conventions $\rho^{(\mathrm{hom})}(\omega ; \mathbf{p})=-\rho^{(\mathrm{hom})}(-\omega ; \mathbf{p})$ is purely imaginary, while $F^{(\text {hom })}(\omega ; \mathbf{p})=F^{(\text {hom })}(-\omega ; \mathbf{p})$ is real. Since $\left.\partial_{t} F^{(\text {hom })}\left(t-t^{\prime} ; \mathbf{p}\right)\right|_{t=t^{\prime}} \equiv 0$, time-translation invariant solutions of (204) require:

$$
\begin{aligned}
\lim _{\left(t-t_{0}\right) \rightarrow \infty} \int_{t_{0}}^{t} d t^{\prime \prime} & {\left[\Sigma_{\rho}^{(\text {hom })}\left(t-t^{\prime \prime} ; \mathbf{p}\right) \partial_{t} F^{(\text {hom })}\left(t^{\prime \prime}-t ; \mathbf{p}\right)\right.} \\
& \left.-\Sigma_{F}^{(\text {hom })}\left(t-t^{\prime \prime} ; \mathbf{p}\right) \partial_{t} \rho^{(\text {hom })}\left(t^{\prime \prime}-t ; \mathbf{p}\right)\right]=0 .
\end{aligned}
$$

Inserting the Fourier transforms (205) and the respective ones for the self-energies and using

$$
\lim _{\left(t-t_{0}\right) \rightarrow \infty} \frac{\sin \left[\omega\left(t-t_{0}\right)\right]}{\omega}=\pi \delta(\omega),
$$

one finds that the condition (206) can be equivalently written as

$$
\int \mathrm{d} \omega \omega\left[\Sigma_{\rho}^{(\mathrm{hom})}(\omega, \mathbf{p}) F^{(\mathrm{hom})}(\omega, \mathbf{p})-\Sigma_{F}^{(\mathrm{hom})}(\omega, \mathbf{p}) \rho^{(\mathrm{hom})}(\omega, \mathbf{p})\right]=0 .
$$

In contrast to the LO approximation, at NLO and beyond we find with $\Sigma^{(\text {hom })} \neq 0$ that time-translation invariant solutions are highly nontrivial. However, one observes from the equilibrium fluctuation-dissipation relation (129) with (130) that indeed thermal equilibrium singles out a solution which fulfills this condition. We emphasize that in equilibrium the fluctuation-dissipation relation is fulfilled at all orders in the $2 \mathrm{PI} 1 / \mathrm{N}$ expansion. In this respect there is no qualitative change going from NLO to NNLO or beyond. In particular, very similar results as shown in Fig. 3 are obtained from the loop expansion of the 2PI effective action beyond two-loop order, which is demonstrated in Sec. 4.1.4. Three-loop is required since the two-loop order corresponds to a Gaussian approximation, which suffers from the same infinite number of spurious conserved quantities as the LO approximation. 
We emphasize that the above discussion does not imply that the evolution always has to approach thermal equilibrium at late times. There are many initial conditions for which the system cannot thermalize. Obvious examples are initial conditions inferred from statistical ensembles which do not obey the clustering property. These do not exhibit thermal correlation functions in general.

\subsubsection{Detour: Boltzmann equation}

The nonequilibrium 2PI effective action can be employed to obtain effective kinetic or Boltzmann-type descriptions for quasiparticle number distributions. The latter are widely used in the literature and it is important to understand their merits and limits. The "derivation" of such an equation from the 2 PI effective action requires a number of additional approximations which go beyond a loop or $1 / N$ expansion of the 2PI effective action. At the end of this procedure we will find an irreversible equation, which therefore lost part of the symmetries of the underlying quantum field theory. The comparison will allow us, in particular, to clearly point out the role of neglected off-shell effects as compared to the quantum field theory description.

Kinetic descriptions concentrate on the behavior of particle number distributions. The latter contain information about the statistical propagator $F(x, y)$ at equal times $x^{0}=y^{0}$. Correlations between unequal times are outside the scope of a Boltzmann equation. We can therefore start the discussion from the exact equation for the effective particle number (204). ${ }^{19}$ For our current purposes it is irrelevant that we will consider a spatially homogeneous one-component field theory with vanishing field expectation value $(\phi=0)$. The steps leading to the Boltzmann equation are summarized as follows:

1. Consider a 2 PI loop expansion of $\Gamma[G]$ to three-loop order. This leads to self-energy corrections up to two loops, diagrammatically given by $\bigcirc, \cdots$, which lead to the time-dependent mass term

$$
M^{2}(t)=m^{2}+\frac{\lambda}{2} \int_{\mathbf{p}} F(t, t ; \mathbf{p})
$$

and

$\Sigma_{F}\left(t, t^{\prime} ; \mathbf{p}\right)=-\frac{\lambda^{2}}{6} \int_{\mathbf{q}, \mathbf{k}} F\left(t, t^{\prime} ; \mathbf{p}-\mathbf{q}-\mathbf{k}\right)\left[F\left(t, t^{\prime} ; \mathbf{q}\right) F\left(t, t^{\prime} ; \mathbf{k}\right)-\frac{3}{4} \rho\left(t, t^{\prime} ; \mathbf{q}\right) \rho\left(t, t^{\prime} ; \mathbf{k}\right)\right]$,
$\Sigma_{\rho}\left(t, t^{\prime} ; \mathbf{p}\right)=-\frac{\lambda^{2}}{2} \int_{\mathbf{q}, \mathbf{k}} \rho\left(t, t^{\prime} ; \mathbf{p}-\mathbf{q}-\mathbf{k}\right)\left[F\left(t, t^{\prime} ; \mathbf{q}\right) F\left(t, t^{\prime} ; \mathbf{k}\right)-\frac{1}{12} \rho\left(t, t^{\prime} ; \mathbf{q}\right) \rho\left(t, t^{\prime} ; \mathbf{k}\right)\right]$.

19 For this it is useful to rewrite the following characteristic term as:

$$
-i\left(\Sigma_{\rho} F-\Sigma_{F} \rho\right)=\left(\Sigma_{F}-i \Sigma_{\rho} / 2\right)(F+i \rho / 2)-\left(\Sigma_{F}+i \Sigma_{\rho} / 2\right)(F-i \rho / 2) .
$$

The difference of the two terms on the r.h.s. can be directly interpreted as the difference of a "loss" and a "gain" term in a Boltzmann type description. 
2. Choose a quasiparticle ansatz, i.e. a free-field type form for the two-point functions and their derivative. This corresponds to the replacements on the r.h.s. of (204):

$$
\begin{aligned}
& F\left(t^{\prime \prime}, t ; \mathbf{p}\right) \rightarrow\left(n_{\mathbf{p}}+1 / 2\right) \cos \left[\omega_{\mathbf{p}}\left(t^{\prime \prime}-t\right)\right] / \omega_{\mathbf{p}}, \\
& \rho\left(t^{\prime \prime}, t ; \mathbf{p}\right) \rightarrow \sin \left[\omega_{\mathbf{p}}\left(t^{\prime \prime}-t\right)\right] / \omega_{\mathbf{p}}, \\
& \partial_{t} F\left(t^{\prime \prime}, t ; \mathbf{p}\right) \rightarrow\left(n_{\mathbf{p}}+1 / 2\right) \sin \left[\omega_{\mathbf{p}}\left(t^{\prime \prime}-t\right)\right], \\
& \partial_{t} \rho\left(t^{\prime \prime}, t ; \mathbf{p}\right) \rightarrow-\cos \left[\omega_{\mathbf{p}}\left(t^{\prime \prime}-t\right)\right] \text {. }
\end{aligned}
$$

3. Here the particle number $n_{\mathbf{p}}$ and mode energy $\omega_{\mathbf{p}}$ may still depend weakly on time: One assumes a separation of scales, with a sufficiently slow time variation of $n_{\mathbf{p}}(t)$ such that one can pull all factors of $n_{\mathbf{p}}(t)$ out of the time integral on the r.h.s. of (204). The particle numbers are then evaluated at the latest time of the memory integral.

4. Send the initial time $t_{0}$ to the remote past: $t_{0} \rightarrow-\infty$. Of course, by construction the resulting equation is not meant to describe the detailed early-time behavior since $t_{0} \rightarrow-\infty$. In the context of kinetic descriptions, one finally specifies the initial condition for the effective particle number distribution at some finite time and approximates the evolution by the equation with $t_{0}$ in the remote past.

A standard alternative derivation employs first steps 1. and 4., then a first-order gradient expansion of the two-point functions in the center coordinate $\left(t+t^{\prime}\right) / 2$ and then a quasiparticle ansatz. However, for the typically employed first-order gradient approximation both approaches are fully equivalent. The current procedure has the advantage that one can send the initial time $t_{0} \rightarrow-\infty$ last, which allows one to discuss a few finite-time effects that are typically discarded. Applying the assumptions 1.-3. to the exact equation (204) leads after some lengthy shuffling of terms to the expression:

$$
\begin{aligned}
\partial_{t} n_{\mathbf{p}}(t)=\frac{\lambda^{2}}{3} \int_{\mathbf{s q} \mathbf{k}}(2 \pi)^{d} \delta(\mathbf{p}-\mathbf{q}-\mathbf{k}-\mathbf{s}) \frac{1}{2 \omega_{\mathbf{p}} 2 \omega_{\mathbf{q}} 2 \omega_{\mathbf{k}} 2 \omega_{\mathbf{s}}} \\
\quad\left\{\left[\left(1+n_{\mathbf{p}}\right)\left(1+n_{\mathbf{q}}\right)\left(1+n_{\mathbf{k}}\right)\left(1+n_{\mathbf{s}}\right)-n_{\mathbf{p}} n_{\mathbf{q}} n_{\mathbf{k}} n_{\mathbf{s}}\right]\right. \\
+\quad \int_{t_{0}}^{t} d t^{\prime \prime} \cos \left[\left(\omega_{\mathbf{p}}+\omega_{\mathbf{q}}+\omega_{\mathbf{k}}+\omega_{\mathbf{s}}\right)\left(t-t^{\prime \prime}\right)\right] \\
+\quad 3\left[\left(1+n_{\mathbf{p}}\right)\left(1+n_{\mathbf{q}}\right)\left(1+n_{\mathbf{k}}\right) n_{\mathbf{s}}-n_{\mathbf{p}} n_{\mathbf{q}} n_{\mathbf{k}}\left(1+n_{\mathbf{s}}\right)\right] \\
+\quad 3\left[\left(1+n_{\mathbf{p}}\right)\left(1+n_{\mathbf{q}}\right) n_{\mathbf{k}} n_{\mathbf{s}}-n_{\mathbf{p}} n_{\mathbf{q}}\left(1+n_{\mathbf{k}}\right)\left(1+n_{\mathbf{s}}\right)\right] \\
\quad \int_{t_{0}}^{t} d t^{\prime \prime} \cos \left[\left(\omega_{\mathbf{p}}+\omega_{\mathbf{q}}+\omega_{\mathbf{k}}-\omega_{\mathbf{s}}\right)\left(t-t^{\prime \prime}\right)\right] \\
+\quad\left[\left(1+n_{\mathbf{p}}\right) n_{\mathbf{q}} n_{\mathbf{k}} n_{\mathbf{s}}-n_{\mathbf{p}}\left(1+n_{\mathbf{q}}\right)\left(1+n_{\mathbf{k}}\right)\left(1+n_{\mathbf{s}}\right)\right] \\
\left.\quad \int_{t_{0}}^{t} d t^{\prime \prime} \cos \left[\left(\omega_{\mathbf{p}}-\omega_{\mathbf{q}}-\omega_{\mathbf{k}}-\omega_{\mathbf{s}}\right)\left(t-t^{\prime \prime}\right)\right]\right\} .
\end{aligned}
$$


The contributions on the r.h.s. of this equation have a typical "gain \& loss" structure with a simple interpretation:

(I) describes production and annihilation of four "quasiparticles" $(0 \rightarrow 4,4 \rightarrow 0)$.

(II) and (IV) describe $1 \rightarrow 3$ and $3 \rightarrow 1$ processes.

(III) describes $2 \leftrightarrow 2$ scattering processes, which are the well-known contributions to the standard Boltzmann equation.

We emphasize that only the processes described by (III) lead to a non-zero contribution in the limit $\left(t-t_{0}\right) \rightarrow \infty$. For time-independent mode energies $\omega_{\mathbf{p}}$ one can perform the time integrations in (213) explicitly. This leads using (207) to $\delta$-functions, which enforce the strict conservation of the mode energies involved in the scattering. At this point the description of the quantum field theory has been fully reduced to the physics of colliding "on-shell" quasiparticles. Of course, strict energy and momentum conservation for these quasiparticles leads to vanishing contributions (I), (II) and (IV). The latter describe processes, which change the total particle number such as $1 \rightarrow 3$ processes. We will see below that in a quantum field theory "off-shell" particle number changing processes such as described by (II) can play a very important role! An obvious example is given by the fact that the Boltzmann equation including the contribution (III) admits grand canonical thermal solutions with nonzero chemical potential $\mu$ :

$$
n_{\mathbf{p}}(t) \rightarrow \frac{1}{e^{\left(\omega_{\mathbf{p}}-\mu\right) / T}-1}
$$

However, the real scalar quantum field theory is charge neutral and $\mu \equiv 0$ ! We conclude that taking into account only $2 \leftrightarrow 2$ scattering processes of "on-shell" quasiparticles obviously fails to describe the quantum world if for a given initial condition the chemical potential turns out to be large. We emphasize that total particle number changing processes are included in the original quantum field theory equation before applying the additional steps 2.-4. In particular, they do not only appear as contributions from finite initial-time effects as discussed here for illustration. The main "off-shell" effects are removed in step 2., when the quasiparticle assumption enters the description.

The physics of "off-shell" processes can be very nicely observed in the $1+1$ dimensional quantum field theory. Note that for one spatial dimension the contribution (III) vanishes as well in the limit $\left(t-t_{0}\right) \rightarrow \infty$ ! In this case both momentum and energy conservation, $p+q-k-s=0 \wedge \sqrt{p^{2}+M^{2}}+\sqrt{q^{2}+M^{2}}-\sqrt{k^{2}+M^{2}}-\sqrt{s^{2}+M^{2}}=0$, lead to an "ineffective" two-to-two scattering where the incoming and outcoming modes are unaffected: for instance with $p=k$ and $q=s$ the contribution (III) in Eq. (213) vanishes identically. The equation becomes trivial in this case. We conclude that for the $1+1$ dimensional quantum field theory there is no dynamics from the Boltzmann equation! In contrast, from the same three-loop approximation of the 2PI effective action without the steps 2.-4. we will see in the next section that the $1+1$ dimensional quantum field theory thermalizes. 

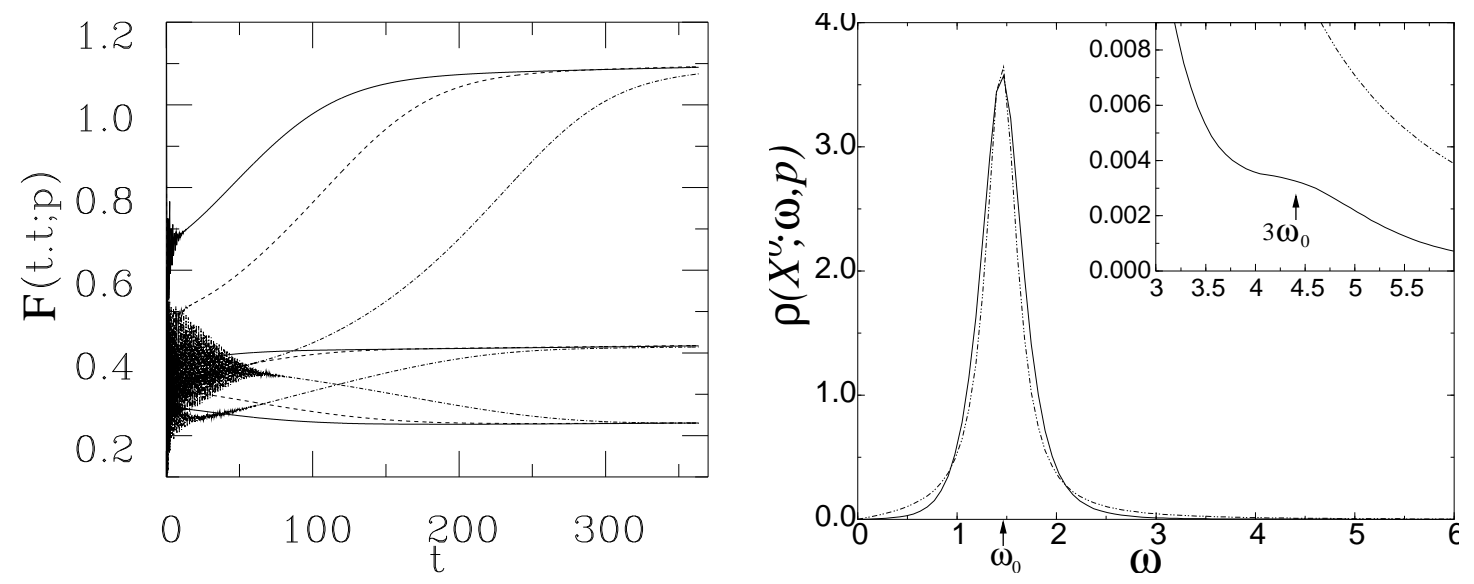

FIGURE 6. LEFT: Examples for the time dependence of the equal-time propagator $F(t, t ; \mathbf{p})$ with Fourier modes $p=0,3,5$ from the 2PI three-loop effective action. The evolution is shown for three very different nonequilibrium initial conditions with the same energy density (all in initial-mass units). RIGHT: Wigner transform $\rho\left(X^{0} ; \omega, p\right)$ of the spectral function as a function of $\omega$ at $X^{0}=35.1$ for $p=0$ (in units of $\left.m_{R}\right)$. Also shown are fits to a Breit-Wigner function (dotted) with $\left(\omega_{0}, \Gamma_{0}\right)=(1.46,0.37)$. The inset shows a blow-up around $3 \omega_{0}$. The expected bump from "off-shell" $1 \leftrightarrow 3$ processes is small but visible.

\subsubsection{Characteristic time scales}

Nonequilibrium dynamics requires the specification of an initial state. A crucial question of thermalization is how quickly the nonequilibrium system effectively looses the details about the initial conditions, and what are the characteristic stages of a partial loss of information. Thermal equilibrium keeps no memory about the time history except for the values of a few conserved charges. As a consequence, for the real scalar field theory thermalization requires that the late-time result is uniquely determined by energy density.

In Fig. 6 we show the time dependence of the equal-time propagator $F(t, t ; \mathbf{p})$ for three Fourier modes $p=0,3,5$ and three very different initial conditions with the same energy density. All quantities are given in appropriate units of the mass at initial time, and we consider $1+1$ dimensions. For the solid line the initial conditions are close to a mean field thermal solution, the initial mode distribution for the dashed and the dashed-dotted lines deviate more and more substantially from a thermal equilibrium distribution. It is striking to observe that propagator modes with very different initial values but with the same momentum $p$ approach the same large-time value. The asymptotic behavior of the two-point function modes are universal and uniquely determined by the initial energy density.

One observes that after an effective damping of rapid oscillations the modes are still far from equilibrium. All correlation functions quickly approach an exponentially damped behavior. A characteristic rate $\gamma_{0}^{(\mathrm{damp})}$ can be obtained from the zero mode of the unequal-time two-point function $F(t, 0 ; p=0)$ with a corresponding time scale proportional to the inverse rate. In this early-time range correlations with the initial time are effectively suppressed and asymptotically $F(t, 0 ; p=0) \rightarrow 0^{+}$. The early- 
time range is followed by a smooth "drifting" of modes, which is characterized by a slow dependence of $F\left(t, t^{\prime} ; p=0\right)$ on $\left(t+t^{\prime}\right) / 2$. The presence of such a regime is a prerequisite for descriptions based on gradient expansions in the center coordinate $\left(t+t^{\prime}\right) / 2$. Clearly, the early-time behavior for times $\sim 1 / \gamma_{0}^{(\mathrm{damp})}$ is beyond the scope of a gradient expansion. Though the exponential damping at early times is crucial for an effective loss of details of the initial conditions, it does not determine the time scale for thermalization. One typically finds very different rates for damping and for the late-time approach of $F(t, t ; p=0)$ to thermal equilibrium.

One of the "key" properties for the success of the quantum field theoretical description is the nontrivial (i.e. not the free-field or " $\delta$ "-type) dynamical spectral function $\rho$. To analyze the spectral function (not to solve the dynamics!), we perform a Wigner transformation for the modes $\rho\left(t, t^{\prime} ; \mathbf{p}\right)$ and write with $X^{0}=\left(t+t^{\prime}\right) / 2, s^{0}=t-t^{\prime}$ :

$$
i \rho\left(X^{0} ; \omega, p\right)=\int_{-2 X^{0}}^{2 X^{0}} d s^{0} e^{i \omega s^{0}} \rho\left(X^{0}+s^{0} / 2, X^{0}-s^{0} / 2 ; p\right) .
$$

The $i$ is introduced such that $\rho\left(X^{0} ; \omega, \mathbf{p}\right)$ is real. Because the spectral function is antisymmetric, $\rho\left(X^{0} ; \omega, \mathbf{p}\right)=-\rho\left(X^{0} ;-\omega, \mathbf{p}\right)$, and we will present the positive-frequency part only. Since we consider an initial-value problem with $t, t^{\prime} \geq 0$, the time integral over $s^{0}=t-t^{\prime}$ is bounded by $\pm 2 X^{0}$.

On the right of Fig. 6 we display the Wigner transform for the zero momentum mode as a function of $\omega$. One clearly observes that the interacting theory has a continuous spectrum described by a peaked spectral function with a nonzero width. The peak is located at $\omega_{0} / m_{R}=1.46$ in units of the initial renormalized mass (cf. Sec. 4.1.1), and the results are shown for $m_{R} X^{0}=35.1$ with $\lambda / m_{R}^{2}=4 .^{20}$ The inset shows a blow-up around $3 \omega_{0} / m_{R}=4.38$. The expected bump in the spectral function is small but visible. We stress that this bump in the spectral function is kinematically forbidden for the "onshell" approximation and arises from "off-shell" $1 \leftrightarrow 3$ processes. In Fig. 6 we also

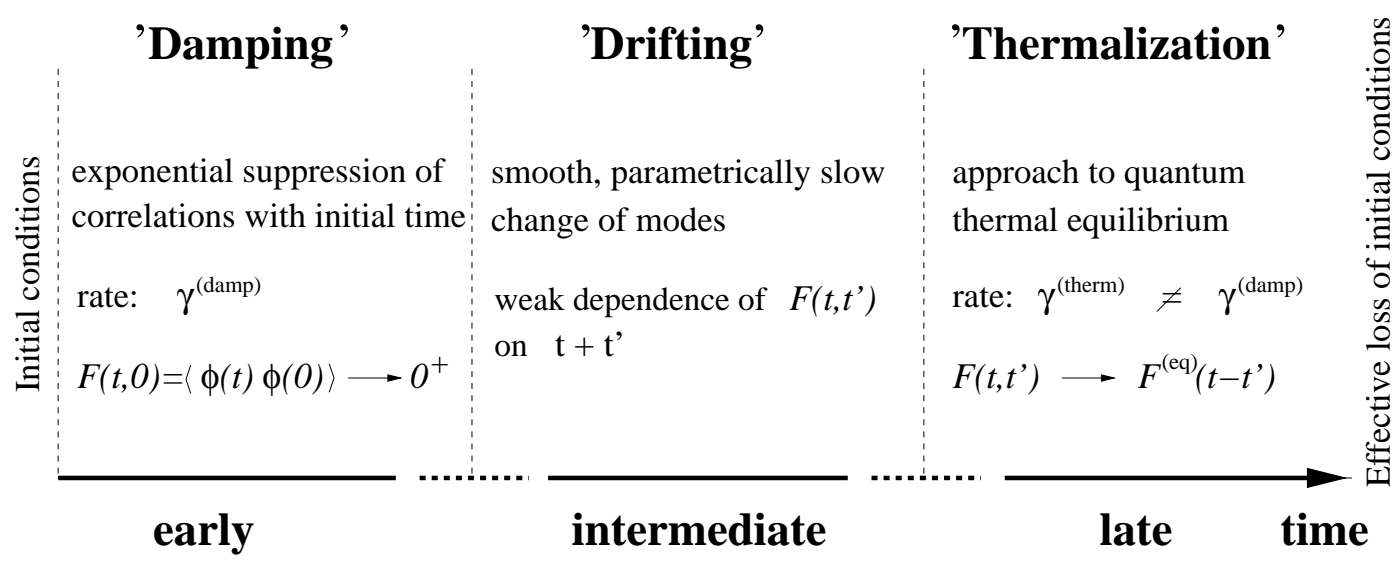

\footnotetext{
${ }^{20}$ Here we used a "tsunami" similar to the one discussed in Sec. 4.1.1, however, the shown results are insensitive to the initial condition details.
} 
present fits to a Breit-Wigner spectral function

$$
\rho_{\mathrm{BW}}\left(X^{0} ; \omega, \mathbf{p}\right)=\frac{2 \omega \Gamma_{\mathbf{p}}\left(X^{0}\right)}{\left[\omega^{2}-\omega_{\mathbf{p}}^{2}\left(X^{0}\right)\right]^{2}+\omega^{2} \Gamma_{\mathbf{p}}^{2}\left(X^{0}\right)},
$$

with a width $\Gamma_{\mathbf{p}}\left(X^{0}\right)=2 \gamma_{\mathbf{p}}^{(\mathrm{damp})}\left(X^{0}\right)$. While the position of the peak can be fitted easily, the overall shape and width are only qualitatively captured. In particular, the slope of $\rho\left(X^{0} ; \omega, p\right)$ for small $\omega$ is quantitatively different. We also see that the Breit-Wigner fits give a narrower spectral function (smaller width) and therefore would predict a slower exponential relaxation in real time.

The characteristic time scales observed for the nonequilibrium evolution of modes in Fig. 6 can be associated to

1. rapid oscillations of correlation functions with period $\sim 1 / \omega_{\mathbf{p}}$ described by the "peak" of the spectral function.

2. damping of oscillations with inverse rate $1 / \gamma_{\mathbf{p}}^{(\mathrm{damp})}$ described by a nonzero "width" $\Gamma_{\mathbf{p}}=2 \gamma_{\mathbf{p}}^{\text {(damp) }}$ of the spectral function. In equilibrium the "width" is given by

$$
2 \omega \Gamma^{(\mathrm{eq})}(\omega, \mathbf{p}) \equiv-\Sigma_{\rho}^{(\mathrm{eq})}(\omega, \mathbf{p}) \sim \mathscr{O}\left(\lambda^{2} / N\right) .
$$

3. late-time thermalization with inverse rate $1 / \gamma_{\mathbf{p}}^{\text {(therm) }}$ because of "off-shell" number changing processes. For the three-loop or NLO in $1 / N$ approximation of the $2 \mathrm{PI}$ effective action the processes changing the total particle number are perturbatively of order $\sim \lambda^{4} / N^{2}$ ("slow!"). This can be understood from the fact that the total particle number changing processes require a nonzero "width" $\sim \lambda^{2} / N$, and this width enters in $\mathscr{O}\left(\lambda^{2} / N\right)$ evolution equations for the two-point functions.

One finds qualitatively the same characteristic ranges for the corresponding nonequilibrium evolution in $3+1$ dimensions, and we will consider results for the chiral "quarkmeson" model below. An important quantitative difference between one and three spatial dimensions results from the fact that "on-shell" $2 \leftrightarrow 2$ processes contribute for the latter. However, as for the $1+1$ dimensional case they do not change the total "quasiparticle" number. Total number changing "off-shell" or "on-shell" processes are required in general to reach thermal equilibrium. As we have seen above, "off-shell" number changing processes appear at NLO in the $2 \mathrm{PI} 1 / N$ expansion. In contrast, they can be achieved "on-shell" first at NNLO. E.g. for $\phi^{4}$-theory the lowest order "on-shell" $(2 \rightarrow 4)$ contribution appears from the five-loop diagram (cf. Sec. 2.1):

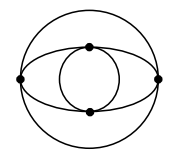

Perturbatively, this contribution is of the same order $\sim \lambda^{4} / N^{2}$ than the "off-shell" particle number changing processes arising at three-loop or NLO in the $1 / N$ expansion of the 2PI effective action. In this respect it is interesting to observe that precision tests for the nonequilibrium dynamics as discussed in Sec. 5 indicate accurate results already at NLO in the 2PI expansion. 


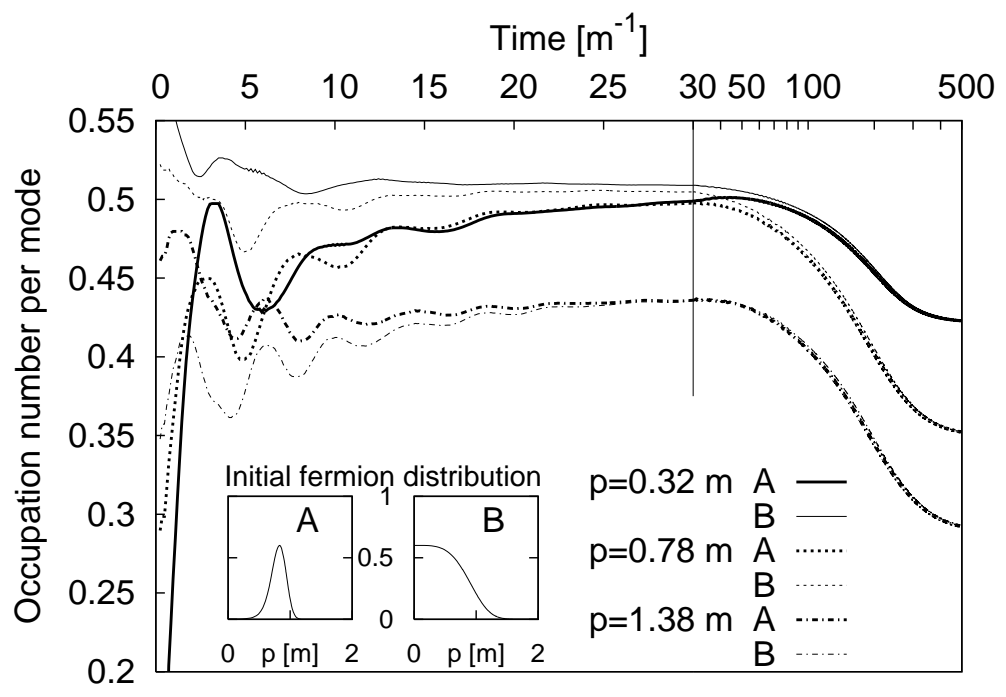

FIGURE 7. Fermion occupation number $n^{(f)}(t ; p)$ for three different momentum modes as a function of time. The evolution is shown for two different initial conditions with same energy density. The long-time behavior is shown on a logarithmic scale for $t \geq 30 \mathrm{~m}^{-1}$.

\subsection{Prethermalization}

Prethermalization is a universal far-from-equilibrium phenomenon which describes the very rapid establishment of an almost constant ratio of pressure over energy density (equation of state), as well as a kinetic temperature based on average kinetic energy. The phenomenon occurs on time scales dramatically shorter than the thermal equilibration time. As a consequence, prethermalized quantities approximately take on their final thermal values already at a time when the occupation numbers of individual momentum modes still show strong deviations from the late-time Bose-Einstein or Fermi-Dirac distribution.

Here we consider the nonequilibrium evolution of quantum fields for a low-energy quark-meson model, which is described in Sec. 2.3.1. It takes into account two quark flavors with a Yukawa coupling $\sim h$ to a scalar $\sigma$-field and a triplet of pseudoscalar pions, $\vec{\pi}$. The theory corresponds to the well-known "linear $\sigma$-model", which incorporates the chiral symmetries of massless two-flavor QCD. The employed couplings in the action (68) with (69) are taken to be of order one, and if not stated otherwise $h=\lambda=1$. We emphasize that the main results about prethermalization are independent of the detailed values of the couplings. Here we employ the 2PI effective action (70) to two-loop order given by (74). ${ }^{21}$ All quantities will be given in units of the scalar thermal mass $m .{ }^{22}$

Thermalization: In Fig. 7 we show the effective occupation number density of fermion

21 For the relation to a nonperturbative expansion of the 2PI effective action to next-to-leading order in $N_{f}$ see Secs. 2.3.1 and 2.4).

22 The thermal mass $m$ is evaluated in equilibrium. It is found to prethermalize very rapidly. The employed spatial momentum cutoff is $\Lambda / m=2.86$. 


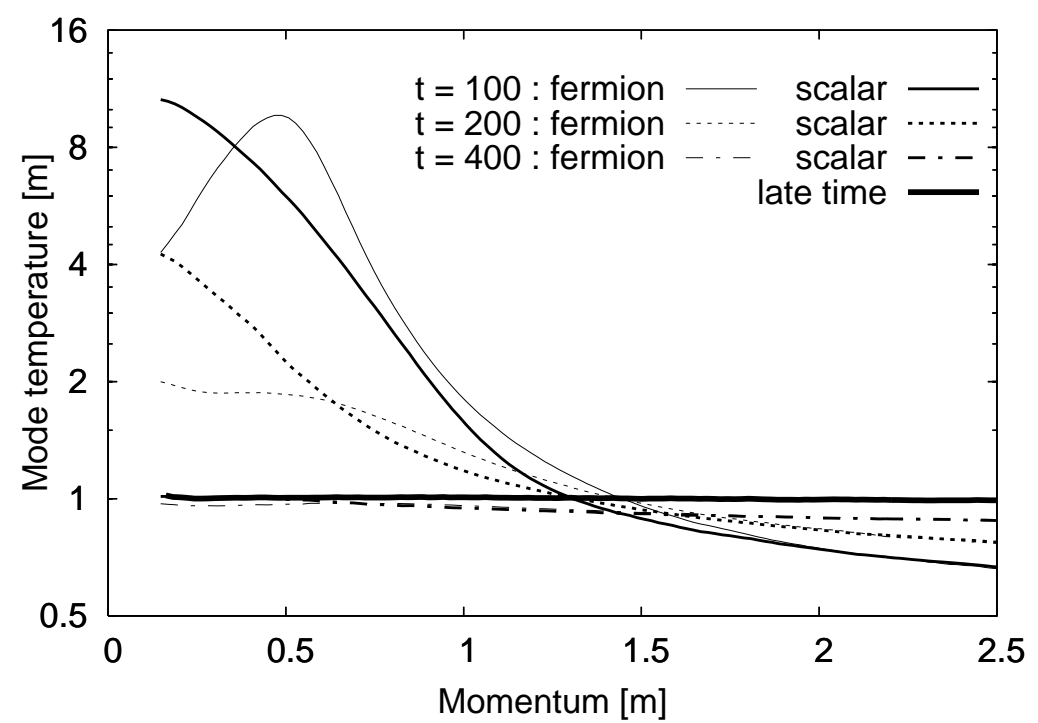

FIGURE 8. Fermion and scalar mode temperatures $T_{p}^{(f, s)}(t)$ as a function of momentum $p$ for various times.

momentum modes, $n^{(f)}(t ; p)$, as a function of time for three different momenta ${ }^{23}$. The plot shows two runs denoted as (A) and (B) with different initial conditions but same energy density. Run (A) exhibits a high initial particle number density in a narrow momentum range around $\pm p$. This situation is reminiscent of two colliding wave packets with opposite and equal momentum. We emphasize, however, that we are considering a spatially homogeneous and isotropic ensemble with a vanishing net charge density. For run (B) an initial particle number density is employed which is closer to a thermal distribution.

One observes that for a given momentum the mode numbers of run (A) and (B) approach each other at early times. The characteristic time scale for this approach is well described by the damping time $t_{\mathrm{damp}}(p)^{24}$. Irrespective of the initial distributions (A) or (B), we find (for $p / m \simeq 1$ ) $t_{\text {damp }}^{(f)} \simeq 25 m^{-1}$ for fermions and $t_{\text {damp }}^{(s)} \simeq 28 m^{-1}$ for scalars. In contrast to the initial rapid changes, one observes a rather slow or "quasistationary" subsequent evolution. The equilibration time $t_{\mathrm{eq}} \simeq 95 \mathrm{~m}^{-1}$ is substantially larger than $t_{\text {damp }}$ and is approximately the same for fermions and scalars. Thermal equilibration is a collective phenomenon which is, in particular, rather independent of the momentum. As we have also observed for the $1+1$ dimensional scalar theory in Sec. 4.1.4, mode quantities such as effective particle number distribution functions show a characteristic two-stage loss of initial conditions: after the damping time scale much of the details about the initial conditions are effectively lost. However, the system is still far from equilibrium and thermalization happens on a much larger time scale.

\footnotetext{
23 This quantity is directly related to the expectation value of the vector component of the field commutator $\langle[\psi, \bar{\psi}]\rangle$ in Wigner coordinates and fulfills $0 \leq n^{(\mathrm{f})}(t ; p) \leq 1$.

24 The rate $1 / t_{\text {damp }}(p)$ is determined by the spectral component of the self-energy.
} 


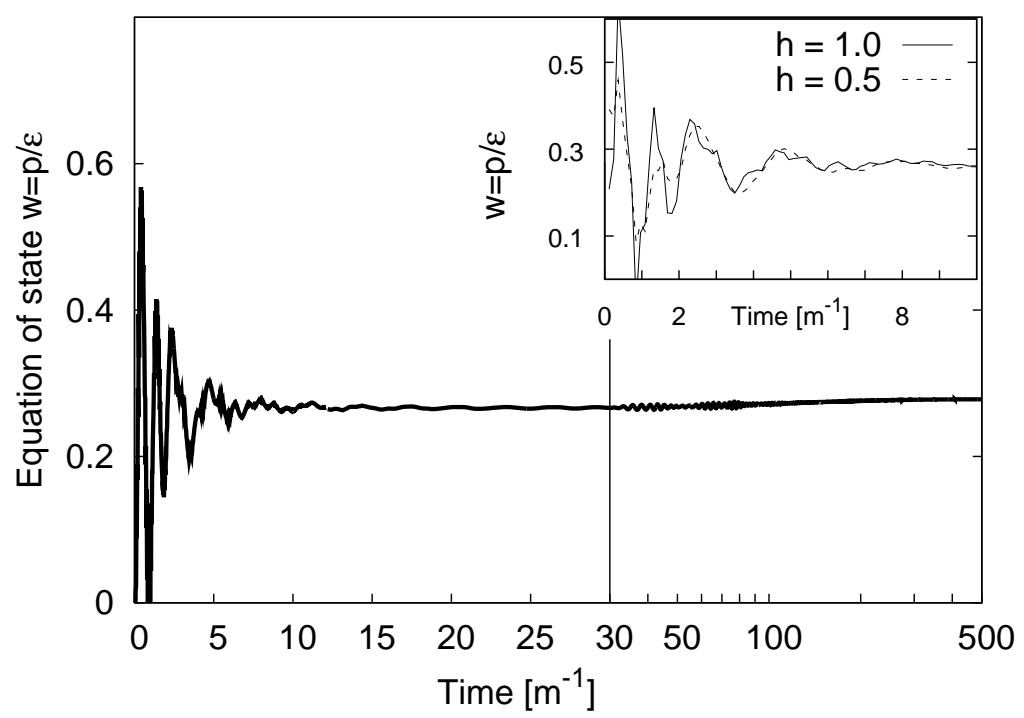

FIGURE 9. The ratio of pressure over energy density $w$ as a function of time. The inset shows the early stages for two different couplings and demonstrates that the prethermalization time is independent of the interaction details.

We define mode temperatures $T_{p}^{(f, s)}(t)$ by equating the mode particle numbers $n_{p}^{(f, s)}(t)$ with a time and momentum dependent Bose-Einstein or Fermi-Dirac distribution, respectively:

$$
n_{p}(t) \stackrel{!}{=}\left[\exp \left(\omega_{p}(t) / T_{p}(t)\right) \pm 1\right]^{-1} .
$$

This definition is a quantum mechanical version of its classical counterpart as defined by the squared "generalized velocities". In thermal equilibrium with $\omega_{p} \simeq \sqrt{p^{2}+M^{2}}$ and $T_{p}=T_{\text {eq }}$ equation (218) yields the familiar occupation numbers $(\mu=0)$. Here the mode frequency $\omega_{p}^{(f, s)}(t)$ is determined by the peak of the spectral function for given time and momentum, as detailed for the scalar theory in Sec. 4.1.4. In Fig. 8 we show the fermion and scalar mode temperature as a function of momentum for various times $t \gg t_{\mathrm{damp}}$. One observes that at late times, when thermal equilibrium is approached, all fermion and scalar mode temperatures become constant and agree: $T_{p}^{(f)}(t)=T_{p}^{(s)}(t)=T_{\text {eq }}$. In contrast, there are sizeable deviations from the thermal result even for times considerably larger than the characteristic damping time.

Kinetic prethermalization: In contrast to the rather long thermalization time, prethermalization sets in extremely rapidly. In Fig. 9 we show the ratio of pressure over energy density, $w=p / \varepsilon$, as a function of time. One observes that an almost time-independent equation of state builds up very early, even though the system is still far from equilibrium! The prethermalization time $t_{\mathrm{pt}}$ is here of the order of the characteristic inverse mass scale $m^{-1}$. This is a typical consequence of the loss of phase information by summing over oscillating functions with a sufficiently dense frequency spectrum. In order to see that this phenomenon is not related to scattering or to the strength of the interaction, we compare with a smaller coupling in the inset and observe good agreement of 


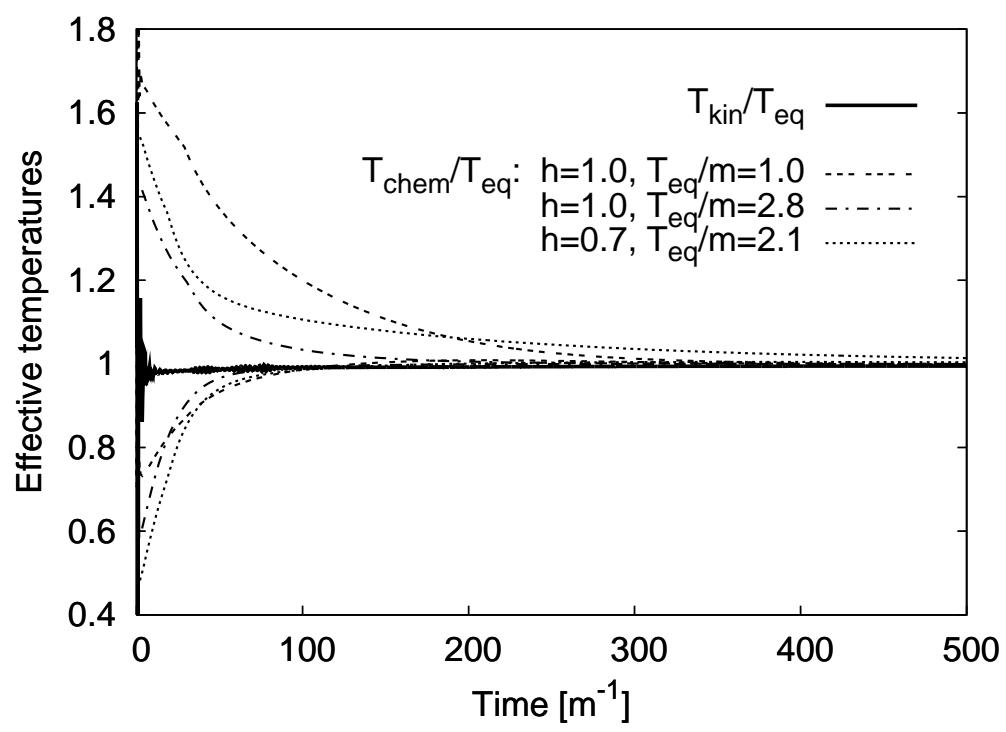

FIGURE 10. Chemical temperatures for scalars (upper curves) and fermions (lower curves) for different values of the coupling $h$ and $T_{\text {eq. }}$. We also show the kinetic temperature $T_{\text {kin }}(t)$ (solid line), which prethermalizes on a very short time scale as compared to chemical equilibration.

both curves. The dephasing phenomenon is unrelated to the scattering-driven process of thermalization.

Given an equation of state, the question arises whether there exists a suitable definition of a global kinetic temperature $T_{\text {kin. }}$. In contrast to a mode quantity such as $T_{p}(t)$, a temperature measure which averages over all momentum modes may prethermalize. Building on the classical association of temperature with the mean kinetic energy per degree of freedom, we use here a definition based on the total kinetic energy $E_{\text {kin }}(t)$ :

$$
T_{\text {kin }}(t)=E_{\text {kin }}(t) / c_{\text {eq }} .
$$

Here the extensive dimensionless proportionality constant $c_{\mathrm{eq}}=E_{\mathrm{kin}, \mathrm{eq}} / T_{\mathrm{eq}}$ is given solely in terms of equilibrium quantities ${ }^{25}$. Since total energy is conserved, the time scale when "equipartition" is reached (i.e. $E_{\text {kin }} / E$ is approximately constant) also corresponds to a time-independent kinetic temperature. The latter equals the equilibrium temperature $T_{\mathrm{eq}}$ if $E_{\mathrm{kin}} / E$ has reached the thermal value.

The solid line of Fig. 10 shows $T_{\text {kin }}(t)$ normalized to the equilibrium temperature (for $T_{\mathrm{eq}} / m=1$ ). One observes that an almost time-independent kinetic temperature is established after the short-time scale $t_{\mathrm{pt}} \sim m^{-1}$. The time evolution of bulk quantities such as the ratio of pressure over energy density $w$, or the kinetic temperature $T_{\text {kin }}$, are dominated by a single short-time scale. These quantities approximately converge to the thermal equilibrium values already at early times and can be used for an efficient "quasi-

25 For a relativistic plasma one has $E_{\mathrm{kin}} / N=\varepsilon / n=\alpha T$. As alternatives, one may consider the weighted average $\bar{T}(t)=\sum n(t ; p) T(t ; p) / \sum n(t ; p)$ where the sum is over all modes, or a definition analogous to Eq. (220) below. 
thermal" description in a far-from-equilibrium situation!

Chemical equilibration: In thermal equilibrium the relative particle numbers of different species are fixed in terms of temperature and particle masses. A system has chemically equilibrated if these ratios are reached, as observed for the hadron yields in heavy ion collisions. Obviously, the chemical equilibration time $t_{\mathrm{ch}}$ will depend on details of the particle number changing interactions in a given model and $t_{\mathrm{ch}} \leq t_{\mathrm{eq}}$. In our model we can study the ratio between the numbers of fermions and scalars. For this purpose we introduce the chemical temperatures $T_{\mathrm{ch}}^{(f, s)}(t)$ by equating the integrated number density of each species, $n^{(f, s)}(t)=g^{(f, s)} \int \mathrm{d}^{3} p /(2 \pi)^{3} n_{p}^{(f, s)}(t)$, with the integrated BoseEinstein/Fermi-Dirac form of distributions:

$$
n(t) \stackrel{!}{=} \frac{g}{2 \pi^{2}} \int_{0}^{\infty} \mathrm{d} p p^{2}\left[\exp \left(\omega_{p}(t) / T_{\mathrm{ch}}(t)\right) \pm 1\right]^{-1}
$$

Here $g^{(f)}=8$ counts the number of fermions and $g^{(s)}=4$ for the scalars.

The time evolution of the ratios $T_{\mathrm{ch}}^{(s, f)}(t) / T_{\mathrm{eq}}$ is shown in Fig. 10 for different values of the coupling constant $h$ and the equilibrium temperature $T_{\text {eq. }}$. One observes that chemical equilibration with $T_{\mathrm{ch}}^{(s)}(t)=T_{\mathrm{ch}}^{(f)}(t)$ does not happen on the prethermalization time scale, in contrast to the behavior of $T_{\text {kin }}(t)$. Being bulk quantities, the scalar and fermion chemical temperatures can approach each other rather quickly at first. Subsequently, a slow evolution towards equilibrium sets in. For the late-time chemical equilibration we find for our model $t_{\mathrm{ch}} \simeq t_{\mathrm{eq}}$. However, the deviation from the thermal result can become relatively small already for times $t \ll t_{\text {eq }}$.

Let us finally consider our findings in view of collisions of heavy nuclei and try to estimate the prethermalization time. Actually, $t_{\mathrm{pt}}$ is rather independent of the details of the model like particle content, values of couplings etc. It mainly reflects a characteristic frequency of the initial oscillations. If the "temperature" (i.e. average kinetic energy per mode) sets the relevant scale one expects $T t_{\mathrm{pt}}=$ const. (For low $T$ the scale will be replaced by the mass.) For our model we indeed find $T t_{\mathrm{pt}} \simeq 2-2.5$. ${ }^{26}$ We expect such a relation with a similar constant to hold for the quark-gluon state very soon after the collision. For $T \gtrsim 400-500 \mathrm{MeV}$ we obtain a very short prethermalization time $t_{\mathrm{pt}}$ of somewhat less than $1 \mathrm{fm}$. This is consistent with observed very early hydrodynamic behavior in collision experiments, however, one further has to investigate the required isotropization of pressure.

\subsection{Far-from-equilibrium field dynamics: Parametric resonance}

In classical mechanics parametric resonance is the phenomenon of resonant amplification of the amplitude of an oscillator having a time-dependent periodic frequency. In the context of quantum field theory a similar phenomenon describes the amplification of quantum fluctuations, which can be interpreted as particle production. It provides an

${ }^{26}$ We define $t_{\mathrm{pt}}$ by $\left|w\left(t_{\mathrm{pt}}\right)-w_{\mathrm{eq}}\right| / w_{\mathrm{eq}}<0.2$ for $t>t_{\mathrm{pt}}$. 
important building block for our understanding of the (pre)heating of the early universe at the end of an inflationary period, and may also be operative in various situations in the context of relativistic heavy-ion collision experiments. Here we will consider the phenomenon as a "paradigm" for far-from-equilibrium dynamics of macroscopic fields or one-point functions. Dynamics of correlation functions for vanishing - or similarly for constant - macroscopic fields has been described in detail above. There is a wealth of phenomena associated to non-constant fields such as parametric resonance, spinodal decomposition or in general the dynamics of phase transitions where the field can play the role of an order parameter.

The example of parametric resonance is particularly challenging since it is a nonperturbative phenomenon even in the presence of arbitrarily small couplings. Despite being a basic phenomenon that can occur in a large variety of quantum field theories, parametric resonance is a rather complex process, which in the past defied most attempts for a complete analytic treatment even for simple theories. It is a far-from-equilibrium phenomenon that involves densities inversely proportional to the coupling. The nonperturbatively large occupation numbers cannot be described by standard kinetic descriptions. So far, classical statistical field theory simulations on the lattice have been the only quantitative approach available. These are expected to be valid for not too late times, before the approach to quantum thermal equilibrium sets in (cf. also Sec. 5). Studies in quantum field theory have been mainly limited to linear or mean-field type approximations (leading-order in large- $N$, Hartree), which present a valid description for sufficiently early times. However, they are known to fail to describe thermalization and miss important rescattering effects (cf. also Sec. 4.1). The 2PI 1/ $N$ expansion provides for the first time a quantitative nonperturbative approach in quantum field theory taking into account rescattering.

Recall for a moment the classical mechanics example of resonant amplitude amplification for an oscillator with time-dependent periodic frequency. The amplitude $y(t)$ is described by the differential equation $\ddot{y}+\omega^{2}(t) y=0$ with periodic $\omega(t+T)=\omega(t)$ of period $T$. Since the equation is invariant under $t \rightarrow t+T$ there are periodic solutions $y(t+T)=c y(t)$. This can be expressed as $y(t)=c^{t / T} \Pi(t)$ with periodic $\Pi(t+T)=$ $\Pi(t)$. One concludes that for real $c>1$ there is an instability with an exponential growth. For small elongations a physical realization of this situation is a pendulum with a periodically changing length as displayed:

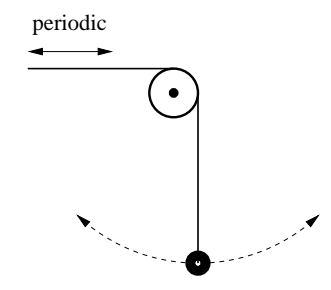

In contrast to the mechanics example, in quantum field theory there will be no external periodic source. A large coherent field amplitude coupled to its own quantum fluctuations will trigger the phenomenon of parametric resonance. Mathematically, however, important aspects are very similar to the above classical example for sufficiently early times: The mechanical oscillator amplitude $y$ plays the role of the statistical two-point function $F$ in quantum field theory, and the periodic $\omega^{2}(t)$ plays the role of an effec- 
tive mass term $M^{2}(\phi(t))$ whose time dependence is induced by an oscillating macroscopic field $\phi(t)$. Simple linear approximations to the problem, which have been much employed in the literature, are even mathematically equivalent to the above mechanics example. Accordingly, the well-known Lamé-type solutions of the mechanics problem will also play a role in the quantum field theory study. Substantial deviations do, however, quickly set in with important non-linear effects.

\subsubsection{Parametric resonance in the $O(N)$ model}

We consider the scalar $N$-component quantum field theory with classical action (13), and employ the 2PI 1/N-expansion to next-to-leading order. The relevant equations of motion are given by (183)-(189) as described in Sec. 3.6.1. We will describe numerical solutions of these equations in $3+1$ dimensions without further approximations. Moreover, the approach allows us to identify the relevant contributions to the dynamics at various times and to obtain an approximate analytic solution of the nonlinear dynamics for the entire amplification range. It should be emphasized that the approach solves the problem of an analytic description of the dynamics at nonperturbatively large densities. It is necessary to take into account the infinite set of NLO 2PI diagrams as described in Sec. 2.4.1. As we will show in the following, each of these eventually contributes to the same order in the coupling $\lambda$ such that any finite order in loops or couplings is not sufficient. In this sense, the 2 PI $1 / N$-expansion to NLO represents a minimal approach for the controlled description of the phenomenon. This justifies the rather involved complexity of the approximation.

We have in mind a situation reminiscent of that in the early universe after a period of chaotic inflation, driven by a macroscopic (inflaton) field. We consider a weakly coupled system that is initially in a pure quantum state, characterized by a large field amplitude

$$
\phi_{a}(t)=\sigma(t) M_{0} \sqrt{\frac{6 N}{\lambda}} \delta_{a 1} .
$$

and small quantum fluctuations, corresponding to vanishing particle numbers at initial time. Here $M_{0}$ sets our unit of mass, $\sigma(0)=\sigma_{0}$ is $\sim \mathscr{O}(1)$ and $\left.\partial_{t} \sigma(t)\right|_{t=0}=0$. The initial statistical propagator contains a "longitudinal" component $F_{\|}$and $(N-1)$ "transverse" components $F_{\perp}$ :

$$
F_{a b}=\operatorname{diag}\left\{F_{\|}, F_{\perp}, \ldots, F_{\perp}\right\},
$$

with $\left.\partial_{t} F(t, 0 ; \mathbf{p})\right|_{t=0}=0$, and $\left.\partial_{t} \partial_{t^{\prime}} F\left(t, t^{\prime} ; \mathbf{p}\right)\right|_{t=t^{\prime}=0} \equiv F^{-1}(0,0 ; \mathbf{p}) / 4$ for a pure-state initial density matrix (cf. Sec. 3.2).

To get an overview, in Fig. 11 we show the contributions from the macroscopic field and the from the fluctuations to the (conserved) total energy as functions of time for $N=4$ and $\lambda=10^{-6}{ }^{27}$ The total energy $E_{\text {tot }}$ is initially dominated by the classical part

27 Typical volumes $\left(N_{s} a_{s}\right)^{3}$ of $N_{s}=36-48$ with $a_{s}=0.4-0.3$ lead to results that are rather insensitive to finite-size and cutoff effects. 


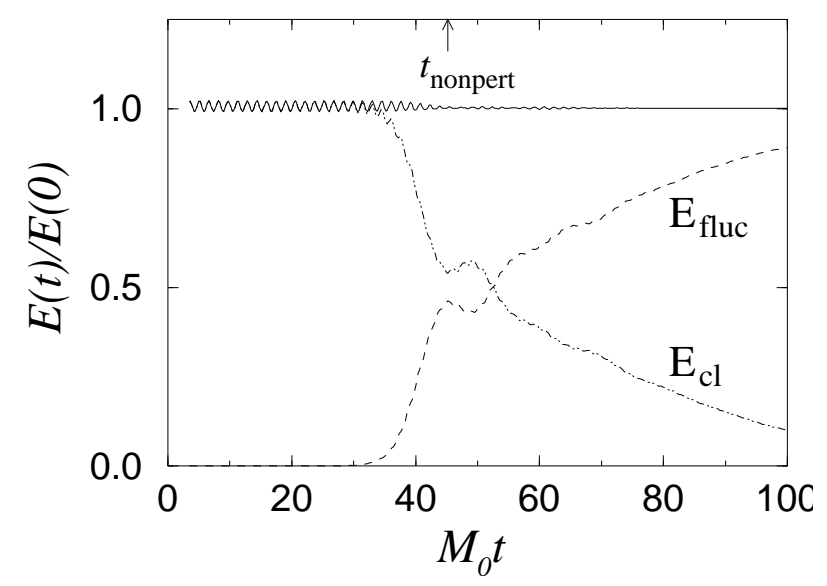

FIGURE 11. Total energy (solid line) and classical-field energy $E_{\mathrm{cl}}$ (dotted line) as a function of time. The dashed line represents the fluctuation part $E_{\text {fluc }}$, showing a transition from a classical-field to a fluctuation dominated regime.

$E_{\mathrm{cl}}(t=0)$, with reads in terms of the rescaled field (221):

$$
\frac{E_{\mathrm{cl}}(t)}{L_{3}}=\left.\frac{3 N}{\lambda} M_{0}^{2}\left[\partial_{t} \partial_{t^{\prime}}+m^{2}+\frac{1}{2} M_{0}^{2} \sigma^{2}(t)\right] \sigma(t) \sigma\left(t^{\prime}\right)\right|_{t=t^{\prime}}
$$

where $L_{3}$ denotes the spatial volume, and $E_{\mathrm{fluc}}=E_{\mathrm{tot}}-E_{\mathrm{cl}}$. More and more energy is converted into fluctuations as the system evolves. A characteristic time - denoted as $t_{\text {nonpert }}$ in Fig. 11 - is reached when both contributions become of the same size, i.e. $E_{\text {fluc }} \simeq E_{\mathrm{cl}}$. Before this time, the coherent oscillations of the field $\phi$ lead to a resonant enhancement of the statistical propagator Fourier modes

$$
F_{\perp}\left(t, t^{\prime} ; \mathbf{p}_{0}\right) \sim e^{\gamma_{0}\left(t+t^{\prime}\right)} .
$$

in a narrow range of momenta around a specific value $|\mathbf{p}| \simeq p_{0}$ : this is parametric resonance. Apart from the resonant amplification in the linear regime, we identify two characteristic time scales — denoted as $t_{\text {source }}$ and $t_{\text {collect }}$ below - , which signal strongly enhanced particle production in a broad momentum range. Nonlinear interactions between field modes cause the resonant amplification to spread to a broad range of momenta. More specifically, the initially amplified modes act as a source for other modes. The rate of the source-induced exponential amplification exceeds the characteristic rate $\gamma_{0}$ for the resonant amplification. This is illustrated in Figs. 12 and 13, where the effective particle numbers are shown for various momenta as a function of time in the transverse and the longitudinal sector. Before the transition to a fluctuation dominated regime around $t_{\text {nonpert }}$, one observes a rapid change of the particle numbers due to resonant as well as source-induced amplification. The source-induced amplification is crucial for the rapid approach to the subsequent, quasistationary regime, where direct scattering drive a very slow evolution towards thermal equilibrium. The transition to this slow regime around $t_{\text {nonpert }}$ can be very well observed from Figs. 12 and 13.

The fluctuation dominated regime is characterized by strong nonlinearities. For instance, from Fig. 11 one infers for $t \simeq t_{\text {nonpert }}$ that the classical field decay "overshoots" 


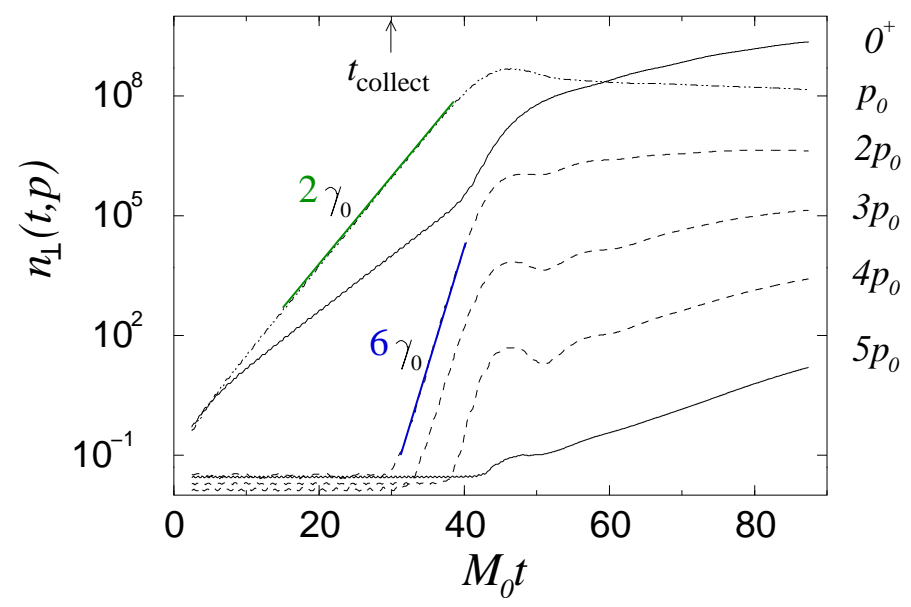

FIGURE 12. Effective particle number density for the transverse modes as a function of time for various momenta $p \leq 5 p_{0}$. At early times, modes with $p \simeq p_{0}$ are exponentially amplified with a rate $2 \gamma_{0}$. Due to nonlinearities, one observes subsequently an enhanced growth with rate $6 \gamma_{0}$ for a broad momentum range.

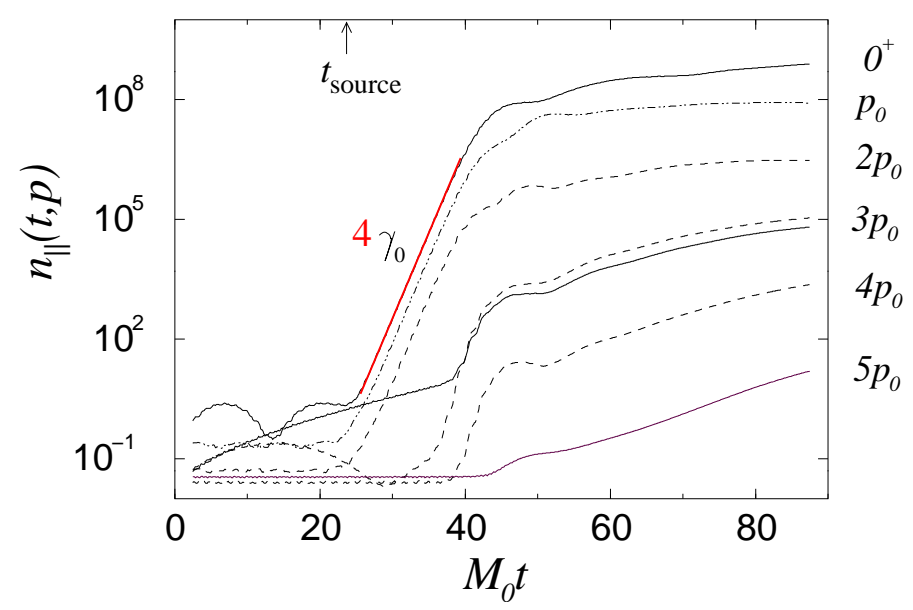

FIGURE 13. Same as in Fig. 12, for the longitudinal modes. Nonlinear source effects trigger an exponential growth with rate $4 \gamma_{0}$ for $p \lesssim 2 p_{0}$. The thick line corresponds to a mode in the parametric resonance band, and the long-dashed line for a similar one outside the band. The resonant amplification is quickly dominated by source-induced particle production.

and is temporarily reversed by feed-back from the modes. This can be directly seen in the evolution for the rescaled field shown in Fig. 14, which shows a dip around $t_{\text {nonpert }}$. (The particle numbers of Figs. 12 and 13 exhibit correspondingly a reverse behavior.) The oscillations in the envelope of $\sigma(t)$ damp out with time, and one observes a slow decay of the field and the associated energy at later times. The latter phenomena cannot be seen in leading-order or Hartree-type approximations and it is crucial to include the next-to-leading order contributions (cf. also Sec. 4.1.1). They are important for a reliable description of the system at the end of the resonance stage for finite $N \lesssim 1 / \lambda$. For realistic inflationary models with typically $\lambda \ll 1$ this is, in particular, crucial to determine whether there are any radiatively restored symmetries. 


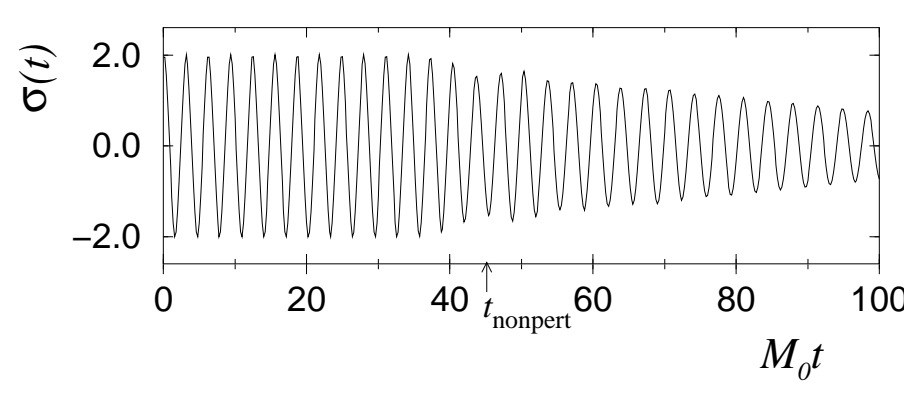

FIGURE 14. The rescaled field $\sigma$ as a function of time.

The characteristic properties described above can be understood analytically from the evolution equations for the one- and two-point functions. To set the scale we use the initial longitudinal mass squared $M_{0}^{2} \equiv M^{2}(t=0)$ with (cf. Eq. (146)):

$$
\begin{aligned}
M^{2}(t) & =m^{2}+\frac{\lambda}{6 N}\left[3 T_{\|}(t)+(N-1) T_{\perp}(t)\right], \\
T_{\|, \perp}(t) & =\int^{\Lambda} \frac{\mathrm{d}^{3} p}{(2 \pi)^{3}} F_{\|, \perp}(t, t ; \mathbf{p}),
\end{aligned}
$$

where we denote the "tadpole" contributions by $T_{\|}$and $T_{\perp}$ from the longitudinal and transverse propagator components, respectively, and some $\Lambda \gg p_{0}$. Initially, $F_{\|}(0,0 ; \mathbf{p})=1 /\left[2 \omega_{\|}(\mathbf{p})\right]$ with frequency $\omega_{\|}(\mathbf{p})=\left[\mathbf{p}^{2}+M_{0}^{2}\left(1+3 \sigma_{0}^{2}\right)\right]^{1 / 2}$, and similarly for the transverse components $F_{\perp}(0,0 ; \mathbf{p})$ where the frequency $\omega_{\perp}(\mathbf{p})$ contains a mass term $\sigma_{0}^{2}$ instead of $3 \sigma_{0}^{2}$. Parametrically the initial statistical propagator is of order one, i.e. $\sim \mathscr{O}\left(N^{0} \lambda^{0}\right)$. Parametric resonance leads to the dominant amplification of $F_{\perp}\left(t, t ; \mathbf{p}_{0}\right)$ with rate $2 \gamma_{0}$. As a consequence of the exponential amplification of the statistical propagator there is a characteristic time at which loop corrections will become of order one as well. This is schematically summarized in Fig. 15. The time when $F_{\perp}\left(t, t ; \mathbf{p}_{0}\right) \sim \mathscr{O}\left(N^{0} \lambda^{-1 / 2}\right)$ is denoted by $t=t_{\text {source. }}$. At this time the one-loop diagram with two field insertions indicated by crosses as depicted in Fig. 15 will give a contribution of order one to the evolution equation for $F_{\|}(t, t ; \mathbf{p})$. For instance, the two powers of the coupling coming from the vertices of that diagram are canceled by the field amplitudes (221) and by propagator lines associated to the amplified $F_{\perp}\left(t, t^{\prime} ; \mathbf{p}_{0}\right)$. Similarly, at the time $t=t_{\text {collect }}$ the maximally amplified transverse propagator mode has grown to $F_{\perp}\left(t, t^{\prime} ; \mathbf{p}_{0}\right) \sim \mathscr{O}\left(N^{1 / 3} \lambda^{-2 / 3}\right)$. As a consequence, the "setting sun" diagram in Fig. 15 becomes of order one and is therefore of the same order as the classical contributions. Though the loop corrections become of order one later than the initial time, they induce amplification rates that are multiples of the rate $\gamma_{0}$ which lead to a very rapid growth of modes in a wide momentum range. Finally, when the fluctuations have grown nonperturbatively large with $F_{\perp}\left(t, t^{\prime} ; \mathbf{p}_{0}\right) \sim \mathscr{O}\left(N^{0} \lambda^{-1}\right)$ any loop correction will no longer be suppressed by powers of the small coupling $\lambda$. In this case the nonperturbative $1 / N$ expansion becomes of crucial importance for a quantitative description of the dynamics to later times. In the following, we derive these results from the evolution equations (183)-(189) in more detail. 


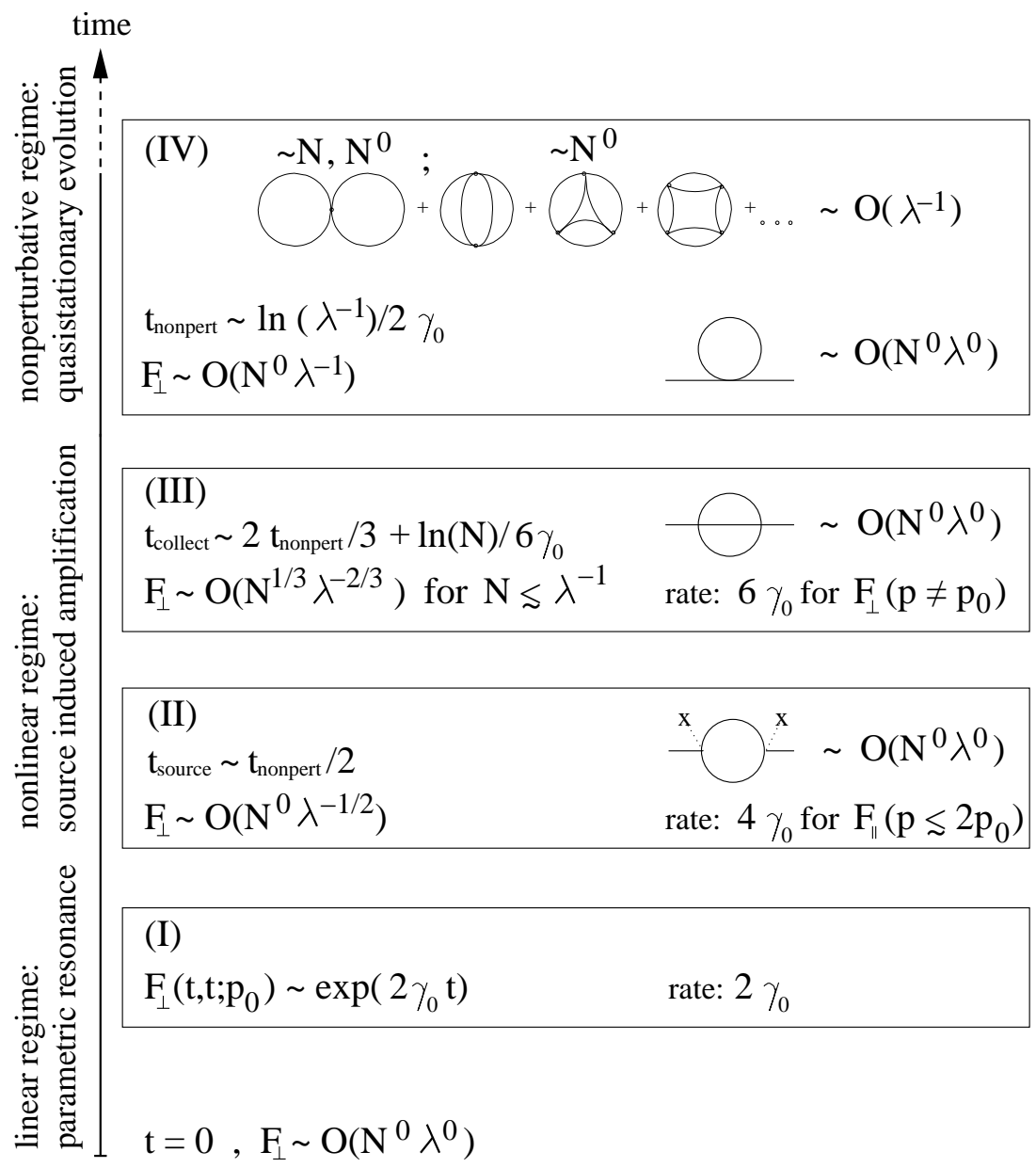

FIGURE 15. Schematic overview of the characteristic time scales and the respective relevant diagrammatic contributions (see text).

(I) Early-time (linear) regime: Resonant amplification. At early times the $\sigma$-field evolution equation receives the dominant, i.e. $\mathscr{O}\left(\lambda^{0}\right)$, contributions from the classical action $S$ given in (13). As a consequence, the field dynamics can be described by the classical equation of motion. In the following, all quantities are rescaled with appropriate powers of $M_{0}$ to become dimensionless and we set $M_{0} \equiv 1$. The classical field equation reads

$$
\partial_{t}^{2} \sigma(t)+\sigma(t)+\sigma^{3}(t)=0 .
$$

Differential equations of this time have been extensively studied in the literature. For the initial condition considered here it has the (anti-)periodic solution $\sigma\left(t+\pi / \omega_{0}\right)=-\sigma(t)$, which can be expressed in term of the Jacobian cosine cn as $\sigma(t)=\sigma_{0} \operatorname{cn}\left[t \sqrt{1+\sigma_{0}^{2}}, \sigma_{0} / \sqrt{2\left(1+\sigma_{0}^{2}\right)}\right]$. Therefore, the solution shows a rapidly oscillating behavior with characteristic frequency

$$
\omega_{0} \simeq 2 \sqrt{1+\sigma_{0}^{2}}
$$


The period average of the field amplitude can be also expressed in terms of the initial amplitude $\sigma_{0}$ as $\overline{\sigma^{2}(t)} \simeq \sigma_{0}^{2} / 2$.

The evolution equations for the two-point functions to order $\mathscr{O}\left(\lambda^{0}\right)$ correspond to free-field equations with the addition of a time-dependent mass term $\sim 3 \sigma^{2}(t)$ for the longitudinal and $\sim \sigma^{2}(t)$ for the transverse modes:

$$
\begin{aligned}
{\left[\partial_{t}^{2}+\mathbf{p}^{2}+1+\sigma^{2}(t)\right] F_{\perp}\left(t, t^{\prime} ; \mathbf{p}\right) } & =0 \\
{\left[\partial_{t}^{2}+\mathbf{p}^{2}+1+3 \sigma^{2}(t)\right] F_{\|}\left(t, t^{\prime} ; \mathbf{p}\right) } & =0,
\end{aligned}
$$

and equivalently for the spectral functions $\rho_{\perp}\left(t, t^{\prime} ; \mathbf{p}\right)$ and $\rho_{\|}\left(t, t^{\prime} ; \mathbf{p}\right)$. As a consequence of this approximation, the two-point functions can be factorized as products of singletime functions:

$$
\begin{aligned}
& F_{\perp}\left(t, t^{\prime} ; \mathbf{p}\right)=\frac{1}{2}\left[f_{\perp}(t ; \mathbf{p}) f_{\perp}^{*}\left(t^{\prime} ; \mathbf{p}\right)+f_{\perp}^{*}(t ; \mathbf{p}) f_{\perp}\left(t^{\prime} ; \mathbf{p}\right)\right], \\
& \rho_{\perp}\left(t, t^{\prime} ; \mathbf{p}\right)=i\left[f_{\perp}(t ; \mathbf{p}) f_{\perp}^{*}\left(t^{\prime} ; \mathbf{p}\right)-f_{\perp}^{*}(t ; \mathbf{p}) f_{\perp}\left(t^{\prime} ; \mathbf{p}\right)\right],
\end{aligned}
$$

and similarly for the longitudinal components. We emphasize that the simple decomposition (230) is no longer correct at higher orders in the coupling. In terms of these so-called mode functions the equations of motion read, e.g. for the transverse modes:

$$
\left[\partial_{t}^{2}+\mathbf{p}^{2}+1+\sigma^{2}(t)\right] f_{\perp}(t ; \mathbf{p})=0 .
$$

Up to an overall arbitrary phase, the above initial conditions for the two-point functions translate into $f_{\perp}(0, \mathbf{p})=1 / \sqrt{2 \omega_{\perp}(\mathbf{p})},\left.\partial_{t} f_{\perp}(t, \mathbf{p})\right|_{t=0}=-i \sqrt{\omega_{\perp}(\mathbf{p}) / 2}$ and equivalently for $f_{\|}$. For this approximation the quantum field theory problem becomes mathematically equivalent to the well-known classical mechanics problem described in the above introduction. The analytical solution of the Lamé-type equation (231) is well known and can be summarized for our purposes as follows: There is an exponential amplification of $F_{\perp}\left(t, t^{\prime} ; \mathbf{p}\right)$ for a bounded momentum range $0 \leq \mathbf{p}^{2} \leq \sigma_{0}^{2} / 2$. This corresponds to a maximum momentum for amplification $\mathbf{p}_{\max }^{2}+1+\overline{\sigma^{2}(t)} \simeq 1+\sigma_{0}^{2}=\left(\omega_{0} / 2\right)^{2}$. A further important result is that there is a separation of scales: $\omega_{0} \gg \gamma_{0}$, with the maximum amplification rate $\gamma_{0} \simeq 2 \delta \omega_{0}$ for $\mathbf{p}^{2}=\mathbf{p}_{0}^{2} \simeq \sigma_{0}^{2} / 4$ with the small number $\delta \leq e^{-\pi} \simeq 0.043$. There is much smaller growth in a narrow momentum range for $F_{\|}$, which is of no importance here since it is quickly dominated by loop corrections as is shown below. Time-averaged over $\sim \omega_{0}^{-1}$, for $t, t^{\prime} \gg \gamma_{0}^{-1}$ one finds the result of Eq. (224). Note here that also leading-order large- $N$ or Hartree-type approximations show the same bounded amplification regime, such that at all times no higher momentum modes would get populated. This is, of course, an artefact of the approximation which is absent once NLO corrections are taken into account as is described below.

The analytic results for the Lamé regime agree precisely with the NLO numerical results for sufficiently early times. Figs. 12 and 13 show the transverse and longitudinal particle numbers for various momenta in the range $0<p \leq 5 p_{0}$, averaged over the rapid oscillation time $\sim 1 / \omega_{0}$. For the transverse modes the lowest two momenta shown are 
inside the resonance band. We define the effective particle numbers as in Eq. (201). ${ }^{28} \mathrm{As}$ shown in Sec. 4.1.2 for the $O(N)$ model these definitions yield an efficient description approaching a Bose-Einstein distributed particle number at sufficiently late times.

(II) Source-induced (nonlinear) amplification regime: Strongly enhanced particle production for longitudinal modes. The $\mathscr{O}\left(\lambda^{0}\right)$ approximation for longitudinal modes breaks down at the time

$$
t \simeq t^{\prime}=t_{\text {source }}: F_{\perp}\left(t, t^{\prime} ; \mathbf{p}_{0}\right) \sim \mathscr{O}\left(N^{0} \lambda^{-1 / 2}\right) .
$$

This can be derived from the $\mathscr{O}(\lambda)$ evolution equations to which one-loop self-energies contribute, which diagrammatically are given by tion equation reads:

$$
\begin{aligned}
& \left(\partial_{t}^{2}+\mathbf{p}^{2}+M^{2}(t)+3 \sigma^{2}(t)\right) F_{\|}\left(t, t^{\prime} ; \mathbf{p}\right) \simeq \\
& \quad \frac{2 \lambda(N-1)}{3 N} \sigma(t)\left\{\int_{0}^{t} \mathrm{~d} t^{\prime \prime} \sigma\left(t^{\prime \prime}\right) \Pi_{\perp}^{\rho}\left(t, t^{\prime \prime} ; \mathbf{p}\right) F_{\|}\left(t^{\prime \prime}, t^{\prime} ; \mathbf{p}\right)\right. \\
& \left.\quad-\frac{1}{2} \int_{0}^{t^{\prime}} \mathrm{d} t^{\prime \prime} \sigma\left(t^{\prime \prime}\right) \Pi_{\perp}^{F}\left(t, t^{\prime \prime} ; \mathbf{p}\right) \rho_{\|}\left(t^{\prime \prime}, t^{\prime} ; \mathbf{p}\right)\right\} \equiv R H S
\end{aligned}
$$

Here we have abbreviated $\Pi_{\perp}^{A}\left(t, t^{\prime \prime} ; \mathbf{p}\right)=\int \mathrm{d}^{3} q /(2 \pi)^{3} F_{\perp}\left(t, t^{\prime \prime} ; \mathbf{p}-\mathbf{q}\right) A_{\perp}\left(t, t^{\prime \prime} ; \mathbf{q}\right)$ with $A=\{F, \rho\}$, and we used that $\Pi_{\perp} \gg \Pi_{\|}$and $F_{\perp}^{2} \gg \rho_{\perp}^{2} \cdot{ }^{29}$ One observes that indeed for $F_{\perp} \sim \mathscr{O}\left(N^{0} \lambda^{-1 / 2}\right)$ the r.h.s. of Eq. (233) becomes $\sim \mathscr{O}(1)$ and cannot be neglected.

In order to make analytical progress, one has to evaluate the "memory integrals" in the above equation. This is dramatically simplified by the fact that the integral is approximately local in time, since the exponential growth lets the latest-time contributions dominate the integral. (This will be the case for times $t \lesssim t_{\text {nonpert }}$ after which exponential amplification stops, cf. below.) For the approximate evaluation of the memory integrals we consider time-averages over $\omega_{0}^{-1} \ll \gamma_{0}^{-1}$ :

$$
\int_{0}^{t} \mathrm{~d} t^{\prime \prime} \longrightarrow \int_{t-c / \omega_{0}}^{t} \mathrm{~d} t^{\prime \prime} \quad(c \sim 1)
$$

and perform a Taylor expansion around the latest time $t\left(t^{\prime}\right)$ :

$$
\begin{aligned}
& \left.\rho_{\|, \perp}\left(t, t^{\prime \prime} ; \mathbf{p}\right) \simeq \partial_{t^{\prime \prime}} \rho_{\|, \perp}\left(t, t^{\prime \prime} ; \mathbf{p}\right)\right|_{t=t^{\prime \prime}}\left(t^{\prime \prime}-t\right) \equiv\left(t-t^{\prime \prime}\right) \\
& F_{\|, \perp}\left(t, t^{\prime \prime} ; \mathbf{p}\right) \simeq F_{\|, \perp}(t, t ; \mathbf{p})
\end{aligned}
$$

\footnotetext{
28 We have employed $Q \equiv 0$ as in Sec. 4.1.2 which is, however, irrelevant.

29 The latter inequality will be discussed in detail in Sec. 5, where it is shown to indicate the validity of classical statistical field theory approximations.
} 
where we have used the equal-time commutation relations (117). With these approximations the r.h.s. of Eq. (233) can be evaluated as:

$$
\begin{aligned}
R H S & \simeq \lambda \sigma^{2}(t) \frac{c^{2}}{\omega_{0}^{2}} \frac{(N-1)}{3 N} T_{\perp}(t) F_{\|}\left(t, t^{\prime} ; \mathbf{p}\right) & \quad(\text { mass term }) \\
& +\lambda \sigma(t) \sigma\left(t^{\prime}\right) \frac{c^{2}}{\omega_{0}^{2}} \frac{(N-1)}{6 N} \Pi_{\perp}^{F}\left(t, t^{\prime} ; \mathbf{p}\right) & \quad \text { (source term) }
\end{aligned}
$$

The first term is a contribution from NLO to the mass, whereas the second term represents a source. Note that both the LO mass term and the above correction to this mass are of the same order in $\lambda$, however, with opposite sign. To evaluate the momentum integrals, we use a saddle point approximation around the dominant $p \simeq p_{0}$, valid for $t, t^{\prime} \gg \gamma_{0}^{-1}$, with $F_{\perp}\left(t, t^{\prime}, \mathbf{p}\right) \simeq F_{\perp}\left(t, t^{\prime}, \mathbf{p}_{0}\right) \exp \left[-\left|\gamma_{0}^{\prime}\right|\left(t+t^{\prime}\right)\left(p-p_{0}\right)^{2} / 2\right] .{ }^{30}$ From this one obtains for the above mass term:

$$
\lambda T_{\perp}(t) \simeq \lambda \frac{p_{0}^{2} F_{\perp}\left(t, t ; \mathbf{p}_{0}\right)}{2\left(\pi^{3}\left|\gamma_{0}^{\prime \prime}\right| t\right)^{1 / 2}} .
$$

The result can be used to obtain an estimate at what time $t$ this loop correction becomes an important contribution to the evolution equation. Note that to this order in $\lambda$ it is correct to use $F_{\perp}\left(t, t^{\prime} ; \mathbf{p}_{0}\right) \sim e^{\gamma_{0}\left(t+t^{\prime}\right)}$ on the r.h.s. of (238). The condition $\lambda T_{\perp}\left(t=t_{\text {nonpert }}\right)$ $\sim \mathscr{O}(1)$ can then be written for $\lambda \ll 1$ as:

$$
t_{\text {nonpert }} \simeq \frac{1}{2 \gamma_{0}} \ln \lambda^{-1}
$$

The same saddle point approximation can be performed to evaluate the source term (237):

$$
\lambda \Pi_{\perp}^{F}\left(t, t^{\prime} ; 0\right) \simeq \lambda \frac{p_{0}^{2} F_{\perp}^{2}\left(t, t^{\prime} ; \mathbf{p}_{0}\right)}{4\left(\pi^{3}\left|\gamma_{0}^{\prime \prime}\right|\left(t+t^{\prime}\right)\right)^{1 / 2}} .
$$

Here we only wrote the source term for $\mathbf{p}=0$ where it has its maximum, although it affects all modes with $p \lesssim 2 p_{0}$. Again this can be used to estimate the time $t=t_{\text {source }}$ at which $\lambda \Pi_{\perp}^{F} \sim \mathscr{O}(1)$ :

$$
t_{\text {source }} \simeq \frac{1}{2} t_{\text {nonpert }}
$$

One arrives at the important conclusion that the source term (237) becomes earlier of order one than the mass term (236): For $t_{\text {source }} \lesssim t \lesssim t_{\text {nonpert }}$ the source term dominates the dynamics! Using these estimates in (233) one finds that the longitudinal modes with $0 \lesssim p \lesssim 2 p_{0}$ get amplified with twice the rate $2 \gamma_{0}$ :

$$
F_{\|}(t, t ; \mathbf{p}) \sim \lambda F_{\perp}^{2}\left(t, t ; \mathbf{p}_{0}\right) \sim \lambda e^{4 \gamma_{0} t}
$$

${ }^{30} \operatorname{Here} \gamma(p) \simeq \gamma_{0}+\gamma_{0}^{\prime \prime}\left(p-p_{0}\right)^{2} / 2$ with $\gamma_{0}^{\prime \prime} \simeq-64 \delta(1-6 \delta) \sqrt{1+\sigma_{0}^{2}} / \sigma_{0}^{2}$. 
Though the non-linear contributions start later, they grow twice as fast! The analytical estimates for $t_{\text {source }}$ and rates agree very well with the numerical results shown in Fig. 13.

(III) Collective amplification regime: explosive particle production in a broad momentum range for transverse modes. A similar analysis can be made for the transverse modes. Beyond the Lamé-type $\mathscr{O}\left(\lambda^{0}\right)$ description, the evolution equation for $F_{\perp}$ receives contributions from the feed-back of the longitudinal modes at $\mathscr{O}(\lambda)$ as well as from the amplified transverse modes at $\mathscr{O}\left(\lambda^{2}\right)$. They represent source terms in the evolution equation for $F_{\perp}\left(t, t^{\prime} ; \mathbf{p}\right)$ which are both parametrically of the form $\sim \lambda^{2} F_{\perp}^{3} / N$ as is depicted below:

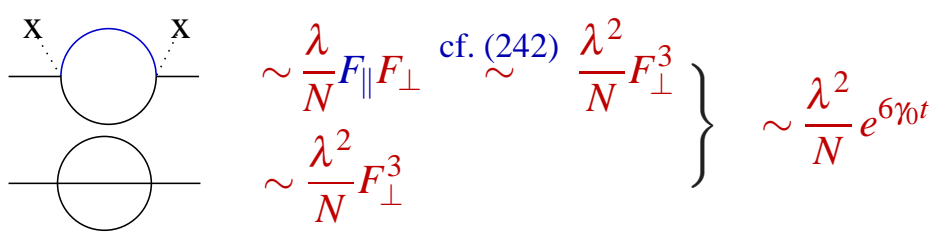

Following along the lines of the above paragraph and using (239) this leads to the characteristic time $t=t_{\text {collect }}$ at which these source terms become of order one:

$$
t_{\text {collect }} \simeq \frac{2}{3} t_{\text {nonpert }}+\frac{\ln N}{6 \gamma_{0}}
$$

For $t \simeq t^{\prime} \simeq t_{\text {collect }}$ the dominant transverse mode has grown to

$$
F_{\perp}\left(t, t^{\prime} ; \mathbf{p}_{0}\right) \sim \mathscr{O}\left(N^{1 / 3} \lambda^{-2 / 3}\right) .
$$

Correspondingly, for $t_{\text {collect }} \lesssim t \lesssim t_{\text {nonpert }}$ one finds a large particle production rate $\sim 6 \gamma_{0}$ in a momentum range $0 \lesssim p \lesssim 3 p_{0}$, in agreement with the full NLO results shown in Fig. 12. In this time range the longitudinal modes exhibit an enhanced amplification as well (cf. Fig. 13). It is important to realize that the phenomenon of source-induced amplification repeats itself: the newly amplified modes, together with the primarily amplified ones, act as a source for other modes, and so on. In this way, even higher growth rates of multiples of $\gamma_{0}$ can be observed and the "explosive" amplification rapidly propagates towards higher momentum modes. We emphasize that the collective amplification regime is absent in the LO large- $N$ approximation. Consequently, even for the transverse sector the latter does not give an accurate description if $t_{\text {collect }} \leq t_{\text {nonpert }}$, that is for $N \lesssim \lambda^{-1}$.

Behavior of the field. Around $t \lesssim t_{\text {nonpert }}$ is the earliest time when sizeable corrections to the classical field equation (227) appear, which give (cf. Eq. 189):

$$
\left[\partial_{t}^{2}+1+\delta M^{2}(t)+\sigma^{2}(t)\right] \sigma(t)=-\left.\int_{0}^{t} \mathrm{~d} t^{\prime} \Sigma_{\|}^{\rho}\left(t, t^{\prime} ; \mathbf{p}=0\right)\right|_{\sigma=0} \sigma\left(t^{\prime}\right)
$$

where $\delta M^{2} \simeq \frac{\lambda(N-1)}{6 N} T_{\perp}$. Before $t_{\text {nonpert }}$, where the "memory expansion" discussed above is valid, we can discuss the behavior of the field $\sigma=\sigma^{(0)}+\delta \sigma$ perturbatively in terms of 
a slowly varying small correction $\delta \sigma$ to the classical solution $\sigma^{(0)}$ of (227) and a small $\delta M^{2}$. If we neglect for a moment the NLO contributions on the r.h.s. the linearized equation (245) then yields

$$
\sigma(t) \simeq\left(1-\frac{1}{1+3 \sigma_{0}^{2}} \frac{\lambda(N-1)}{6 N} T_{\perp}(t)\right) \sigma^{(0)}(t) .
$$

One concludes that there is an exponential decrease of the field amplitude at LO since $T_{\perp}(t) \sim e^{2 \gamma_{0} t}$ for $t \lesssim t_{\text {nonpert }}$ according to (238). The dominant NLO corrections read

$$
\begin{array}{r}
\left.\left.\int_{t-c / \omega_{0}}^{t} d t^{\prime} \Sigma_{\|}^{\rho}\left(t, t^{\prime} ; \mathbf{p}=0\right)\right|_{\sigma=0} \sigma\left(t^{\prime}\right) \simeq \frac{c^{2}}{2 \omega_{0}^{2}}\left(\left.\partial_{t} \Sigma_{\|}^{\rho}\left(t, t^{\prime} ; \mathbf{p}=0\right)\right|_{\sigma=0}\right)\right|_{t=t^{\prime}} \sigma(t) \\
\stackrel{\mathscr{O}\left(\lambda^{2}\right)}{\simeq}-\frac{c^{2}}{2 \omega_{0}^{2}} \frac{\lambda^{2}}{18 N} \int^{\Lambda} \frac{\mathrm{d}^{3} q}{(2 \pi)^{3}} \frac{\mathrm{d}^{3} k}{(2 \pi)^{3}} F_{\perp}(t, t ;-\mathbf{q}-\mathbf{k}) F_{\perp}(t, t ; \mathbf{k}) \sigma(t) \\
\simeq-\frac{c^{2}}{2 \omega_{0}^{2}} \frac{\lambda^{2}}{18 N} T_{\perp}^{2}(t) \sigma(t)
\end{array}
$$

Note that the NLO correction to the effective mass term comes with an opposite sign than the LO correction. For $t \rightarrow t_{\text {nonpert }}$ one has $\delta M^{2} \sim \mathscr{O}\left(N^{0} \lambda^{0}\right)$ and $\Sigma_{\|}^{\rho} \sim \mathscr{O}\left(N^{-1} \lambda^{0}\right)$, i.e. they become of the same order in $\lambda$. As a consequence, cancellations may lead (temporarily) to reverse field decay. This is indeed what one observes from the full numerical NLO solution for $N=4$ and $\lambda=10^{-6}$ shown in Fig. 14. Around $t_{\text {nonpert }}$ strong nonlinearities appear, where the field decay "overshoots" and is shortly reversed by feedback from modes, overshoots again etc.

(IV) Fluctuation dominated regime: nonperturbatively large densities $\sim 1 / \lambda$ and quasistationary evolution. When the system evolves in time, more and more energy is converted into fluctuations. The description in terms of $\mathscr{O}\left(\lambda^{2}\right)$ evolution equations break down at

$$
t \simeq t^{\prime}=t_{\text {nonpert }}: \quad F_{\perp}\left(t, t^{\prime} ; \mathbf{p}_{0}\right) \sim \mathscr{O}\left(N^{0} \lambda^{-1}\right) .
$$

Transverse and longitudinal modes have grown to $\mathscr{O}\left(N^{0} \lambda^{-1}\right)$ in a wide momentum range for times $t \gtrsim t_{\text {nonpert }}$. This corresponds to nonperturbatively large particle number densities $n_{\perp}(\mathbf{p})$ and $n_{\|}(\mathbf{p})$ inversely proportional to the coupling. Because of this parametric dependence there are leading contributions to the dynamics coming from all loop orders. As a consequence, shortly after $t_{\text {nonpert }}$ a comparably slow, quasistationary evolution sets. It is important to note that descriptions based on a finite-order loop expansion of the 2PI effective action cannot be applied. To describe this regime it is crucial to employ a nonperturbative approximation as provided by the $2 \mathrm{PI} 1 / N$ expansion at NLO.

In order to discuss this in more detail, we consider the self-energies appearing in the evolution equations (138) for $F_{\perp}\left(t, t^{\prime} ; \mathbf{p}\right)$ and $\rho_{\perp}\left(t, t^{\prime} ; \mathbf{p}\right)$. From the NLO expressions given in (184) and (185) one finds for $\Sigma_{\perp}^{F}$ :

$$
\Sigma_{\perp}^{F}\left(t, t^{\prime} ; \mathbf{p}\right)=-\frac{\lambda}{3 N} \int \frac{\mathrm{d}^{3} q}{(2 \pi)^{3}}\{
$$




$$
\begin{gathered}
I_{F}\left(t, t^{\prime} ; \mathbf{q}\right) F_{\perp}\left(t, t^{\prime} ; \mathbf{p}-\mathbf{q}\right)-\frac{1}{4} I_{\rho}\left(t, t^{\prime} ; \mathbf{q}\right) \rho_{\perp}\left(t, t^{\prime} ; \mathbf{p}-\mathbf{q}\right) \\
\left.+\quad P_{F}\left(t, t^{\prime} ; \mathbf{q}\right) F_{\perp}\left(t, t^{\prime} ; \mathbf{p}-\mathbf{q}\right)-\frac{1}{4} P_{\rho}\left(t, t^{\prime} ; \mathbf{q}\right) \rho_{\perp}\left(t, t^{\prime} ; \mathbf{p}-\mathbf{q}\right)\right\} .
\end{gathered}
$$

The functions $I_{F, \rho}$ and $P_{F, \rho}$ contain the summation of an infinite number of "chain" graphs, where each additional element adds another loop to the graph (cf. also the figures in Sec. 2.4.1). We will argue in the following that each loop order of this infinite number of graphs contributes with the same order in the coupling $\lambda$.

According to (181) and (182) the sum of "chain" graphs described by $I_{F, \rho}$ can be iteratively generated by the relations

$$
\begin{array}{r}
I_{F}\left(t, t^{\prime} ; \mathbf{q}\right)=-\frac{\lambda}{3 N} \Pi_{F}\left(t, t^{\prime} ; \mathbf{q}\right)+\frac{\lambda}{3 N} \int_{0}^{t} d t^{\prime \prime} I_{\rho}\left(t, t^{\prime \prime} ; \mathbf{q}\right) \Pi_{F}\left(t^{\prime \prime}, t^{\prime} ; \mathbf{q}\right) \\
-\frac{\lambda}{3 N} \int_{0}^{t^{\prime}} d t^{\prime \prime} I_{F}\left(t, t^{\prime \prime} ; \mathbf{q}\right) \Pi_{\rho}\left(t^{\prime \prime}, t^{\prime} ; \mathbf{q}\right), \\
I_{\rho}\left(t, t^{\prime} ; \mathbf{q}\right)=-\frac{\lambda}{3 N} \Pi_{\rho}\left(t, t^{\prime} ; \mathbf{q}\right)+\frac{\lambda}{3 N} \int_{t^{\prime}}^{t} d t^{\prime \prime} I_{\rho}\left(t, t^{\prime \prime} ; \mathbf{q}\right) \Pi_{\rho}\left(t^{\prime \prime}, t^{\prime} ; \mathbf{q}\right),
\end{array}
$$

and corresponding expressions for $P_{F, \rho}$ (cf. Eqs. (186) and (187)). Here the "chain elements" $\Pi_{F, \rho}$ are given by

$$
\begin{aligned}
\Pi_{F}\left(t, t^{\prime} ; \mathbf{q}\right)= & -\frac{1}{2} \int \frac{\mathrm{d}^{3} k}{(2 \pi)^{3}}\left\{F_{\|}\left(t, t^{\prime} ; \mathbf{q}-\mathbf{k}\right) F_{\|}\left(t, t^{\prime} ; \mathbf{k}\right)\right. \\
& +(N-1) F_{\perp}\left(t, t^{\prime} ; \mathbf{q}-\mathbf{k}\right) F_{\perp}\left(t, t^{\prime} ; \mathbf{k}\right)-\frac{1}{4}\left[\rho_{\|}\left(t, t^{\prime} ; \mathbf{q}-\mathbf{k}\right)\right. \\
& \left.\left.\rho_{\|}\left(t, t^{\prime} ; \mathbf{k}\right)+(N-1) \rho_{\perp}\left(t, t^{\prime \prime} ; \mathbf{q}-\mathbf{k}\right) \rho_{\perp}\left(t, t^{\prime \prime} ; \mathbf{k}\right)\right]\right\} \\
\Pi_{\rho}\left(t, t^{\prime} ; \mathbf{q}\right)= & -\int \frac{\mathrm{d}^{3} k}{(2 \pi)^{3}}\left\{F_{\|}\left(t, t^{\prime} ; \mathbf{q}-\mathbf{k}\right) \rho_{\|}\left(t, t^{\prime} ; \mathbf{k}\right)\right. \\
& \left.+(N-1) F_{\perp}\left(t, t^{\prime} ; \mathbf{q}-\mathbf{k}\right) \rho_{\perp}\left(t, t^{\prime} ; \mathbf{k}\right)\right\}
\end{aligned}
$$

Denoting a given loop order $l$ by $I_{F}^{(l)}$ we can write

$$
\begin{aligned}
I_{F}\left(t, t^{\prime} ; \mathbf{q}\right)= & \sum_{l=1}^{\infty} I_{F}^{(l)}\left(t, t^{\prime} ; \mathbf{q}\right) \\
I_{F}^{(1)}\left(t, t^{\prime} ; \mathbf{q}\right)= & -\frac{\lambda}{3 N} \Pi_{F}\left(t, t^{\prime} ; \mathbf{q}\right) \\
I_{F}^{(2)}\left(t, t^{\prime} ; \mathbf{q}\right)= & -\left(\frac{\lambda}{3 N}\right)^{2} \int_{0}^{t} d t^{\prime \prime} \Pi_{\rho}\left(t, t^{\prime \prime} ; \mathbf{q}\right) \Pi_{F}\left(t^{\prime \prime}, t^{\prime} ; \mathbf{q}\right) \\
& +\left(\frac{\lambda}{3 N}\right)^{2} \int_{0}^{t^{\prime}} d t^{\prime \prime} \Pi_{F}\left(t, t^{\prime \prime} ; \mathbf{q}\right) \Pi_{\rho}\left(t^{\prime \prime}, t^{\prime} ; \mathbf{q}\right)
\end{aligned}
$$


and similarly for $I_{\rho}=\sum_{l=1}^{\infty} I_{\rho}^{(l)}$. Concentrating on the transverse modes, one observes from (252) and (253) that

$$
\Pi_{F}\left(t, t^{\prime} ; 0\right) \sim \mathscr{O}\left(N \lambda^{-2}\right), \quad \Pi_{\rho}\left(t, t^{\prime} ; 0\right) \sim \mathscr{O}\left(N \lambda^{-1}\right) .
$$

Inserting this into (250) or (254) for $I_{F}$, and into the corresponding expression (251) for $I_{\rho}$, one finds

$$
I_{F}^{(l)}\left(t, t^{\prime} ; 0\right) \sim \mathscr{O}\left(N^{0} \lambda^{-1}\right), \quad I_{\rho}^{(l)}\left(t, t^{\prime} ; 0\right) \sim \mathscr{O}\left(N^{0} \lambda^{0}\right),
$$

irrespective of the loop order $l$. Here it is important to note that averaged over the rapid oscillation time the spectral functions are of order one:

$$
\rho_{\perp}\left(t, t^{\prime} ; \mathbf{p}\right) \sim \rho_{\|}\left(t, t^{\prime} ; \mathbf{p}\right) \sim \mathscr{O}\left(N^{0} \lambda^{0}\right)
$$

As a consequence, with (257) the self-energy (250) receives leading contributions in the coupling from all loop orders. Since these contributions are proportional $N^{-1} \lambda^{0}$, they are subleading in $1 / N$. In particular, the expansion in powers of $1 / N$ employed here remains a valid nonperturbative approximation scheme. It is interesting to note that the 2PI $1 / N$ expansion to NLO can be understood as a "three-loop" approximation with an effective vertex. This is denoted schematically below for the graphs appearing at NLO:

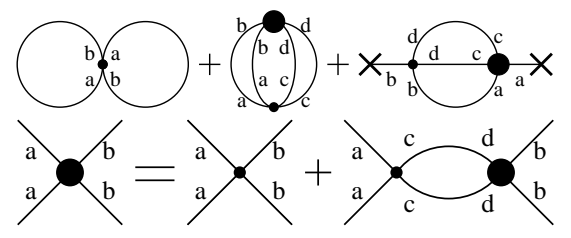

The effect of the nonperturbatively large densities is taken into account by a selfconsistent vertex correction as indicated by the diagrammatic equation for the fourvertex. The question of how self-consistent vertex corrections can be systematically described for cases where no $1 / N$ expansion is applicable will be treated in Sec. 6. We finally note that for times $t \gtrsim t_{\text {nonpert }}$ the evolution equations are no longer characterized by the effective time-locality in the sense described above such that the "memory expansion" cannot be used for an approximate description at late times.

\section{CLASSICAL ASPECTS OF NONEQUILIBRIUM QUANTUM FIELDS: PRECISION TESTS}

It is an important question to what extend nonequilibrium quantum field theory can be approximated by classical statistical field theory. It is a frequently employed strategy in the literature to consider nonequilibrium classical dynamics instead of quantum dynamics since the former can be simulated numerically up to controlled statistical errors. Classical statistical field theory indeed gives important insights when the number of field quanta per mode is sufficiently large such that quantum fluctuations are suppressed compared to statistical fluctuations. We will derive below a sufficient condition for the validity of classical approximations to nonequilibrium dynamics. The description in terms 
of spectral and statistical correlation functions as introduced in Sec. 3.4.1 is particularly suitable for comparisons since these correlation functions possess a well-defined classical limit. However, classical Rayleigh-Jeans divergences and the lack of genuine quantum effects - such as the approach to quantum thermal equilibrium characterized by Bose-Einstein or Fermi-Dirac statistics - limit the use of classical statistical field theory. To find out its use and its limitations we perform below direct comparisons of nonequilibrium classical and quantum evolution for same initial conditions. One finds that classical methods can give an accurate description of quantum dynamics for the case of large enough initial occupation numbers and not too late times, before the approach to quantum thermal equilibrium sets in. Classical approaches are unsuitable, in particular, to determine thermalization rates.

Classical methods have been extensively used in the past to rule out "candidates" for approximation schemes applied to nonequilibrium quantum field theory. Approximations that fail to describe classical nonequilibrium dynamics should be in general discarded also for the quantum case. If the dynamics is formulated in terms of correlation functions then approximation schemes for the quantum evolution can be straightforwardly implemented as well for the respective classical theory. For instance, the 2PI $1 / N$-expansion introduced in Sec. 2.4 can be equally well implemented in the classical as in the quantum theory. Therefore, in the classical statistical approach one can compare NLO results with results including all orders in $1 / N$. This gives a rigorous answer to the question of what happens at NNLO or beyond in this case. In particular, for increasing occupation numbers per mode the classical and the quantum evolution can be shown to approach each other if the same initial conditions are applied and for not too late times. For sufficiently high particle number densities one can therefore strictly verify how rapidly the $1 / N$ series converges for far-from-equilibrium dynamics! The possibility of precision tests are an important aspect of classical statistical field theory methods.

\subsection{Exact classical time-evolution equations}

In this section we define the basic classical correlation functions and derive exact dynamical equations for them. The evolution equations for the classical spectral function and the classical statistical propagator will turn out to be of the same form as Eqs. (138) for the corresponding quantum correlators - with the only difference that the quantum self-energies are replaced by the respective classical ones. The comparison between quantum and classical evolution can be very clearly discussed using the the language of correlation functions, since the quantum spectral function $\rho(x, y)$ and the statistical propagator $F(x, y)$ of Eq. (116) both have a well-defined classical equivalent.

We consider the classical $N$-component scalar field $\varphi_{a}$ with action (13). The classical field equation of motion is then given by

$$
\left[\square_{x}+m^{2}+\frac{\lambda}{6 N} \varphi_{b}(x) \varphi_{b}(x)\right] \varphi_{a}(x)=0,
$$

whose solution requires specification of the initial conditions $\varphi_{a}(0, \mathbf{x})=\varphi_{a}(\mathbf{x})$ and 
$\pi_{a}(0, \mathbf{x})=\pi_{a}(\mathbf{x})$ with $\pi_{a}(x) \equiv \partial_{x^{0}} \varphi_{a}(x)$. We define the macroscopic or average classical field:

$$
\phi_{a, \mathrm{cl}}(x)=\left\langle\varphi_{a}(x)\right\rangle_{\mathrm{cl}} \equiv \int D \pi D \varphi W[\pi, \varphi] \varphi_{a}(x) .
$$

Here $W[\pi, \varphi]$ denotes the normalized probability functional at initial time. The measure indicates integration over classical phase-space:

$$
\int D \pi D \varphi=\int \prod_{a=1}^{N} \prod_{\mathbf{x}} \mathrm{d} \pi_{a}(\mathbf{x}) \mathrm{d} \varphi_{a}(\mathbf{x}) .
$$

Here the theory will be defined on a spatial lattice to regulate the Rayleigh-Jeans divergence of classical statistical field theory. The connected classical statistical propagator $F_{a b, \mathrm{cl}}(x, y)$ is defined by

$$
F_{a b, \mathrm{cl}}(x, y)+\phi_{a, \mathrm{cl}}(x) \phi_{b, \mathrm{cl}}(y)=\left\langle\varphi_{a}(x) \varphi_{b}(y)\right\rangle_{\mathrm{cl}} \equiv \int D \pi D \varphi W[\pi, \varphi] \varphi_{a}(x) \varphi_{b}(y) .
$$

The classical equivalent of the quantum spectral function is obtained by replacing $-i$ times the commutator with the Poisson bracket: ${ }^{31}$

$$
\rho_{a b, \mathrm{cl}}(x, y)=-\left\langle\left\{\varphi_{a}(x), \varphi_{b}(y)\right\}_{\text {PoissonBracket }}\right\rangle_{\mathrm{cl}} .
$$

As a consequence, one finds the equal-time relations for the classical spectral function:

$$
\left.\rho_{a b, \mathrm{cl}}(x, y)\right|_{x^{0}=y^{0}}=0,\left.\quad \partial_{x^{0}} \rho_{a b, \mathrm{cl}}(x, y)\right|_{x^{0}=y^{0}}=\delta_{a b} \boldsymbol{\delta}(\mathbf{x}-\mathbf{y}) .
$$

Though their origin is different, note that they are in complete correspondence with the respective quantum relations (117).

The evolution equations for the classical statistical correlators can be obtained by functional methods in a similar way as for the quantum theory. In order not to be too redundant, we employ here a different approach starting from the differential equation for the free correlators. We will choose here $W[\pi, \varphi]$ to be invariant under the $O(N)$ symmetry with $\phi_{a} \equiv 0$ such that

$$
F_{a b, \mathrm{cl}}(x, y)=F_{\mathrm{cl}}(x, y) \delta_{a b},
$$

and equivalently for $\rho_{a b, \mathrm{cl}}(x, y) .{ }^{32}$ We will discuss $\phi_{a} \neq 0$ below. The unperturbed spectral function is a solution of the homogeneous equation ${ }^{33}$

$$
\left[\square_{x}+m^{2}\right] \rho_{0}(x, y)=0,
$$

31 Recall that the Poisson bracket with respect to the initial fields is

$$
\{A(x), B(y)\}_{\text {PoissonBracket }}=\sum_{a=1}^{N} \int \mathrm{d}^{d} z\left[\frac{\delta A(x)}{\delta \varphi_{a}(\mathbf{z})} \frac{\delta B(y)}{\delta \pi_{a}(\mathbf{z})}-\frac{\delta A(x)}{\delta \pi_{a}(\mathbf{z})} \frac{\delta B(y)}{\delta \varphi_{a}(\mathbf{z})}\right] .
$$

32 An explicit example for $W[\pi, \varphi]$ would be $W[\pi, \varphi]=Z_{0}^{-1} \exp \left\{\int \mathrm{d}^{d} \mathbf{x}\left(\pi^{2}+(\nabla \varphi)^{2}+m^{2} \varphi^{2}\right) / 2\right\}$ where $Z_{0} \equiv \int D \pi D \varphi W[\pi, \varphi]$. We emphasize that none of the following derivations will make use of a specific form for $W[\pi, \varphi]$.

33 This is the case both in the quantum and the classical theory. 
with initial conditions determined by the equal-time canonical relations (264). Let $F_{0}$ denote the solution of the unperturbed homogeneous problem

$$
\left[\square_{x}+m^{2}\right] F_{0}(x, y)=0
$$

with initial conditions determined by the initial probability functional. The derivation of the time evolution equations of classical correlation functions is conveniently formulated by introducing two additional two-point functions: the classical retarded and advanced Green functions, which are related to the classical spectral function $\rho_{\mathrm{cl}}$ by

$$
G_{\mathrm{cl}}^{R}(x, y)=\Theta\left(x^{0}-y^{0}\right) \rho_{\mathrm{cl}}(x, y)=G_{\mathrm{cl}}^{A}(y, x) .
$$

The classical retarded self-energy $\Sigma_{R, \mathrm{cl}}(x, y)$ can be defined as the difference between the full and free inverse retarded Green functions

$$
\Sigma_{R, \mathrm{cl}}(x, y)=\left(G_{\mathrm{cl}}^{R}\right)^{-1}(x, y)-\left(G_{0}^{R}\right)^{-1}(x, y),
$$

where the free retarded Green function

$$
G_{0}^{R}(x, y)=\Theta\left(x^{0}-y^{0}\right) \rho_{0}(x, y),
$$

solves the inhomogeneous equation

$$
\left[\square_{x}+m^{2}\right] G_{0}^{R}(x, y)=\delta^{d+1}(x-y),
$$

with retarded boundary conditions. In the same way we define the advanced self-energy $\Sigma_{A, \mathrm{cl}}$. Retarded (advanced) Green functions and self-energies vanish when $x^{0}<y^{0}$ $\left(x^{0}>y^{0}\right)$. With these definitions we can rewrite (269) and the respective equation for the advanced Green function as:

$$
\begin{aligned}
& G_{\mathrm{cl}}^{R}=G_{0}^{R}-G_{0}^{R} \cdot \Sigma_{R, \mathrm{cl}} \cdot G_{\mathrm{cl}}^{R}, \\
& G_{\mathrm{cl}}^{A}=G_{0}^{A}-G_{0}^{A} \cdot \Sigma_{A, \mathrm{cl}} \cdot G_{\mathrm{cl}}^{A},
\end{aligned}
$$

where we use a compact notation

$$
A \cdot B=\int \mathrm{d}^{d+1} z A(x, z) B(z, y) .
$$

A combination of Eqs. (272) and (273) gives the Schwinger-Dyson equation for the classical spectral function $\rho_{\mathrm{cl}}=G_{\mathrm{cl}}^{R}-G_{\mathrm{cl}}^{A}$ with

$$
\rho_{\mathrm{cl}}=\rho_{0}-G_{0}^{R} \cdot \Sigma_{R, \mathrm{cl}} \cdot G_{\mathrm{cl}}^{R}+G_{0}^{A} \cdot \Sigma_{A, \mathrm{cl}} \cdot G_{c l}^{A} \cdot
$$

There is a similar identity for the classical statistical propagator $F_{\mathrm{cl}}$, defined in (261). Using the definitions (272) and (273) one can write the following identity for the statistical function

$$
F_{\mathrm{cl}}=F_{0}-G_{0}^{R} \cdot\left[\Sigma_{R, \mathrm{cl}}-G_{\mathrm{cl}}^{R^{-1}}\right] \cdot F_{\mathrm{cl}}-F_{0} \cdot\left[\Sigma_{A, \mathrm{cl}}+G_{0}^{A^{-1}}\right] \cdot G_{\mathrm{cl}}^{A} .
$$


We can now define a classical statistical self-energy as

$$
\Sigma_{F, \mathrm{cl}}=-G_{\mathrm{cl}}^{R^{-1}} \cdot F_{\mathrm{cl}} \cdot G_{\mathrm{cl}}^{A-1}+G_{0}^{R^{-1}} \cdot F_{0} \cdot G_{0}^{A^{-1}},
$$

and find the Schwinger-Dyson equation for the statistical propagator:

$$
F_{\mathrm{cl}}=F_{0}-G_{0}^{R} \cdot \Sigma_{R, \mathrm{cl}} \cdot F_{\mathrm{cl}}-F_{0} \cdot \Sigma_{A, \mathrm{cl}} \cdot G_{\mathrm{cl}}^{A}-G_{0}^{R} \cdot \Sigma_{F, \mathrm{cl}} \cdot G_{\mathrm{cl}}^{A} \cdot
$$

Acting with $\left(\square+m^{2}\right)$ on (275) and (278) brings the classical Schwinger-Dyson equations in a form which is more suitable for initial-value problems. We make the retarded nature of $\Sigma_{R, \mathrm{cl}}$ manifest by writing

$$
\Sigma_{R, \mathrm{cl}}(x, y)=\Sigma_{\mathrm{cl}}^{(0)}(x) \delta^{d+1}(x-y)+\Theta\left(x^{0}-y^{0}\right) \Sigma_{\rho, \mathrm{cl}}(x, y),
$$

and similarly for $\Sigma_{A, \mathrm{cl}}(x, y)$. The spectral component of the classical self-energy is $\Sigma_{\rho, \mathrm{cl}}(x, y)=\Sigma_{R, \mathrm{cl}}(x, y)-\Sigma_{A, \mathrm{cl}}(x, y)=-\Sigma_{\rho, \mathrm{cl}}(y, x)$. After properly taking into account all $\Theta$-functions as well as (266), (271) and (267), one finds for the exact time evolution equations for $\rho_{\mathrm{cl}}$ and $F_{\mathrm{cl}}$ :

$$
\begin{aligned}
{\left[\square_{x}+M_{\mathrm{cl}}^{2}(x)\right] \rho_{\mathrm{cl}}(x, y)=} & -\int_{y^{0}}^{x^{0}} \mathrm{~d} z \Sigma_{\rho, \mathrm{cl}}(x, z) \rho_{\mathrm{cl}}(z, y), \\
{\left[\square_{x}+M_{\mathrm{cl}}^{2}(x)\right] F_{\mathrm{cl}}(x, y)=} & -\int_{0}^{x^{0}} \mathrm{~d} z \Sigma_{\rho, \mathrm{cl}}(x, z) F_{\mathrm{cl}}(z, y) \\
& +\int_{0}^{y^{0}} \mathrm{~d} z \Sigma_{F, \mathrm{cl}}(x, z) \rho_{\mathrm{cl}}(z, y),
\end{aligned}
$$

where we use the abbreviated notation $\int_{t_{1}}^{t_{2}} \mathrm{~d} z \equiv \int_{t_{1}}^{t_{2}} \mathrm{~d} z^{0} \int_{-\infty}^{\infty} \mathrm{d}^{d} z$ and

$$
M_{\mathrm{cl}}^{2}(x)=m^{2}+\Sigma_{\mathrm{cl}}^{(0)}(x) \text {. }
$$

We emphasize that the form of these classical time evolution equations is the same as for the quantum evolution described by (138). If the initial conditions are chosen to be the same then the only difference between classical and quantum theory originates from the self-energies entering the evolution equations. As a direct consequence, one concludes that LO large- $N$ or any Gaussian/Hartree type approximation exhibits purely classical dynamics: since the quantum self-energies $\Sigma_{F}$ and $\Sigma_{\rho}$ as well as the classical $\Sigma_{F, \text { cl }}$ and $\Sigma_{\rho, \mathrm{cl}}$ vanish for these approximations the evolution equations derived from the quantum theory are identical to those derived from the classical theory. The tadpole contributions $\Sigma^{(0)}$ and $\Sigma_{\mathrm{cl}}^{(0)}$ entail no difference if the initial conditions are chosen to be the same. In the following we consider approximations with non-vanishing spectral and statistical selfenergies in order to discuss the differences between classical and quantum dynamics.

\subsection{Classicality condition}

The classical self-energies $\Sigma_{\rho, \mathrm{cl}}, \Sigma_{F, \mathrm{cl}}(x, z)$ and $\Sigma_{\mathrm{cl}}^{(0)}$ could be approximated by perturbation theory to a given order in the coupling $\lambda$. However, as for the case of the quantum 
evolution the perturbative classical self-energies lead to a secular time-evolution and fail to provide a reliable description for the classical nonequilibrium dynamics. The analytical description can, however, be based on the same 2PI summation techniques as introduced for the quantum theory above. We will only state here the result of the respective calculation within classical statistical field theory, since we will then discuss how the same result can be directly obtained from the classical limit of the known quantum self-energies.

One finds for the classical statistical $O(N)$ model employing the $2 \mathrm{PI} 1 / N$ expansion to next-to-leading order:

$$
\begin{aligned}
M_{\mathrm{cl}}^{2}(x) & =m^{2}+\lambda \frac{N+2}{6 N} F_{\mathrm{cl}}(x, x), \\
\Sigma_{F, \mathrm{cl}}^{(\mathrm{NLO})}(x, y) & =-\frac{\lambda}{3 N} F_{\mathrm{cl}}(x, y) I_{F, \mathrm{cl}}(x, y), \\
\Sigma_{\rho, \mathrm{cl}}^{(\mathrm{NLO})}(x, y) & =-\frac{\lambda}{3 N}\left(F_{\mathrm{cl}}(x, y) I_{\rho, \mathrm{cl}}(x, y)+\rho_{\mathrm{cl}}(x, y) I_{F, \mathrm{cl}}(x, y)\right),
\end{aligned}
$$

with the classical summation functions

$$
\begin{aligned}
I_{F, \mathrm{cl}}(x, y) & =\frac{\lambda}{6} F_{\mathrm{cl}}^{2}(x, y) \\
& -\frac{\lambda}{6} \int \mathrm{d}^{d} z\left\{\int_{0}^{x^{0}} d z^{0} I_{\rho, \mathrm{cl}}(x, z) F_{\mathrm{cl}}^{2}(z, y)-2 \int_{0}^{y^{0}} d z^{0} I_{F, \mathrm{cl}}(x, z) F_{\mathrm{cl}}(z, y) \rho_{\mathrm{cl}}(z, y)\right\} \\
I_{\rho, \mathrm{cl}}(x, y) & =\frac{\lambda}{3} F_{\mathrm{cl}}(x, y) \rho_{\mathrm{cl}}(x, y)-\frac{\lambda}{3} \int \mathrm{d}^{d} z \int_{y^{0}}^{x^{0}} d z^{0} I_{\rho, \mathrm{cl}}(x, z) F_{\mathrm{cl}}(z, y) \rho_{\mathrm{cl}}(z, y) .
\end{aligned}
$$

At this stage we can compare the result from the $2 \mathrm{PI} 1 / N$ expansion for the quantum theory, as discussed in Sec. 2.4, with the resummed classical expressions obtained here. One observes that the classical self-energies (283) - (284) are obtained from the corresponding quantum expressions (178)—(182) by dropping the terms with a product of two spectral functions $\rho$ compared to a product of two statistical propagators $F$. Accordingly, the classical self-energies are obtained from the respective quantum ones as

$$
\Sigma_{F, \mathrm{cl}}=\Sigma_{F}\left(F^{2} \gg \rho^{2}\right) \quad, \quad \Sigma_{\rho, \mathrm{cl}}=\Sigma_{\rho}\left(F^{2} \gg \rho^{2}\right) .
$$

The same analysis can be done employing the 2PI loop expansion. For the following analytical discussion we consider the spatially homogeneous case and employ the Fourier modes $\Sigma_{F, \text { cl }}\left(t, t^{\prime} ; \mathbf{p}\right)$ and $\Sigma_{\rho, \text { cl }}\left(t, t^{\prime} ; \mathbf{p}\right)$. For the classical statistical $O(N)$ symmetric theory one obtains to two-loop order the self-energies:

$$
\begin{aligned}
& \Sigma_{F, \mathrm{cl}}^{(2 \text { loop })}\left(t, t^{\prime} ; \mathbf{p}\right)=-\lambda^{2} \frac{N+2}{18 N^{2}} \int_{\mathbf{q}, \mathbf{k}} F_{\mathrm{cl}}\left(t, t^{\prime} ; \mathbf{p}-\mathbf{q}-\mathbf{k}\right) F_{\mathrm{cl}}\left(t, t^{\prime} ; \mathbf{q}\right) F_{\mathrm{cl}}\left(t, t^{\prime} ; \mathbf{k}\right), \\
& \Sigma_{\rho, \mathrm{cl}}^{(2 \text { loop })}\left(t, t^{\prime} ; \mathbf{p}\right)=-\lambda^{2} \frac{N+2}{6 N^{2}} \int_{\mathbf{q}, \mathbf{k}} \rho_{\mathrm{cl}}\left(t, t^{\prime} ; \mathbf{p}-\mathbf{q}-\mathbf{k}\right) F_{\mathrm{cl}}\left(t, t^{\prime} ; \mathbf{q}\right) F_{\mathrm{cl}}\left(t, t^{\prime} ; \mathbf{k}\right) .
\end{aligned}
$$


Let us directly confront this with the respective two-loop self-energies for the respective quantum $O(N)$ theory:

$$
\begin{aligned}
\Sigma_{F}^{(2 \text { loop })}\left(t, t^{\prime} ; \mathbf{p}\right)= & -\lambda^{2} \frac{N+2}{18 N^{2}} \int_{\mathbf{q}, \mathbf{k}} F\left(t, t^{\prime} ; \mathbf{p}-\mathbf{q}-\mathbf{k}\right) \\
& {\left[F\left(t, t^{\prime} ; \mathbf{q}\right) F\left(t, t^{\prime} ; \mathbf{k}\right)-\frac{3}{4} \rho\left(t, t^{\prime} ; \mathbf{q}\right) \rho\left(t, t^{\prime} ; \mathbf{k}\right)\right] } \\
\Sigma_{\rho}^{(2 \text { loop })}\left(t, t^{\prime} ; \mathbf{p}\right)= & -\lambda^{2} \frac{N+2}{6 N^{2}} \int_{\mathbf{q}, \mathbf{k}} \rho\left(t, t^{\prime} ; \mathbf{p}-\mathbf{q}-\mathbf{k}\right) \\
& {\left[F\left(t, t^{\prime} ; \mathbf{q}\right) F\left(t, t^{\prime} ; \mathbf{k}\right)-\frac{1}{12} \rho\left(t, t^{\prime} ; \mathbf{q}\right) \rho\left(t, t^{\prime} ; \mathbf{k}\right)\right] . }
\end{aligned}
$$

From this one infers a sufficient condition for classical evolution:

$$
\left|F\left(t, t^{\prime} ; \mathbf{q}\right) F\left(t, t^{\prime} ; \mathbf{k}\right)\right| \gg \frac{3}{4}\left|\rho\left(t, t^{\prime} ; \mathbf{q}\right) \rho\left(t, t^{\prime} ; \mathbf{k}\right)\right|
$$

This condition has to be fulfilled for all times and all momenta in order to ensure that classical and quantum evolution agree. However, we will observe below that it can be typically only achieved for a limited range of time and/or momenta.

One expects that the classical description becomes a reliable approximation for the quantum theory if the number of field quanta in each mode is sufficiently high. The classicality condition (288) entails the justification of this expectation. In order to illustrate the condition in terms of a more intuitive picture of occupation numbers, we employ the free-field theory type form of the spectral function and statistical propagator given in Eq. (211). From this one obtains the following estimates for the time-averaged correlators:

$$
\begin{aligned}
& \overline{F^{2}}\left(t, t^{\prime} ; \mathbf{p}\right) \equiv \frac{\omega_{\mathbf{p}}}{2 \pi} \int_{t-2 \pi / \omega_{\mathbf{p}}}^{t} \mathrm{~d} t^{\prime} F^{2}\left(t, t^{\prime} ; \mathbf{p}\right) \rightarrow \frac{\left(n_{\mathbf{p}}(t)+1 / 2\right)^{2}}{2 \omega_{\mathbf{p}}^{2}(t)} \\
& \overline{\rho^{2}}\left(t, t^{\prime} ; \mathbf{p}\right) \rightarrow \frac{1}{2 \omega_{\mathbf{p}}^{2}(t)}
\end{aligned}
$$

Inserting these estimates in (288) for equal momenta yields

$$
\left[n_{\mathbf{p}}(t)+\frac{1}{2}\right]^{2} \gg \frac{3}{4} \quad \text { or } \quad n_{\mathbf{p}}(t) \gg 0.37 .
$$

This limit agrees rather well with what is obtained for the case of thermal equilibrium. For a Bose-Einstein distributed particle number $n_{\mathrm{BE}}=\left(e^{\omega / T}-1\right)^{-1}$ with temperature $T$ one finds $n_{\mathrm{BE}}(\omega=T)=0.58$, below which deviations from the classical thermal distribution become sizeable. We emphasize that in thermal equilibrium the fluctuationdissipation relation (129) ensures $\left|F^{(\text {eq })}(\omega, \mathbf{p})\right| \gg\left|\rho^{(\text {eq })}(\omega, \mathbf{p})\right|$ for high-temperature modes $T \gg \omega$ :

$$
F^{(\mathrm{eq})}(\omega, \mathbf{p})=-i\left(n_{\mathrm{BE}}(\omega)+\frac{1}{2}\right) \rho^{(\mathrm{eq})}(\omega, \mathbf{p}) \stackrel{T \geqq \omega}{\simeq}-i \frac{T}{\omega} \rho^{(\mathrm{eq})}(\omega, \mathbf{p}) .
$$


The nonequilibrium estimate (290) as well as the equilibrium result (291) leads one to expect that the quantum evolution described by $F$ and $\rho$ is well approximated by the classical evolution in terms of $F_{\mathrm{cl}}$ and $\rho_{c l}$ for large initial occupation numbers $n_{\mathbf{p}}(t=0)$. However, note that the nonequilibrium classicality condition (288) is typically not fulfilled at all times, since the unequal-time correlator $F\left(t, t^{\prime} ; \mathbf{p}\right)$ can oscillate around zero with a phase difference to the oscillations of $\rho\left(t, t^{\prime} ; \mathbf{p}\right)$ (cf. also the free-field expressions (211)). In particular, we will find that characteristic quantities for the quantum late-time behavior such as thermalization times are very badly approximated by the respective classical estimates. Nevertheless, for time-averaged quantities and not too late times $t \ll t_{\mathrm{eq}}$, i.e. before the approach to quantum thermal equilibrium sets in, the classical evolution can give an accurate description for sufficiently high initial occupation numbers as will be demonstrated below.

A similar analysis can be performed in the presence of a non-vanishing classical macroscopic field $\phi_{a, \mathrm{cl}}(x)$ defined in (259). For instance, to NLO in the 2 PI $1 / N$ expansion the classical macroscopic field is described by the evolution equation

$$
\begin{aligned}
&\left(\left[\square_{x}+\frac{\lambda}{6 N} \phi_{\mathrm{cl}}^{2}(x)\right]\right.\left.\delta_{a b}+M_{\mathrm{cl}, a b}^{2}\left(x ; \phi_{\mathrm{cl}} \equiv 0, F_{\mathrm{cl}}\right)\right) \phi_{\mathrm{cl}, b}(x) \\
&=-\int_{0}^{x^{0}} \mathrm{~d} y \Sigma_{\rho, a b}^{\mathrm{cl}}\left(x, y ; \phi_{\mathrm{cl}} \equiv 0, F_{\mathrm{cl}}, \rho_{\mathrm{cl}}\right) \phi_{\mathrm{cl}, b}(y)
\end{aligned}
$$

where $M_{\mathrm{cl}, a b}^{2}\left(x ; \phi_{\mathrm{cl}} \equiv 0, F_{\mathrm{cl}}\right)$ is given by (146) with the replacement $F \rightarrow F_{\mathrm{cl}}$, and $\Sigma_{\rho, a b}^{\mathrm{cl}}\left(x, y ; \phi_{\mathrm{cl}} \equiv 0, F_{\mathrm{cl}}, \rho_{\mathrm{cl}}\right)$ is obtained from the respective quantum self-energy $\Sigma_{\rho, a b}(x, y ; \phi \equiv 0, F, \rho)$ given in (185) by employing (285). This can be directly compared to the corresponding evolution equation (189) for the case of the quantum field theory.

\subsection{Precision tests and the role of quantum corrections}

The nonequilibrium evolution of classical correlation functions in the $O(N)$ model can be obtained numerically up to controlled statistical errors. Initial conditions for the nonequilibrium evolution are determined from a probability functional on classical phase-space. The subsequent time evolution is solved numerically using the classical equation of motion for the field (258). The results presented below have been obtained from sampling 50000-80000 independent initial conditions to approximate the exact evolution of correlation functions. The statistical propagator is constructed from these individual runs according to (261) and using (263) for the spectral function. Since these results include all orders in $1 / N$, they can be used for a precision test of the $2 \mathrm{PI} 1 / \mathrm{N}$ expansion implemented in classical statistical field theory. We emphasize that this compares two very different calculational procedures: the results from the simulation involve thousands of individual runs from which the correlators are constructed, while the corresponding results employing the $2 \mathrm{PI} 1 / N$ expansion involve only a single run solving directly the evolution equation for the correlators. The accuracy of the simulations manifests itself also in the fact that the time-reversal invariant dynamics can be explicitly reversed in practice for not too late times. The close agreement between full and 2PI 

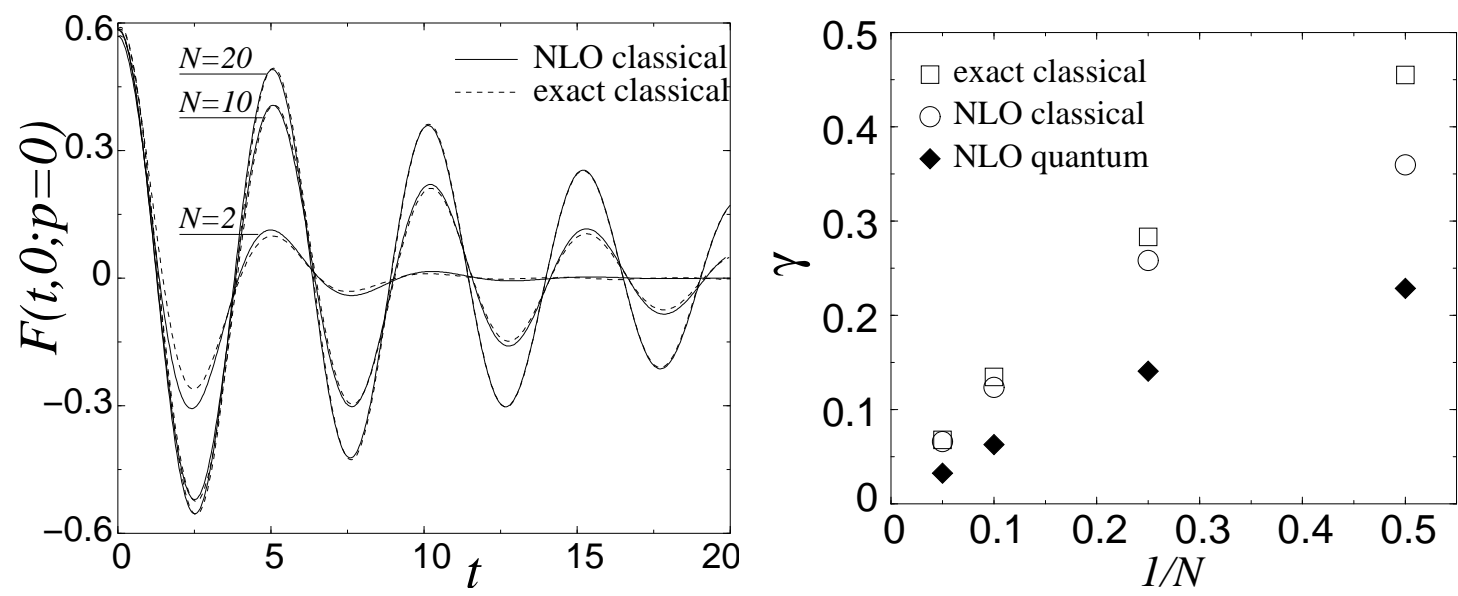

FIGURE 16. Left: Unequal-time two-point function $F(t, 0 ; p=0)$ at zero momentum for $N=2,10,20$. The full lines show results from the NLO classical evolution and the dashed lines from the exact classical evolution (MC). One observes a convergence of classical NLO and exact results already for moderate values of $N$. Right: Damping rate extracted from $F(t, 0 ; p=0)$ as a function of $1 / N$. Open symbols represent NLO and exact classical evolution. The quantum results are shown with full symbols for comparison. The initial conditions are characterized by low occupation numbers so that quantum effects become sizeable. One observes that in the quantum theory the damping rate is reduced compared to the classical theory. (All in units of $m_{R}$.)

NLO results, which will be observed below, is therefore also indicative of the numerical precision of the latter.

We consider a system that is invariant under space translations and work in momentum space. We choose a Gaussian initial state such that a specification of the initial two-point functions is sufficient. As mentioned above, the classical spectral function at initial time is completely determined from the equal-time relations (264). For the statistical propagator we take $F(0,0 ; p)=\left[n_{p}(0)+\frac{1}{2}\right] / \omega_{p}$, with the initial particle number representing a peaked "tsunami" (cf. Sect. 4.1.1).

The initial mode energy is given by $\omega_{p}=\left(p^{2}+M_{\mathrm{cl}}^{2}\right)^{1 / 2}$ where $M_{\mathrm{cl}}$ is the one-loop renormalized mass in presence of the nonequilibrium medium, determined from the oneloop "gap equation" for $M_{\mathrm{cl}}$ in Eq. (283). As a renormalization condition we choose the one-loop renormalized mass in vacuum $\left.m_{R} \equiv M\right|_{n(0)=0}=1$ as our dimensionful scale. The results shown below are obtained using a fixed coupling constant $\lambda / m_{R}^{2}=30$.

On the left of Fig. 16 the classical statistical propagator $F_{\mathrm{cl}}(t, 0 ; p=0)$ is presented for three values of $N$. All other parameters are kept constant. The figure compares the time evolution using the $2 \mathrm{PI} 1 / N$ expansion to NLO and the full Monte Carlo (MC) calculation. One observes that the approximate time evolution of the correlation function shows a rather good agreement with the exact result even for small values of $N$ (note that the effective four-point coupling is strong, $\lambda / 6 N=2.5 m_{R}^{2}$ for $N=2$ ). For $N=20$ the exact and NLO evolution can hardly be distinguished. A very sensitive quantity for comparisons is the damping rate $\gamma$, which is obtained from an exponential fit to the envelope of $F_{\mathrm{cl}}(t, 0 ; p=0)$. The systematic convergence of the NLO and the Monte Carlo result as a function of $1 / N$ can be observed from the right graph of Fig. 16. The quantitatively accurate description of far-from-equilibrium processes within the NLO 
approximation of the 2PI effective action is manifest.

The right graph of Fig. 16 also shows the damping rate from the quantum evolution, using the same initial conditions and parameters. One observes that the damping in the quantum theory differs and, in particular, is reduced compared to the classical result. The effective loss of details about the initial conditions takes more time for the quantum system than for the corresponding classical one. In the limit $N \rightarrow \infty$ damping of the unequal-time correlation function goes to zero since the nonlocal part of the selfenergies vanishes identically at LO large- $N$ and scattering is absent. In this limit there is no difference between evolution in a quantum and classical statistical field theory. (Cf. Sec. 4.1.1 and the discussion above.)

For finite $N$ scattering is present and quantum and classical evolution differ in general. However, as discussed in Sec. 5.2, the classical field approximation may be expected to become a reliable description for the quantum theory if the number of field quanta in each field mode is sufficiently high. We observe that increasing the initial particle number density leads to a convergence of quantum and classical time evolution at not too late times. In Fig. 17 (left) the time evolution of the equal-time correlation function $F(t, t ; p)$ is shown for several momenta $p$ and $N=10$. Here the integrated particle density $\int \frac{d p}{2 \pi} n_{p}(0) / M_{\mathrm{cl}}=1.2$ is six times as high as in Fig. 16. At $p=2 p_{\mathrm{ts}}$ one finds $n_{p=2 p_{\text {ts }}}(0) \simeq 0.35$ and a slightly larger value at this momentum of about $\simeq 0.5$ for late times. For these initial conditions the estimate (290) for the classicality condition (288) is therefore approximately valid up to momenta $p \simeq 2 p_{\mathrm{ts}}$, and one indeed observes from the left of Fig. 17 a rather good agreement of quantum and classical evolution in this range. For an estimate of the NLO truncation error we also give the full (MC) result for $N=10$ showing a quantitative agreement with the classical NLO evolution both at early and later times.

\subsubsection{Classical equilibration and quantum thermal equilibrium}

From the left of Fig. 17 one observes that the initially highly occupied "tsunami" modes "decay" as time proceeds and the low momentum modes become more and more populated. At late times the classical theory and the quantum theory approach their respective equilibrium distributions. Since classical and quantum thermal equilibrium are distinct, the classical and quantum time evolutions have to deviate at sufficiently late times. Figure 17 shows the time dependent inverse slope parameter

$$
T(t, p) \equiv-n_{p}(t)\left[n_{p}(t)+1\right]\left(\frac{d n_{p}}{d \varepsilon_{p}}\right)^{-1},
$$

which has been introduced in Sec. 4.1.2 to study the approach to quantum thermal equilibrium. ${ }^{34}$ It employs the effective particle number $n_{p}(t)$ defined in $(201)^{35}$ and mode energy $\varepsilon_{p}(t)$ given by (203). Initially one observes a very different behavior of

\footnotetext{
34 Note that $d \log \left(n_{p}^{-1}(t)+1\right) / d \varepsilon_{p}(t)=T^{-1}(t, p)$.

35 Here we employ $Q\left(t, t^{\prime} ; \mathbf{p}\right)=0$, cf. Sec. 4.1.2.
} 

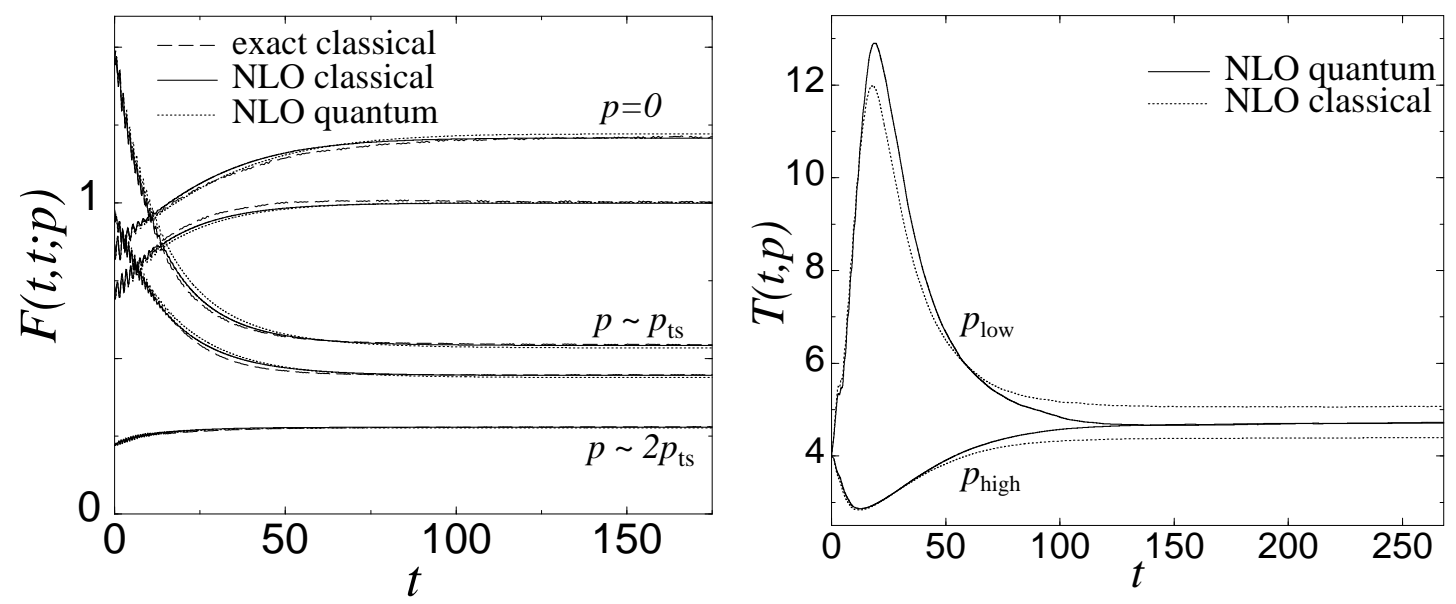

FIGURE 17. Left: Nonequilibrium evolution of the equal-time two-point function $F(t, t ; p)$ for $N=10$ for various momenta $p$. One observes a good agreement between the exact MC (dashed) and the NLO classical result (full). The quantum evolution is shown with dotted lines. The integrated initial particle density is six times as high as in Fig. 16. Right: A very sensitive quantity to study deviations is the time dependent inverse slope $T(t, p)$ defined in the text. When quantum thermal equilibrium with a BoseEinstein distributed particle number is approached all modes get equal $T(t, p)=T_{\text {eq }}$, as can be observed to high accuracy for the quantum evolution. For classical thermal equilibrium the defined inverse slope remains momentum dependent.

$T(t, p)$ for the low and high momentum modes, indicating that the system is far from equilibrium. The quantum evolution approaches quantum thermal equilibrium with a momentum independent inverse slope $T_{\mathrm{eq}}=4.7 m_{R}$ to high accuracy. In contrast, in the classical limit the slope parameter remains momentum dependent since the classical dynamics does of course not reach a Bose-Einstein distribution.

To see this in more detail we note that for a Bose-Einstein distribution, $n_{\mathrm{BE}}\left(\varepsilon_{p}\right)=$ $1 /\left[\exp \left(\varepsilon_{p} / T_{\mathrm{eq}}\right)-1\right]$, the inverse slope (293) is independent of the mode energies and equal to the temperature $T_{\text {eq }}$. During the nonequilibrium evolution effective thermalization can therefore be observed if $T(t, p)$ becomes time and momentum independent, $T(t, p) \rightarrow T_{\mathrm{eq}}$. This is indeed seen on the left of Fig. 17 for the quantum system. If the system is approaching classical equilibrium at some temperature $T_{\mathrm{cl}}$ and is weakly coupled, the following behavior is expected. From the definition (201) of $n_{p}(t)$ in terms in two-point functions, we expect to find approximately

$$
T(t, p) \rightarrow T_{\mathrm{cl}}\left(1-\varepsilon_{p}^{2} / T_{\mathrm{cl}}^{2}\right),
$$

i.e. a remaining momentum dependence with $T(t, p)<T\left(t, p^{\prime}\right)$ if $\varepsilon_{p}>\varepsilon_{p}^{\prime}$. Indeed, this is what one observes for the classical field theory result in Fig. 17.

For a classical theory a very simple test for effective equilibration is available. An exact criterion can be obtained from the classical counterpart of the "KMS" condition for thermal equilibrium discussed in Sec. 3.4.2. In coordinate space the classical equilibrium "KMS" condition reads

$$
\frac{1}{T_{\mathrm{cl}}} \frac{\partial}{\partial x^{0}} F_{\mathrm{cl}}^{(\mathrm{eq})}(x-y)=-\rho_{\mathrm{cl}}^{(\mathrm{eq})}(x-y),
$$




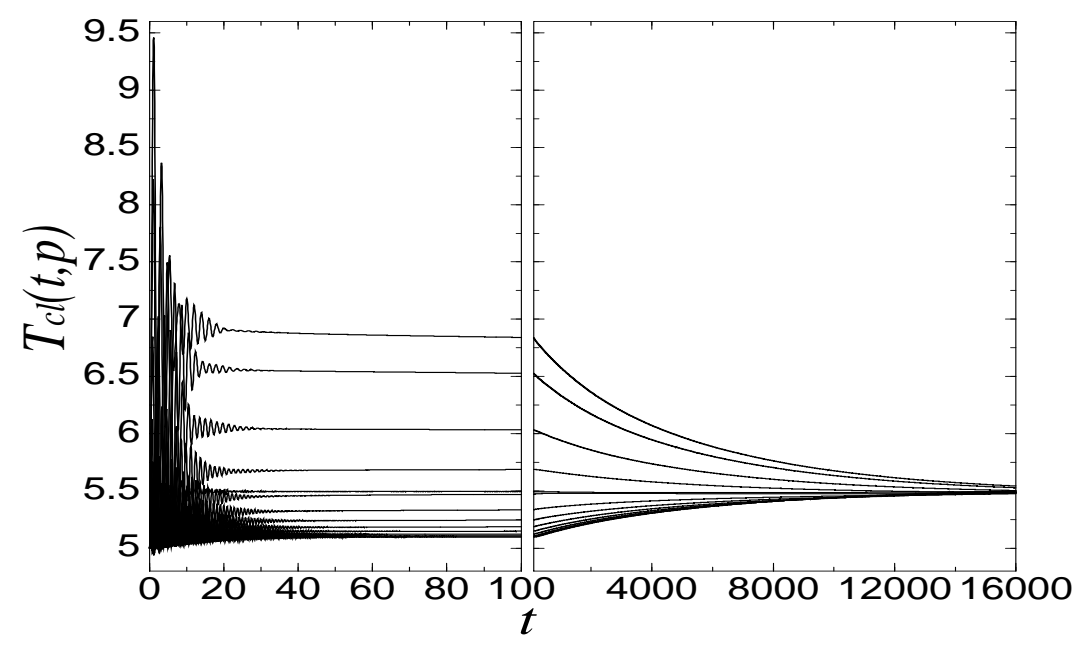

FIGURE 18. Nonequilibrium time evolution in $1+1$ dimensions in the classical limit of the threeloop approximation of the 2PI effective action for one scalar field, $N=1$. Shown is the effective mode temperature $T_{\mathrm{cl}}(t, p)$, defined in the text, with initial $T_{\mathrm{cl}}(0, p) / m_{R}=5$ for all $p$. The classical field theory approaches classical equilibrium, $T_{\mathrm{cl}}(t, p) \rightarrow T_{\mathrm{cl}}$, after a very long time.

and in momentum space

$$
F_{\mathrm{cl}}^{(\mathrm{eq})}(k)=-i n_{\mathrm{cl}}\left(k^{0}\right) \rho_{\mathrm{cl}}^{(\mathrm{eq})}(k), \quad n_{\mathrm{cl}}\left(k^{0}\right)=\frac{T_{\mathrm{cl}}}{k^{0}} .
$$

Differentiating Eq. (295) with respect to $y^{0}$ at $x^{0}=y^{0}=t$ gives

$$
\left.\frac{1}{T_{\mathrm{cl}}} \frac{\partial}{\partial y^{0}} \frac{\partial}{\partial x^{0}} F_{\mathrm{cl}}^{(\mathrm{eq})}(x-y)\right|_{x^{0}=y^{0}=t}=-\left.\frac{\partial}{\partial y^{0}} \rho_{\mathrm{cl}}^{(\mathrm{eq})}(x-y)\right|_{x^{0}=y^{0}=t} .
$$

Combining this KMS relation with the equal-time condition (264) for the spectral function leads to

$$
\left.\partial_{t} \partial_{t^{\prime}} F_{\mathrm{cl}}^{(\mathrm{eq})}\left(t, t^{\prime} ; \mathbf{x}-\mathbf{y}\right)\right|_{t=t^{\prime}}=T_{\mathrm{cl}} \delta(\mathbf{x}-\mathbf{y}) .
$$

In terms of the fluctuating classical fields $\pi_{a}(x) \equiv \partial_{x^{0}} \varphi_{a}(x)$ this is of course the wellknown equilibrium relation $\left\langle\pi_{a}(t, \mathbf{x}) \pi_{b}(t, \mathbf{y})\right\rangle_{\mathrm{cl}}^{(\mathrm{eq})}=T_{\mathrm{cl}} \delta(\mathbf{x}-\mathbf{y}) \delta_{a b}$. Out of equilibrium one can define an effective classical mode temperature

$$
T_{\mathrm{cl}}(t, p)=\left.\partial_{t} \partial_{t^{\prime}} F_{\mathrm{cl}}\left(t, t^{\prime} ; p\right)\right|_{t=t^{\prime}}
$$

and effective classical equilibration is observed if $T_{\mathrm{cl}}(t, p)$ becomes time and momentum independent, $T_{\mathrm{cl}}(t, p) \rightarrow T_{\mathrm{cl}}$.

Apart from the 2PI 1/ $N$ expansion the late-time behavior can also be studied from the 2PI loop expansion. For the quantum theory this has been demonstrated in Sec. 4.1.4 for the three-loop approximation of the 2PI effective action (cf. Fig. 6, left). The equivalent approximation in the classical theory is given by the evolution equations (280) and (281) with the two-loop self-energies (286). In order to demonstrate this, we will use the loop approximation to verify explicitly the above statements about classical equilibration. 
In Fig. 18 the nonequilibrium evolution of the effective "mode temperature" $T_{\mathrm{cl}}(t, p)$ is shown for various momentum modes in the $(1+1)$-dimensional classical scalar field theory for $N=1$. The equations are solved by a lattice discretization with spatial lattice spacing $m_{R} a=0.4$, time step $a_{0} / a=0.2$, and $N_{s}=24$ sites. Without loss of generality we use $\lambda / m_{R}^{2}=1$. For the initial ensemble we take $F_{\mathrm{cl}}(0,0 ; p)=T_{0} /\left(p^{2}+m_{R}^{2}\right)$ and $\left.\partial_{t} \partial_{t^{\prime}} F_{\mathrm{cl}}\left(t, t^{\prime} ; p\right)\right|_{t=t^{\prime}=0}=T_{0}$ with $T_{0} / m_{R}=5$. We have observed that at sufficiently late times the contributions from early times to the dynamics are effectively suppressed. This fact has been employed in Fig. 18 to reach the very late times. One sees that at sufficiently late times the system relaxes towards classical equilibrium with a final temperature $T_{\mathrm{cl}} / m_{R} \approx 5.5$. For the zero-momentum mode we find an exponential latetime relaxation of $T_{\mathrm{cl}}(t, p=0)$ towards $T_{\mathrm{cl}}$ with a small rate $\left(\sim 2 \times 10^{-4} m_{R}\right)$. It should be emphasized that typical classical equilibration times are substantially larger than the times required to approach thermal equilibrium in the respective quantum theory. In contrast to the quantum theory statements about equilibration times are, however, in general not insensitive to the employed momentum regularization for the classical theory because of the classical Rayleigh-Jeans divergences.

\section{6. $N$-PARTICLE IRREDUCIBLE GENERATING FUNCTIONALS II: EQUIVALENCE HIERARCHY}

To understand the success and, more importantly, the limitations of expansion schemes based on the 2 PI effective action we consider in this section $n$-particle irreducible ( $n \mathrm{PI})$ effective actions for $n>2$. Recall that the description of the 2PI effective action $\Gamma[\phi, D]$ employs a self-consistently dressed one-point function $\phi$ and two-point function, which for notational purposes we denote here by $D:^{36}$ The one-point and two-point functions are dressed by solving the equations of motion $\delta \Gamma / \delta \phi=0$ and $\delta \Gamma / \delta D=0$ for a given order in the (e.g. loop) expansion of $\Gamma[\phi, D]$ (cf. Sec. 2). However, the 2PI effective action does not treat the higher $n$-point functions with $n>2$ on the same footing as the lower ones: The three- and four-point function etc. are not self-consistently dressed in general, i.e. the corresponding proper three-vertex $V_{3}$ and four-vertex $V_{4}$ are given by the classical ones. In contrast, the $n \mathrm{PI}$ effective action $\Gamma\left[\phi, D, V_{3}, V_{4}, \ldots, V_{n}\right]$ provides a dressed description for the proper vertices $V_{3}, V_{4}, \ldots, V_{n}$ as well, with $\delta \Gamma / \delta V_{3}=$ $0, \delta \Gamma / \delta V_{4}=0, \ldots, \delta \Gamma / \delta V_{n}=0$.

The use of $n$ PI effective actions with higher $n>2$ is not entirely academic. They are relevant in the presence of initial-time sources describing a non-Gaussian initial density matrix for nonequilibrium evolutions (cf. the discussion in Secs. 3.2 and 3.3). They are also known to be relevant in high-temperature gauge theories for a quantitative description of transport coefficients in the context of kinetic theory. As an example, the calculation of shear viscosity in a theory like QCD can be based on the inclusion of an infinite series of 2PI "ladder" diagrams in order to recover the leading order "on-shell" results in the gauge coupling $g$. Further examples where approximation schemes based

${ }^{36} G$ will denote the ghost propagator in gauge theories below. 
on higher $n$ PI effective actions are relevant include critical phenomena near second-order phase transitions. For instance, the quantitative description of the universal behavior near the second-order phase transition of scalar $\phi^{4}$ theory goes beyond a 2PI loop expansion. ${ }^{37}$ It requires taking into account vertex corrections that start with the 4PI effective action to four-loop order. The latter agrees with the most general $n$ PI loop expansion to that order, which is shown below.

The evolution equations, which are obtained by variation of the $n$ PI effective action, are closely related to known exact identities for correlation functions, i.e. SchwingerDyson (SD) equations. Without approximations the equations of motion obtained from an exact $n$ PI effective action and the exact SD equations have to agree since one can always map identities onto each other. However, in general this is no longer the case for a given order in the loop or coupling expansion of the $n \mathrm{PI}$ effective action. By construction, SD equations are expressed in terms of loop diagrams including both classical and dressed vertices, which leads to ambiguities of whether classical or dressed ones should be employed at a given truncation order. In particular, SD equations are not closed a priori in the sense that the equation for a given $n$-point function always involves information about $m$-point functions with $m>n$. These problems are absent using effective action techniques. In turn, the $n$ PI results can be used to resolve ambiguities of whether classical or dressed vertices should be employed for a given truncation of a SD equation. For instance, in QCD the three-loop effective action result leads to evolution equations, which are equivalent to the SD equation for the two-point function and the one-loop three-point function if all vertices in loop-diagrams for the latter are replaced by the full vertices at that order. As mentioned in previous sections, the "conserving" property of using an effective action truncation can have important advantages, in particular if applied to nonequilibrium time evolution problems, where the presence of basic constants of motion such as energy conservation is crucial.

We will derive below the 4PI effective action for a nonabelian $S U(N)$ gauge theory with fermions up to four-loop or $\mathscr{O}\left(g^{6}\right)$ corrections, starting from the 2PI effective action and doing subsequent Legendre transforms (Secs. 6.1 and 6.2). The class of models include gauge theories such as QCD or abelian theories as QED, as well as simple scalar field theories with cubic or quartic interactions. In Sec. 6.1.2 we derive an equivalence hierarchy for $n \mathrm{PI}$ effective actions, which implies that the 4PI results to this order are equivalent to those from the $n \mathrm{PI}$ effective action up to four-loop or $\mathscr{O}\left(g^{6}\right)$ corrections for arbitrary $n>4$. We derive the non-equilibrium gauge field and fermion evolution equations (Sec. 6.2.3), and discuss the connection to kinetic theory in Sec. 6.3.

37 Critical phenomena can be described using the $1 / N$ expansion of the 2 PI effective action beyond leading order. These approximations indeed resum an infinite number of loop diagrams and, for instance, the NLO result can be rewritten as a "loop approximation in the presence of an effective four-vertex" (cf. Secs. 2.1 and 4.3). 


\subsection{Higher effective actions}

Recall that all information about the quantum theory can be obtained from the effective action, which is a generating functional for Green's functions evaluated in the absence of external sources, i.e. at the physical or stationary point. All functional representations of the effective action are equivalent in the sense that they are generating functionals for Green's functions including all quantum/statistical fluctuations and, in the absence of sources, have to agree by construction: ${ }^{38}$

$$
\Gamma[\phi]=\Gamma[\phi, D]=\Gamma\left[\phi, D, V_{3}\right]=\Gamma\left[\phi, D, V_{3}, V_{4}\right]=\ldots=\Gamma\left[\phi, D, V_{3}, V_{4}, \ldots, V_{n}\right]
$$

for arbitrary $n$ without further approximations. However, e.g. loop expansions of the 1PI effective action to a given order in the presence of the "background" field $\phi$ differ in general from a loop expansion of $\Gamma[\phi, D]$ in the presence of $\phi$ and $D$. A similar statement can be made for expansions of higher functional representations. As mentioned in the introduction in Sec. 1.1.2, for applications it is often desirable to obtain a selfconsistently complete description, which to a given order in the expansion determines $\Gamma\left[\phi, D, V_{3}, V_{4}, \ldots, V_{n}\right]$ for arbitrarily high $n$. For this it is important to realize that there exists an equivalence hierarchy as displayed in the introduction in Eq. (13), which is derived in Sec. 6.1.2. For instance at three-loop order one has:

$$
\begin{aligned}
\Gamma^{(3 \text { loop })}[\phi] \neq \Gamma^{(3 \text { loop })}[\phi, D] \neq \Gamma^{(3 \text { loop })}\left[\phi, D, V_{3}\right] & =\Gamma^{(3 \text { loop })}\left[\phi, D, V_{3}, V_{4}\right] \\
& =\Gamma^{(31 \text { loop })}\left[\phi, D, V_{3}, V_{4}, \ldots, V_{n}\right],
\end{aligned}
$$

for arbitrary $n>4$ in the absence of sources. As a consequence, there is no difference between $\Gamma^{(3 \text { loop })}\left[\phi, D, V_{3}\right]$ and $\Gamma^{(3 \text { loop })}\left[\phi, D, V_{3}, V_{4}\right]$ etc. at the stationary point, and the 3PI effective action captures already the complete answer for the self-consistent description to this order. More explicitly, the equality reads $\Gamma^{(310 o p)}\left[\phi, D(\phi), V_{3}(\phi)\right]=$ $\Gamma^{(3 \text { loop })}\left[\phi, D(\phi), V_{3}(\phi), V_{4}(\phi)\right]=\ldots$ since at the stationary point of the effective action all $n$-point correlations become functions of the field expectation value $\phi$. At four-loop order the 4PI effective action would become relevant etc. For instance, for a theory as quantum electrodynamics (QED) or chromodynamics (QCD) the 2PI effective action provides a self-consistently complete description to two-loop order or $^{39} \mathscr{O}\left(g^{2}\right)$ : For a two-loop approximation all $n$ PI descriptions with $n \geq 2$ are equivalent in the absence of sources. In contrast, a self-consistently complete result to three-loop order or $\mathscr{O}\left(g^{4}\right)$ requires at least the 3PI effective action etc. To go to much higher loop-order can become somewhat academic from the point of view of calculational feasibility.

To present the argument we will first consider the 4PI effective action for a simple generic scalar model with cubic and quartic interactions. The formal generalization to

\footnotetext{
38 Of course, the nonequilibrium $n$ PI effective action in the presence of initial-time sources representing an initial density matrix can differ in general. However, these pose no additional complications since they vanish identically for times different than the initial time. Cf. the discussions in Secs. 3.3 and 3.4.3.

${ }^{39}$ Here, and throughout the paper, $g$ means the strong gauge coupling $g_{s}$ for QCD, while it should be understood as the electric charge $e$ for QED. For the power counting we take $\phi \sim \mathscr{O}(1 / g)$ (cf. Sec. 6.1.1). The metric is denoted as $g^{\mu \nu}=g_{\mu \nu}=\operatorname{diag}(1,-1,-1,-1)$.
} 
fermionic and gauge fields is straightforward, and in Sec. 6.2 the construction is done for $S U(N)$ gauge theories with fermions. We use here a concise notation where Latin indices represent all field attributes, numbering real field components and their internal as well as space-time labels, and sum/integration over repeated indices is implied. We consider the classical action

$$
S[\varphi]=\frac{1}{2} \varphi_{i} i D_{0, i j}^{-1} \varphi_{j}-\frac{g}{3 !} V_{03, i j k} \varphi_{i} \varphi_{j} \varphi_{k}-\frac{g^{2}}{4 !} V_{04, i j k l} \varphi_{i} \varphi_{j} \varphi_{k} \varphi_{l},
$$

where we scaled out a coupling constant $g$ for later convenience. The generating functional for Green's functions in the presence of quadratic, cubic and quartic source terms is given by:

$$
\begin{aligned}
Z\left[J, R, R_{3}, R_{4}\right]= & \exp \left(i W\left[J, R, R_{3}, R_{4}\right]\right) \\
= & \int \mathscr{D} \varphi \exp \left\{i \left(S[\varphi]+J_{i} \varphi_{i}+\frac{1}{2} R_{i j} \varphi_{i} \varphi_{j}\right.\right. \\
& \left.\left.+\frac{1}{3 !} R_{3, i j k} \varphi_{i} \varphi_{j} \varphi_{k}+\frac{1}{4 !} R_{4, i j k l} \varphi_{i} \varphi_{j} \varphi_{k} \varphi_{l}\right)\right\} .
\end{aligned}
$$

The generating functional for connected Green's functions, $W$, can be used to define the connected two-point $(D)$, three-point $\left(D_{3}\right)$ and four-point function $\left(D_{4}\right)$ in the presence of the sources,

$$
\begin{aligned}
\frac{\delta W}{\delta J_{i}}= & \phi_{i} \\
\frac{\delta W}{\delta R_{i j}}= & \frac{1}{2}\left(D_{i j}+\phi_{i} \phi_{j}\right) \\
\frac{\delta W}{\delta R_{3, i j k}}= & \frac{1}{6}\left(D_{3, i j k}+D_{i j} \phi_{k}+D_{k i} \phi_{j}+D_{j k} \phi_{i}+\phi_{i} \phi_{j} \phi_{k}\right) \\
\frac{\delta W}{\delta R_{4, i j k l}}= & \frac{1}{24}\left(D_{4, i j k l}+\left[D_{3, i j k} \phi_{l}+3 \text { perm. }\right]+\left[D_{i j} D_{k l}+2 \text { perm. }\right]\right. \\
& \left.+\left[D_{i j} \phi_{k} \phi_{l}+5 \text { perm. }\right]+\phi_{i} \phi_{j} \phi_{k} \phi_{l}\right) .
\end{aligned}
$$

We denote the proper three-point and four-point vertices by $g V_{3}$ and $g^{2} V_{4}$, respectively, and define

$$
\begin{aligned}
D_{3, i j k}= & -i g D_{i i^{\prime}} D_{j j^{\prime}} D_{k k^{\prime}} V_{3, i^{\prime} j^{\prime} k^{\prime}} \\
D_{4, i j k l}= & -i g^{2} D_{i i^{\prime}} D_{j j^{\prime}} D_{k k^{\prime}} D_{l l^{\prime}} V_{4, i^{\prime} j^{\prime} k^{\prime} l^{\prime}} \\
& +g^{2}\left(D_{i i^{\prime}} D_{j j^{\prime}} D_{k^{\prime} u^{\prime}} D_{w^{\prime} l} D_{v^{\prime} k}+D_{i i^{\prime}} D_{j^{\prime} u^{\prime}} D_{k^{\prime} l} D_{j v^{\prime}} D_{w^{\prime} k}\right. \\
& \left.+D_{i i^{\prime}} D_{j^{\prime} u^{\prime}} D_{k^{\prime} k} D_{j v^{\prime}} D_{l^{\prime} l}\right) V_{3, i^{\prime} j^{\prime} k^{\prime}} V_{3, u^{\prime} v^{\prime} w^{\prime}} .
\end{aligned}
$$


The effective action is obtained as the Legendre transform of $W\left[J, R, R_{3}, R_{4}\right]: 40$

$$
\begin{aligned}
\Gamma\left[\phi, D, V_{3}, V_{4}\right]= & W-\frac{\delta W}{\delta J_{i}} J_{i}-\frac{\delta W}{\delta R_{i j}} R_{i j} \\
& -\frac{\delta W}{\delta R_{3, i j k}} R_{3, i j k}-\frac{\delta W}{\delta R_{4, i j k l}} R_{4, i j k l} .
\end{aligned}
$$

For vanishing sources one observes from (310) the stationarity conditions

$$
\frac{\delta \Gamma}{\delta \phi}=\frac{\delta \Gamma}{\delta D}=\frac{\delta \Gamma}{\delta V_{3}}=\frac{\delta \Gamma}{\delta V_{4}}=0,
$$

which provide the equations of motion for $\phi, D, V_{3}$ and $V_{4}$.

\subsubsection{PI effective action up to four-loop order corrections}

Since the Legendre transforms employed in (310) can be equally performed subsequently, a most convenient computation of $\Gamma\left[\phi, D, V_{3}, V_{4}\right]$ starts from the $2 \mathrm{PI}$ effective action $\Gamma[\phi, D]$. According to (28) the exact 2 PI effective action can be written as:

$$
\Gamma[\phi, D]=S[\phi]+\frac{i}{2} \operatorname{Tr} \ln D^{-1}+\frac{i}{2} \operatorname{Tr} D_{0}^{-1}(\phi) D+\Gamma_{2}[\phi, D]+\text { const },
$$

with the field-dependent inverse classical propagator

$$
i D_{0}^{-1}(\phi)=\frac{\delta^{2} S[\phi]}{\delta \phi \delta \phi} .
$$

To simplify the presentation, we use in the following a symbolic notation which suppresses indices and summation or integration symbols (suitably regularized). In this no-

${ }^{40}$ In terms of the standard one-particle irreducible effective action $\Gamma[\phi]=W[J]-J \phi$ the proper vertices $V_{3}$ and $V_{4}$ are given by

$$
g V_{3}=-\frac{\delta^{3} \Gamma[\phi]}{\delta \phi \delta \phi \delta \phi} \quad, \quad g^{2} V_{4}=-\frac{\delta^{4} \Gamma[\phi]}{\delta \phi \delta \phi \delta \phi \delta \phi} .
$$

Here it is useful to note that in terms of the connected Green's functions $D_{n}$ one has

$$
\begin{aligned}
\frac{\delta^{2} W[J]}{\delta J \delta J} & =i D \quad, \quad \frac{\delta^{2} \Gamma[\phi]}{\delta \phi \delta \phi}=i D^{-1} \\
\frac{\delta^{3} W[J]}{\delta J \delta J \delta J} & =-D_{3}=-i D^{3} \frac{\delta^{3} \Gamma[\phi]}{\delta \phi \delta \phi \delta \phi} . \\
\frac{\delta^{4} W[J]}{\delta J \delta J \delta J \delta J} & =-i D_{4}=D^{4} \frac{\delta^{4} \Gamma[\phi]}{\delta \phi \delta \phi \delta \phi \delta \phi}+3 i D^{5}\left(\frac{\delta^{3} \Gamma[\phi]}{\delta \phi \delta \phi \delta \phi}\right)^{2} .
\end{aligned}
$$


tation the inverse classical propagator reads

$$
i D_{0}^{-1}(\phi)=i D_{0}^{-1}-g \phi V_{03}-\frac{1}{2} g^{2} \phi^{2} V_{04},
$$

and to three-loop order one has ${ }^{41}$ (cf. Sec. 2.1)

$$
\begin{aligned}
\Gamma_{2}[\phi, D]= & -\frac{1}{8} g^{2} D^{2} V_{04}+\frac{i}{12} D^{3}\left(g V_{03}+g^{2} \phi V_{04}\right)^{2}+\frac{i}{48} g^{4} D^{4} V_{04}^{2} \\
& +\frac{1}{8} g^{2} D^{5}\left(g V_{03}+g^{2} \phi V_{04}\right)^{2} V_{04}-\frac{i}{24} D^{6}\left(g V_{03}+g^{2} \phi V_{04}\right)^{4} \\
& +\mathscr{O}\left(\left.g^{n}\left(g^{2} \phi\right)^{m}\right|_{n+m=6}\right)
\end{aligned}
$$

for $n, m=0, \ldots, 6$. We emphasize that the exact $\phi$-dependence of $\Gamma_{2}[\phi, D]$ can be written as a function of the combination $\left(g V_{03}+g^{2} \phi V_{04}\right)$. In order to obtain the vertex 2PI effective action $\Gamma\left[\phi, D, V_{3}, V_{4}\right]$ from $\Gamma[\phi, D]$, one can exploit that the cubic and quartic source terms $\sim R_{3}$ and $\sim R_{4}$ appearing in (303) can be conveniently combined with the vertices $g V_{03}$ and $g^{2} V_{04}$ by the replacement:

$$
g V_{03} \rightarrow g V_{03}-R_{3} \equiv g \tilde{V}_{3} \quad, \quad g^{2} V_{04} \rightarrow g^{2} V_{04}-R_{4} \equiv g^{2} \tilde{V}_{4} .
$$

The 2PI effective action with the modified interaction is given by

$$
\Gamma_{\tilde{V}}[\phi, D]=W\left[J, R, R_{3}, R_{4}\right]-\frac{\delta W}{\delta J} J-\frac{\delta W}{\delta R} R .
$$

Since

$$
\frac{\delta \Gamma_{\tilde{V}}}{\delta R_{3}}=\frac{\delta W}{\delta R_{3}} \quad, \quad \frac{\delta \Gamma_{\tilde{V}}}{\delta R_{4}}=\frac{\delta W}{\delta R_{4}},
$$

one can express the remaining Legendre transforms, leading to $\Gamma\left[\phi, D, V_{3}, V_{4}\right]$, in terms of the vertices $\tilde{V}_{3}, \tilde{V}_{4}$ and $V_{03}, V_{04}$ :

$$
\begin{aligned}
& \Gamma\left[\phi, D, V_{3}, V_{4}\right]=\Gamma_{\tilde{V}}[\phi, D]-\frac{\delta \Gamma_{\tilde{V}}[\phi, D]}{\delta R_{3}} R_{3}-\frac{\delta \Gamma_{\tilde{V}}[\phi, D]}{\delta R_{4}} R_{4} \\
& =\Gamma_{\tilde{V}}[\phi, D]-\frac{\delta \Gamma_{\tilde{V}}[\phi, D]}{\delta \tilde{V}_{3}}\left(\tilde{V}_{3}-V_{03}\right)-\frac{\delta \Gamma_{\tilde{V}}[\phi, D]}{\delta \tilde{V}_{4}}\left(\tilde{V}_{4}-V_{04}\right) .
\end{aligned}
$$

What remains to be done is expressing $\tilde{V}_{3}$ and $\tilde{V}_{4}$ in terms of $V_{3}$ and $V_{4}$. On the one hand, from (307) and the definitions (308) and (309) one has

$$
\begin{aligned}
\frac{\delta \Gamma_{\tilde{V}}[\phi, D]}{g \delta \tilde{V}_{3}}= & -\frac{1}{6}\left(-i g D^{3} V_{3}+3 D \phi+\phi^{3}\right), \\
\frac{\delta \Gamma_{\tilde{V}}[\phi, D]}{g^{2} \delta \tilde{V}_{4}}= & -\frac{1}{24}\left(-i g^{2} D^{4} V_{4}-3 g^{2} D^{5} V_{3}^{2}-4 i g D^{3} V_{3} \phi+3 D^{2}\right. \\
& \left.+6 D \phi^{2}+\phi^{4}\right) .
\end{aligned}
$$

\footnotetext{
41 Note that for $\phi \neq 0$, in the phase with spontaneous symmetry breaking, $\phi \sim \mathscr{O}(1 / g)$, and the three-loop result (315) takes into account the contributions up to order $g^{6}$.
} 
On the other hand, from the expansion of the 2PI effective action to three-loop order with (315) one finds ${ }^{42}$

$$
\begin{aligned}
\frac{\delta \Gamma_{\tilde{V}}[\phi, D]}{g \delta \tilde{V}_{3}}= & -\frac{1}{6} \phi^{3}-\frac{1}{2} D \phi+\frac{i}{6} D^{3}\left(g \tilde{V}_{3}+g^{2} \phi \tilde{V}_{4}\right) \\
& +\frac{1}{4} g^{2} D^{5}\left(g \tilde{V}_{3}+g^{2} \phi \tilde{V}_{4}\right) \tilde{V}_{4}-\frac{i}{6} D^{6}\left(g \tilde{V}_{3}+g^{2} \phi \tilde{V}_{4}\right)^{3} \\
& +\mathscr{O}\left(\left.g^{n}\left(g^{2} \phi\right)^{m}\right|_{n+m=5}\right) \\
\frac{\delta \Gamma_{\tilde{V}}[\phi, D]}{g^{2} \delta \tilde{V}_{4}}= & -\frac{1}{24} \phi^{4}-\frac{1}{4} D \phi^{2}-\frac{1}{8} D^{2}+\frac{i}{6} D^{3} \phi\left(g \tilde{V}_{3}+g^{2} \phi \tilde{V}_{4}\right) \\
& +\frac{i}{24} g^{2} D^{4} \tilde{V}_{4}+\frac{1}{4} g^{2} D^{5} \phi\left(g \tilde{V}_{3}+g^{2} \phi \tilde{V}_{4}\right) \tilde{V}_{4} \\
& +\frac{1}{8} D^{5}\left(g \tilde{V}_{3}+g^{2} \phi \tilde{V}_{4}\right)^{2}-\frac{i}{6} D^{6} \phi\left(g \tilde{V}_{3}+g^{2} \phi \tilde{V}_{4}\right)^{3} \\
& +\mathscr{O}\left(\left.g^{n-2}\left(g^{2} \phi\right)^{m}\right|_{n+m=6}\right) .
\end{aligned}
$$

Comparing (322) and (320) yields

$$
\begin{aligned}
g V_{3}= & \left(g \tilde{V}_{3}+g^{2} \phi \tilde{V}_{4}\right)-\frac{3}{2} i g^{2} D^{2}\left(g \tilde{V}_{3}+g^{2} \phi \tilde{V}_{4}\right) \tilde{V}_{4}-D^{3}\left(g \tilde{V}_{3}+g^{2} \phi \tilde{V}_{4}\right)^{3} \\
& +\mathscr{O}\left(\left.g^{n}\left(g^{2} \phi\right)^{m}\right|_{n+m=5}\right) .
\end{aligned}
$$

Similarly, for $V_{4}$ comparing (323) and (321), and using (324) one finds

$$
g^{2} V_{4}=g^{2} \tilde{V}_{4}+\mathscr{O}\left(\left.g^{n-2}\left(g^{2} \phi\right)^{m}\right|_{n+m=6}\right) .
$$

This can be used to invert the above relations as

$$
\begin{aligned}
g \tilde{V}_{3}+g^{2} \phi \tilde{V}_{4} & =g V_{3}+\frac{3}{2} i g^{3} D^{2} V_{3} V_{4}+g^{3} D^{3} V_{3}^{3}+\mathscr{O}\left(g^{5}\right) \\
g^{2} \tilde{V}_{4} & =g^{2} V_{4}+\mathscr{O}\left(g^{4}\right) .
\end{aligned}
$$

Plugging this into (319) and expressing the free, the one-loop and the $\Gamma_{2}$ parts in terms of $V_{3}$ and $V_{4}$ as well as $V_{03}$ and $V_{04}$, one obtains from a straightforward calculation:

$$
\Gamma\left[\phi, D, V_{3}, V_{4}\right]=S[\phi]+\frac{i}{2} \operatorname{Tr} \ln D^{-1}+\frac{i}{2} \operatorname{Tr} D_{0}^{-1}(\phi) D+\Gamma_{2}\left[\phi, D, V_{3}, V_{4}\right],
$$

with

$$
\Gamma_{2}\left[\phi, D, V_{3}, V_{4}\right]=\Gamma_{2}^{0}\left[\phi, D, V_{3}, V_{4}\right]+\Gamma_{2}^{\mathrm{int}}\left[D, V_{3}, V_{4}\right]
$$

\footnotetext{
42 Note that since the exact $\phi$-dependence of $\Gamma_{2}[\phi, D]$ can be written as a function of $\left(g V_{03}+g^{2} \phi V_{04}\right)$, the parametrical dependence of the higher order terms in the variation of (315) with respect to $\left(g V_{03}\right)$ is given by $\mathscr{O}\left(\left.g^{n}\left(g^{2} \phi\right)^{m}\right|_{n+m=5}\right)($ cf. (322)).
} 


$$
\begin{aligned}
\Gamma_{2}^{0}\left[\phi, D, V_{3}, V_{4}\right]= & -\frac{1}{8} g^{2} D^{2} V_{04}+\frac{i}{6} g D^{3} V_{3}\left(g V_{03}+g^{2} \phi V_{04}\right) \\
& +\frac{i}{24} g^{4} D^{4} V_{4} V_{04}+\frac{1}{8} g^{4} D^{5} V_{3}^{2} V_{04}, \\
\Gamma_{2}^{\mathrm{int}}\left[D, V_{3}, V_{4}\right]= & -\frac{i}{12} g^{2} D^{3} V_{3}^{2}-\frac{i}{48} g^{4} D^{4} V_{4}^{2}-\frac{i}{24} g^{4} D^{6} V_{3}^{4}+\mathscr{O}\left(g^{6}\right) .
\end{aligned}
$$

The diagrammatic representation of these results is given in Figs. 19 and 21 of Sec. 6.2.1. There the equivalent calculation is done for a $S U(N)$ gauge theory and one has to replace the propagator lines and vertices of the figures by the corresponding scalar propagator and vertices. Note that for the scalar theory the thick circles represent the dressed three-vertex $g V_{3}$ and four-vertex $g^{2} V_{4}$, respectively, while the small circles denote the corresponding effective classical three-vertex $g V_{03}+g^{2} \phi V_{04}$ and classical four-vertex $g^{2} V_{04}$. As a consequence, the diagrams look the same in the absence of spontaneous symmetry breaking, indicated by a vanishing field expectation value $\phi$.

In (328), the action $S[\phi]$ and $D_{0}$ depend on the classical vertices as before. The expression for $\Gamma_{2}^{0}$, which includes all terms of $\Gamma_{2}$ that depend on the classical vertices, is valid to all orders: $\Gamma_{2}^{\text {int }}$ contains no explicit dependence on the field $\phi$ or the classical vertices $V_{03}$ and $V_{04}$, independent of the approximation for the 4PI effective action. This can be straightforwardly observed from (319), where the complete (linear) dependence of $\Gamma$ on $V_{03}$ and $V_{04}$ is explicit, together with (320) and (321).

\subsubsection{Equivalence hierarchy for nPI effective actions}

As pointed out in Sec. 1.1.2 of the introduction, for applications it is often desirable to obtain a self-consistently complete description, which to a given order of a loop or coupling expansion determines the $n \mathrm{PI}$ effective action $\Gamma\left[\phi, D, V_{3}, V_{4}, \ldots, V_{n}\right]$ for arbitrarily high $n$. Despite the complexity of a general $n \mathrm{PI}$ effective action such a description can be obtained in practice because of the equivalence hierarchy displayed in Eq. (13): Typically the 2PI, 3PI or maybe the 4PI effective action captures already the complete answer for the self-consistent description to the desired/computationally feasible order of approximation. Higher effective actions, which are relevant beyond four-loop order, may not be entirely irrelevant in the presence of sources describing complicated initial conditions for nonequilibrium evolutions. However, their discussion would be somewhat academic from the point of view of calculational feasibility and we will concentrate on up to four-loop corrections or $\mathscr{O}\left(g^{6}\right)$ in the following. Below we will not explicitly write in addition to the loop-order the corresponding order of the coupling $g$ for the considered theory, which is detailed above in Sec. 6.1.1.

To show (13) we will first observe that to one-loop order all $n$ PI effective actions agree in the absence of sources. The one-loop result for the 1PI effective action is given by (18) for vanishing source $R$. As has been explicitly shown in Sec. 2 (cf. Eq. (26)), the one-loop 2PI effective action agrees with that expression, i.e.

$$
\Gamma^{(1 \mathrm{loop})}[\phi, D]=\Gamma^{(1 \mathrm{loop})}[\phi]
$$


in the absence of sources. The equivalence with the one-loop 3PI and 4PI effective actions can be explicitly observed from the results of Sec. 6.1.1. In order to obtain the 3PI expressions we could directly set the source $R_{4} \equiv 0$ from the beginning in the computation of that section such that there is no dependence on $V_{4}$. Equivalently, we can note from Eqs. (328)-(331) that already the 4PI effective action to this order simply agrees with (18) for zero sources. As a consequence, it carries no dependence on $V_{3}$ and $V_{4}$, i.e.

$$
\Gamma^{(1 \text { loop })}\left[\phi, D, V_{3}, V_{4}\right]=\Gamma^{(1 \text { loop })}\left[\phi, D, V_{3}\right]=\Gamma^{(1 \text { loop })}[\phi, D] .
$$

For the one-loop case it remains to be shown that in addition

$$
\Gamma^{(1 \text { loop })}\left[\phi, D, V_{3}, V_{4}, \ldots, V_{n}\right]=\Gamma^{(1 \text { loop })}\left[\phi, D, V_{3}, V_{4}\right]
$$

for arbitrary $n \geq 5$. For this we note that the number $I$ of internal lines in a given loop diagram is given by the number $v_{3}$ of proper 3 -vertices, the number $v_{4}$ of proper 4vertices, $\ldots$, the number $v_{n}$ of proper $n$-vertices in terms of the standard relation:

$$
2 I=3 v_{3}+4 v_{4}+5 v_{5} \ldots+n v_{n}
$$

where $v_{3}+v_{5}+v_{7}+\ldots$ has to be even. Similarly, the number $L$ of loops in such a diagram is

$$
\begin{aligned}
L & =I-v_{3}-v_{4}-v_{5} \ldots-v_{n}+1 \\
& =\frac{1}{2} v_{3}+v_{4}+\frac{3}{2} v_{5} \ldots+\frac{n-2}{2} v_{n}+1 .
\end{aligned}
$$

The equivalence (334) follows from the fact that for $L=1$ equation (336) implies that $\Gamma^{(1 \text { loop })}\left[\phi, D, V_{3}, V_{4}, \ldots, V_{n}\right]$ cannot depend in particular on $V_{5}, \ldots V_{n}{ }^{43}$

The two-loop equivalence of the 2PI and higher effective actions follows along the same lines. According to (328)-(331) the 4PI effective action to two-loop order is given by:

$$
\begin{aligned}
\Gamma^{(2 \text { loop })}\left[\phi, D, V_{3}, V_{4}\right]= & S[\phi]+\frac{i}{2} \operatorname{Tr} \ln D^{-1}+\frac{i}{2} \operatorname{Tr} D_{0}^{-1}(\phi) D \\
& +\Gamma_{2}^{(2 \text { loop })}\left[\phi, D, V_{3}, V_{4}\right] \\
\Gamma_{2}^{(2 \text { loop })}\left[\phi, D, V_{3}, V_{4}\right]= & -\frac{1}{8} g^{2} D^{2} V_{04}+\frac{i}{6} g D^{3} V_{3}\left(g V_{03}+g^{2} \phi V_{04}\right)-\frac{i}{12} g^{2} D^{3} V_{3}^{2} .
\end{aligned}
$$

There is no dependence on $V_{4}$ to this order and, following the discussion above, there is no dependence on $V_{5}, \ldots, V_{n}$ according to (336) for $L=2$. Consequently,

$$
\Gamma^{(2 \mathrm{loop})}\left[\phi, D, V_{3}, V_{4}, \ldots, V_{n}\right]=\Gamma^{(2 \mathrm{loop})}\left[\phi, D, V_{3}, V_{4}\right]=\Gamma^{(2 \mathrm{loop})}\left[\phi, D, V_{3}\right]
$$

\footnotetext{
43 Note that we consider here theories where there is no classical 5-vertex or higher, whose presence would lead to a trivial dependence for the classical action and propagator.
} 
for arbitrary $n$ in the absence of sources. The latter yields

$$
\frac{\delta \Gamma^{(2 \text { loop })}\left[\phi, D, V_{3}\right]}{\delta V_{3}}=\frac{\delta \Gamma_{2}^{(2 \text { loop })}\left[\phi, D, V_{3}\right]}{\delta V_{3}}=0 \Rightarrow g V_{3}=g V_{03}+g^{2} \phi V_{04},
$$

which can be used in (337) to show in addition the equivalence of the 3PI and 2PI effective actions (cf. Eq. (315)) to this order:

$$
\begin{aligned}
\Gamma_{2}^{(2 \text { loop })}\left[\phi, D, V_{3}\right] & =-\frac{1}{8} g^{2} D^{2} V_{04}+\frac{i}{12} D^{3}\left(g V_{03}+g^{2} \phi V_{04}\right)^{2} \\
& =\Gamma_{2}^{(2 \text { loop })}[\phi, D],
\end{aligned}
$$

for vanishing sources. The inequivalence of the 2PI with the 1PI effective action to this order,

$$
\Gamma^{(2 \mathrm{loop})}[\phi, D] \neq \Gamma^{(2 \mathrm{loop})}[\phi],
$$

follows from using the result of $\delta \Gamma_{2}^{(2 l o o p)}[\phi, D] / \delta D=0$ for $D$ in (340) in a straightforward way. ${ }^{44}$

In order to show the three-loop equivalence of the 3PI and higher effective actions, we first note from (328)-(331) that the 4PI effective action to this order yields $V_{4}=V_{04}$ in the absence of sources:

$$
\frac{\delta \Gamma^{(3 \text { loop })}\left[\phi, D, V_{3}, V_{4}\right]}{\delta V_{4}}=\frac{\delta \Gamma_{2}^{(3 \text { loop })}\left[\phi, D, V_{3}, V_{4}\right]}{\delta V_{4}}=\frac{i}{24} g^{4} D^{4}\left(V_{04}-V_{4}\right)=0 .
$$

Constructing the 3PI effective action to three-loop would mean to do the same calculation as in Sec. 6.1.1 but with $V_{4} \rightarrow V_{04}$ from the beginning $\left(R_{4} \equiv 0\right)$. The result of a classical four-vertex for the 4PI effective action to this order, therefore, directly implies:

$$
\Gamma^{(3 \text { loop })}\left[\phi, D, V_{3}, V_{4}\right]=\Gamma^{(3 \text { loop })}\left[\phi, D, V_{3}\right],
$$

for vanishing sources. To see the equivalence with a 5PI effective action $\Gamma^{(3 \text { loop) }}\left[\phi, D, V_{3}, V_{4}, V_{5}\right]$, we note that to three-loop order the only possible diagram including a five-vertex requires $v_{3}=v_{5}=1$ for $L=3$ in Eq. (336). As a consequence, to this order the five-vertex corresponds to the classical one, which is identically zero for the theories considered here, i.e. $V_{5}=V_{05} \equiv 0$. In order to obtain that (to this order trivial) result along the lines of Sec. 6.1.1, one can formally include a classical fivevertex $V_{05}$ and observe that the three-loop $2 \mathrm{PI}$ effective action admits a term $\sim D^{4} V_{05} V_{3}$. After performing the additional Legendre transform the result then follows from setting $V_{05} \rightarrow 0$ in the end. The equivalence with $n$ PI effective actions for $n \geq 6$ can again be observed from the fact that for $L=3$ Eq. (336) implies no dependence on $V_{6}, \ldots V_{n}$. In addition to (343), we therefore have for arbitrary $n \geq 5$ :

$$
\Gamma^{(3 \text { loop })}\left[\phi, D, V_{3}, V_{4}, \ldots, V_{n}\right]=\Gamma^{(3 \text { loop })}\left[\phi, D, V_{3}, V_{4}\right] .
$$

\footnotetext{
${ }^{44}$ Here $\Gamma^{(2 \text { loop })}[\phi, D]$ includes e.g. the summation of an infinite series of so-called "bubble" diagrams, which form the basis of mean-field or Hartree-type approximations, and clearly go beyond a perturbative two-loop approximation $\Gamma^{(2 \mathrm{loop})}[\phi]$ (cf. Sec. 2.1).
} 
The inequivalence of the three-loop 3PI and 2PI effective actions can be readily observed from (328)-(331) and (343):

$$
\frac{\delta \Gamma^{(3 \text { loop })}\left[\phi, D, V_{3}\right]}{\delta V_{3}}=0 \Rightarrow g V_{3}=g\left(V_{03}+g \phi V_{04}\right)-g^{3} D^{3} V_{3}^{3} .
$$

Written iteratively, the above self-consistent equation for $V_{3}$ sums an infinite number of contributions in terms of the classical vertices. As a consequence, the three-loop 3PI result can be written as an infinite series of diagrams for the corresponding 2PI effective action, which clearly goes beyond $\Gamma^{(310 o p)}[\phi, D]$ (cf. Eq. (315)):

$$
\Gamma^{(3 \text { loop })}\left[\phi, D, V_{3}\right] \neq \Gamma^{(3 \text { loop })}[\phi, D] \text {. }
$$

The importance of such an infinite summation will be discussed for the case of gauge theories below.

\subsection{Nonabelian gauge theory with fermions}

We consider a $S U(N)$ gauge theory with $N_{f}$ flavors of Dirac fermions with classical action

$$
\begin{aligned}
S_{\mathrm{eff}} & =S+S_{\mathrm{gf}}+S_{\mathrm{FPG}} \\
& =\int d^{4} x\left(-\frac{1}{4} F_{\mu \nu}^{a} F^{\mu v a}-\frac{1}{2 \xi}\left(\mathscr{G}^{a}(A)\right)^{2}-\bar{\psi}(-i \not D) \psi-\bar{\eta}^{a} \partial_{\mu}\left(D^{\mu} \eta\right)^{a}\right),
\end{aligned}
$$

where $\psi(\bar{\psi}), A$ and $\eta(\bar{\eta})$ denote the (anti-)fermions, gauge and (anti-)ghost fields, respectively, with gauge-fixing term $\mathscr{G}^{a}(A)=\partial^{\mu} A_{\mu}^{a}$ for covariant gauges. The color indices in the adjoint representation are $a, b, \ldots=1, \ldots, N^{2}-1$, while those for the fundamental representation will be denoted by $i, j, \ldots$ and run from 1 to $N$. Here

$$
\begin{aligned}
F_{\mu v}^{a} & =\partial_{\mu} A_{v}^{a}-\partial_{v} A_{\mu}^{a}-g f^{a b c} A_{\mu}^{b} A_{v}^{c} \\
\left(D^{\mu} \eta\right)^{a} & =\partial^{\mu} \eta^{a}-g f^{a b c} A^{\mu b} \eta^{c} \\
\not D & =\gamma^{\mu}\left(\partial_{\mu}+i g A_{\mu}^{a} t^{a}\right)
\end{aligned}
$$

where $\left[t^{a}, t^{b}\right]=i f^{a b c} t^{c}, \operatorname{tr}\left(t^{a} t^{b}\right)=\delta^{a b} / 2$. For QCD, $t^{a}=\lambda^{a} / 2$ with the Gell-Mann matrices $\lambda^{a}(a=1, \ldots, 8)$. We will suppress Dirac and flavor indices in the following. It is convenient to write (347) as

$$
\begin{aligned}
S_{\mathrm{eff}} & =\frac{1}{2} \int_{x y} A^{\mu a}(x) i D_{0 \mu \nu}^{-1 a b}(x, y) A^{v b}(y)+\int_{x y} \bar{\eta}^{a}(x) i G_{0}^{-1 a b}(x, y) \eta^{b}(y) \\
& +\int_{x y} \bar{\psi}_{i}(x) i \Delta_{0 i j}^{-1}(x, y) \psi_{j}(y)-\frac{1}{6} g \int_{x y z} V_{03 \mu v \gamma}^{a b c}(x, y, z) A^{\mu a}(x) A^{v b}(y) A^{\gamma c}(z) \\
& -\frac{1}{24} g^{2} \int_{x y z w} V_{04 \mu v \gamma \delta}^{a b c d}(x, y, z, w) A^{\mu a}(x) A^{v b}(y) A^{\gamma c}(z) A^{\delta d}(w)
\end{aligned}
$$




$$
\begin{aligned}
& -g \int_{x y z} V_{03 \mu}^{(\mathrm{gh}) a b, c}(x, y ; z) \bar{\eta}^{a}(x) \eta^{b}(y) A^{\mu c}(z) \\
& -g \int_{x y z} V_{03 \mu i j}^{(\mathrm{f}) a}(x, y ; z) \bar{\psi}_{i}(x) \psi_{j}(y) A^{\mu a}(z),
\end{aligned}
$$

with the free inverse fermion, ghost and gluon propagator in covariant gauges given by

$$
\begin{aligned}
i \Delta_{0 i j}^{-1}(x, y) & =i \partial_{x} \delta_{i j} \delta_{\mathscr{C}}(x-y), \\
i G_{0}^{-1 a b}(x, y) & =-\square_{x} \delta^{a b} \delta_{\mathscr{C}}(x-y), \\
i D_{0 \mu \nu}^{-1 a b}(x, y) & =\left[g_{\mu \nu} \square-\left(1-\xi^{-1}\right) \partial_{\mu} \partial_{v}\right]_{x} \delta^{a b} \delta_{\mathscr{C}}(x-y),
\end{aligned}
$$

where we have taken the fermions to be massless. The tree-level vertices read in coordinate space:

$$
\begin{aligned}
V_{03 \mu \nu \gamma}^{a b c}(x, y, z)= & f^{a b c}( \\
& g_{\mu v}\left[\delta_{\mathscr{C}}(y-z) \partial_{\gamma}^{x} \delta_{\mathscr{C}}(x-y)-\delta_{\mathscr{C}}(x-z) \partial_{\gamma}^{y} \delta_{\mathscr{C}}(y-x)\right] \\
+ & g_{\mu \gamma}\left[\delta_{\mathscr{C}}(x-y) \partial_{\nu}^{z} \delta_{\mathscr{C}}(z-x)-\delta_{\mathscr{C}}(y-z) \partial_{\nu}^{x} \delta_{\mathscr{C}}(x-z)\right] \\
+ & \left.g_{v \gamma}\left[\delta_{\mathscr{C}}(x-z) \partial_{\mu}^{y} \delta_{\mathscr{C}}(y-x)-\delta_{\mathscr{C}}(x-y) \partial_{\mu}^{z} \delta_{\mathscr{C}}(z-x)\right]\right) \\
V_{04 \mu v \gamma \delta}^{a b c d}(x, y, z, w)= & \left(f^{a b e} f^{c d e}\left[g_{\mu \gamma} g_{v \delta}-g_{\mu \delta} g_{v \gamma}\right]\right. \\
+ & \left.f^{a c e} f^{b d e}\left[g_{\mu v} g_{\gamma \delta}-g_{\mu \delta} g_{v \gamma}\right]+f^{a d e} f^{c b e}\left[g_{\mu \gamma} g_{\delta v}-g_{\mu \nu} g_{\gamma \delta}\right]\right) \\
& \delta_{\mathscr{C}}(x-y) \delta_{\mathscr{C}}(x-z) \delta_{\mathscr{C}}(x-w) \\
V_{03 \mu}^{(\mathrm{gh}) a b, c}(x, y ; z)= & -f^{a b c} \partial_{\mu}^{x} \delta_{\mathscr{C}}(x-z) \delta_{\mathscr{C}}(y-z) \\
V_{03 \mu i j}^{(\mathrm{f}) a}(x, y ; z)= & \gamma_{\mu} t_{i j}^{a} \delta_{\mathscr{C}}(x-z) \delta_{\mathscr{C}}(z-y) .
\end{aligned}
$$

Note that $V_{03, a b c}^{\mu v \gamma}(x, y, z)$ is symmetric under exchange of $(\mu, a, x) \leftrightarrow(\nu, b, y) \leftrightarrow(\gamma, c, z)$. Likewise, $V_{04, a b c d}^{\mu v \gamma \delta}(x, y, z, w)$ is symmetric in its space-time arguments and under exchange of $(\mu, a) \leftrightarrow(v, b) \leftrightarrow(\gamma, c) \leftrightarrow(\delta, d)$.

In addition to the linear and bilinear source terms, which are required for a construction of the 2PI effective action, following Sec. 6.1 we add cubic and quartic source terms to $(351)$ :

$$
\begin{aligned}
S_{\text {source }}^{\prime} & =\frac{1}{6} \int_{x y z} R_{3 \mu v \gamma}^{a b c}(x, y, z) A^{\mu a}(x) A^{v b}(y) A^{\gamma c}(z) \\
& +\frac{1}{24} \int_{x y z w} R_{4 \mu v \gamma \delta}^{a b c d}(x, y, z, w) A^{\mu a}(x) A^{v b}(y) A^{\gamma c}(z) A^{\delta d}(w) \\
& +\int_{x y z} R_{3 \mu}^{(\mathrm{gh}) a b, c}(x, y ; z) \bar{\eta}^{a}(x) \eta^{b}(y) A^{\mu c}(z)
\end{aligned}
$$




$$
+\int_{x y z} R_{3 \mu i j}^{(\mathrm{f}) a}(x, y ; z) \bar{\psi}_{i}(x) \psi_{j}(y) A^{\mu a}(z)
$$

where the sources $R_{3,4}$ obey the same symmetry properties as the corresponding classical vertices $V_{03}$ and $V_{04}$ discussed above. The definition of the corresponding three- and fourvertices follows Sec. 6.1. In particular, we have for the vertices involving Grassmann fields:

$$
\begin{aligned}
\frac{\delta W}{\delta R_{3 \mu}^{(\mathrm{gh}) a b, c}(x, y ; z)} & =-i g \int_{x^{\prime} y^{\prime} z^{\prime}} D^{\mu \mu^{\prime} c c^{\prime}}\left(z, z^{\prime}\right) G^{b a^{\prime}}\left(y, x^{\prime}\right) V_{3 \mu^{\prime}}^{(\mathrm{gh}) a^{\prime} b^{\prime} c^{\prime}}\left(x^{\prime}, y^{\prime} ; z^{\prime}\right) G^{b^{\prime} a}\left(y^{\prime}, x\right), \\
\frac{\delta W}{\delta R_{3 \mu i j}^{(\mathrm{f}) a}(x, y ; z)} & =-i g \int_{x^{\prime} y^{\prime} z^{\prime}} D^{\mu \mu^{\prime} a a^{\prime}}\left(z, z^{\prime}\right) \Delta_{j i^{\prime}}\left(y, x^{\prime}\right) V_{3 \mu^{\prime} i^{\prime} j^{\prime}}^{(\mathrm{f}) a^{\prime}}\left(x^{\prime}, y^{\prime} ; z^{\prime}\right) \Delta_{j^{\prime} i}\left(y^{\prime}, x\right),(360)
\end{aligned}
$$

for the case of vanishing "background" fields $\langle A\rangle=\langle\psi\rangle=\langle\bar{\psi}\rangle=\langle\eta\rangle=\langle\bar{\eta}\rangle=0$, which we will consider in the following.

\subsubsection{Effective action up to four-loop or $\mathscr{O}\left(g^{6}\right)$ corrections}

Consider first the 2PI effective action with vanishing "background" fields, which according to Sec. 2 can be written as

$$
\begin{aligned}
\Gamma[D, \Delta, G]= & \frac{i}{2} \operatorname{Tr} \ln D^{-1}+\frac{i}{2} \operatorname{Tr} D_{0}^{-1} D-i \operatorname{Tr} \ln \Delta^{-1}-i \operatorname{Tr} \Delta_{0}^{-1} \Delta \\
& -i \operatorname{Tr} \ln G^{-1}-i \operatorname{Tr} G_{0}^{-1} G+\Gamma_{2}[D, \Delta, G] .
\end{aligned}
$$

Here the trace $\operatorname{Tr}$ includes an integration over the time path $\mathscr{C}$, as well as integration over spatial coordinates and summation over flavor, color and Dirac indices. The exact expression for $\Gamma_{2}$ contains all 2PI diagrams with vertices described by (355)-(358) and propagator lines associated to the full connected two-point functions $D, G$ and $\Delta$. In order to clear up the presentation, we will give all diagrams including gauge and ghost propagators only. The fermion diagrams can simply be obtained from the corresponding ghost ones, since they have the same signs and prefactors. ${ }^{45}$ For the $2 \mathrm{PI}$ effective action of the gluon-ghost system, $\Gamma[D, G]$, to three-loop order the 2PI effective action is given by (using the same compact notation as introduced in Sec. 6.1.1):

$$
\begin{aligned}
\Gamma_{2}[D, G]= & -\frac{1}{8} g^{2} D^{2} V_{04}+\frac{i}{12} g^{2} D^{3} V_{03}^{2}-\frac{i}{2} g^{2} D G^{2} V_{03}^{(\mathrm{gh}) 2}+\frac{i}{48} g^{4} D^{4} V_{04}^{2} \\
& +\frac{1}{8} g^{4} D^{5} V_{03}^{2} V_{04}-\frac{i}{24} g^{4} D^{6} V_{03}^{4}+\frac{i}{3} g^{4} D^{3} G^{3} V_{03}^{(\mathrm{gh}) 3} V_{03} \\
& +\frac{i}{4} g^{4} D^{2} G^{4} V_{03}^{(\mathrm{gh}) 4}+\mathscr{O}\left(g^{6}\right) .
\end{aligned}
$$

\footnotetext{
45 Note that to three-loop order there are no graphs with more than one closed ghost/fermion loop, such that ghosts and fermions cannot appear in the same diagram simultaneously.
} 


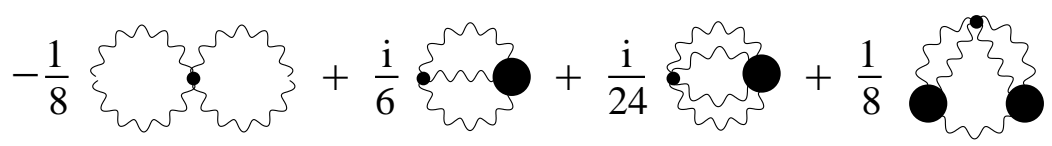

FIGURE 19. The figure shows together with Fig. 20 the diagrammatic representation of $\Gamma_{2}^{0}\left[D, G, V_{3}, V_{3}^{(\mathrm{gh})}, V_{4}\right]$ as given in Eq. (365). Here the wiggled lines denote the gauge field propagator $D$ and the unwiggled lines the ghost propagator $G$. The thick circles denote the dressed and the small ones the classical vertices. This functional contains all terms of $\Gamma_{2}$ that depend on the classical vertices $g V_{03}$, $g V_{03}^{(\mathrm{gh})}$ and $g^{2} V_{04}$ for an $S U(N)$ gauge theory. There are no further contributions to $\Gamma_{2}^{0}$ appearing at higher order in the expansion. For the gauge theory with fermions there is in addition the same contribution as in Fig. 20 with the unwiggled propagator lines representing the fermion propagator $\Delta$ and the ghost vertices replaced by the corresponding fermion vertices $V_{03}^{(\mathrm{f})}$ and $V_{3}^{(\mathrm{f})}$ (cf. Eq. (358)).

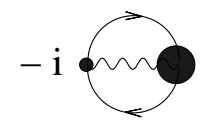

FIGURE 20. Ghost/fermion part of $\Gamma_{2}^{0}$.

The result can be compared with (315) and taking into account an additional factor of $(-1)$ for each closed loop involving Grassmann fields (cf. Sec. 2.3). Here we have suppressed in the notation the dependence of $\Gamma_{2}[D, G]$ on the higher sources (359). The desired effective action is obtained by performing the remaining Legendre transforms:

$$
\Gamma\left[D, G, V_{3}, V_{3}^{(\mathrm{gh})}, V_{4}\right]=\Gamma[D, G]-\frac{\delta W}{\delta R_{3}} R_{3}-\frac{\delta W}{\delta R_{3}^{(\mathrm{gh})}} R_{3}^{(\mathrm{gh})}-\frac{\delta W}{\delta R_{4}} R_{4} .
$$

The calculation follows the same steps as detailed in Sec. 6.1.1. For the effective action to $\mathscr{O}\left(g^{6}\right)$ we obtain:

$$
\begin{aligned}
\Gamma\left[D, G, V_{3}, V_{3}^{(\mathrm{gh})}, V_{4}\right]= & \frac{i}{2} \operatorname{Tr} \ln D^{-1}+\frac{i}{2} \operatorname{Tr} D_{0}^{-1} D-i \operatorname{Tr} \ln G^{-1}-i \operatorname{Tr} G_{0}^{-1} G \\
& +\Gamma_{2}\left[D, G, V_{3}, V_{3}^{(\mathrm{gh})}, V_{4}\right]
\end{aligned}
$$

with

$$
\begin{aligned}
\Gamma_{2}\left[D, G, V_{3}, V_{3}^{(\mathrm{gh})}, V_{4}\right]= & \Gamma_{2}^{0}\left[D, G, V_{3}, V_{3}^{(\mathrm{gh})}, V_{4}\right]+\Gamma_{2}^{\mathrm{int}}\left[D, G, V_{3}, V_{3}^{(\mathrm{gh})}, V_{4}\right] \\
\Gamma_{2}^{0}\left[D, G, V_{3}, V_{3}^{(\mathrm{gh})}, V_{4}\right]= & -\frac{1}{8} g^{2} D^{2} V_{04}+\frac{i}{6} g^{2} D^{3} V_{3} V_{03}-i g^{2} D G^{2} V_{3}^{(\mathrm{gh})} V_{03}^{(\mathrm{gh})} \\
& +\frac{i}{24} g^{4} D^{4} V_{4} V_{04}+\frac{1}{8} g^{4} D^{5} V_{3}^{2} V_{04}, \\
\Gamma_{2}^{\text {int }}\left[D, G, V_{3}, V_{3}^{(\mathrm{gh})}, V_{4}\right]= & -\frac{i}{12} g^{2} D^{3} V_{3}^{2}+\frac{i}{2} g^{2} D G^{2} V_{3}^{(\mathrm{gh}) 2}-\frac{i}{48} g^{4} D^{4} V_{4}^{2} \\
& -\frac{i}{24} g^{4} D^{6} V_{3}^{4}+\frac{i}{3} g^{4} D^{3} G^{3} V_{3}^{(\mathrm{gh}) 3} V_{3} \\
& +\frac{i}{4} g^{4} D^{2} G^{4} V_{3}^{(\mathrm{gh}) 4}+\mathscr{O}\left(g^{6}\right) .
\end{aligned}
$$




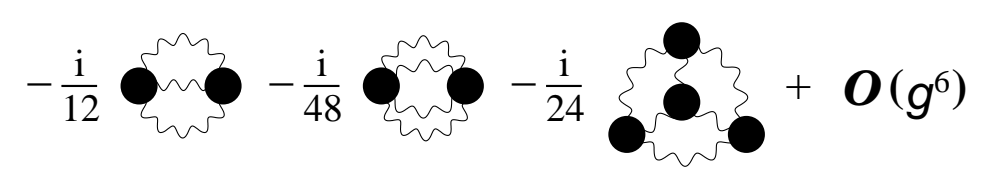

FIGURE 21. The figure shows together with Fig. 22 the diagrammatic representation of $\Gamma_{2}^{\text {int }}\left[D, G, V_{3}, V_{3}^{(\mathrm{gh})}, V_{4}\right]$ to three-loop order as given in Eq. (366). For the gauge theory with fermions, to this order there is in addition the same contribution as in Fig. 22 with the unwiggled propagator lines representing the fermion propagator $\Delta$ and the ghost vertex replaced by the corresponding fermion vertex $V_{3}^{(\mathrm{f})}$. This functional contains no explicit dependence on the classical vertices independent of the order of approximation.

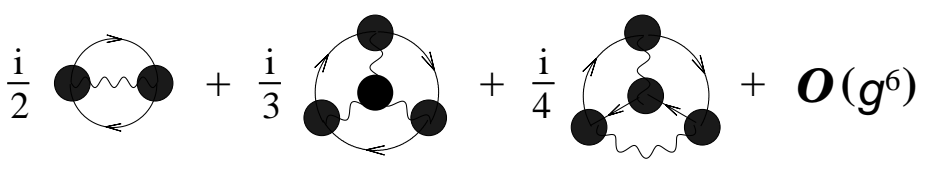

FIGURE 22. Ghost/fermion part of $\Gamma_{2}^{\text {int }}$ to three-loop order.

The contributions are displayed diagrammatically in Figs. 19 and 20 for $\Gamma_{2}^{0}$, and in Figs. 21 and 22 for $\Gamma_{2}^{\text {int }}$.

The equivalence of the 4PI effective action to three-loop order with the 3PI and $n \mathrm{PI}$ effective actions for $n \geq 5$ in the absence of sources follows along the lines of Sec. 6.1.2. As a consequence, to three-loop order the $n$ PI effective action does not depend on higher vertices $V_{5}, V_{6}, \ldots V_{n}$. In particular with vanishing sources the four-vertex is given by the classical one:

$$
\frac{\delta \Gamma^{(3 l o o p)}\left[D, G, V_{3}, V_{3}^{(\mathrm{gh})}, V_{4}\right]}{\delta V_{4}}=\frac{\delta \Gamma_{2}^{(3 l o o p)}\left[D, G, V_{3}, V_{3}^{(\mathrm{gh})}, V_{4}\right]}{\delta V_{4}}=0 \Rightarrow V_{4}=V_{04}
$$

If one plugs this into (365) and (366) one obtains the three-loop 3PI effective action, $\Gamma^{(3 \text { loop })}\left[\phi, D, V_{3}, V_{3}^{(\mathrm{gh})}\right]$. Similarly, to two-loop order one has

$$
\begin{aligned}
& \frac{\delta \Gamma^{(2 \text { loop })}\left[D, G, V_{3}, V_{3}^{(\mathrm{gh})}\right]}{\delta V_{3}}=\frac{\delta \Gamma_{2}^{(2 \mathrm{loop})}\left[D, G, V_{3}, V_{3}^{(\mathrm{gh})}\right]}{\delta V_{3}}=0 \Rightarrow V_{3}=V_{03}, \\
& \frac{\delta \Gamma^{(2 \mathrm{loop})}\left[D, G, V_{3}, V_{3}^{(\mathrm{gh})}\right]}{\delta V_{3}^{(\mathrm{gh})}}=\frac{\delta \Gamma_{2}^{(2 \mathrm{loop})}\left[D, G, V_{3}, V_{3}^{(\mathrm{gh})}\right]}{\delta V_{3}^{(\mathrm{gh})}}=0 \Rightarrow V_{3}^{(\mathrm{gh})}=V_{03}^{(\mathrm{gh})},
\end{aligned}
$$

and equivalently for the fermion vertex $V_{3}^{(\mathrm{f})}$. To this order, therefore, the combinatorial factors of the two-loop diagrams of Fig. 19 and 21 for the gauge part, as well as of Fig. 20 and 22 for the ghost/fermion part, combine to give the result (362) to two-loop order for the 2PI effective action.

We have seen above that to two-loop order the proper vertices of the $n$ PI effective action correspond to the classical ones. Accordingly, at this order the only non-trivial equations of motion in the absence of "background" fields are those for the two-point 


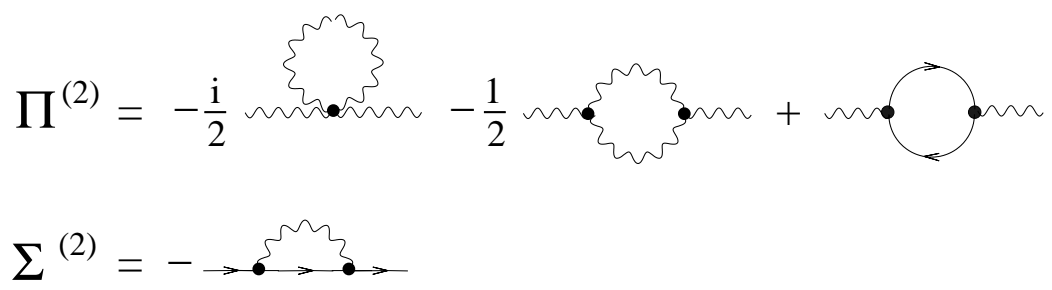

FIGURE 23. The self-energy for the gauge field $(\Pi)$ and the ghost/fermion $(\Sigma)$ propagators as obtained from the self-consistently complete two-loop approximation of the effective action. Note that at this order all vertices correspond to the classical ones.

functions:

$$
\frac{\delta \Gamma}{\delta D}=0, \quad \frac{\delta \Gamma}{\delta G}=0, \quad \frac{\delta \Gamma}{\delta \Delta}=0
$$

for vanishing sources. Applied to an $n$ PI effective action $(n>1)$, as e.g. (364), one finds for the gauge field propagator:

$$
D^{-1}=D_{0}^{-1}-\Pi \quad, \quad \Pi=2 i \frac{\delta \Gamma_{2}}{\delta D}
$$

The ghost propagator and self-energy are

$$
G^{-1}=G_{0}^{-1}-\Sigma \quad, \quad \Sigma=-i \frac{\delta \Gamma_{2}}{\delta G},
$$

and equivalently for the fermion propagator $\Delta$. (Cf. also Sec. 2 for the same relations in the context of 2PI effective actions.) The self-energies to this order are shown in diagrammatic form in Fig. 23. In contrast, for the three-loop effective action the threevertices get dressed and the stationarity conditions,

$$
\frac{\delta \Gamma}{\delta V_{3}}=0, \quad \frac{\delta \Gamma}{\delta V_{3}^{(\mathrm{gh})}}=0, \quad \frac{\delta \Gamma}{\delta V_{3}^{(\mathrm{f})}}=0,
$$

applied to (364)-(366) lead to the equations shown in the left graph of Fig. 25. Here the diagrammatic form of the contributions is always the same for the ghost and for the fermion propagators or vertices. We therefore only give the expressions for the gaugeghost system. If fermions are present, the respective diagrams have to be added in a straightforward way.

The self-energies to this order are displayed in Fig. 24. It should be emphasized that their relatively simple form is a consequence of the equations for the proper vertices, Fig. 25. To see this we consider first the many terms generated by the functional derivative of (365) and (366) with respect to the gauge field propagator:

$$
\begin{aligned}
& \Pi^{(3)} \equiv 2 i \frac{\delta \Gamma_{2}^{(3 l o o p)}}{\delta D}=-\frac{i}{2} \sum^{n} \xi^{2}-\frac{1}{2}
\end{aligned}
$$

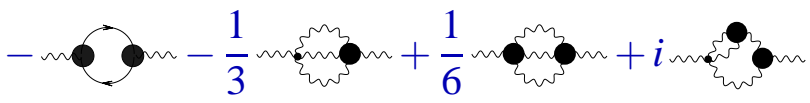

$$
\begin{aligned}
& +\frac{i}{4} m+\frac{1}{2} m \xi-2 m-
\end{aligned}
$$




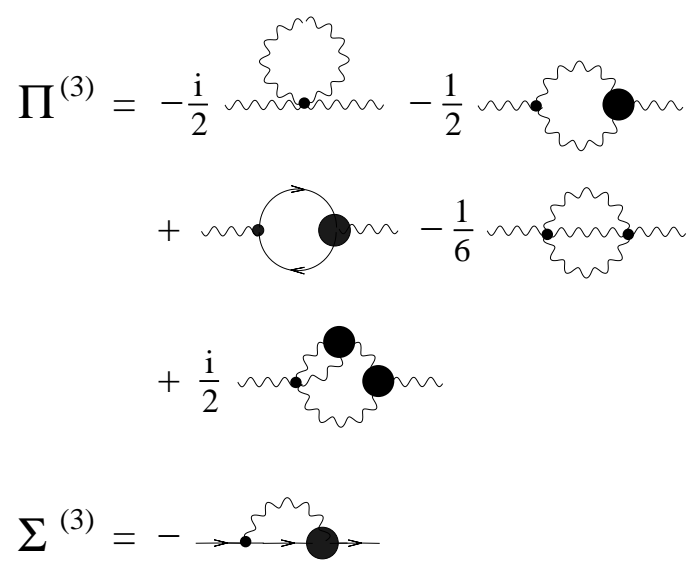

FIGURE 24. The self-energy for the gauge field $(\Pi)$ and the ghost/fermion $(\Sigma)$ propagators as obtained from the self-consistently complete three-loop approximation of the effective action. (Cf. Fig. 25 for the vertices.)

The short form for the self-energy of Fig. 24 is obtained through cancellations by replacing in the above expression

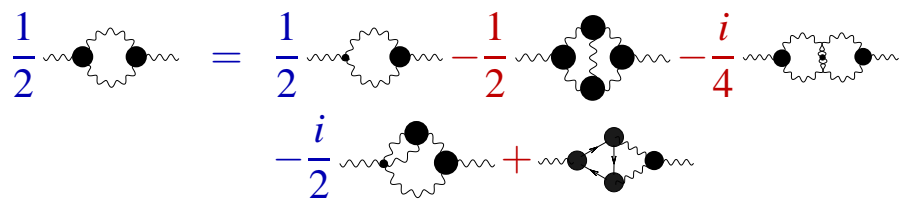

as well as

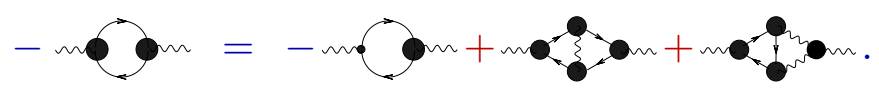

The latter equations follow from inserting the expressions for the dressed vertices of Fig. 25. Noting in addition that the proper four-vertex to this order corresponds to the classical one (cf. (367)) leads to the result. Along the very same lines a similar cancellation yields the compact form of the ghost/fermion self-energy displayed in Fig. 24.

\subsubsection{Comparison with Schwinger-Dyson equations}

The equations of motions of the last section are self-consistently complete to twoloop/three-loop order of the $n$ PI effective action for arbitrarily large $n$. We now compare them with Schwinger-Dyson (SD) equations, which represent identities between $n$-point functions. Clearly, without approximations the equations of motion obtained from an exact $n$ PI effective action and the exact (SD) equations have to agree since one can always map identities onto each other. However, in general this is no longer the case for a given order in the loop expansion of the $n \mathrm{PI}$ effective action.

By construction each diagram in a SD equation contains at least one classical vertex. In general, this is not the case for equations obtained from the $n$ PI effective action: The 


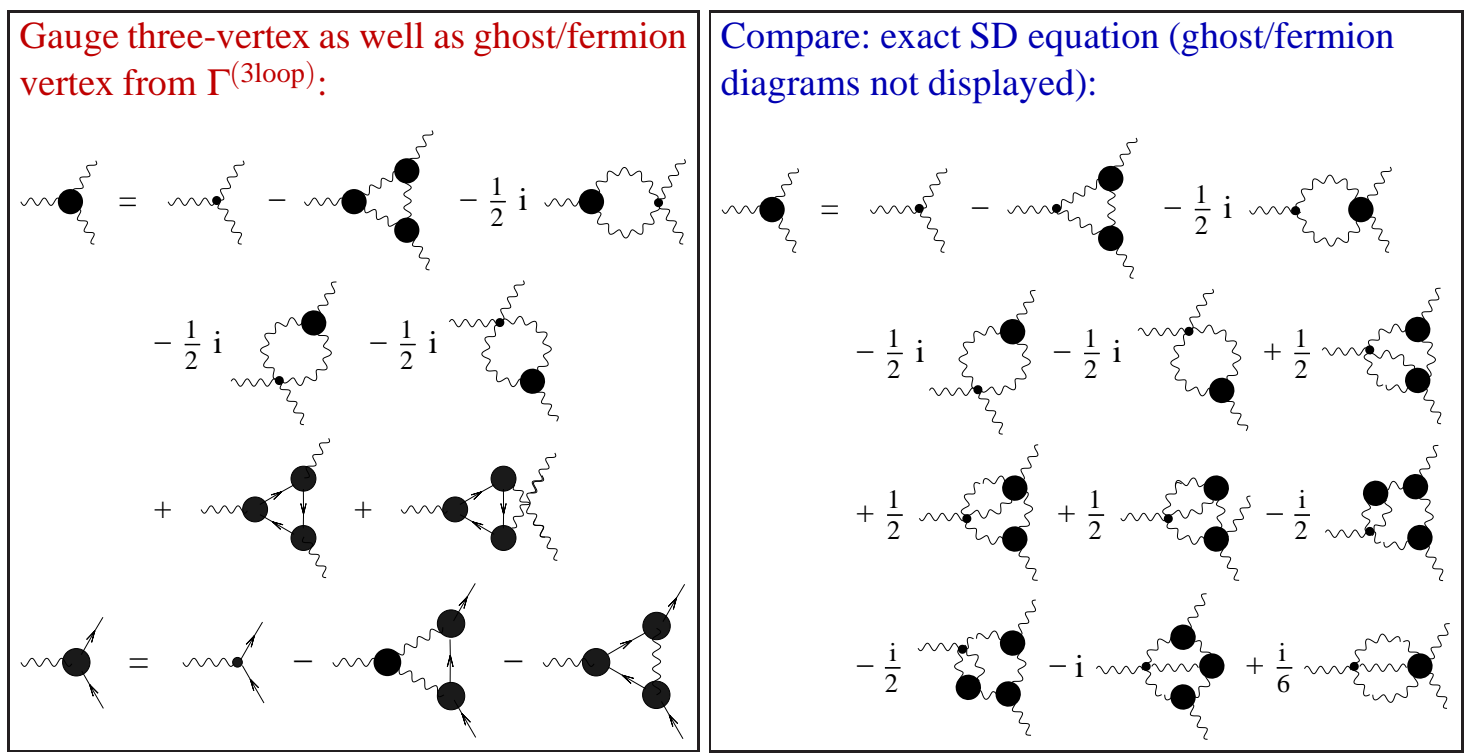

FIGURE 25. Left: The gauge field three-vertex as well as the ghost (fermion) vertex as obtained from the self-consistently complete three-loop approximation of the effective action. Apart from the isolated classical three-vertex, all vertices in the equations correspond to dressed ones since at this order the fourvertex equals the classical vertex. The result reflects the proper symmetry of the three-vertex. Right: Schwinger-Dyson equation for the proper three-vertex $V_{3}$. Additional diagrams involving ghost or fermion vertices are not displayed for brevity. We show it for comparison with the three-loop effective action result displayed on the left. A naive truncation of the Schwinger-Dyson equation at the one-loop level does not agree with the latter and symmetries (the second and third diagram contain a classical three-vertex instead of a dressed one).

loop contributions of $\Gamma_{2}^{\text {int }}$ in Eq. (366) or Figs. 21-22 are solely expressed in terms of full vertices. However, to a given loop-order cancellations can occur for those diagrams in the equations of motion which do not contain a classical vertex. For the three-loop effective action result this has been demonstrated above for the two-point functions. Indeed, the equations for the two-point functions shown in Fig. 24 correspond to the SD equations, if one takes into account that to the considered order the four-vertex is trivial and given by the classical one (cf. Eq. (367)). However, such a correspondence is not true for the proper three-vertex to that order.

As an example, we show on the right of Fig. 25 the SD equation for the proper three-vertex, where for brevity we do not display the additional diagrams coming from ghost/fermion degrees of freedom. One observes that a naive neglection of the two-loop contributions of that equation would not lead to the effective action result for the threevertex shown in Fig. 25. Of course, the straightforward one-loop truncation of the SD equation would not even respect the property of $V_{3}$ being completely symmetric in its space-time and group labels. This is the well-known problem of loop-expansions of SD equations, where one encounters the ambiguity of whether classical or dressed vertices should be employed at a given truncation order.

We emphasize that these problems are absent using effective action techniques. The fact that all equations of motion are obtained from the same approximation of the effective action puts stringent conditions on their form. More precisely, a self-consistently 
complete approximation has the property that the order of differentiation of, say, $\Gamma[D, V]$ with respect to the propagator $D$ or the vertex $V$ does not affect the equations of motion. Consider for instance:

$$
\frac{\delta \Gamma[D, V=V(D)]}{\delta D}=\left.\frac{\delta \Gamma}{\delta D}\right|_{V}+\left.\frac{\delta \Gamma}{\delta V}\right|_{D} \frac{\delta V}{\delta D} .
$$

If $V=V(D)$ is the result of the stationary condition $\delta \Gamma / \delta V=0$ then the above corresponds to the correct stationarity condition for the propagator for fixed $V: \delta \Gamma / \delta D=0$. In contrast, with some ansatz $V=f(D)$ that does not fulfill the stationarity condition of the effective action, the equation of motion for the propagator would receive additional corrections $\sim \delta V / \delta D$. In particular, it would be inconsistent to use the equation of motion for the propagator $\delta \Gamma / \delta D=0$ (cf. e.g. Fig. 24 which corresponds to the SD equation result) but not the equation $\delta \Gamma / \delta V=0$ for the vertex (cf. Fig. 25).

In turn, one can conclude that a wide class of employed truncations of exact SD equations cannot be obtained from the $n$ PI effective action: this concerns those approximations which use the exact SD equation for the propagator but make an ansatz for the vertices that differs from the one displayed in Fig. 25. The differences are, however, typically higher order in the perturbative coupling expansion and there may be many cases, in particular in vacuum or thermal equilibrium, where some ansatz for the vertices is a very efficient way to proceed. Out of equilibrium however, as mentioned above, the "conserving" property of the effective action approximations can have important consequences, since the effective loss of initial conditions and the presence of basic constants of motion such as energy conservation is crucial.

\subsubsection{Nonequilibrium evolution equations}

Up to $\mathscr{O}\left(g^{6}\right)$ corrections in the self-consistently complete expansion of the effective action, the four-vertex parametrizing the diagrams of Figs. 24-25 corresponds to the classical vertex. At this order of approximation there is, therefore, no distinction between the coupling expansion of the 3PI and 4PI effective action. To discuss the relevant differences between the 2PI and 3PI expansions for time evolution problems, we will use the language of QED for simplicity, where no four-vertex appears. However, the evolution equations of this section can be straightforwardly transcribed to the nonabelian case by taking into account in addition to the equation for the gauge-fermion threevertex those for the gauge-ghost and gauge three-vertex (cf. Fig. 25). In the following the effective action is a functional of the gauge field propagator $D_{\mu v}(x, y)$, the fermion propagator $\Delta(x, y)$ and the gauge-fermion vertex $V_{3 \mu}^{(\mathrm{f})}(x, y ; z)$, where we suppress Dirac indices and we will write $V_{3}^{(\mathrm{f})} \equiv V$. According to Eqs. (364)-(366) one has in this case

$$
\Gamma_{2}[D, \Delta, V]=\Gamma_{2}^{0}[D, \Delta, V]+\Gamma_{2}^{\text {int }}[D, \Delta, V]
$$

with

$$
\Gamma_{2}^{0}=-i g^{2} \int_{x y z u} \operatorname{tr}\left[\gamma_{\mu} \Delta(x, y) V_{v}(y, z ; u) \Delta(z, x) D^{\mu v}(x, u)\right]
$$


where the trace acts in Dirac space. For the given order of approximation there are two distinct contributions to $\Gamma_{2}^{\text {int }}$ :

$$
\begin{aligned}
\Gamma_{2}^{\mathrm{int}}= & \Gamma_{2}^{(a)}+\Gamma_{2}^{(b)}+\mathscr{O}\left(g^{6}\right), \\
\Gamma_{2}^{(a)}= & \frac{i}{2} g^{2} \int_{x y z u v w} \operatorname{tr}\left[V_{\mu}(x, y ; z) \Delta(y, u) V_{v}(u, v ; w) \Delta(v, x) D^{\mu v}(z, w)\right], \\
\Gamma_{2}^{(b)}= & \frac{i}{4} g^{4} \int_{x y z u v w x^{\prime} y^{\prime} z^{\prime} u^{\prime} v^{\prime} w^{\prime}} \operatorname{tr}\left[V_{\mu}(x, y ; z) \Delta(y, u) V_{v}(u, v ; w) \Delta\left(v, x^{\prime}\right)\right. \\
& \left.V_{\rho}\left(x^{\prime}, y^{\prime} ; z^{\prime}\right) \Delta\left(y^{\prime}, u^{\prime}\right) V_{\sigma}\left(u^{\prime}, v^{\prime} ; w^{\prime}\right) \Delta\left(v^{\prime}, x\right) D^{\mu \rho}\left(z, z^{\prime}\right) D^{v \sigma}\left(w, w^{\prime}\right)\right] .
\end{aligned}
$$

The equations of motions for the propagators and vertex are obtained from the stationarity conditions (368) and (371) for the effective action. To convert (369) for the photon propagator into an equation which is more suitable for initial value problems, we convolute with $D$ from the right and obtain for the considered case of vanishing "background" fields, e.g. for covariant gauges (cf. also the discussion in Sec. 3.4):

$$
\begin{array}{r}
{\left[g_{\gamma}^{\mu} \square-\left(1-\xi^{-1}\right) \partial^{\mu} \partial_{\gamma}\right]_{x} D^{\gamma v}(x, y)-i \int_{z} \Pi_{\gamma}^{\mu}(x, z) D^{\gamma v}(z, y)} \\
=i g^{\mu v} \delta_{\mathscr{C}}(x-y)
\end{array}
$$

Similarly, the corresponding equation of (370) yields the evolution equation for the fermion propagator given in Eq. (141). Using the above results the self-energies are

$$
\begin{aligned}
& \Sigma^{(f)}(x, y)=-g^{2} \int_{z^{\prime} z^{\prime \prime}} D_{\mu v}\left(z^{\prime}, y\right) V^{\mu}\left(x, z^{\prime \prime} ; z^{\prime}\right) \Delta\left(z^{\prime \prime}, y\right) \gamma^{v} \\
& \Pi^{\mu v}(x, y)=g^{2} \int_{z^{\prime} z^{\prime \prime}} \operatorname{tr} \gamma^{\mu} \Delta\left(x, z^{\prime}\right) V^{v}\left(z^{\prime}, z^{\prime \prime} ; y\right) \Delta\left(z^{\prime \prime}, x\right) .
\end{aligned}
$$

Note that the form of the self-energies is exact for known three-vertex. To see this within the current framework, we note that the self-energies can be expressed in terms of $\Gamma_{2}^{0}$ only. The latter receives no further corrections at higher order in the expansion (cf. Sec. 6.2.1), and thus the expression is exactly known: With

$$
\int_{z} \Sigma^{(f)}(x, z) \Delta(z, y)=-i \int_{z}\left(\frac{\delta \Gamma_{2}^{0}}{\delta \Delta(z, x)}+\frac{\delta \Gamma_{2}^{\mathrm{int}}}{\delta \Delta(z, x)}\right) \Delta(z, y),
$$

and since $\Gamma_{2}^{\text {int }}$ is only a functional of $V \Delta D^{1 / 2}$ (cf. Sec. 6.2.1) one can use the identity

$$
\begin{aligned}
\int_{z} \frac{\delta \Gamma_{2}^{\text {int }}}{\delta \Delta(z, x)} \Delta(z, y) & =\int_{z z^{\prime}} V_{\mu}\left(x, z ; z^{\prime}\right) \frac{\delta \Gamma_{2}^{\text {int }}}{V_{\mu}\left(y, z ; z^{\prime}\right)} \\
& =-\int_{z z^{\prime}} V_{\mu}\left(x, z ; z^{\prime}\right) \frac{\delta \Gamma_{2}^{0}}{V_{\mu}\left(y, z ; z^{\prime}\right)}
\end{aligned}
$$


to express everything in terms of the known ${ }^{46} \Gamma_{2}^{0}$. The last equality in (383) uses that $\delta\left(\Gamma_{2}^{0}+\Gamma_{2}^{\text {int }}\right) / \delta \Delta=0$. A similar discussion can be done for the photon self-energy. As a consequence, all approximations are encoded in the equation for the vertex, which is obtained from (378) as

$$
\begin{aligned}
V^{\mu}(x, y ; z)= & V_{0}^{\mu}(x, y ; z)-g^{2} \int_{v w x^{\prime} y^{\prime} u^{\prime} w^{\prime}} V_{v}(x, v ; w) \Delta\left(v, x^{\prime}\right) V^{\mu}\left(x^{\prime}, y^{\prime} ; z\right) \\
& \Delta\left(y^{\prime}, u^{\prime}\right) V_{\sigma}\left(u^{\prime}, y ; w^{\prime}\right) D^{\sigma v}\left(w^{\prime}, w\right)+\mathscr{O}\left(g^{4}\right),
\end{aligned}
$$

where

$$
V_{0}^{\mu}(x, y ; z)=\gamma^{\mu} \delta(x-z) \delta(z-y) .
$$

For the self-consistently complete two-loop approximation the self-energies are given by

$$
\begin{aligned}
& \Sigma^{(f)}(x, y)=-g^{2} D_{\mu v}(x, y) \gamma^{\mu} \Delta(x, y) \gamma^{v}+\mathscr{O}\left(g^{4}\right) \\
& \Pi^{\mu v}(x, y)=g^{2} \operatorname{tr} \gamma^{\mu} \Delta(x, y) \gamma^{v} \Delta(y, x)+\mathscr{O}\left(g^{4}\right) .
\end{aligned}
$$

Following the discussion of Sec. 3.4.1, we decompose the two-point functions into spectral and statistical components using the identities (143) for gauge fields and (121) for fermions. Then $\rho_{D}$ corresponds to the gauge field spectral function and $F_{D}$ is the statistical two-point function, while $\rho^{(f)}$ and $F^{(f)}$ are the corresponding fermion two-point functions. The same decomposition can be done for the corresponding self-energies:

$$
\begin{aligned}
\Pi^{\mu v}(x, y) & =\Pi_{(F)}^{\mu v}(x, y)-\frac{i}{2} \Pi_{(\rho)}^{\mu v}(x, y) \operatorname{sign}\left(x^{0}-y^{0}\right), \\
\Sigma^{(f)}(x, y) & =\Sigma_{F}^{(f)}(x, y)-\frac{i}{2} \Sigma_{\rho}^{(f)}(x, y) \operatorname{sign}\left(x^{0}-y^{0}\right),
\end{aligned}
$$

and similarly for the fermions as described by (126). Since the above decomposition for the propagators and self-energies makes the time-ordering explicit, we can evaluate the r.h.s. of (379) along the time contour following the discussion of Sec. 3.4.3. One finds the evolution equations:

$$
\begin{aligned}
{\left[g^{\mu} \square-\left(1-\xi^{-1}\right) \partial^{\mu} \partial_{\gamma}\right]_{x} \rho_{D}^{\gamma v}(x, y) } & =\int_{y^{0}}^{x^{0}} \mathrm{~d} z \Pi_{(\rho)}^{\mu \gamma}(x, z) \rho_{D, \gamma}{ }^{v}(z, y), \\
{\left[g^{\mu} \square-\left(1-\xi^{-1}\right) \partial^{\mu} \partial_{\gamma}\right]_{x} F_{D}^{\gamma v}(x, y) } & =\int_{t_{0}}^{x^{0}} \mathrm{~d} z \Pi_{(\rho)}^{\mu \gamma}(x, z) F_{D, \gamma}{ }^{v}(z, y) \\
& -\int_{t_{0}}^{y^{0}} \mathrm{~d} z \Pi_{(F)}^{\mu \gamma}(x, z) \rho_{D, \gamma}{ }^{v}(z, y),
\end{aligned}
$$

where we use the abbreviated notation $\int_{t_{1}}^{t_{2}} \mathrm{~d} z \equiv \int_{t_{1}}^{t_{2}} \mathrm{~d} z^{0} \int_{-\infty}^{\infty} \mathrm{d}^{d} z$. Here the initial time is denoted by $t_{0}$, which was taken without loss of generality to be zero in the respective

46 This can also be directly verified from (377) to the given order of approximation. 
equations (138) for scalars. The equations of motion for the fermion spectral and statistical correlators are obtained in a similar way from (141) as described in Sec. 3.4.3 and are given in Eq. (142).

A similar discussion as for the two-point functions can also be done for the higher correlation functions. For the three-vertex we write

$$
V^{\mu}(x, y ; z)=V_{0}^{\mu}(x, y ; z)+\bar{V}^{\mu}(x, y ; z) .
$$

and the corresponding decomposition into spectral and statistical components reads

$$
\begin{aligned}
& \bar{V}^{\mu}(x, y ; z)= \\
& U_{(F)}^{\mu}(x, y ; z) \operatorname{sign}\left(y^{0}-x^{0}\right) \operatorname{sign}\left(z^{0}-x^{0}\right)-\frac{i}{2} U_{(\rho)}^{\mu}(x, y ; z) \operatorname{sign}\left(y^{0}-z^{0}\right) \\
& +V_{(F)}^{\mu}(x, y ; z) \operatorname{sign}\left(x^{0}-z^{0}\right) \operatorname{sign}\left(y^{0}-z^{0}\right)-\frac{i}{2} V_{(\rho)}^{\mu}(x, y ; z) \operatorname{sign}\left(x^{0}-y^{0}\right) \\
& +W_{(F)}^{\mu}(x, y ; z) \operatorname{sign}\left(z^{0}-y^{0}\right) \operatorname{sign}\left(x^{0}-y^{0}\right)-\frac{i}{2} W_{(\rho)}^{\mu}(x, y ; z) \operatorname{sign}\left(z^{0}-x^{0}\right) .
\end{aligned}
$$

To discuss this in more detail we use the short-hand notation

$$
\Theta\left(x^{0}, y^{0}, z^{0}\right) \equiv \Theta\left(x^{0}-y^{0}\right) \Theta\left(y^{0}-z^{0}\right) .
$$

With the separation of Eq. (392), the time-ordered three-vertex can be written as (cf. also the corresponding discussion for two-point functions in Sec. 3.4.1)

$$
\begin{aligned}
\bar{V}^{\mu}(x, y ; z) & =V_{(1)}^{\mu}(x, y ; z) \Theta\left(x^{0}, y^{0}, z^{0}\right)+V_{(2)}^{\mu}(x, y ; z) \Theta\left(y^{0}, z^{0}, x^{0}\right) \\
& +V_{(3)}^{\mu}(x, y ; z) \Theta\left(z^{0}, x^{0}, y^{0}\right)+V_{(4)}^{\mu}(x, y ; z) \Theta\left(z^{0}, y^{0}, x^{0}\right) \\
& +V_{(5)}^{\mu}(x, y ; z) \Theta\left(x^{0}, z^{0}, y^{0}\right)+V_{(6)}^{\mu}(x, y ; z) \Theta\left(y^{0}, x^{0}, z^{0}\right),
\end{aligned}
$$

with 'coefficients' $V_{(i)}^{\mu}(x, y ; z), i=1, \ldots, 6$. These coefficients can be expressed in terms of three spectral vertex functions $U_{(\rho)}^{\mu}(x, y ; z), V_{(\rho)}^{\mu}(x, y ; z)$ and $W_{(\rho)}^{\mu}(x, y ; z)$, as well as the corresponding statistical components $U_{(F)}^{\mu}(x, y ; z), V_{(F)}^{\mu}(x, y ; z)$ and $W_{(F)}^{\mu}(x, y ; z)$ that have been employed in Eq. (393). One finds, suppressing the space-time arguments:

$$
\begin{aligned}
V_{(1)}^{\mu} & \equiv U_{(F)}^{\mu}+V_{(F)}^{\mu}-W_{(F)}^{\mu}-\frac{i}{2}\left(U_{(\rho)}^{\mu}+V_{(\rho)}^{\mu}-W_{(\rho)}^{\mu}\right), \\
V_{(2)}^{\mu} & \equiv U_{(F)}^{\mu}-V_{(F)}^{\mu}+W_{(F)}^{\mu}-\frac{i}{2}\left(U_{(\rho)}^{\mu}-V_{(\rho)}^{\mu}+W_{(\rho)}^{\mu}\right), \\
V_{(3)}^{\mu} & \equiv-U_{(F)}^{\mu}+V_{(F)}^{\mu}+W_{(F)}^{\mu}-\frac{i}{2}\left(-U_{(\rho)}^{\mu}+V_{(\rho)}^{\mu}+W_{(\rho)}^{\mu}\right), \\
V_{(4)}^{\mu} & \equiv U_{(F)}^{\mu}+V_{(F)}^{\mu}-W_{(F)}^{\mu}+\frac{i}{2}\left(U_{(\rho)}^{\mu}+V_{(\rho)}^{\mu}-W_{(\rho)}^{\mu}\right), \\
V_{(5)}^{\mu} & \equiv U_{(F)}^{\mu}-V_{(F)}^{\mu}+W_{(F)}^{\mu}+\frac{i}{2}\left(U_{(\rho)}^{\mu}-V_{(\rho)}^{\mu}+W_{(\rho)}^{\mu}\right), \\
V_{(6)}^{\mu} & \equiv-U_{(F)}^{\mu}+V_{(F)}^{\mu}+W_{(F)}^{\mu}+\frac{i}{2}\left(-U_{(\rho)}^{\mu}+V_{(\rho)}^{\mu}+W_{(\rho)}^{\mu}\right) .
\end{aligned}
$$


In terms of the coefficients $V_{(i)}^{\mu}$ these are given by:

$$
\begin{array}{rlrl}
U_{(F)}^{\mu} & =\frac{1}{4}\left(V_{(1)}^{\mu}+V_{(2)}^{\mu}+V_{(4)}^{\mu}+V_{(5)}^{\mu}\right), & U_{(\rho)}^{\mu} & =\frac{i}{2}\left(V_{(1)}^{\mu}+V_{(2)}^{\mu}-V_{(4)}^{\mu}-V_{(5)}^{\mu}\right), \\
V_{(F)}^{\mu} & =\frac{1}{4}\left(V_{(1)}^{\mu}+V_{(3)}^{\mu}+V_{(4)}^{\mu}+V_{(6)}^{\mu}\right), & V_{(\rho)}^{\mu}=\frac{i}{2}\left(V_{(1)}^{\mu}+V_{(3)}^{\mu}-V_{(4)}^{\mu}-V_{(6)}^{\mu}\right), \\
W_{(F)}^{\mu}=\frac{1}{4}\left(V_{(2)}^{\mu}+V_{(3)}^{\mu}+V_{(5)}^{\mu}+V_{(6)}^{\mu}\right), & W_{(\rho)}^{\mu}=\frac{i}{2}\left(V_{(2)}^{\mu}+V_{(3)}^{\mu}-V_{(5)}^{\mu}-V_{(6)}^{\mu}\right) .
\end{array}
$$

Insertion shows the equivalence of (395) and (393).

\subsection{Kinetic theory}

To make contact with frequent discussions in the literature, we will consider for the above gauge field and fermion nonequilibrium equations a standard "on-shell" approximation which is typically employed to derive kinetic equations for effective particle number densities. This part can be viewed as a continuation of Sec. 4.1.3, where scalar fields and the limitations of "on-shell" approximations have been discussed.

\subsection{1. "On-shell" approximations}

The evolution equations (390)-(142) to order $g^{2}$ and higher contain "off-shell" and "memory" effects due to their time integrals on the r.h.s. (cf. also Sec. 4.1). To simplify the description one conventionally considers a number of additional assumptions which finally lead to effective kinetic or Boltzmann-type descriptions for "on-shell" particle number distributions. The derivation of kinetic equations for the two-point functions $F^{\mu v}(x, y)$ and $\rho^{\mu v}(x, y)$ of Sec. 6.2.3 can be based on (i) the restriction that the initial condition for the time evolution problem is specified in the remote past, i.e. $t_{0} \rightarrow-\infty$, (ii) a derivative expansion in the center variable $X=(x+y) / 2$, and (iii) a "quasiparticle" picture. In contrast to the discussion for scalar fields in Sec. 4.1.3, within this approach one first sends the initial time $t_{0}$ to the remote past in the equations (390)-(142). This allows one to use standard derivative expansion techniques in a straightforward way. The procedure of Sec. 4.1.3 has the advantage that one can discuss which contributions to the evolution are lost in this limit. On the other hand, the advantage of the derivative expansion is that it may in principle be used to include higher order corrections. However, the complexity of a derivative expansion grows rapidly beyond the lowest order.

For the sake of simplicity (not required), we consider the Feynman gauge $\xi=1$ in the following. We will also consider a chirally symmetric theory, i.e. no vacuum fermion mass, along with parity and $C P$ invariance. Therefore, the system is charge neutral and, in particular, the most general fermion two-point functions can be written in terms of vector components only: $F^{(f)}(x, y)=\gamma_{\mu} F^{(f) \mu}(x, y), \rho^{(f)}(x, y)=\gamma_{\mu} \rho^{(f) \mu}(x, y)$, with hermiticity properties $F^{(f) \mu}(x, y)=\left[F^{(f) \mu}(y, x)\right]^{*}, \rho^{(f) \mu}(x, y)=-\left[\rho^{(f) \mu}(y, x)\right]^{*}$. For the gauge fields the respective properties of the statistical and spectral correlators read $F_{D}^{\mu \nu}(x, y)=\left[F_{D}^{v \mu}(y, x)\right]^{*}, \rho_{D}^{\mu v}(x, y)=-\left[\rho_{D}^{v \mu}(y, x)\right]^{*}$. 
In order to Fourier transform with respect to the relative coordinate $s^{\mu}=x^{\mu}-y^{\mu}$, we write

$$
\begin{aligned}
& \tilde{F}_{D}^{\mu v}(X, k)=\int \mathrm{d}^{4} s e^{i k s} F_{D}^{\mu v}\left(X+\frac{s}{2}, X-\frac{s}{2}\right), \\
& \tilde{\rho}_{D}^{\mu v}(X, k)=-i \int \mathrm{d}^{4} s e^{i k s} \rho_{D}^{\mu v}\left(X+\frac{s}{2}, X-\frac{s}{2}\right),
\end{aligned}
$$

and equivalently for the fermion statistical and spectral function, $\tilde{F}^{(f)}(X, k)$ and $\tilde{\rho}^{(f)}(X, k)$. Here we have introduced a factor $-i$ in the definition of the spectral function transform for convenience. For the Fourier transformed quantities we note the following hermiticity properties, for the gauge fields: $\left[\tilde{F}_{D}^{\mu v}(X, k)\right]^{*}=$ $\tilde{F}_{D}^{v \mu}(X, k),\left[\tilde{\rho}_{D}^{\mu v}(X, k)\right]^{*}=\tilde{\rho}_{D}^{v \mu}(X, k)$, and for the vector components of the fermion fields: $\left[\tilde{F}^{(f) \mu}(X, k)\right]^{*}=\tilde{F}^{(f) \mu}(X, k),\left[\tilde{\rho}^{(f) \mu}(X, k)\right]^{*}=\tilde{\rho}^{(f) \mu}(X, k)$. After sending $t_{0} \rightarrow-\infty$ the derivative expansion can be efficiently applied to the exact Eqs. (390) (142). Here one considers the difference of (390) and the one with interchanged coordinates $x$ and $y$, and equivalently for the other equations. We use

$$
\begin{aligned}
\int \mathrm{d}^{4} s e^{i k s} \int \mathrm{d}^{4} z f(x, z) g(z, y) & =\tilde{f}(X, k) \tilde{g}(X, k)+\ldots \\
\int \mathrm{d}^{4} s e^{i k s} \int \mathrm{d}^{4} z \int \mathrm{d}^{4} z^{\prime} f(x, z) g\left(z, z^{\prime}\right) h\left(z^{\prime}, y\right) & =\tilde{f}(X, k) \tilde{g}(X, k) \tilde{h}(X, k)+\ldots
\end{aligned}
$$

where the dots indicate derivative terms, which will be neglected. E.g. the first derivative corrections to (399) can be written as a Poisson bracket, which is in particular important if "finite-width" effects of the spectral function are taken into account. However, a typical quasiparticle picture which employs a free-field or "zero-width" form of the spectral function is consistent with neglecting derivative terms in the scattering part. We also note that the quasiparticle/free-field form of the two-point functions implies

$$
F_{D}^{\mu v}(X, k) \rightarrow-g^{\mu v} F_{D}(X, k) \quad, \quad \rho_{D}^{\mu v}(X, k) \rightarrow-g^{\mu v} \rho_{D}(X, k) .
$$

At this point the only use of the above replacement is that all Lorentz contractions can be done. This doesn't affect the derivative expansion but keeps the notation simple. Similar to Eq. (398), we define the Lorentz contracted self-energies:

$$
\begin{aligned}
-4 \tilde{\Pi}_{(F)}(X, k) & \equiv \int \mathrm{d}^{4} s e^{i k s} \Pi_{(F) \mu}^{\mu}\left(X+\frac{s}{2}, X-\frac{s}{2}\right) \\
-4 \tilde{\Pi}_{(\rho)}(X, k) & \equiv-i \int \mathrm{d}^{4} s e^{i k s} \Pi_{(\rho) \mu}^{\mu}\left(X+\frac{s}{2}, X-\frac{s}{2}\right) .
\end{aligned}
$$

Without further assumptions, i.e. using the above notation and applying the approximation (399) and (400) to the exact evolution equations one has ${ }^{47}$

$$
2 k^{\mu} \frac{\partial}{\partial X^{\mu}} \tilde{F}_{D}(X, k)=\tilde{\Pi}_{(\rho)}(X, k) \tilde{F}_{D}(X, k)-\tilde{\Pi}_{(F)}(X, k) \tilde{\rho}_{D}(X, k)
$$

47 The relation to a more conventional form of the equations can be seen by writing:

$$
\left(\tilde{\Pi}_{(\rho)} \tilde{F}_{D}-\tilde{\Pi}_{(F)} \tilde{\rho}_{D}\right)(X, k)=
$$




$$
2 k^{\mu} \frac{\partial}{\partial X^{\mu}} \tilde{\rho}_{D}(X, k)=0 .
$$

One observes that the equations (403) and (404) have a structure reminiscent of that for the exact equations for vanishing "background" fields, (390) and (391), evaluated at equal times $x^{0}=y^{0}$. However, one should keep in mind that (403) and (404) are, in particular, only valid for initial conditions specified in the remote past and neglecting gradients in the collision part.

From (404) one observes that in this approximation the spectral function receives no contribution from scattering described by the r.h.s. of the exact equation (390). As a consequence, the spectral function obeys the free-field equations of motion. In particular, $\rho_{D}^{\mu \nu}(x, y)$ have to fulfill the equal-time commutation relations $\left[\rho_{D}^{\mu \nu}(x, y)\right]_{x^{0}=y^{0}}=0$ and $\left[\partial_{x^{0}} \rho_{D}^{\mu v}(x, y)\right]_{x^{0}=y^{0}}=-g^{\mu v} \delta(\mathbf{x}-\mathbf{y})$ in Feynman gauge. The Wigner transformed freefield solution solving (404) then reads $\tilde{\rho}_{D}(X, k)=\tilde{\rho}_{D}(k)=2 \pi \operatorname{sign}\left(k^{0}\right) \delta\left(k^{2}\right)$. A very similar discussion can be done as well for the evolution equations (142) for fermions, which is massless due to chiral symmetry as stated above. Again, in lowest order in the derivative expansion the fermion spectral function obeys the free-field equations of motion and one has $\tilde{\rho}^{(f)}(X, k)=\tilde{\rho}^{(f)}(k)=2 \pi k \operatorname{sign}\left(k^{0}\right) \delta\left(k^{2}\right)$.

Assuming a "generalized fluctuation-dissipation relation" or so-called "KadanoffBaym ansatz":

$$
\begin{aligned}
\tilde{F}_{D}(X, k) & =\left[\frac{1}{2}+n_{D}(X, k)\right] \tilde{\rho}_{D}(X, k), \\
\tilde{F}^{(f)}(X, k) & =\left[\frac{1}{2}-n^{(f)}(X, k)\right] \tilde{\rho}^{(f)}(X, k),
\end{aligned}
$$

one may extract the kinetic equations for the effective photon and fermion particle numbers $n_{D}$ and $n^{(f)}$, respectively. Considering spatially homogeneous, isotropic systems for simplicity, we define the on-shell quasiparticle numbers $\left(t \equiv X^{0}\right)$

$$
\left.n_{D}(t, \mathbf{k}) \equiv n_{D}(t, k)\right|_{k^{0}=\mathbf{k}} \quad,\left.\quad n^{(f)}(t, \mathbf{k}) \equiv n^{(f)}(t, k)\right|_{k^{0}=\mathbf{k}}
$$

and look for the evolution equation for $n_{D}(t, \mathbf{k})$. Here it is useful to note the symmetry properties

$$
\begin{aligned}
\tilde{F}_{D}(t,-k) & =\tilde{F}_{D}(t, k), \tilde{\rho}_{D}(t,-k)=-\tilde{\rho}_{D}(t, k), \\
\tilde{F}^{(f)}(t,-k)^{\mu} & =-\tilde{F}^{(f)}(t, k)^{\mu}, \tilde{\rho}^{(f)}(t,-k)^{\mu}=\tilde{\rho}^{(f)}(t, k)^{\mu} .
\end{aligned}
$$

Applied to the quasiparticle ansatz (405) these imply

$$
\begin{aligned}
& n_{D}(t,-k)=-\left[n_{D}(t, k)+1\right], n^{(f)}(t,-k)=-\left[n^{(f)}(t, k)-1\right] . \\
& \quad\left(\left[\tilde{\Pi}_{(F)}+\frac{1}{2} \tilde{\Pi}_{(\rho)}\right]\left[\tilde{F}_{D}-\frac{1}{2} \tilde{\rho}_{D}\right]-\left[\tilde{\Pi}_{(F)}-\frac{1}{2} \tilde{\Pi}_{(\rho)}\right]\left[\tilde{F}_{D}+\frac{1}{2} \tilde{\rho}_{D}\right]\right)(X, k) .
\end{aligned}
$$

The difference of the two terms on the r.h.s. can be directly interpreted as the difference of a so-called "loss" and a "gain" term in a Boltzmann-type description. 


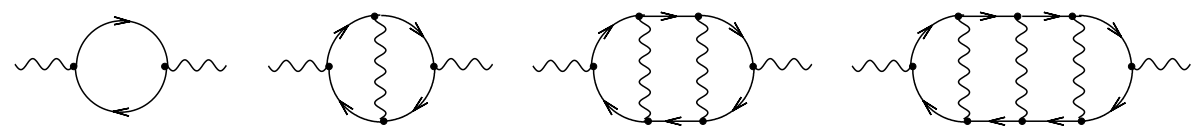

FIGURE 26. Infinite series of self-energy contributions with dressed propagator lines and classical vertices.

This is employed to rewrite terms with negative values of $k^{0}$. To order $g^{2}$ the selfenergies read (cf. Eq. (387)):

$$
\begin{aligned}
\tilde{\Pi}_{(F)}(X, k)= & 2 g^{2} \int \frac{\mathrm{d}^{4} p}{(2 \pi)^{4}}\left[\tilde{F}^{(f)}(X, k+p)^{\mu} \tilde{F}^{(f)}(X, p)_{\mu}\right. \\
& \left.-\frac{1}{4} \tilde{\rho}^{(f)}(X, k+p)^{\mu} \tilde{\rho}^{(f)}(X, p)_{\mu}\right], \\
\tilde{\Pi}_{(\rho)}(X, k)= & 2 g^{2} \int \frac{\mathrm{d}^{4} p}{(2 \pi)^{4}}\left[\tilde{F}^{(f)}(X, k+p)^{\mu} \tilde{\rho}^{(f)}(X, p)_{\mu}\right. \\
& \left.-\tilde{\rho}^{(f)}(X, k+p)^{\mu} \tilde{F}^{(f)}(X, p)_{\mu}\right] .
\end{aligned}
$$

From the equations (403) and (405) one finds at this order: $(\mathbf{q} \equiv \mathbf{k}-\mathbf{p})$

$$
\begin{aligned}
\partial_{t} n_{D}(t, \mathbf{k})=g^{2} k^{2} \int \frac{\mathrm{d}^{3} p}{(2 \pi)^{3}} \frac{1}{2 \mathbf{k} 2 \mathbf{p} 2 \mathbf{q}}\{ \\
\quad\left(n^{(f)}(t, \mathbf{p}) n^{(f)}(t, \mathbf{q})\left[n_{D}(t, \mathbf{k})+1\right]\right. \\
\left.\quad-\left[n^{(f)}(t, \mathbf{p})-1\right]\left[n^{(f)}(t, \mathbf{q})-1\right] n_{D}(t, \mathbf{k})\right) 2 \pi \delta(\mathbf{k}-\mathbf{p}-\mathbf{q}) \\
+\quad 2\left(\left[n^{(f)}(t, \mathbf{p})-1\right] n^{(f)}(t, \mathbf{q})\left[n_{D}(t, \mathbf{k})+1\right]\right. \\
\left.-n^{(f)}(t, \mathbf{p})\left[n^{(f)}(t, \mathbf{q})-1\right] n_{D}(t, \mathbf{k})\right) 2 \pi \delta(\mathbf{k}+\mathbf{p}-\mathbf{q}) \\
+\quad\left(\left[n^{(f)}(t, \mathbf{p})-1\right]\left[n^{(f)}(t, \mathbf{q})-1\right]\left[n_{D}(t, \mathbf{k})+1\right]\right. \\
\left.\left.-n^{(f)}(t, \mathbf{p}) n^{(f)}(t, \mathbf{q}) n_{D}(t, \mathbf{k})\right) 2 \pi \delta(\mathbf{k}+\mathbf{p}+\mathbf{q})\right\} .
\end{aligned}
$$

The r.h.s. shows the standard "gain term" minus "loss term" structure. E.g. for the case $k^{2}>0, k^{0}>0$ the interpretation is given by the elementary processes $e \bar{e} \rightarrow \gamma, e \rightarrow e \gamma$, $\bar{e} \rightarrow \bar{e} \gamma$ and " 0 " $\rightarrow e \bar{e} \gamma$ from which only the first one is not kinematically forbidden. From (410) one also recovers the fact that the "on-shell" evolution with $k^{2}=0$ vanishes identically at this order. A nonvanishing result is obtained if one takes into account "off-shell" corrections for a fermion line in the loop of the self-energy (409). As a consequence the first nonzero contribution to the self-energy starts at $\mathscr{O}\left(g^{4}\right)$.

Since the lowest order contribution to the kinetic equation is of $\mathscr{O}\left(g^{4}\right)$, the $3 \mathrm{PI}$ effective action provides a self-consistently complete starting point for its description. 
At this order the self-energies and vertex are given by Eqs. (380), (381) and (384). Starting from the three-vertex (384) consider for a moment the vertex resummation for the photon leg only, i.e. approximate the fermion-photon vertex by the classical vertex. As a consequence, one obtains:

$$
\begin{aligned}
V^{\mu}(x, y ; z) \simeq & \gamma^{\mu} \delta(x-z) \delta(z-y) \\
& -g^{2} \int_{x^{\prime} y^{\prime}} \gamma^{v} \Delta(x, z) V^{\mu}\left(x^{\prime}, y^{\prime} ; z\right) \Delta\left(y^{\prime}, y\right) \gamma^{\sigma} D_{\sigma v}(y, x)
\end{aligned}
$$

Using this expression for the photon self-energy (381), by iteration one observes that this resums all the ladder diagrams shown in Fig. 26. Here propagator lines correspond to self-energy resummed propagators whereas all vertices are given by the classical ones. In the context of kinetic equations, relevant for sufficiently homogeneous systems, the dominance of this sub-class of diagrams has been discussed in detail in the weak coupling limit in the literature (cf. the bibliography at the end of this section). One may decompose the contributions to the kinetic equation into $2 \leftrightarrow 2$ particle processes, such as $e \bar{e} \rightarrow \gamma \gamma$ annihilation in the context of QED, and inelastic " $1 \leftrightarrow 2$ " processes, such as the nearly collinear bremsstrahlung process. For the description of " $1 \leftrightarrow 2$ " processes, once Fourier transformed with respect to the relative coordinates, the gauge field propagator in (411) is required for space-like momenta. Furthermore, as seen from (410), the proper inclusion of nonzero contributions from $2 \leftrightarrow 2$ processes requires to go beyond the naive on-shell limit. In the context of the evolution equations (390) and (391) this can be achieved by employing the following identities:

$$
\begin{aligned}
F_{D}^{\mu v}(x, y) & =\lim _{t_{0} \rightarrow-\infty} \int_{t_{0}}^{x^{0}} \mathrm{~d} z \int_{t_{0}}^{y^{0}} \mathrm{~d} z^{\prime}\left[\rho_{D}(x, z) \Pi_{(F)}\left(z, z^{\prime}\right) \rho_{D}\left(z^{\prime}, y\right)\right]^{\mu v} \\
& =-\int_{-\infty}^{\infty} \mathrm{d} z \mathrm{~d} z^{\prime}\left[D_{R}(x, z) \Pi_{(F)}\left(z, z^{\prime}\right) D_{A}\left(z^{\prime}, y\right)\right]^{\mu v} \\
\rho_{D}^{\mu v}(x, y) & =\lim _{t_{0} \rightarrow-\infty} \int_{t_{0}}^{x^{0}} \mathrm{~d} z \int_{t_{0}}^{y^{0}} \mathrm{~d} z^{\prime}\left[\rho_{D}(x, z) \Pi_{(\rho)}\left(z, z^{\prime}\right) \rho_{D}\left(z^{\prime}, y\right)\right]^{\mu v} \\
& =-\int_{-\infty}^{\infty} \mathrm{d} z \mathrm{~d} z^{\prime}\left[D_{R}(x, z) \Pi_{(\rho)}\left(z, z^{\prime}\right) D_{A}\left(z^{\prime}, y\right)\right]^{\mu v},
\end{aligned}
$$

written in terms of the retarded and advanced propagators, $D_{R}(x, y)^{\mu \nu}=\Theta\left(x^{0}-\right.$ $\left.y^{0}\right) \rho_{D}(x, y)^{\mu \nu}$ and $D_{A}(x, y)^{\mu \nu}=-\Theta\left(y^{0}-x^{0}\right) \rho_{D}(x, y)^{\mu \nu}$, in order to have an unbounded time integration. The above identity follows from a straightforward application of the exact evolution equations and using the anti-symmetry property of the photon spectral function, $\left.\rho_{D}^{\mu v}(x, y)\right|_{x^{0}=y^{0}}=0$. We emphasize that the identity does not hold for an initial value problem where the initial time $t_{0}$ is finite. Similarly, one finds from (142) for the fermion two-point functions using $\left.\gamma^{0} \rho^{(f)}(x, y)\right|_{x^{0}=y^{0}}=i \delta(\mathbf{x}-\mathbf{y})$ :

$$
\begin{aligned}
& F^{(f)}(x, y)=-\int_{-\infty}^{\infty} \mathrm{d} z \mathrm{~d} z^{\prime} \Delta_{R}(x, z) \Sigma_{(F)}^{(f)}\left(z, z^{\prime}\right) \Delta_{A}\left(z^{\prime}, y\right), \\
& \rho^{(f)}(x, y)=-\int_{-\infty}^{\infty} \mathrm{d} z \mathrm{~d} z^{\prime} \Delta_{R}(x, z) \Sigma_{(\rho)}^{(f)}\left(z, z^{\prime}\right) \Delta_{A}\left(z^{\prime}, y\right),
\end{aligned}
$$


with $\Delta_{R}(x, y)=\Theta\left(x^{0}-y^{0}\right) \rho^{(f)}(x, y)$ and $\Delta_{A}(x, y)=-\Theta\left(y^{0}-x^{0}\right) \rho^{(f)}(x, y)$. Neglecting all derivative terms, i.e. using (399), and the above notation these give: ${ }^{48}$

$$
\begin{aligned}
& \tilde{F}_{D}(X, k) \simeq \tilde{D}_{R}(X, k) \tilde{\Pi}_{(F)}(X, k) \tilde{D}_{A}(X, k), \\
& \tilde{\rho}_{D}(X, k) \simeq \tilde{D}_{R}(X, k) \tilde{\Pi}_{(\rho)}(X, k) \tilde{D}_{A}(X, k),
\end{aligned}
$$

and equivalently for the fermion two-point functions. Applied to one fermion line in the one-loop contribution of Fig. 26, it is straightforward to recover the standard Boltzmann equation for $2 \leftrightarrow 2$ processes, using the $\mathscr{O}\left(g^{2}\right)$ fermion self-energies:

$$
\begin{aligned}
\tilde{\Sigma}_{(F)}(X, k)^{\mu}= & -2 g^{2} \int \frac{\mathrm{d}^{4} p}{(2 \pi)^{4}}\left[\tilde{F}_{D}(X, p) \tilde{F}^{(f)}(X, k-p)^{\mu}\right. \\
& \left.+\frac{1}{4} \tilde{\rho}_{D}(X, p) \tilde{\rho}^{(f)}(X, k-p)^{\mu}\right], \\
\tilde{\Sigma}_{(\rho)}(X, k)^{\mu}= & -2 g^{2} \int \frac{\mathrm{d}^{4} p}{(2 \pi)^{4}}\left[\tilde{F}_{D}(X, p) \tilde{\rho}^{(f)}(X, k-p)^{\mu}\right. \\
& \left.+\tilde{\rho}_{D}(X, p) \tilde{F}^{(f)}(X, k-p)^{\mu}\right] .
\end{aligned}
$$

For the Boltzmann equation $\Delta_{R}$ and $\Delta_{A}$ are taken to enter the scattering matrix element, which is evaluated in (e.g. "hard thermal loops" resummed) equilibrium, whereas all other lines are taken to be "on-shell". The contributions from the $1 \leftrightarrow 2$ processes may be obtained with the help of (414) with the $\mathscr{O}\left(g^{2}\right)$ photon self-energies (409). Of course, simply adding the contributions from $2 \leftrightarrow 2$ processes and $1 \leftrightarrow 2$ processes entails the problem of double counting since a diagram enters twice. This occurs whenever the internal line in a $2 \leftrightarrow 2$ process is kinematically allowed to go on-shell and has to be suppressed.

\subsubsection{Discussion}

In view of the generalized fluctuation-dissipation relation (405) employed in the above "derivation", one could be tempted to say that for consistency an equivalent relation should be valid for the self-energies as well:

$$
\tilde{\Pi}_{(F)}(X, k)=\left[\frac{1}{2}+n_{D}(X, k)\right] \tilde{\Pi}_{(\rho)}(X, k) .
$$

Such a relation is indeed valid in thermal equilibrium, where all dependence on the center coordinate $X$ is lost. Furthermore, the above relation can be shown to be a consequence of (405) using the identities (412) in a lowest-order derivative expansion: Together with Eq. (414) the above relation for the self-energies is a direct consequence

\footnotetext{
48 As for the spectral function $\rho(X, k)$ in Eq. (398), the Fourier transform of the retarded and advanced propagators includes a factor of $-i$.
} 
of the ansatz (405). However, clearly this is too strong a constraint since the evolution equation (403) would become trivial in this case: Eq. (405) and (416) lead to a vanishing r.h.s. of the evolution equation for $\tilde{F}_{D}(X, k)$ and there would be no evolution.

The above argument is just a manifestation of the well-known fact that the kinetic equation is not a self-consistent approximation to the dynamics. The discussion of Sec. 6.3.1 takes into account the effect of scattering for the dynamics of effective occupation numbers, while keeping the spectrum free-field theory like. In contrast, the same scattering does induce a finite width for the spectral function in the self-consistent $n$ PI approximation discussed in Sec. 6.2.3 because of a nonvanishing imaginary part of the self-energy (cf. also the discussion and explicit solution of a similar Yukawa model in Sec. 4.2 and the discussion in Sec. 4.1).

Though particle number is not well-defined in an interacting relativistic quantum field theory in the absence of conserved charges, the concept of time-evolving effective particle numbers in an interacting theory is useful in the presence of a clear separation of scales. Much progress has been achieved in the quantitative understanding of kinetic descriptions in the vicinity of thermal equilibrium for gauge theories at high temperature, which is well documented in the literature and for further reading we refer to the bibliography below.

For gauge theories the employed on-shell limit circumvents problems of gauge invariance or subtle aspects of renormalization. We emphasize that renormalization for linear symmetries as realized in QED can be treated along similar lines as discussed in Sec. 2.2. The generalization to nonabelian gauge theories is, however, technically more involved and needs to be further investigated.

A derivative expansion is typically not valid at early times where the time evolution can exhibit a strong dependence on $X$, and the homogeneity requirement underlying kinetic descriptions may only be fulfilled at sufficiently late times. This has been extensively discussed in the context of scalar and fermionic theories in Sec. 4. Homogeneity is certainly realized at late times sufficiently close to the thermal limit, since for thermal equilibrium the correlators do strictly not depend on $X$. Of course, by construction kinetic equations are not meant to discuss the detailed early-time behavior since the initial time $t_{0}$ is sent to the remote past. For practical purposes, in this context one typically specifies the initial condition for the effective particle number distribution at some finite time and approximates the evolution by the equations with $t_{0} \rightarrow-\infty$. The role of finite-time effects has been controversially discussed in the recent literature in the context of photon production in relativistic plasmas at high temperature. Here a solution of the proper initial-time equations as discussed in Sec. 6.2.3 seems mandatory.

\section{ACKNOWLEDGEMENTS}

I thank Gert Aarts, Daria Ahrensmeier, Rudolf Baier, Szabolcs Borsányi, Jürgen Cox, Markus M. Müller, Urko Reinosa, Julien Serreau and Christof Wetterich for very fruitful collaborations on this topic. 


\section{BIBLIOGRAPHICAL NOTES}

I apologize for the omission of many interesting contributions to this wide topic in the annotated list below, which concentrates on relativistic quantum field theory applications related to the content of the text presented above.

- A rather recent short review with a more comprehensive list of references can be found in: Progress in nonequilibrium quantum field theory, J. Berges and J. Serreau, in Strong and Electroweak Matter 2002, ed. M.G. Schmidt (World Scientific, 2003), http://arXiv:hep-ph/0302210.

- General discussions on $n \mathrm{PI}$ effective actions include: Effective Action For Composite Operators, J. M. Cornwall, R. Jackiw and E. Tomboulis, Phys. Rev. D 10 (1974) 2428. Higher effective actions for bose systems, H. Kleinert, Fortschritte der Physik 30 (1982) 187. Functional Methods in Quantum Field Theory and Statistical Physics, A.N. Vasiliev, Gordon and Breach Science Pub. (1998). nPI effective action techniques for gauge theories, J. Berges, Phys. Rev. D in print, http://arXiv:hep-ph/0401172. Gauge-fixing dependence of Phi-derivable approximations, A. Arrizabalaga and J. Smit, Phys. Rev. D 66 (2002) 065014.

- The nonperturbative 2PI 1/N expansion is derived in: Controlled nonperturbative dynamics of quantum fields out of equilibrium, J. Berges, Nucl. Phys. A699 (2002) 847; Far-from-equilibrium dynamics with broken symmetries from the $1 / \mathrm{N}$ expansion of the 2PI effective action, G. Aarts, D. Ahrensmeier, R. Baier, J. Berges and J. Serreau, Phys. Rev. D66 (2002) 045008. The latter discusses also the relation to the similar approximation scheme of Resumming the large- $N$ approximation for time evolving quantum systems, B. Mihaila, F. Cooper and J. F. Dawson, Phys. Rev. D 63 (2001) 096003

- Far-from-equilibrium quantum fields and thermalisation are discussed in: Thermalization of Quantum Fields from Time-Reversal Invariant Evolution Equations, J. Berges and J. Cox, Phys. Lett. B517 (2001) 369-374. Nonequilibrium time evolution of the spectral function in quantum field theory, G. Aarts and J. Berges, Phys. Rev. D 64 (2001) 105010. Controlled nonperturbative dynamics of quantum fields out of equilibrium, J. Berges, Nucl. Phys. A699 (2002) 847. Quantum dynamics of phase transitions in broken symmetry $\phi^{4}$ field theory, F. Cooper, J. F. Dawson and B. Mihaila, Phys. Rev. D67 (2003) 056003. Thermalization of fermionic quantum fields, J. Berges, Sz. Borsányi and J. Serreau, Nucl. Phys. B660 (2003) 52. BoseEinstein condensation without chemical potential, D. J. Bedingham, Phys. Rev. D 68 (2003) 105007. Quantum dynamics and thermalization for out-of-equilibrium phi**4-theory, S. Juchem, W. Cassing and C. Greiner, Phys. Rev. D 69 (2004) 025006.

- The phenomenon of prethermalization is discussed in: Prethermalization, J. Berges, Sz. Borsányi and C. Wetterich, Phys. Rev. Lett. in print, http://arXiv:hep$\mathrm{ph} / 0403234$

- Far-from-equilibrium dynamics of macroscopic fields with large fluctuations using the $2 \mathrm{PI} 1 / N$ expansion are discussed in: Parametric resonance in quantum field theory, J. Berges and J. Serreau, Phys. Rev. Lett. 91 (2003) 111601. The leading-order large- $N$ description has been given in: Analytic and numerical study of preheating 
dynamics, D. Boyanovsky, H. J. de Vega, R. Holman and J. F. Salgado, Phys. Rev. D 54 (1996) 7570.

- A detailed discussion about Gaussian initial density matrices and dynamics can be found in: Nonequilibrium dynamics of symmetry breaking in lambda Phi**4 field, F. Cooper, S. Habib, Y. Kluger and E. Mottola, Phys. Rev. D 55 (1997) 6471. Gaussian dynamics for inhomogeneous fields are discussed in: Particle production and effective thermalization in inhomogeneous mean field theory, G. Aarts and J. Smit, Phys. Rev. D 61 (2000) 025002. Staying thermal with Hartree ensemble approximations, M. Salle, J. Smit and J. C. Vink, Nucl. Phys. B 625 (2002) 495. Dynamical behavior of spatially inhomogeneous relativistic lambda phi**4 quantum field theory in the Hartree approximation, L. M. Bettencourt, K. Pao and J. G. Sanderson, Phys. Rev. D 65 (2002) 025015. For a leading-order study including fermions see: Nonequilibrium dynamics of fermions in a spatially homogeneous scalar background field, J. Baacke, K. Heitmann and C. Pätzold, Phys. Rev. D 58 (1998) 125013.

- The renormalization of 2PI approximation schemes is discussed in: Renormalization in self-consistent approximations schemes at finite temperature. I: Theory, H. van Hees and J. Knoll, Phys. Rev. D 65 (2002) 025010. Renormalization of self-consistent approximation schemes II: Applications to the sunset diagram, H. Van Hees and J. Knoll, Phys. Rev. D 65 (2002) 105005. Renormalization in self-consistent approximation schemes at finite temperature III: Global symmetries, H. van Hees and J. Knoll, Phys. Rev. D 66 (2002) 025028. Renormalizability of Phi-derivable approximations in scalar phi**4 theory, J. P. Blaizot, E. Iancu and U. Reinosa, Phys. Lett. B 568 (2003) 160. Renormalization of $\Phi$-derivable approximation schemes in scalar field theories, J.-P. Blaizot, E. Iancu and U. Reinosa, Nucl. Phys. A 736 (2004) 149. Renormalizing the Schwinger-Dyson equations in the auxiliary field formulation of lambda phi**4 field theory, F. Cooper, B. Mihaila and J. F. Dawson, http://arXiv:hep-ph/0407119. Renormalized thermodynamics from the 2PI effective action, J. Berges, Sz. Borsanyi, U. Reinosa and J. Serreau, http://arXiv:hep-ph/0409123.

- Classical aspects of nonequilibrium quantum fields and precision tests of the 2PI $1 / N$ expansion are examined in: Classical aspects of quantum fields far from equilibrium, G. Aarts and J. Berges, Phys. Rev. Lett. 88 (2002) 0416039. Nonequilibrium quantum fields and the classical field theory limit, J. Berges, Nucl. Phys. A 702 (2002) 351. Tachyonic preheating using 2PI - 1/N dynamics and the classical approximation, A. Arrizabalaga, J. Smit and A. Tranberg, http://arXiv:hep$\mathrm{ph} / 0409177$.

- Classical statistical field theory studies related to approximation schemes in QFT can be found in: Exact and truncated dynamics in nonequilibrium field theory, G. Aarts, G. F. Bonini and C. Wetterich, Phys. Rev. D 63 (2001) 025012. On thermalization in classical scalar field theory, G. Aarts, G. F. Bonini, C. Wetterich, Nucl. Phys. B587 (2000) 403. Time evolution of correlation functions and thermalization, G. F. Bonini and C. Wetterich, Phys. Rev. D 60 (1999) 105026. Classical limit of time-dependent quantum field theory: A Schwinger-Dyson approach, F. Cooper, A. Khare and H. Rose, Phys. Lett. B 515 (2001) 463. K. Blagoev, F. Cooper, J. Dawson and B. Mihaila, Phys. Rev. D 64 (2001) 125003. 
For diagrammatics in classical field theory see: Finiteness of hot classical scalar field theory and the plasmon damping rate, G. Aarts and J. Smit, Phys. Lett. B 393 (1997) 395. Classical statistical mechanics and Landau damping, W. Buchmüller and A. Jakovác, Phys. Lett. B 407 (1997) 39. Classical approximation for time-dependent quantum field theory: Diagrammatic analysis for hot scalar fields, G. Aarts and J. Smit, Nucl. Phys. B511 (1998) 451.

- The relation to kinetic equations is discussed in: Nonequilibrium Quantum Fields: Closed Time Path Effective Action, Wigner Function And Boltzmann Equation, E. Calzetta and B. L. Hu, Phys. Rev. D 37 (1988) 2878; Exact Conservation Laws of the Gradient Expanded Kadanoff-Baym Equations, J. Knoll, Y. B. Ivanov and D. N. Voskresensky, Annals Phys. 293 (2001) 126. Nonequilibrium quantum fields with large fluctuations, J. Berges and M. M. Muller, in Progress in Nonequilibrium Green's Functions II, Eds. M. Bonitz and D. Semkat, World Scientific (2003) [http://arXiv:hep-ph/0209026]. Cf. also Stochastic dynamics of correlations in quantum field theory: From Schwinger-Dyson to Boltzmann-Langevin equation, E. Calzetta and B. L. Hu, Phys. Rev. D 61 (2000) 025012.

- For a discussion of transport coefficients employing 2PI effective actions see: $H y$ drodynamic transport functions from quantum kinetic field theory, E. A. Calzetta, B. L. Hu and S. A. Ramsey, Phys. Rev. D 61 (2000) 125013. Transport coefficients from the 2PI effective action, G. Aarts and J. M. Martinez Resco, Phys. Rev. D 68 (2003) 085009. Shear viscosity in the $O(N)$ model, G. Aarts and J. M. Martinez Resco, JHEP 0402 (2004) 061. Transport coefficients at leading order: Kinetic theory versus diagrams, G. D. Moore, in Strong and Electroweak Matter 2002, ed. M.G. Schmidt (World Scientific, 2003), http://arXiv:hep-ph/0211281. Cf. also Transport coefficients in high temperature gauge theories II: Beyond leading log, P. Arnold, G. D. Moore and L. G. Yaffe, JHEP 0305 (2003) 051.

- For an application to photon production rates in a non-equilibrium medium see: Out-of-equilibrium electromagnetic radiation, J. Serreau, JHEP 0405 (2004) 078.

Figures. Figs. 3-5: J. Berges, Nucl. Phys. A699 (2002) 847. Fig. 6 left: J. Berges and J. Cox, Phys. Lett. B517 (2001) 369-374. Fig. 6 right: G. Aarts and J. Berges, Phys. Rev. D 64 (2001) 105010. Figs. 7-10: J. Berges, Sz. Borsányi and C. Wetterich, Phys. Rev. Lett. in print, http://arXiv:hep-ph/0403234. Figs. 11-14: J. Berges and J. Serreau, Phys. Rev. Lett. 91 (2003) 111601. Figs. 16-18: G. Aarts and J. Berges, Phys. Rev. Lett. 88 (2002) 0416039. 